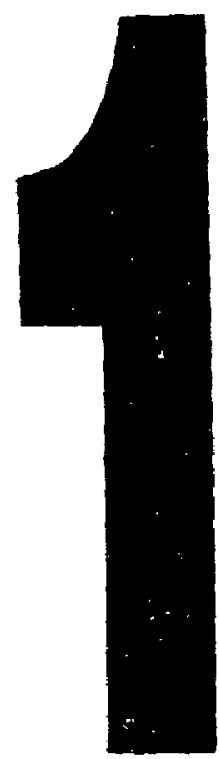

PM-1 31/2"X4" PHOTOGRAPHIC MICROCOPY TARGET NBS 1010a ANSI/ISO \#2 EQUIVALENT

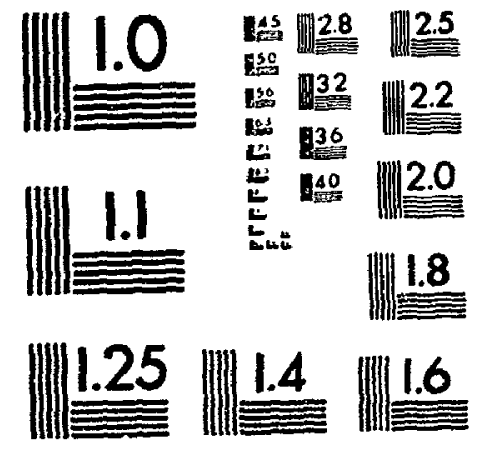

PRECISIONSM RESOLUTION TARGETS 
The quality of this microform is heavily dependent upon the quality of the original thesis submitted for microfilming. Every effort has been made to ensure the highest quality of reproduction possible.

If pages are missing, contact the university which granted the degree.

Some pages may have indistinct print especially if the original pages were typed with a poor typewriter ribbon or if the university sent us an inferior photocopy.

Reproduction in full or in part of this microform is governed by the Canadian Copyright Act, R.S.C. 1970, c. C-30, and subsequent amendments.
La qualité de cette microforme dépend grandement de la qualité de la thèse soumise au microfilmage. Nous avons tout fait pour assurer une qualité supérieure de reproduction.

S'il manque des pages, veuillez communiquer avec l'université qui a conféré le grade.

La qualité d'impression de certaines pages peut laisser à désirer, surtout si les pages originales ont été dactylographiées à l'aide d'un ruban usé ou si l'université nous a fait parvenir une photocopie de qualité inférieure.

La reproduction, même partielle, de cette microforme est soumise à la Loi canadienne sur le droit d'auteur, SRC 1970, c. C-30, et ses amendements subséquents. 


\title{
An Interpolated Frequency-Hopping Spread-Spectrum Transceiver System
}

\author{
by \\ Norman M. Filiol, B.Sc. (Eng.) \\ A thesis submitted to \\ the Faculty of Graduate Studies and Reasearch \\ in partial fulfillment of \\ the requirements for the degree of \\ Master of Engineering
}

Ottawa-Carleton Institute for Electrical Engineering

Department of Electronics

Faculty of Engineering

Carleton University

Ottawa, Canada

November, 1994

(C) 1994, Norman Filiol 
National Library

of Canada

Acquisitions and Bibliographic Services Branch

395 Wellington Street Otlawa, Ontario KIA ONA
Bibliotheque nationale

du Canada

Direction des acquisitions ef

des services bibliographiques

395. rua Wellington

Ottawa (Ontario)

KIAON4

Your the volre reterence

Out the Noreserorence

THE AUTHOR HAS GRANTED AN IRREVOCABLE NON-EXCLUSIVE LICENCE ALLOWING THE NATIONAL LIBRARY OF CANADA TO REPRODUCE, LOAN, DISTRIBUTE OR SELL COPIES OF HIS/HER THESIS BY ANY MEANS AND IN ANY FORM OR FORMAT, MAKING THIS THESIS AVAILABLE TO INTERESTED PERSONS.
L'AUTEUR A ACCORDE UNE LICENCE IRREVOCABLE ET NON EXCLUSIVE PERMETTANT A LA BIBLIOTHEQUE NATIONALE DU CANADA DE REPRODUIRE, PRETER, DISTRIBUER OU VENDRE DES COPIES DE SA THESE DE QUELQUE MANIERE ET SOUS QUELQUE FORME QUE CE SOIT POUR METTRE DES EXEM:PLAIRES DE CETTE THESE A LA DISPOSITION DES PERSONNE INTERESSEES.
THE AUTHOR RETAINS OWNERSHIP OF THE COPYRIGHT IN HIS/HER THESIS. NEITHER THE THESIS NOR SUBSTANTIAL EXTRACTS FROM IT MAY BE PRINTED OR OTHERWISE REPRODUCED WITHOUT HIS/HER PERMISSION.
L'AUTEUR CONSERVE LA PROPRIETE DU DROIT D'AUTEUR QUI PROTEGE SA THESE. NI LA THESE NI DES EXTRAITS SUBSTANTIELS DE CELLECI NE DOIVENT ETRE IMPRIMES OU AUTREMENT REPRODUITS SANS SON AUTORISATION. 
Name Plar on Maurice Filio

Dissertation Abstracts International is arranged by broad, general subject categories. Please select the one subject which most nearly describes the content of your dissertation. Enter the corresponding four-digit code in the spaces provided.

ELECTRONISS ERJSINFEFING SUBJECT TERM
0.51444 U.M.I SIJBIECT CODE

\section{Subject Categories}

\section{THE HUMANITIES AND SOCLAL SCIENCES}

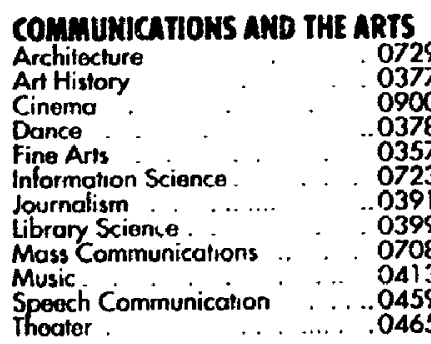

\section{EDUCATION}

Gonatral . . .. . . 0515

Administralion ..... . 0514

Agricultural $\quad \ldots . . . \ldots 517$

$\begin{array}{lll}\text { Ant. } & .0273 \\ \text { Biingual and Multicultural . . . . } 0282 \\ \text { Business }\end{array}$

Curricylum and Instruction $\quad \ldots .0727$

Eorly Childhood ... ...0518

Elemeritary . .... . ..0524

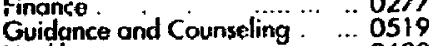

$\begin{array}{llll}\text { Hissoly of } & . & . & 0520 \\ \text { Hiome Economics } & \ldots . & . & 0278\end{array}$

Industral. .. $\quad . . . .0521$

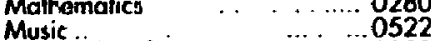

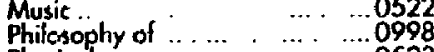

Physical. . . ...............

Slanc and East European .... 0314
Community College : . ......0685

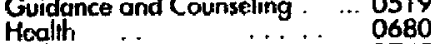

Meallh $\cdots$
Higher

Language and Literalure .........0278

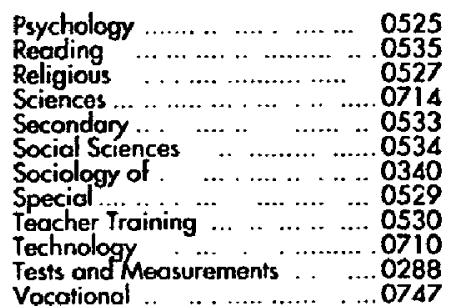

\section{LHGUAGE, LTERATURE ANB}

Language

General . ................... . .0679

Ancient $\quad \cdots \ldots \ldots . . . . . .0289$

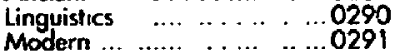

Literature

General ...................0401

Classisal ...................... 0294

Comporafive .............. . 0295

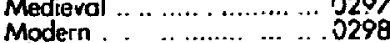

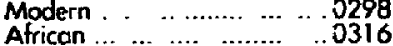

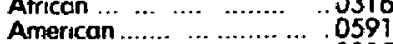

Asian ..................... 0305

Conudian (French)

English ...................... 0593

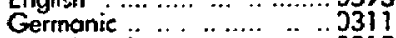

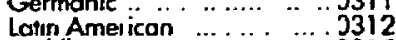

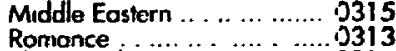

INGUSTICS

\section{PHILOSOPHY, RELIGION AHD}

THEOLOGY

Philosophy ........................0422

Religion

General ................0318

Biblical Siudies .....................0321

Clergy .......................0319

Philosophy of ................. 0322

Theology ............................... 0469

\section{SOCIAL SCIENCES}

American Sludies $\quad . . \ldots \ldots . . . . .0323$

Anthropology

Archaeology ... . . ... . . . 0324

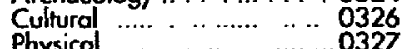

Business Administration

General ...................0310

Accounting …............. 0272

Banking ........................ 0770

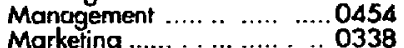

Canadion Studies ................ 0385

Economics

Generol . ......... 0501

Commerce-Business .......... 0505

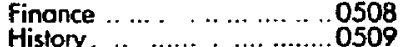

Theory. ................................. 051

Folklore ……...............................

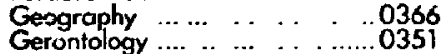

History

General

0578
Agricultural. .............0503

iobor ........................... 0510

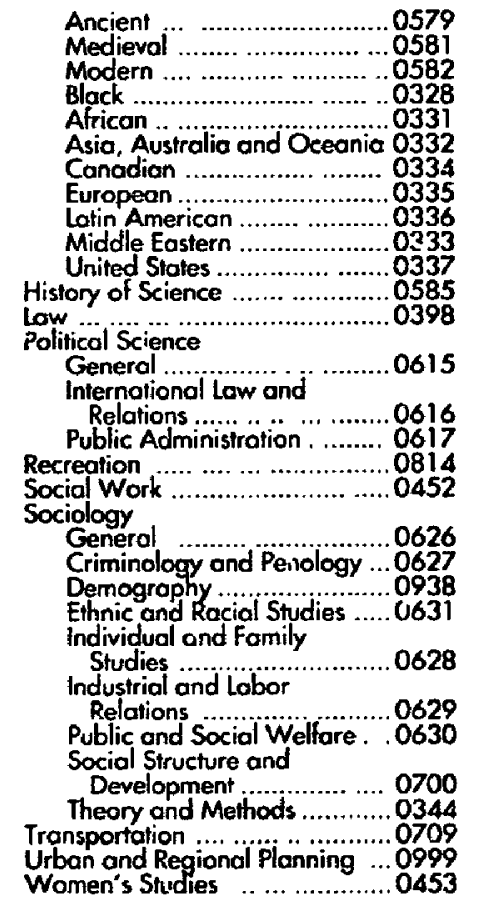

\section{THE SCIENCES AND ENBINEERINC}

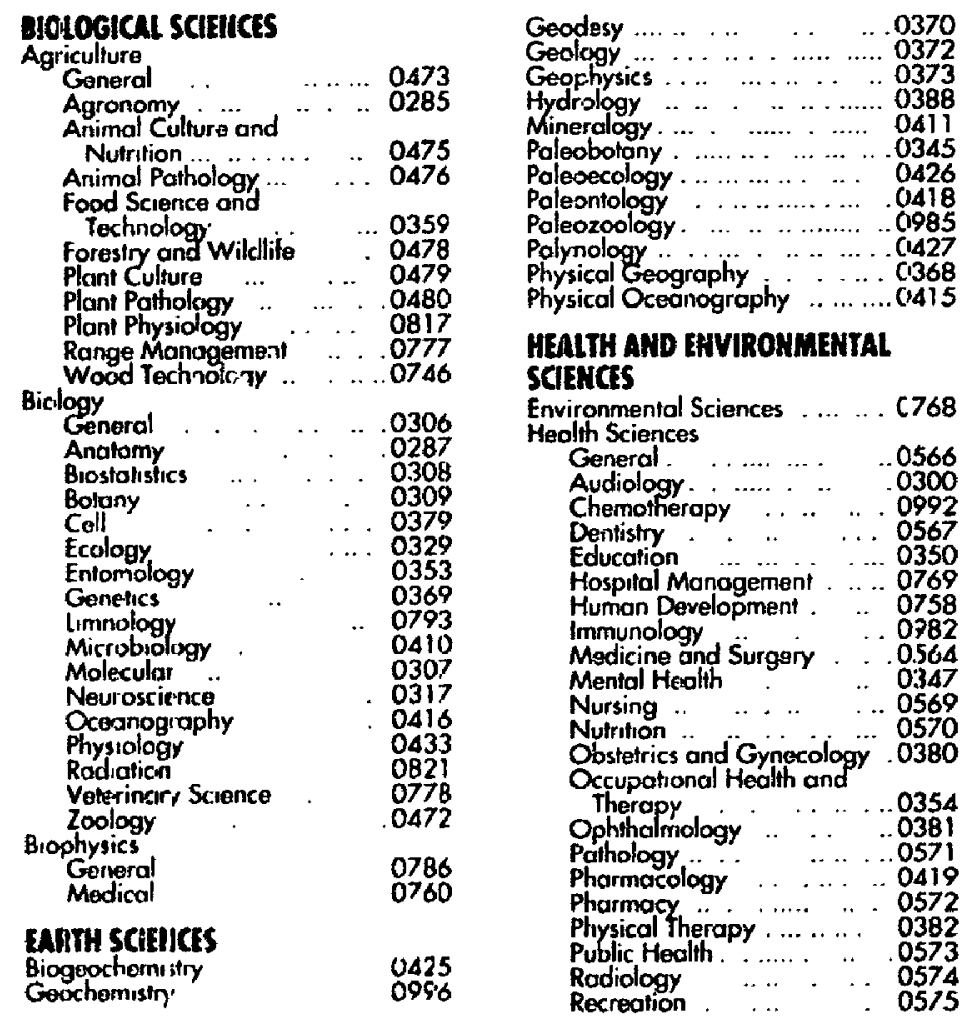

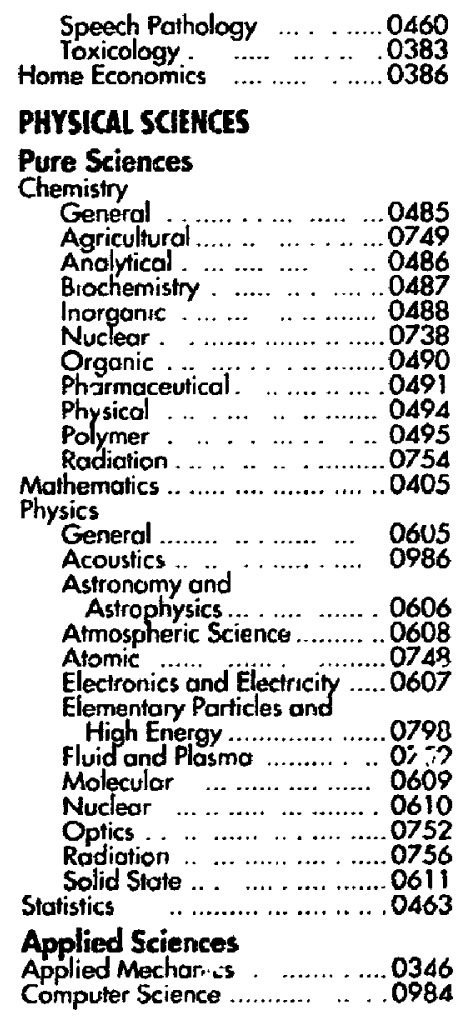

Engineering

(n..........0537

Aerospace ...................0538

Agricultural . ................. 0539

Automotive ….....................0540

Biomedical ...........................0541

Chemical ..........................0542

Electronies ond Electrical ........0544

Heat and Thermodynamics ...0348

Hydraulic ... . . . . ........ 0545

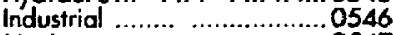

Marine ....................... $0 \$ 47$

Materials Science . . ..........0794

Mechonical . . .... . ........... 0548

Metallurgy ….................... 0743

Mining. ….................. 0555

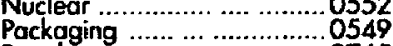

Petroleum ...................0765

Sanitary and Municipal ........ 0554
System Science. ................0790

Geofechnology 0428

Operations Research ...............0796

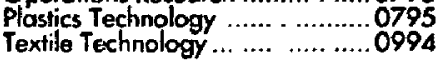

\section{PSYCHOLOGY}

General .......................0621

Behavioral ................................... 0384

(n)......0622

Developmenlal ......................0620

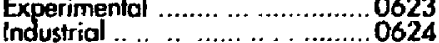

industrial

Personality.

Psychobiology

Psychometrics ......................0632

Sccial . ............................................ 
The undersigned hereby recommend to the Faculty of Graduate Studies and Research acceptance of the thesis

\title{
An Interpolated Frequency-Hopping Spread-Spectrum Transceiver System
}

\author{
submitted by
}

Norman Maurice Filiol, B.Eng.

in partial fulfilment of the requirements

for the degree of Master of Engineering.

Acting Chair, Department of Electronics

Thesis Supervisor

Ottawa-Carleton Institute for Electrical Engineering

Department of Electronics

Carleton University

Ottawa, Ontario

November 1994

(C) Copyright 


\section{Abstract}

A technique of spread-spectrum transmission, interpolated frequency-hopping (IFH), is presented which employs a carrier that moves smoothly and continuously in frequency. This helps to alleviate problems such as spectral splatter and transient mismatch, which are a concern in conventional phase-locked loop based frequency-hopping spread-spectrum systems. In interpolated frequency-hopping, the pseudo-random hopping code is passed through a digital interpolation filter prior to controlling the synthesizer instantaneous frequency output. Laboratory measurements indicate that for certain cases, interpolated frequency-hopping shows a $12.5 \mathrm{~dB}$ improvement in average received IF power, has a much sharper roll-off of inband phase noise when compared to conventional hopping, and provides a phase-coherent IF after despreading. An interpolated frequency-hopping transceiver system consisting of a $\Delta-\Sigma$ frequency synthesizer, a $\Delta-\Sigma$ frequency discriminator, and $\Delta-\Sigma$ filter tap coefficients, is proposed. The system would be suitable for integrated mobile radio applications in slow fading environments. 


\section{Acknowledgments}

I would like to extend my heartfelt thanks to my advisor Calvin Plett for his guidance throughout the course of my thesis work.

A special thanks to Terry Kenny for his initial work on broadband-coded FM, and for allowing me to look over his shoulder during the course of his thesis work.

I would like to thank Tom Riley for his help with the theoretical aspects of my thesis, and Luc Lussier for his technical advice.

I would also like to thank Peter Manashe, Mike Getz, and Jacques Lemieux of ETC electronics, as well as Nagui Mikhail for their technical support. 


\section{Table Of Contents}

1 Introduction 1

1.1 Problem Description................................................................................ 1

1.2 Motivation............................................................................................ 1

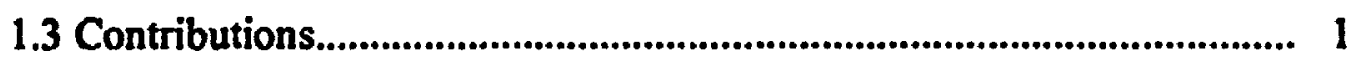

1.4 Thesis Outline.................................................................................... 2

2 Background 3

2.1 The Mobile Radio Channel.................................................................... 3

2.2 Wireless Radio Standards................................................................ 5

2.3 Trends in VLSI for Wireless Communications...................................... 7

2.4 Frequency-synthesis.................................................................................. 8

2.4.1 Direct-analog Frequency-synthesis....................................... 8

2.4.2 Direct-digital Frequency-synthesis........................................... 9

2.4.3 Indirect-analog Frequency-synthesis..................................... 10

2.5 Phase-error in Phase-locked Loops....................................................... 13

2.5.1 Phase-error Due to a Frequency Step.................................... 14

2.5.2 Response of a PLL to Random FM Modulation................... 15

2.6 Continuous-phase Modulation Techniques.......................................... 16

2.7 Spread-spectrum................................................................................. 19

2.7.1 Processing Gain................................................................ 20

2.7.2 Forms of Spread-spectrum Transmission.............................. 21

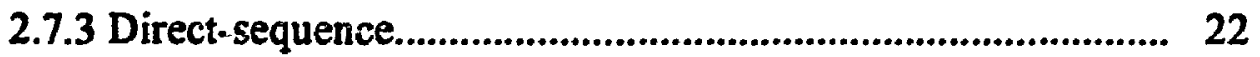

2.7.4 Frequency-hopping............................................................ 27

2.7.5 Broadband-coded FM....................................................... 32

3 Interpolated Frequency-hopping $\quad 35$

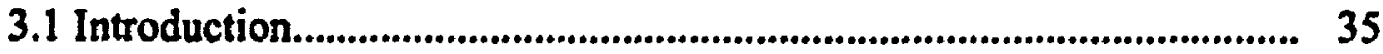

3.2 IF Distortion Due to PLL Mismatch................................................... 37

3.3 Frequency Spectrum............................................................................. 41

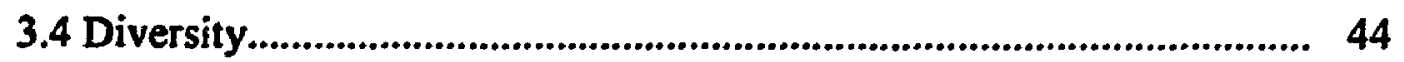


4 Proposed System 45

4.1 An Interpolated Frequency-hopping Transceiver............................... 45

5 System Design $\quad 50$

S.1 Introduction............................................................................. 50

5.2 Transmission Testbed ............................................................. 50

5.3 Hopping Code and Interpolation Filter.......................................... 53

5.4 White Noise Source and BER Board........................................... 58

5.5 Analog Frequency Discriminator.................................................. 62

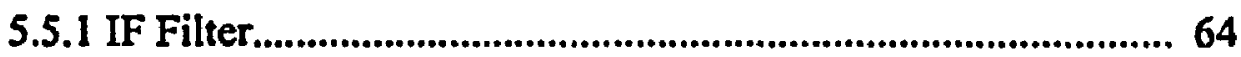

5.5.2 Phase-shift Frequency Discriminator..................................66 66

5.5.3 Passive Carrier Suppression Filter.................................... 70

5.5.4 Matched Filter and Decision Circuit...................................... 71

6 Results $\quad 73$

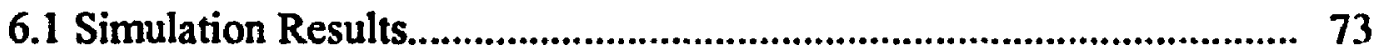

6.1.1 Simulation Methods.......................................................... 73

6.1.2 PLL Model.................................................................... 74

6.1.3 Simulation of the Carrier..................................................... 76

6.1.4 Simulation of Carrier with Data.......................................... 82

6.2 Laboratory Measurements................................................................ 84

6.2.1 Measurements Without Data................................................. 85

6.2.2 Measurements with Data................................................. 97

7 Conclusions and Future Work 113

7.1 Conclusions.................................................................... 113

7.2 Future Work...................................................................... 114

R References $\quad 116$

A BER Board Schematics $\quad$ A.1

B FSK Demodulator Board Circuit Schematics $\quad$ B.1 


\section{List of Figures}

Figure Number

Page Number

2-1 Multipath Propagation............................................................................. . 3

2-2 Direct-analog Frequency Synthesizer........................................................... 8

2-3 Direct-digital Frequency Synthesizer............................................................ 9

2-4 Phase-locked Loop Frequency Synthesizer............................................... 11

2-5 Fractional-N PL.L Based Frequency Synthesizer........................................ 12

2-6 Transient Phase-error in PLL Due to a Step in Input Frequency................. 15

2-7 Partial Response System Model................................................................... 17

2-8 Symbol Combining in a PRS System................................................ 19

2-9 Processing Gain................................................................................ 20

2-10 Direct-sequence Spread-spectrum System............................................... 23

2-11 Direct-sequence Spread-spectrum Waveforms............................................ 24

2-12 Matched filter for Direct-sequence Detection......................................... 25

2-13 Frequency-hopping Spread-spectrum System....................................... 27

2-14 Frequency-hopping Spread-spectrum.................................................... 28

2-15 PLL Transient Response to Frequency hop............................................... 30

2-16 Modulating Signal for Sinusoidal FM................................................. 33

3-1 An Interpolated Frequency-hopping Spread-spectrum System................... 36

3-2 An Interpolated Frequency-hopping Spread-spectrum Signal.................... 37

4-1 An Interpolated Frequency-hopping Spread-spectrum Transceiver............ 46

4-2 Digital Interpolation Filter................................................................. 48

5-1 Experimental Setup......................................................................... 51

5-2 Hopping Code (Frequency vs. Time)....................................................... 53

5-3 Simulated Frequency Spectrum of Hopping Code....................................... 55

5-4 Magnitude Response of Digital Interpolation Filter.................................... 56

5-5 Frequency Spectrum of Filtered Hopping Code........................................ 57

5-6 Magnitude Response of Synthesizer Mismatch Function........................... 58

5-7 White Noise Source................................................................................ 59

5-8 Real-ime Bit-error Counter................................................................. 61 
5-9 Ideal and Non-ideal FSK Receiver Structure............................................. 63

5-10 Block Diagram of FSK Demodulator Board.......................................... 63

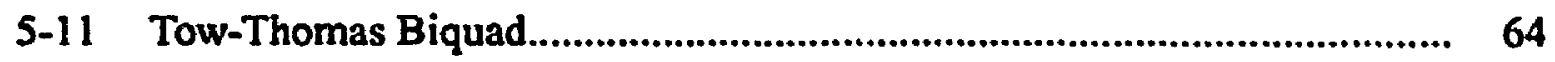

5-12 Phase-shift Frequency Discriminator.....................................................66 66

5-13 Magnitude Response Plot for LC tank Circuit......................................... 67

5-1.4 Phase Response Plot for LC Tank Circuit.................................................. 68

5-15 Passive Carrier Suppression Filter.......................................................... 71

5-16 Matched Filter and Decision Circuit.................................................... 72

5-17 Output of Integrate and Dump Filter..................................................... 72

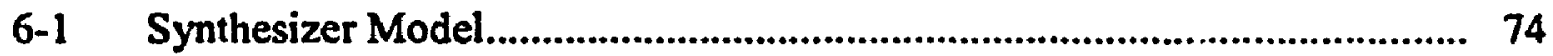

6-2 Simple and Interpolated Frequency-hopping (Frequency vs. Time)........... 77

6-3 Simple and Interpolated Frequency-hopping (Phase vs. Frequency).......... 78

6-4 Simple and Interpolated Frequency-kopping (IF Frequency-error vs. Time) 79

6-5 IF Spectrum Without Data

(Hop Rate=10 KHz, Maximum Hop size=1.25 MHz)........................... 79

6-6 IF Spectrum Without Data

(Hop rate $=10 \mathrm{KHz}$, Maximum Hop size $=2.5 \mathrm{MHz}$ )............................. 80

6-7 IF Spectrum Without Data

(Hop rate $=10 \mathrm{KHz}$, Maximum Hop size $=5.0 \mathrm{MHz}$ )............................ 80

6-8 Natural Frequency Mismatch (Rate $=10 \mathrm{KHz}$, Hop size $=1.25 \mathrm{MHz}$ )........ 81

6-9 IF Spectrum for Carrier and Binary-FSK Data......................................... 83

6-10 Baseband Spectrum for Demodulated Binary-FSK Data............................ 83

6-11 Phase Noise Measurement for Tx Synthesizer in Integer-N Operation....... 85

6-12 Phase Noise Measurement for Tx Synthesizer in Fractional-N Operation.. 86

6-13 Hopping Code (Frequency vs. time) ...................................................... 87

6-14 IF Spectrum for Stationary Carrier..................................................... 88

6-15 IF Spectrum (Hopping rate $=10 \mathrm{KHz}$, Maximum Hop size $=1.25 \mathrm{MHz}$ ).... 89

6-16 IF Spectrum (Hopping rate $=10 \mathrm{KHz}$, Maximum Hop size $=2.5 \mathrm{MHz}$ )...... 89

6-17 IF Spectrum (Hopping rate $=10 \mathrm{KHz}$, Maximum Hop size $=5.0 \mathrm{MHz}$ )...... 90

6-18 IF Spectrum (Hopping rate $=78 \mathrm{KHz}$, Maximum Hop size $=1.25 \mathrm{MHz}$ ).... 90

6-19 IF Spectrum (Hopping rate $=78 \mathrm{KHz}$, Maximum Hop size $=1.75 \mathrm{MHz}$ ).... 91

6-20 IF Spectrum (Hopping rate $=78 \mathrm{KHz}$, Maximum Hop size $=2.5 \mathrm{MHz}$ )...... 91 
6-21 IF Spectrum (Hopping rate $=78 \mathrm{KHz}$, Maximum Hop size $=5.0 \mathrm{MHz}$ )...... 92

6-22 IF Spectrum (Hopping rate $=156 \mathrm{KHz}$, Maximum Hop size $=1.25 \mathrm{MHz}$ )... 92

6-23 IF Spectrum (Hopping rate $=156 \mathrm{KHz}$, Maximum Hop size $=1.75 \mathrm{MHz}$ ).. 93

6-24 IF Spectrum (Hopping rate $=156 \mathrm{KHz}$, Maximum Hop size $=2.5 \mathrm{MHz}$ ).... 93

6-25 IF Spectrum (Hopping rate $=156 \mathrm{KHz}$, Maximum Hop size $=5.0 \mathrm{MHz}$ ).... 94

6-26 Eye Diagram for Simple Frequency-hopping

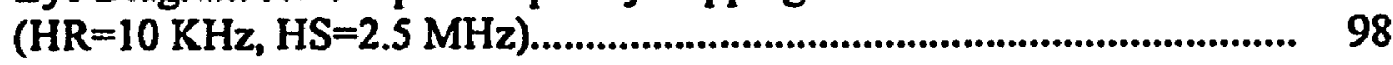

6-27 Eye Diagram for Interpolated Frequency-hopping

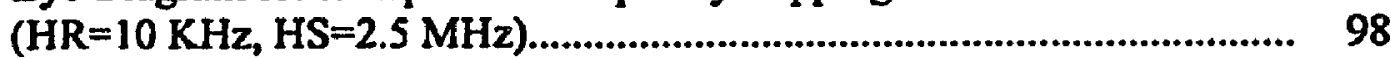

6-28 Eye Diagram for Simple Frequency-hopping

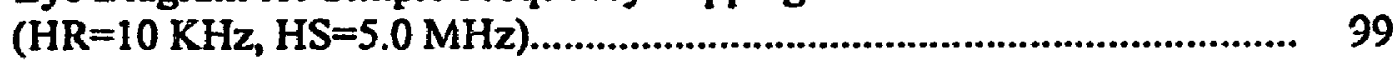

6-29 Eye Diagram for Interpolated Frequency-hopping $(\mathrm{HR}=10 \mathrm{KHz}, \mathrm{HS}=5.0 \mathrm{MHz}$ ). 99

6-30 Eye Diagram for Simple Frequency-inopping $(\mathrm{HR}=78 \mathrm{KHz}, \mathrm{HS}=2.5 \mathrm{MHz}$ ). 100

6-31 Eye Diagram for Interpolated Frequency-hopping ( $\mathrm{HR}=78 \mathrm{~K}: \mathrm{Hz}, \mathrm{HS}=2.5 \mathrm{MHz}$ ).

6-32 Eye Diagram for Simple Frequency-hopping $(\mathrm{HR}=78 \mathrm{KHz}, \mathrm{HS}=5.0 \mathrm{MHz}$.

6-33 Eye Diagram for Interpolated Frequency-hopping (HR=78 KHz, HS=5.0 MHz).

6-34 Eye Diagram for Simple Frequency-hopping $(\mathrm{HR}=156 \mathrm{KHz}, \mathrm{HS}=2.5 \mathrm{MHz}$ ).

6-35 Eye Diagram for Interpolated Frequency-hopping $(\mathrm{HR}=156 \mathrm{KHz}, \mathrm{HS}=2.5 \mathrm{MHz}$ ).

6-36 Eye Diagram for Simple Frequency-hopping $(\mathrm{HR}=156 \mathrm{KHz}, \mathrm{HS}=5.0 \mathrm{MHz}$ ).

6-37 Eye Diagram for Interpolated Frequency-hopping $(\mathrm{HR}=156 \mathrm{KHz}, \mathrm{HS}=5.0 \mathrm{MHz})$. ¿03

6-38 Bit-error Rate vs. Eb/No for Simple Frequency-hopping at $10 \mathrm{KHz} . . . . . . . . .105$ 6-39 Bit-error Rate vs. Eb/No for Interpolated Frequency-hopping at $10 \mathrm{KHz} . . .105$ 
6-40 Bit-error Rate vs. Eb/No for Simple Frequency-hopping at $78 \mathrm{KHz} \ldots \ldots \ldots . .106$

6-41 Bit-error Rate vs. Eb/No for Interpolated Frequency-hopping at 78K Hz... 106

6-42 Bit-error Rate vs. Eb/No for Simple Frequency-hopping at $156 \mathrm{KHz}$......... 107

6-43 Bit-error Rate vs. Eb/No for Interpolated Frequency-hopping at $156 \mathrm{KHz} .107$

6-44 Bit-error Rate vs. Eb/No for both the Stationary Carrier Case and The Ideal Non-Coherent FSK Case. 


\section{List Of Tables}

Table Number

Page Number

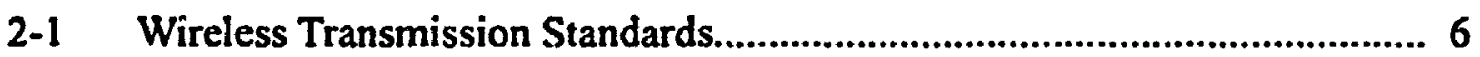

5-1 BER Board Settings............................................................................ 61

6-1 Absolute Received Power.............................................................................. 96

6-2 Bit-error Rates for Given Eb/No Values................................................... 110 


\section{List of Symbols and Abbreviations}

\begin{tabular}{ll} 
A/D & Analog-to-digital Converter \\
AM & Amplitude Modulation \\
AWGN & Additive White Gaussian Noise \\
B $_{\mathrm{d}}$ & Doppler-spread of Propagation Channel \\
BER & Bit-error Rate \\
$\beta$ & Modulation Index of FM Signal, Hopping Code \\
biCMOS & Frocess that mixes bipolar devices and CMOS devices on the same chip \\
BPF & Bandpass Filter \\
CDMA & Code-division Multiple-access \\
CMOS & Complementary Metal-oxide Semiconductor \\
CPM & Continuous-phase Modulation \\
D/A & Digital-to-analog Converter \\
DAS & Direct-analog Frequency-synthesis \\
DDS & Direct-digital Frequency-synthesis \\
$\delta$ & Delta function \\
$\Delta-\Sigma$ & Delta-sigma \\
$\Delta \omega$ & Maximum Frequency Deviation of FM Signal \\
$\Delta \omega_{i}(s)$ & Change in Input Frequency \\
DS & Direct-sequence \\
DSP & Digital Signal-processing \\
ECL & Emitter-coupled Logic \\
ENR & Excess Noise Ratio \\
FDMA & Frequency-division Multiple-access \\
FFT & Fast Fourier Transform \\
FH & Frequency-hopping \\
$f_{\mathfrak{m}}$ & Modulation Rate in Hertz \\
FM & Frequency Modulation \\
FRS & Full-response Signalling \\
FSK & Frequency-shift Keying \\
GMSK & Gaussian Minimurn-shift Keying \\
\hline
\end{tabular}




\begin{tabular}{ll}
$h_{i}(t)$ & Impulse response of ith propagation path \\
HS & Maximum Hop Size \\
HR & Hopping rate \\
IF & Intermediate Frequency \\
IFH & Interpolated Frequency-hopping \\
ISI & Inter-symbol Interference \\
ISM & Instrumentation, Scientific, and Medical Band \\
K & Loop Gain \\
L & Order of Diversity \\
LNA & Low-noise Amplifier \\
LO & Local Oscillator \\
LPF & Lowpass Filter \\
$\omega_{m}$ & Modulation Rate in Radians/s \\
PA & Power Amplifier \\
PCN & Personal Communications Network \\
PDMA & Phase-division Multiple-access \\
$\phi_{i}(t)$ & Uniformly Distributed Fhase-shift of ith Propagation Path \\
PLL & Phase-locked Loop \\
PRS & Partial-response Signalling \\
PSK & Phase-shift Keying \\
RAM & Random Access Memory \\
RBW & Resolution Bandwidth \\
RF & Radio Frequency \\
rms & Root Mean Square \\
$\rho_{\mathbf{j}}(t)$ & Rayleigh Distributed and Independent Transmittance of ith Path \\
ROM & Read-or.ly Memory \\
Rx & Receiver \\
SNR & Signal-to-noise Ratio \\
$\tau_{m}$ & Mean Delay-spread of Propagation Channel \\
TDMA & Time-division Multiple-access \\
TH & Time-hopping \\
$\theta_{\mathrm{e}}(s)$ & Phase-error \\
\hline
\end{tabular}


TTL Transistor-transistor Legic

Tx Transmitter

VBW Video Bandwidth

VCO Voltage-controlled Oscillator

VLSI Very Large Scale Integration 


\section{Chapter 1}

\section{Introduction}

\subsection{Problem Description}

Mobile radio channels present system designers with many difficult design challenges. In order to reduce these difficulties, third generation personal communication devices are using digital data transmission techniques. The use of digital transmission allows system designers to take advantage of the powerful digital signal-processing (DSP) techniques that are now available, and also improves data reception. Radio systems operating in indoor environments such as office buildings, factories, shopping malls, etc., must be robust in order to deal with the severe transmission degradation that is caused by multi-path propagation within these structures.

\subsection{Motivation}

One solution to the problems mentioned in the above paragraph is to transmit data over a wide band of frequencies. This form of transmission is called spread-spectrum. For systems that require both multiple-access capability and immunity to multi-path fading, spreadspectrum techniques may provide the only viable solution. Spread-spectrum transmission was originally used by the military, and only recently has been used in commercial applications. This transition has opened up a large area of research in mobile radio design. In fact, code-division multiple-access (CDMA) using direct-sequence spread-spectrum is now. an accepted FCC transmission standard [Mic94].

\subsection{Contributions}

This thesis builds on work performed in another thesis on a form of spread-spectrum transmission called broadband-coded FM [Ken94]. In the previous work, a broadbandcoded FM transmission system was built and tested at the hardware level. The transmit and receive frequency synthesizers used were $\mathrm{PC}$ board level fractional-N synthesizers, digitally controlled using $\Delta-\Sigma$ modulated bitstreams. The system was used to evaluate the 
quality of the received (IF) signal for sinusoidal, broadband modulation of the carrier. In this thesis, a subset of broadband-coded FM spread-spectrum transmission, interpolated frequency-hopping (IFH), is defined, simulated, and tested at the hardware level. For this purpose, a hopping code generator and digital interpolation filter were implemented in software, and used in realtime to control the synthesizers used in the previous system. In order to compare the bit-error rate performances of stationary carrier transmission, standard frequency-hopping transmission, and interpolated frequency-hopping transmission to one another, a white noise generator, FSK demodulator, and a realtime bit-error rate counter were designed, built and tested at the hardware level. It is shown by the work in this thesis that interpolated frequency-hopping transmission provides advantages over other forms of spread-spectrum, and solves many of the problems inherent in phase-locked loop (PLL) based frequency-hopping systems. Interpolated frequency-hopping is presented as an alternative form of spread-spectrum and is not meant to replace the other forms. It is loosely aimed at the instrumentation, scientific, and medical band (ISM), although no fixed transmission standard has been selected.

\subsection{Thesis Outline}

This thesis is divided into seven chapters. Chapters 1 and 2 provide a general discussion of mobile radio and spread-spectrum techniques. Chapter 3 provides a theoretical description of interpolated frequency-hopping. A proposed transceiver architecture that makes use of interpolated frequency-hopping transmission is presented in Chapter 4. Chapters 5 and 6 give details on the test system design, as well as simulation and laboratory results. Conclusions are presented in Chapter 7. 


\section{Chapter 2}

\section{Background}

\subsection{The Mobile Radio Channel}

Usually, wireless data transmission is performed using a fixed carrier frequency. However, significant portions of the transmitted data may be lost due to a channel impairment at the carrier frequency. Often, this impairment is caused by multi-path propagation of the transmitted signal. The received signal in a multi-path environment is the vector sum of all the reflected, refracted, and scattered components of the transmitted signal, as illustrated in Figure (2-1). The different components arrive at the receiver each with a random amplitude and phase, and can add constructively or destructively. If the addition is destructive, this is called a "fade", and the received signal is attenuated. If the fading is flat over the entire transmission bandwidth, this is known as frequency non-selective fading (i.e., relative to the bandwidth). If the fading characteristics vary over the transmission bandwidth, this is known as frequency selective fading.

Figure 2-1: Multi-path Propagation

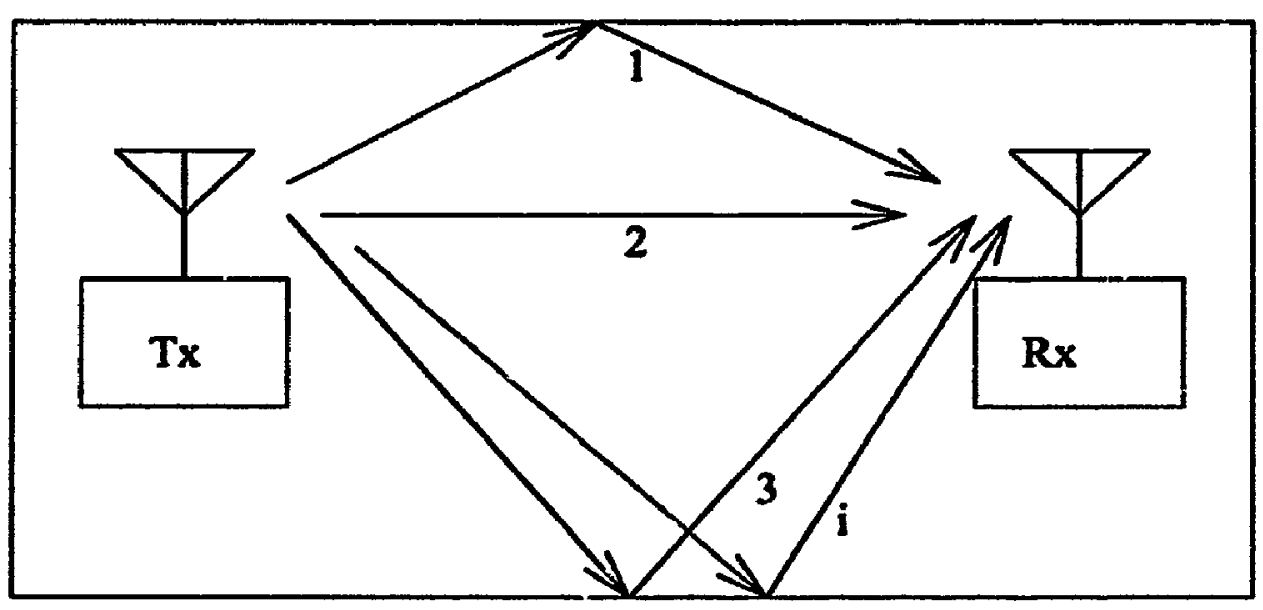


The indoor sadio channel presents a severe multi-path propag tion problem (especially for mobile handsets), and fades occurring at a particular frequency can last from several seconds to several minutes. For high data rate systems, such fades result in a significant loss of transmitted data unless counter measures are taken to combat the multi-path effects.

The impulse response, $h_{i}(t)$, for the ith propagation path shown in Figure (2-1) is given by;

$$
h_{i}(t)=\rho_{i}(t) \delta\left(t-\zeta_{i}(t)\right) \mathrm{e}^{j \phi_{i}(t)}
$$

Where $p_{i}(t)$ is the Rayleigh distributed and independent transmittance of the ith path, $\delta$ is the delta function, $\zeta_{\mathrm{i}}(t)$ is the uniformly distributed and independent delay of the ith path, and $\phi_{\mathrm{i}}(t)$ is the random phase of the ith path and is uniformly distributed between 0 and $2 \pi$.. The transmittance, delay, and phase of each path are functions of time, but for slow fading environments they are often considered to be time-invariant over the transmitted data symbol period [Pro83]. The received signal, $s(t)$, is given by;

$$
s(t)=\sum_{i=1}^{N} \rho_{i}(t) A \cos \left(2 \pi f_{c} t-2 \pi \zeta_{i}(t)+\phi_{i}(t)\right)
$$

Where $A$ is the amplitude of the transmitted signal, and $f_{c}$ is the carrier frequency. An important characteristic of any multi-path environment is the degree of correlation between separate paths. If two or more paths are highly correlated, then signals travelling along these paths will undergo almost identical channel effects. If the correlation between two or more paths is low, then signals travelling along these paths will undergo different channel effects. Two important functions are derived using these two principles, the first being the spaced-frequency correlation function, and the second buing the spaced-time correlation function [Pro83]. From the spaced-frequency correlation function the mean delay-spread, $\tau_{m}$, is obtained. The mean delay-spread is basically the expected value of the delays for all 
of the separate paths. The inverse of the mean delay-spread is salled the "coherencebandwidth" of the channel. From the spaced-time correlation function, the doppler-spread of the channel, $B_{d}$, is obtained. The inverse of the doppler-spread is called the "coherencetime" of the channel. If two transmitted sinusoids are separated in frequency by an amount greater than the coherence-bandwidth of the channel, they will undergo separate multi-path fading effects. If two sinusoids are transmitted with a time difference greater than the coherence-time of the channel, they will undergo separate inuliti-path effects. The coherence-time and the coherence-bandwidth of a channel are the basis for systems that make use of frequency-diversity and time-diversity. In a system that uses frequencydiversity to combat multi-path effects, the transmitter transmits the same data at as many different carrier frequencies as possible, all of which are separated in frequency by an amount greater than the coherence-bandwidth. The situation is similar for time-diversity, except that the same data is transmitted at different times separated by an amount greater than the coherence-time of the channel. The rcieiver then combines the information from the separate paths before making a decision as to whether a one or a zero was transmitted.

Measurements of the mean delay-spread for various indoor environments have been performed [SV87]. The results show that the delay-spread in a typical building can range anywhere from $50 \mathrm{~ns}$ to $\mathbf{2 0 0} \mathrm{ns}$. This means that in order for a radio system to make use of frequency-diversity in these environments, the transmitted signals should be separated in frequency by an amount at least greater than the inverse of the largest measured delayspread.

\subsection{Wireless Radio Standards}

With the emergence of several new international transmission standards and modulation formats, choosing an appropriate method of transmission for a given application has become a difficult challenge. Some of these standards and their requirements are summarized in Table (2-1). Of particular interest to mobile radio designers are the two FCN bands, CT-2, and DECT. 
Table 2-1: Wireless Transmission Standards

\begin{tabular}{|l|l|l|l|}
\hline & \multicolumn{1}{|c|}{ Europe DECT } & \multicolumn{1}{|c|}{$\begin{array}{l}\text { Europe Cellular } \\
\text { Mobile GSM }\end{array}$} & \multicolumn{1}{|c|}{ England CT-2 } \\
\hline $\begin{array}{l}\text { 1. RF frequency } \\
\text { band (MHZ) }\end{array}$ & $1800-1900$ & $\begin{array}{l}890-915 \\
935-960\end{array}$ & $\begin{array}{l}864.1-868.1 \\
40 \text { Channels }\end{array}$ \\
\hline $\begin{array}{l}\text { 2. Channel } \\
\text { Bandwidth }\end{array}$ & $1728 \mathrm{KHz}$ & $\begin{array}{l}200 \mathrm{KHz} \\
\text { (interleaving?) }\end{array}$ & $100 \mathrm{KHz}(?)$ \\
\hline 3. Bit rate & $1152 \mathrm{~Kb} / \mathrm{s}$ & $270.833 \mathrm{~Kb} / \mathrm{s}$ & $72 \mathrm{~Kb} / \mathrm{s}$ \\
\hline $\begin{array}{l}\text { 4. Spectral effi- } \\
\text { ciency }\end{array}$ & $0.67 \mathrm{~b} / \mathrm{s} / \mathrm{Hz}$ & $1.35 \mathrm{~b} / \mathrm{s} / \mathrm{Hz}$ & $0.72 \mathrm{~b} / \mathrm{s} / \mathrm{Hz}$ \\
\hline 5. Access method & TDM/t & TDMA & TDMA (?) \\
\hline $\begin{array}{l}\text { 6. Mobile RF } \\
\text { power/out-of-band }\end{array}$ & $\begin{array}{l}250 \mathrm{~mW} \\
\text { /-35 dBr }\end{array}$ & $\begin{array}{l}20 \mathrm{~W}(43 \mathrm{dBm}) \\
\text { /-33 cBr }\end{array}$ & /-dBr (?) \\
\hline $\begin{array}{l}\text { 7. Modulation } \\
\text { GFSK }\end{array}$ & $\begin{array}{l}\text { GMSK } \\
\text { BT=0.3 }\end{array}$ & $\begin{array}{l}\text { GMSK } \\
\text { BT=? }\end{array}$ \\
\hline 8. Demodulation & $\begin{array}{l}\text { Coherent or nonco- } \\
\text { herent (?) }\end{array}$ & $\begin{array}{l}\text { Coherent } \\
\text { (Probably) }\end{array}$ & $(?)$ \\
\hline $\begin{array}{l}\text { 9. Adaptive } \\
\text { Equalization }\end{array}$ & No (?) & YES & $(?)$ \\
\hline 10. Performance & not too stringent & stringent & $(?)$ \\
\hline 11. Market size & $\begin{array}{l}50-70 \text { million } \\
1995 ? ?\end{array}$ & $\begin{array}{l}15 \text { million (?) } \\
1995\end{array}$ & $? ?$ \\
\hline
\end{tabular}

Three additional frequency bands have recently been opened by the FCC that allow either frequency-hopping or direct-sequence spread-spectrum transmission to be used. These bands are located at $902-928 \mathrm{MHz}, 2.4-2.4835 \mathrm{GHz}$, and $5.725-5.85 \mathrm{GHz}$, and allow up to 1 Watt of transmitted power [Lef94]. For frequency-hopping transmission, the carrier frequency must be hopped at least once every $400 \mathrm{mS}$. 


\subsection{Trends in VLSI for Wireless Communications}

As VLSI technologies continue to progress the possibility of a fully integrated radio with both the RF front-end components and digital circuitry co-existing on the same chip is becoming feasible. What was once believed to be a fundamental physical limit on transistor geometry size has been exceeded, and processes with 0.35 um feature sizes and smaller are under development [NTR94]. BiCMOS processes mix both MOS devices and bipolar devices on the same chip, providing designers with the performance and low power consumption of the MOS devices for digital designs, while offering access to the analog advantages and high drive capabilities of bipolar devices. Advances in silicon-germanium technologies are producing bipolar devices that have cut-off frequencies of $50 \mathrm{GHz}$ and higher, making them ideal candidates for mobile radio applications in the 1 to $6 \mathrm{GHz}$ frequency range.

However, as feature sizes continue to decrease it has been found that while digital circuits scale easily, operating more quickly and consuming less power, analog circuits suffer from degraded signal-to-noise ratio (SNR), and increased power consumption for certain designs. These problems have led to communication ciriuit designers moving the system complexity from the analog side to the digital side where possible, avoiding the scaling problems inherent in the analog circuits, and taking full alvantage of smaller geometry digital circuits [TA85]. This approach also allows significant portions of the circuit layout to be produced using high-level digital synthesis tools.

Digitization of received signals is being performed closer to the frontend of digital radio systems, and digitization at the IF frequency is becoming more common, with hopes of eventually quantizing the received signal directly at RF without any initial frequency down-conversion required. 


\subsection{Frequency-synthesis}

Frequency-synthesis is one of the most important functions performed in telecommunication systems. Frequency synthesizers are used to generate the signals required in radio systems for the carrier frequency, and the local oscillator signals used for frequency translation. The most important properties of frequency synthesizers are frequency-range, frequency-resolution, settling-time, phase-noise, spurious-sontent, and modulation potential. For mobile radio applications, small size, and low power consumption are also a major concern. The three basic methods of frequency-synthesis can be classified as direct-analog, direct-digital, and indirect-analog synihesis. The following sections describe these three methods of synthesis in greater detail.

\subsubsection{Direct-analog Frequency-synthesis}

In direct-analog frequency-synthesis (DAS), the desired output signal is obtained using one or more reference frequencies, and using various analog components, such as mixers, dividers for frequency translation, and switches. Filters are used to remove unwanted tones from the system. An example of a direct-analog frequency-synthesizer is shown in Figure (2-2).

Figure 2-2: Direct-analog Frequency Synthesizer

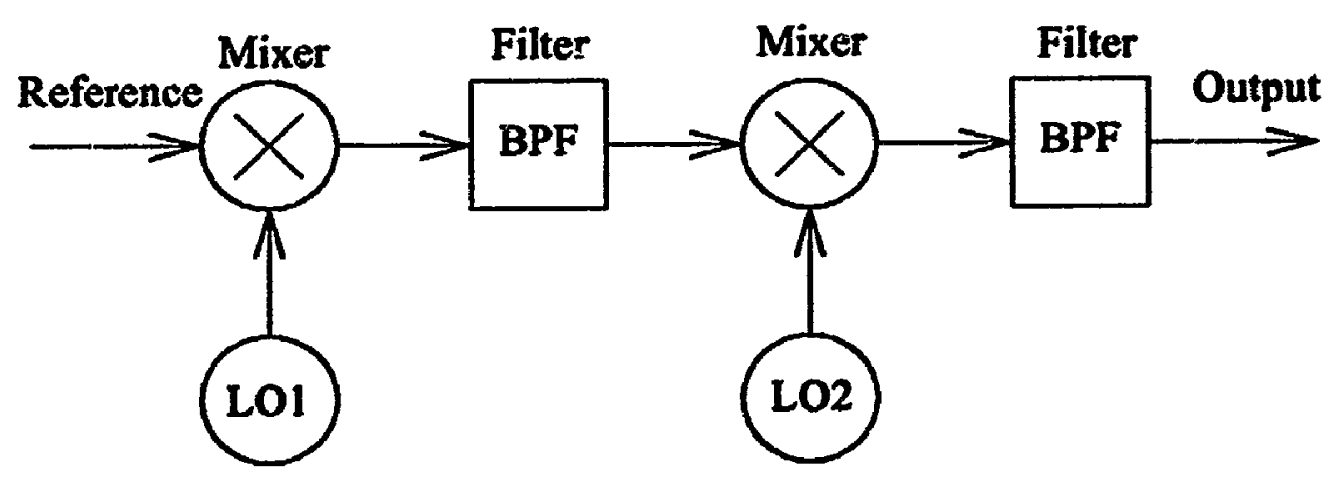


The range of frequencies that can be produced using DAS techniques is very wide, as almost any signal can be generated if the appropriate reference frequencies and translation stages are employed. Signals generated by DAS systems can be easily modulated through the use of mixers. The frequency-resolution of the system is determined by the reference signals used. DAS systems suffer from phase-drift over time, and because of this, periodic frequency adjustments must be made to these systems. Because the mixers used in DAS systems are inherently non-linear circuit elements, spurious tones are generated within these systems and must be controlled through the use of filters. Many of the filters used to eliminate spurs in DAS systems have narrow bandwidths, and therefore introduce unwanted distortion in the output spectrum of the synthesizer, as well as limiting the frequency settling-time. DAS systems have the drawback of being made up of many complex analog circuits which use discrete components that are very difficult or impossible to integrate, making DAS systems unsuitable candidates for mobile radio applications.

\subsubsection{Direct-digital Frequency-synthesis}

A block diagram of a frequency svnthesizer that makes use of direct-digital synthesis (DDS) techniques is presented in Figure (2-3).

\section{Figure 2-3: Direct-digital Frequency Synthesizer}

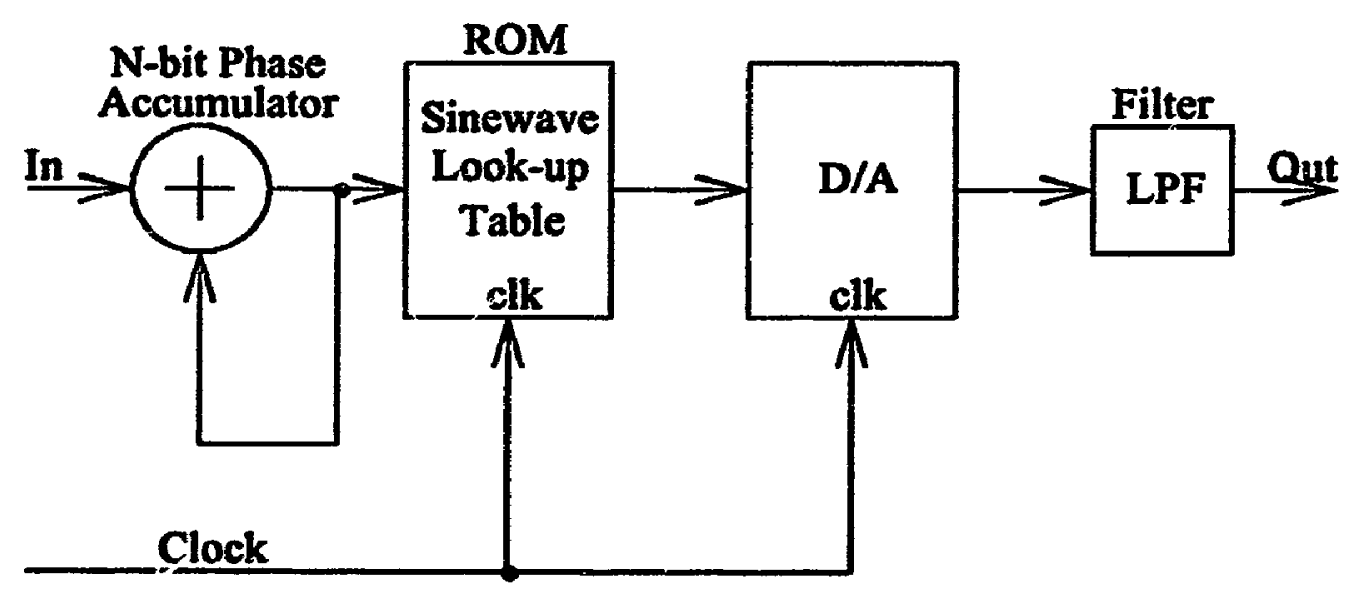


The system works as follows. A digital-reference frequency is accumulated in order to obtain instantaneous-phase information which is used to address a ROM based look-up table that contains the appropriate sinewave amplitudes [Don92]. The digital word obtained from the table is then converted to an analog signal using a D/A converter. The performance of DDS systems is limited by the wordlength of the look-up table used, the D/A converter, and subsequent filtering of the output. In order to obtain high frequency-resolution and low spurious-content, the digital complexity and dynamic range required is high, as well as expensive. The maximum output frequency for direct-digital synthesis is limited to frequencies below the Nyquist rate, which is one half the clock frequency used in the synthesizer. As VLSI technologies continue to progress, DDS techniques are becoming more popular because of the small size and low power consumption that can be obtained through all digital design. However, for most mobile radio applications, DDS systems require an extra frequency upconversion stage in order to obtain the required output frequency, adding more analog complexity to the system and degrading the overall performance.

\subsubsection{Indirect-analog Frequency-synthesis}

Indirect-analog synthesis techniques use feedback control in order to phase-lock, or frequency-lock, a voltage-controlled-oscillator $(\mathrm{VCO})$ to a stable reference frequency. A block diagram of a simple PLL synthesizer is shown in Figure (2-4). 
Figure 2-4: Phase-locked loop Frequency Synthesizer

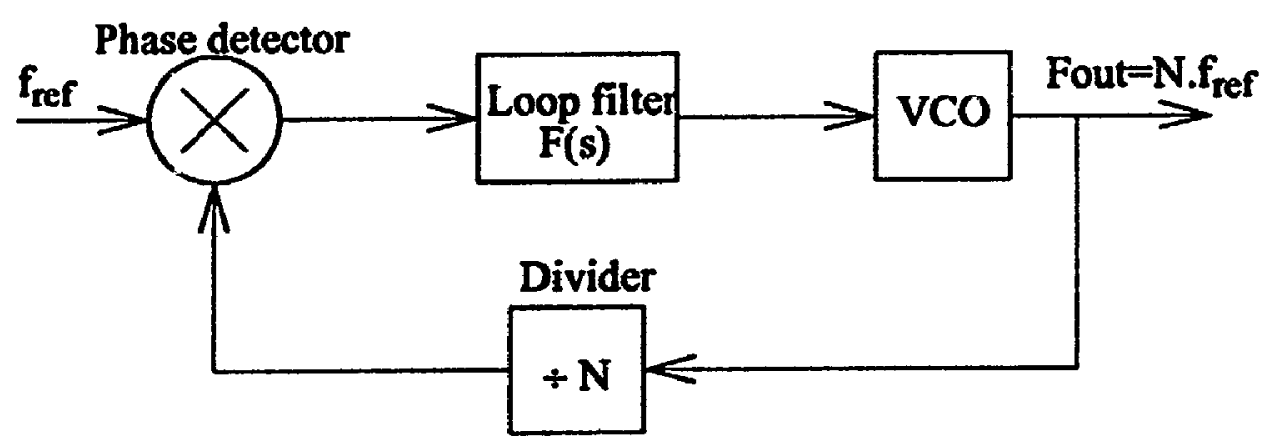

The reference frequency, $f_{r e f}$ is phase compared to the divided output frequency of the VCO. The signal generated by the phase detector has an average value which is proportional to the phase difference between the two frequencies. The error signal is then filtered in order to obtain a control signal that is used to pull the output frequency of the VCO in the direction required to reduce the phase-error. The transient properties of the loop are determined by the properties of the loop filter, $F(s)$. The frequency-range of the PLL is limited by the tunability of the VCO used, though centre frequencies of $1 \mathrm{GHz}$ or more are common. The frequency-resolution of most standard PLL synthesizers is limited to integer multiples of the reference frequency, although this restriction does not apply to fractional$\mathbf{N}$ synthesizers. The settling-time of a PLL synthesizer is inversely proportional to the closed-loop bandwidth of the PLL. Since the phase-noise level anci modulation potential of the loop are also determined by the closed-loop bandwidth, a great deal of design optimization is required in order to balance these three requirements. 
A block diagram of a fractional- $\mathrm{N}$ frequency synthesizer is given in Figure (2-5).

Figure 2-5: Fractional-N PLL Based Frequency Synthesizer

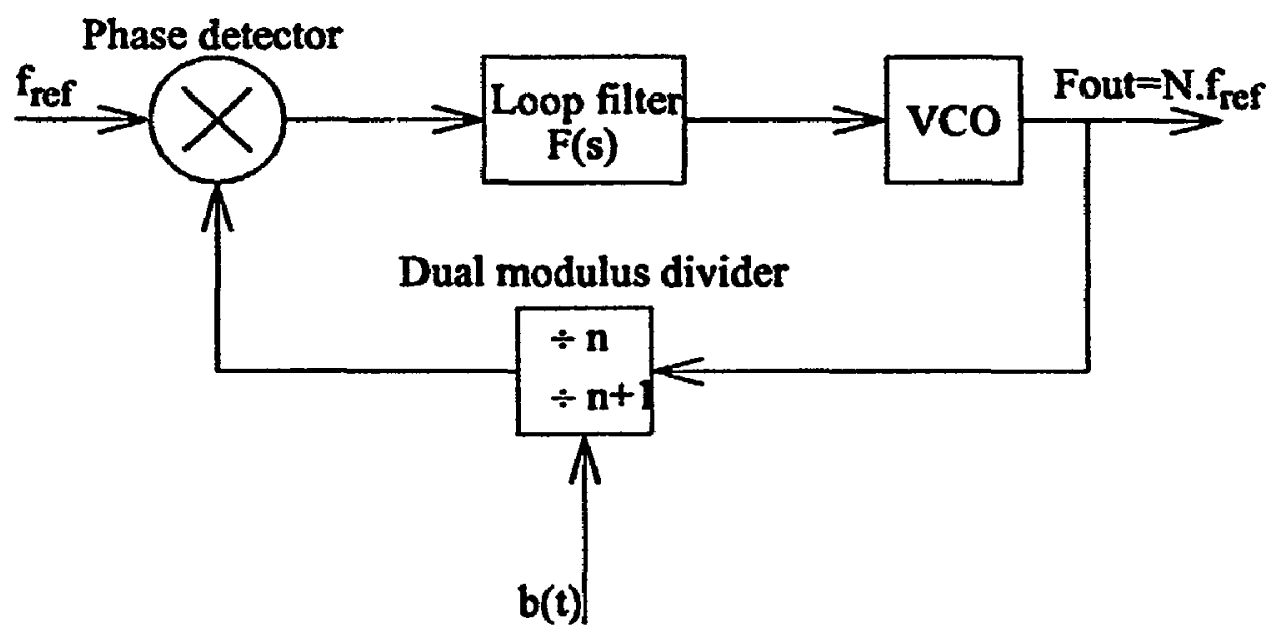

Control Bitstream

This type of synthesizer uses a dual-modulus divider in the feedback path. The divider divides by $\mathrm{n}$ or $\mathrm{n}+1$ depending on the value of the control signal shown in Figure (2-5). The output frequency of the synthesizer is equal to $N . f_{\text {ref, }}$, where $\mathbf{N}$ is the numerically-averaged division-ratio, and can have a fractional value. The most difficult aspect of designing a fractional- $N$ synthesizer is generating a suitable control bitstream, $b(t)$, for the divider. The control bitstream must have an average value equal to $\mathrm{K}$, a digital word that represents the desired output frequency of the synthesizer. However, $b(t)$ contains periodic components other than the desired division ratio, resulting in spurious tones generated in the PLL. Considerable effort must be spent by the designer to ensure that these unwanted tones fall outside of the closed-loop bandwidth of the PLL. . s common method used to generate the divider control signal is to use the overflow output from a K-bit digital accumulator [Don92]. Spurious-tones can be reduced by randomizing the resulting bitstream through the injection of random digital words at the input of the accumulator. However, this form of "dithering" raises the overall noise floor of the PLL [Don92]. Most of the components used in PLL based frequency synthesizers are suitable for monolithic integration, making indirect-analog frequency synthesis an attractive solution for systems that require 
synthesizers with small size, and low power consumption. A recent study has shown that for mobile radio applications that require agile frequency synthesizers and high frequencyresolution, the most suitable synthesis method is fractional-N, using $\Delta-\Sigma$ modulation methods to generate the divider control bitstream [Bax93]. The use of $\Delta-\Sigma$ techniques pushes the resulting quantization noise outside of the closed-loop bandwidth, greatly reducing output spurious content, and maintaining a low noise floor within the loop bandwidth [RCK93].

\subsection{Phase-error in Phase-locked Loops}

One of the most important aspects of PLL analysis is determining the response of a particular loop to a change in the input signal. Any disturbance of the input to the loop results in transient phase-error being generated. If the disturbance is larger than the tracking range of the loop, loss of lock may occur. Loss of lock occurs due to limitations in phasedetector linear range, allowable voltage levels within the loop, VCO tuning range, etc. Provided that the loop remains locked after an input change, linear analysis methods can be used to determine the value of the phase-error. If the phase-error transfer function for a loop is found, it can be used to determine the phase-error generated by a particular change in input. The phase-error transfer function for the loop that was shown in Figure (2-4) is given by;

$$
\frac{\theta_{e}(s)}{\Delta \omega_{i}(s)}=\frac{1}{s+K F(s)}
$$

Where $\theta_{\mathrm{e}}(\mathrm{s})$ is the output phase-error, $\Delta \omega_{\mathrm{i}}(\mathrm{s})$ is the change in input frequency, $\mathrm{K}$ is the open-loop gain, and $F(s)$ is the transfer function of the loop filter. For the purposes of the research performed in this thesis, the response of a PLL to a step in input frequency, and the response to random frequericy modulation of the input, are two cases that are of particular interest. 


\subsubsection{Phase-error Due to a Frequency Step}

A frequency step, $\Delta \omega_{i}(t)$, is defined in the time domain as;

$$
\Delta \omega i(t)=\Delta \dot{\omega} u(t)
$$

Where $\Delta \omega$ is the magnitude of the frequency step, and $u(t)$ is the unit step function. Taking the Laplace transform of the above expression we obtain;

$$
\Delta \omega i(s)=\frac{\Delta \omega}{s}
$$

Making use of Equations (2-3) and (2-5) we find that the phase-error generated in the loop shown in Figure (2-4) due to a step in input frequency is given by;

$$
\theta_{e}(s)=\frac{\Delta \omega}{s(s+K F(s))}
$$

The inverse Laplace transform of Equation (2-6) is the tinse domain expression for the phase-error generated in the loop due to a step in input frequenc; of magnitude $\Delta \omega$. If the loop filter of the PLL shown in Figure (2-4) is an integrator with phase-lead correction, the filter transfer function is given by;

$$
F(s)=\frac{\left(1+\tau_{2} s\right)}{\tau_{1} s}
$$

Where $\tau_{1}$ and $\tau_{2}$ are the filter time constants. Figure (2-6) shows plots of the transient phase-error generated by a step in input frequency, for the loop described above [Bla92]. 
Figure 2-6: Transient Phase-error in PLC Due to a Step in Input Frequency

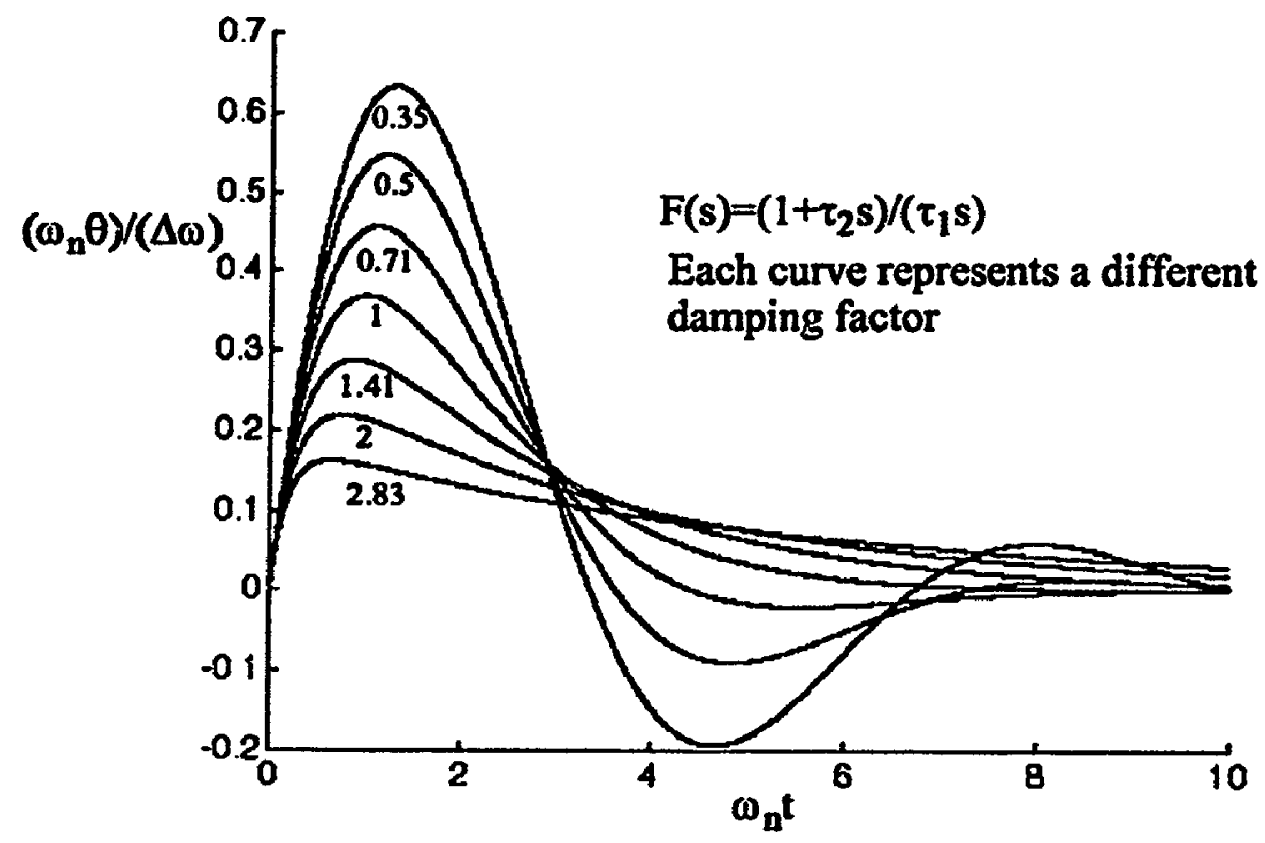

\subsubsection{Response of a PLL to Random FM Modulation}

Using the phase-error transfer function for a PLL, it is possible to determine the root-meansquare (rms) phase-error generated in a loop due to random frequency modulation of the input. Given the statistical properties of the modulating waveform, its spectral density can be found. The spectral density for the phase-error, $\Phi_{\mathrm{e}}(\mathrm{s})$, generated in the loop is given by;

$$
\Phi_{e}(s)=\left|\frac{\theta_{e}(s)}{\omega i(s)}\right|^{2} \Phi_{f m}(s)
$$

Where $\Phi_{\mathrm{fm}}(\mathrm{s})$ is the spectral density of the random modulating waveform, and the term in the brackets is the frequency input to phase-error output transfer function. The mean-square value of $\theta_{\mathrm{e}}(\mathrm{s})$ is given by; 


$$
\vec{\theta}_{e}^{2}=\int_{0}^{\infty} \Phi_{e}(s) d f
$$

If we take the square-root of both sides of the above equation, we obtain the rms phase-error generated in the loop due to the random frequency modulation of the loop input.

\subsection{Continuous-phase Modulation Tecïniques}

When digital data is transmitted using unfiltered frequency-shift-keying (FSK) or unfiltered phase-shift-keying (PSK) modulation, the resulting transmitted spectrum is wideband in nature due to the frequency or phase discontinuities present [LW90]. Continuous-phase modulation (CPM) techniques use baseband filtering to shape the transmitted data symbols in order to obtain improved spectral characteristics, such as smaller bandwidth occupancy, lower sidelobe energy, and reduced error probability in the receiver [Sun86], [KS75]. CPM introduces memory into the baseband system as the phase of the filtered signal is continuous from symbol to symbol, regardless of the symbol values. The optimum detector for CPM is phase-coherent, and observes the signal over several symbol intervals before making a bit decision. If the baseband pulse is one symbol interval in duration, this is known as full response signaling (FRS). If the baseband pulse is stretched over more than one symbol interval, this is known as partial response signaling (PRS). By allowing a controlled amount of inter-symbol interference (ISI) to be present in the system, PRS allows smoother phase transitions and a narrower transmitted spectrum than FRS [KS75]. Using PRS techniques, nulls can be placed in the data spectrum in order to reduce undesired ISI, and the smooth phase transitions obtained make the system less sensitive to timing errors [KS75]. A PRS system can be modelled by the block diagram presented in Figure (2-7) [KS75]. 


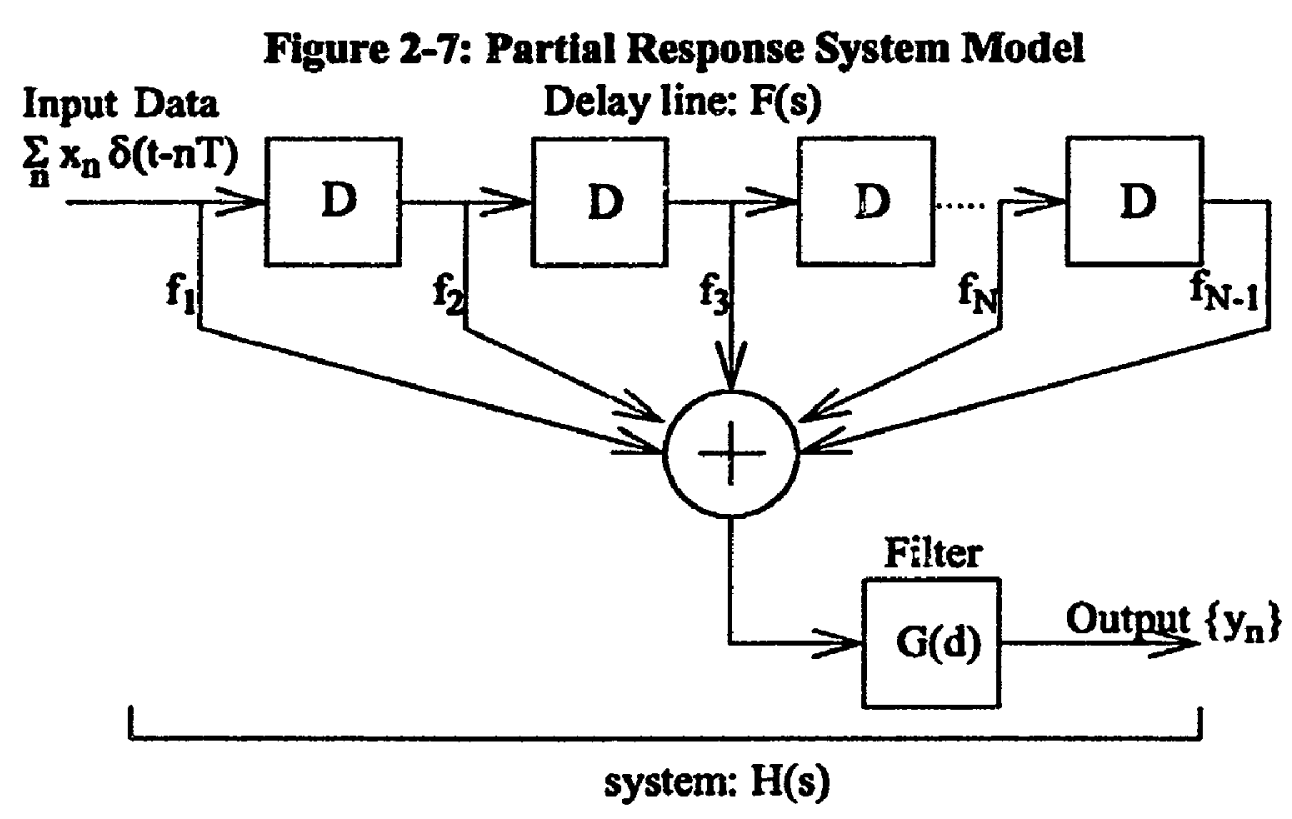

The overall impulse response of the system shown in Figure (2-7) is $h(t)$, and the impulse response of $G(d)$ represents the desired baseband pulse shape. The blocks labelled with a $D$ represent time delays of one symbol period each, and $d$ represents a delay equal to the inverse of the oversampling rate used in the digital filter $G(d)$. The PRS system polynomial, $F(D)$, is given by;

$$
F(D)=\sum_{n=0}^{N-1} f_{n} D^{n}
$$

Where $f_{n}$ are the system coefficients, and $N$ is the number of symbols combined in the filter [KS75]. For an input sequence $\left\{x_{n}\right\}$ the output of the system is given by;

$$
Y(D)=X(D) F(D)
$$


where $X(D)$ is;

$$
X(D)=\sum_{n=0}^{\infty} x_{n} D^{n}
$$

and

$$
Y(D)=\sum_{n=0}^{\infty} y_{n} D^{n}
$$

where $x_{n}$ is the value of the $n$th data symbol and $y_{n}$ is the value of the nth output. Different spectral properties for the transmitter signal can be obtained through careful selection of the system polynomial, and the baseband pulse shape [KS75].

Figure (2-8) shows how three symbols are combined in a system with a characteristic polynomial given by;

$$
F(D)=1+D+D^{2}
$$

and a Gaussian baseband pulse truncated symmetrically to three symbol periods [RC94]. The output of the filter, shown in Figure (2-8) is only specified for the time interval from 0 to $T$. 
Figure 2-8: Symbol Combining in a PRS System

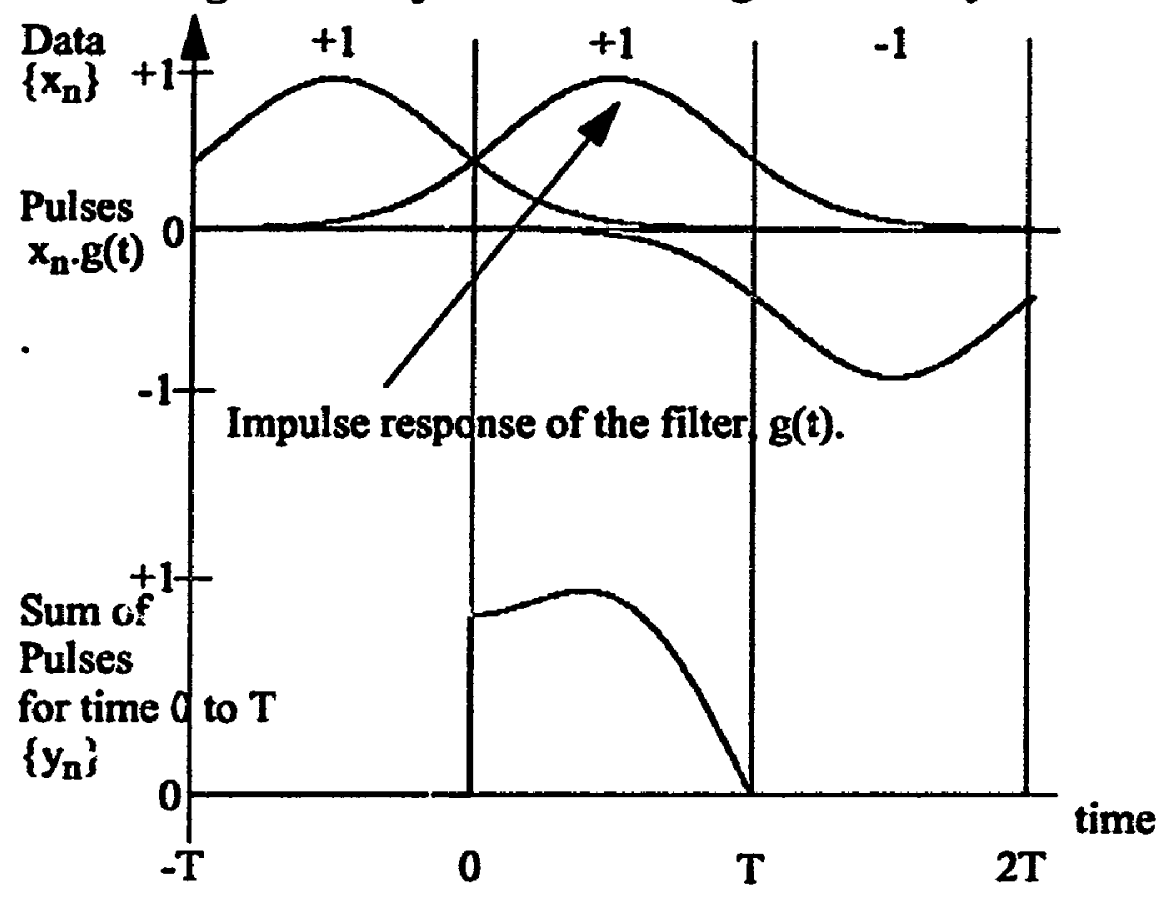

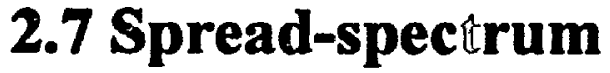

Spread-spectrum, used by the military for over thirty years [Sch82], has become of interest for use in commercial mubile-radio applications. In spread-spectrum, a signal is spread over a frequency bandwidth that is much wider than the minimum bandwidth required to transmit the signal. This spreading is accomplished using a pseudo-random code that is unrelated to the transmitted data. Frequency spreading offers the transmitted signal several advantages over stationary carrier transmission, such as resistance to jamming, resistance to interference and multi-path fading, low probability of intercept, improved privacy, and the possibility of using code-division multiple access [PSM82]. For commercial applications resistance to deliberate jamming and low probability of intercept are of little concern. 


\subsubsection{Processing-gain}

The anti-jamming and low interference properties of direct-sequence spread-spectrum transmission are inherent in the despreading process that takes place in the receiver. The signal-to-noise advantage that is obtained through this process is called the "processinggain" of the system and is common to most forms of spread-spectrum. Full mathematical descriptions of processing-gain have been presented in spread-spectrum literature and only a brief explanation will be presented here. Figure (2-9 a) shows the frequency spectrum of a transmitted spread-spectrum signal along with a finite energy interferer. In the receiver the signal is despread and the resulting frequency spectrum is shown in Figure (2-9 b).

Figure 2-9: Processing-gain

a)

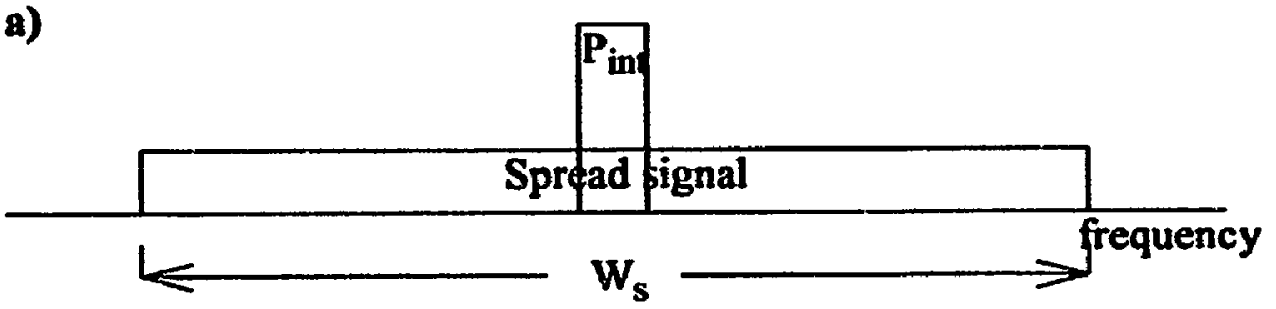

b)

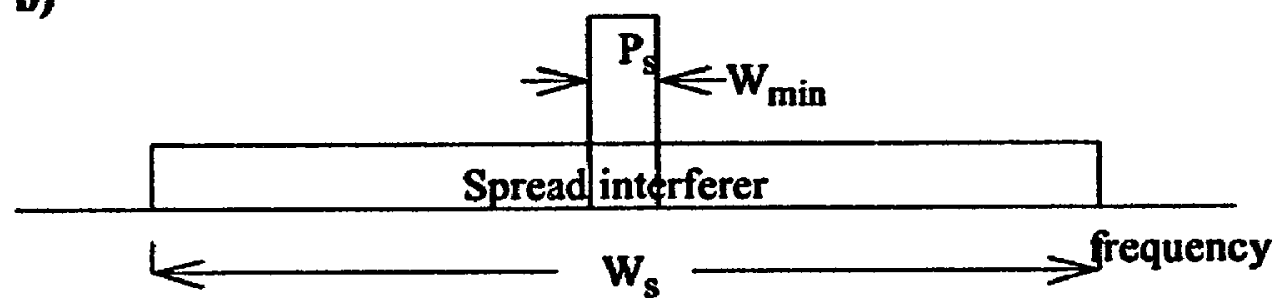

It can be seen from Figure (2-9 b) that while the transmitted signal has been compressed back to its original bandwidth by the :spreading process, the interfering signal has been spread over the spread-spectrum bandwidth. The power spectral density of the interfering signal, $\mathbf{S}_{\text {int }}$ after spreading, is given by; 


$$
S_{i m \ell}=\frac{P_{i n t}}{W_{s}}
$$

Where $P_{\text {int }}$ is the total power of the interferer, and $W_{\mathbf{s}}$ is the spreading bandwidth in $\mathrm{Hz}$. The recovered signal along with the spread interferer is then sent through a bandpass filter whose bandwidth is ideally equal to the bandwidth of the despread data signal. The signalto-noise ratio at the output of the filter is given by;

$$
S N R=\frac{P_{s}}{P_{\text {in }}}\left(\frac{W_{s}}{W_{\text {min }}}\right)
$$

Where $P_{s}$ is the power of the despread signal, and $W_{\min }$ is the minimum bandwidth required to transmit the signal. The term in the brackets is a measure of the processing-gain of the system. The recovered signal has a definite power advantage over the interfering signal at the output of the bandpass filter. This advantage is not present in the case of interfering signals that have theoretically infinite energy, such as additive-white-Gaussian-noise (AWGN). For such cases, spread-spectrum transmission offers no advantage over a stationary carrier.

\subsubsection{Forms of Spread-spectrum Transmission}

This thesis considers three basic kinds of spread-spectrum signals [RD77]. Frequencyhopping (FH), time-hopping (TH), and direct-sequence (DS). In a frequency-hopping system the carrier frequency is hopped around in a pseudo-random sequence which is known by both the transmitter and the receiver. In time-hopping, the data is transmitted in bursts that occur at pseudo-random times known by both the transmitter and the receiver. In direct-sequence spread-spectrum, the data is modulated by a high speed pseudo-random binary bitstream which spreads it over a frequency bandwidth that is determined by the pseudo-random code bit-rate. Again, the code is known by both the transmitter and the 
receiver.

Various hybrid forms of the three types of spread-spectrum transmission mentioned above exist, but are not covered in this thesis. An alternative form of spread-spectrum transmission has recently been presented in another thesis [Ken94], and is called broadband-coded FM. In broadband-coded FM, a pseudo-random waveform is used to FM modulate a carrier separately from the regular data modulation. The maximum frequency deviation of the modulating waveform is much higher than the modulation rate, resulting in a transmitted signal whose bandwidth occupancy is almost completely determined by the frequency deviation of the signal. The waveform used to modulate the carrier is known by both the transmitter and the receiver. In the receiver, the additional modulation is removed from the received signal prior to recovering the transmitted data.

Interpolated frequency-hopping (IFH), introduced in thesis, is a subset of broadband-coded FM and is closely related to conventional frequency-hopping. In interpolated frequencyhopping the frequency-hopping code is interpolated using a digital filter, resulting in a carrier that moves smoothly and continuously in frequency through a pseudo-random sequence known by both the transmitter and the receiver. Experimental results presented in Chapter 6 show that interpolated frequency-hopping tratssmission can reduce many of the problems inherent in phase-locked loop (PLL) based frequency-hopping, while offering similar advantages and some additional features. The following sections describe directsequence, frequency-hopping, and broadband-coded FM spread-spectrum in greater detail, and are followed by an analysis of interpol ated frequency-hopping presented in Chapter 3.

\subsubsection{Direct-sequence}

Block diagrams of a possible direct-sequence spread-spectrum transmitter and receiver are given in Figure (2-10). In the transmitter, data is modulo-2 added (XOR) to a high-speed pseudo-random binary bitstream. The resulting output bits are used to phase modulate the carrier frequency generated by the local oscillator. The code, data, and XOR output time domain waveforms are shown in Figure (2-11) along with the transmitted frequency 
spectrum. The signal is then upconverted to an RF carrier and transmitted. The transmitted data is spread around the carrier over a frequency bandwidth determined by the code bitrate. In order to recover the transmitted data, the receiver downconverts the signal and mixes it with a copy of the same code sequence used in the transmitter. The result of this operation is the IF frequency phase-modulated by the transmitted data bits. Conventional demodulation techniques can then be used to recover the baseband signal.

Often, in direct-sequence spread-spectrum receivers, a matched filter is used to despread the received signal [KM87]. The output of such a filter produces a separate correlation peak for each multi-path component of the signal that falls within the time resolution of the filter, as shown in Figure (2-12) [KM87].

Figure 2-10: Direct-sequence Spread-spectrum System

\section{Transmitter}

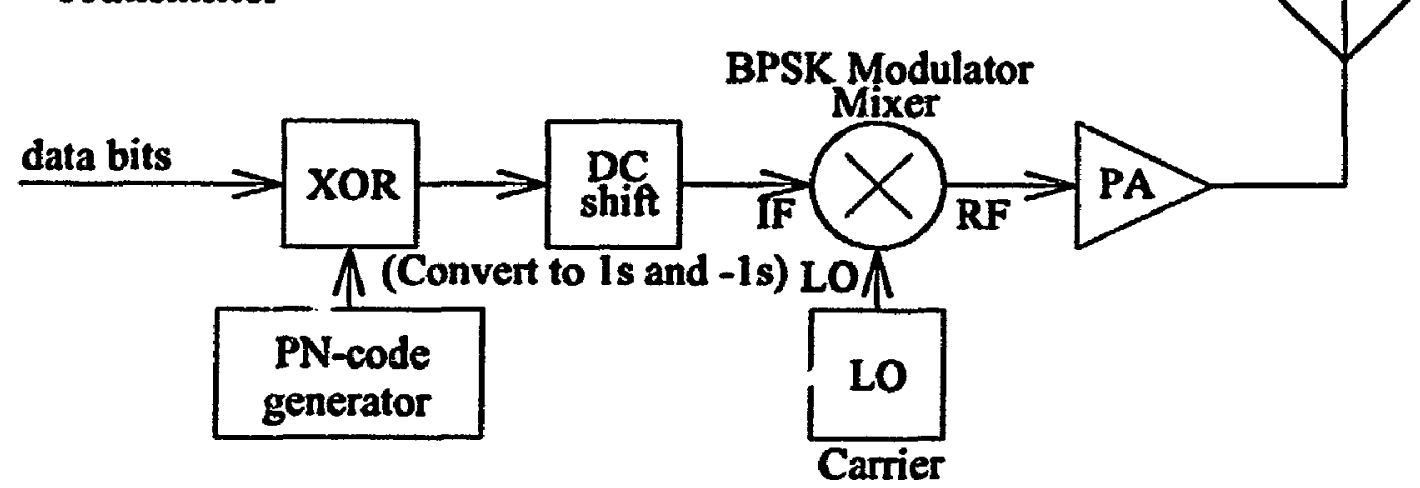

Receiver

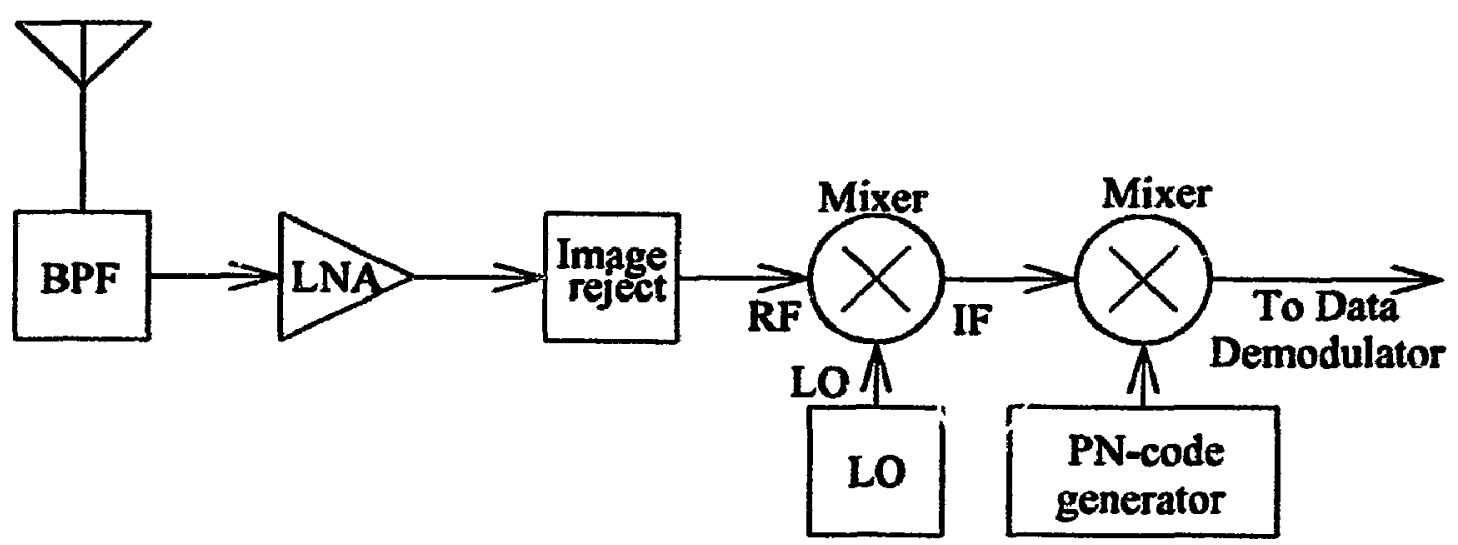


The time resolution of the matched filter is equal to the inverse of the bandwidth of the pseudo-random spreading code. The receiver can take advantage of this inherent timediversity by using selection-diversity techniques to select the largest peak, or combine several peaks, before making a bit decision.

Figure 2-11: Direct-sequence Spread-spectrum Waveforms
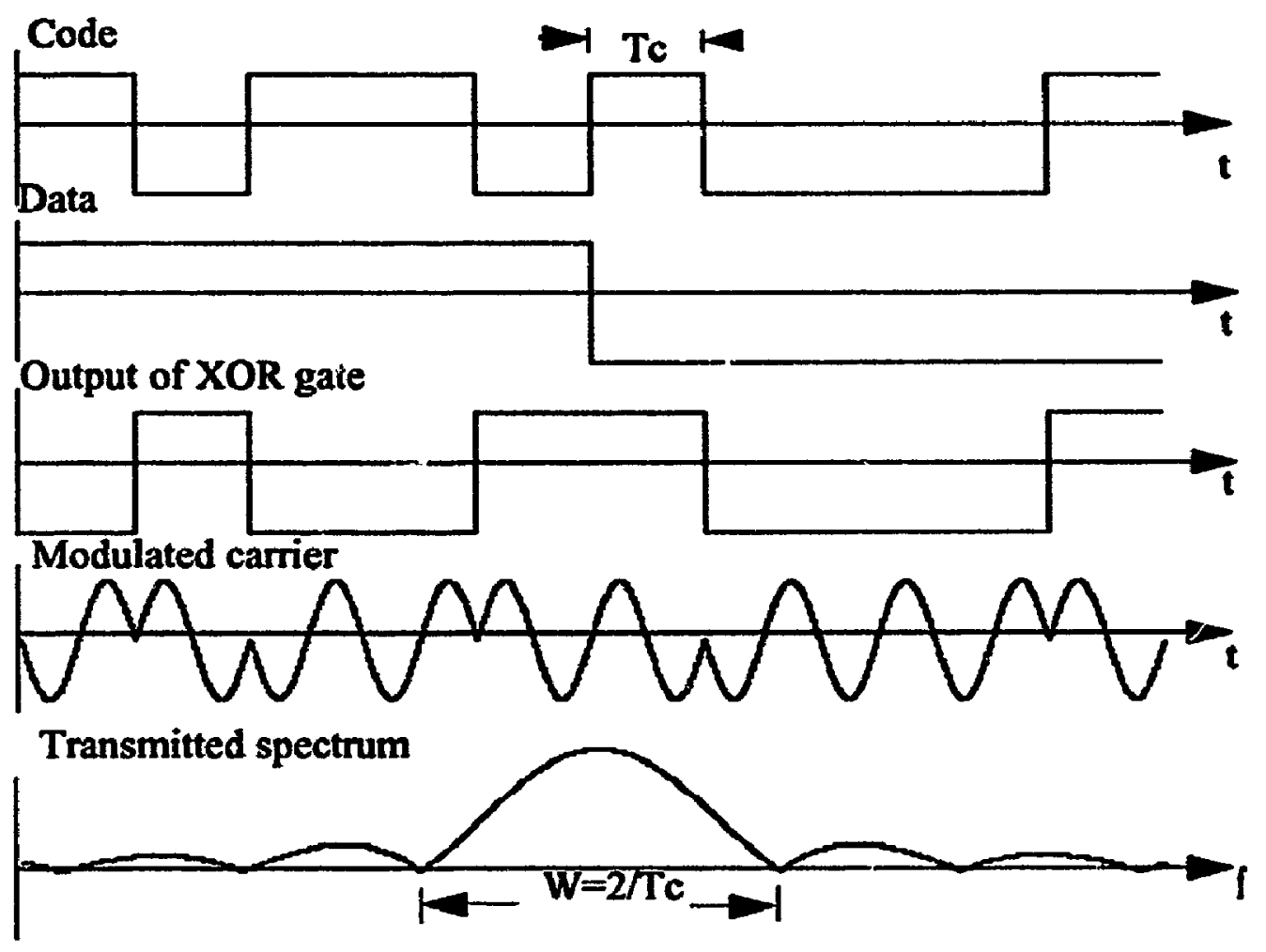
Figure 2-12: Matched filter for Direct-sequence Detection

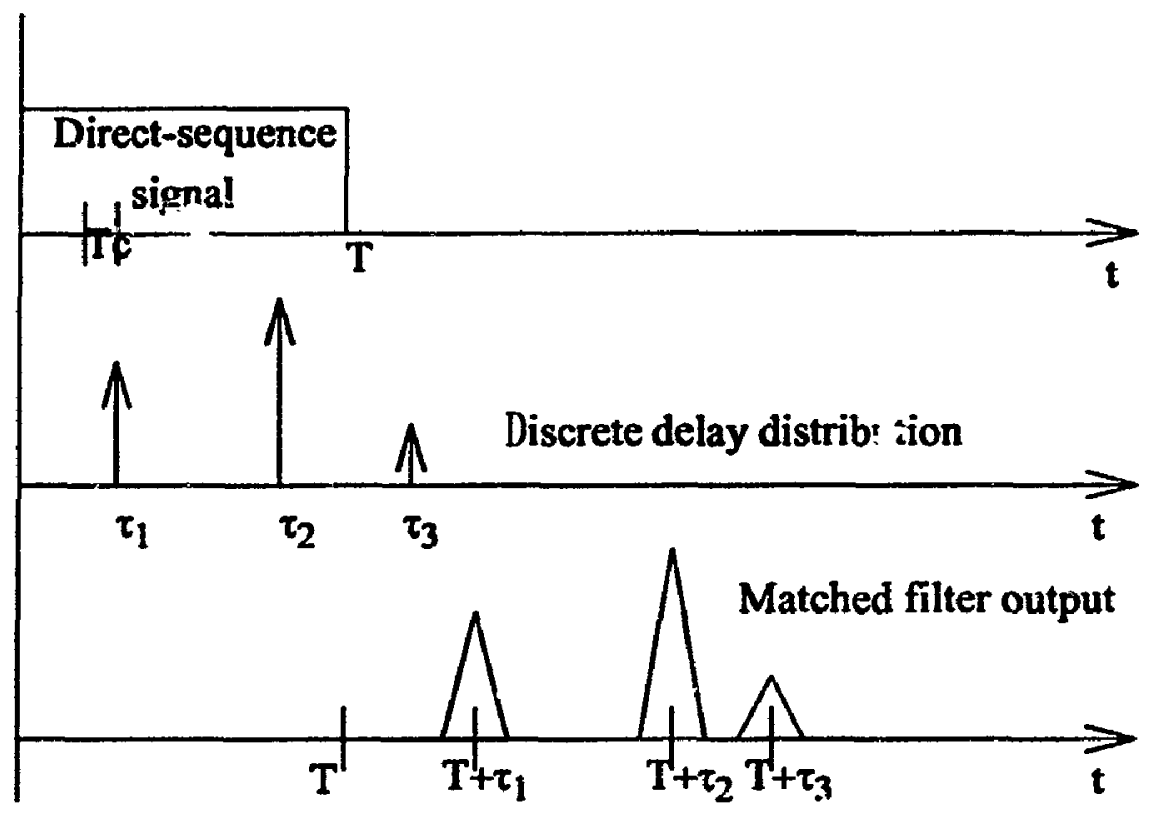

If several users are required to transmit simultaneously in the same environment without interfering with one another, CDMA transmission can be used. In direct-sequence CDMA each user in the system is assigned their own pseudo-random spreading code. Provided that the different codes used have low cross-correlation and low partial-correlation, each user will only receive the transmission meant for them, and the other users will appear as random noise to the receiver [Fis91]. As the number of users in the system increases, the level of the noise floor for the channel rises, and eventually reception becomes impossible. However, in direct-sequence CDMA, this transition is not as abrupt as in frequencydivision multiple-access (FDMA), or time-division multiple-access (TDMA). A problem encountered in direct-sequence CDMA systems is that any transceivers that are operating in close proximity to one another will saturate each others front-ends with noise, unless some form of adaptive gain control is used in the low-noise amplifier (LNA) of each receiver. This poses a challenge to system designers and is known as the "near/far effect " [Ker92]. 
Most of the signal-processing required in direct-sequence spread-spectrum systems is well suited for dighital techniques. DDS techniques are normally used to generate directsi quence signals as DAS techniques are too large and complex for use in mobile radio applications, and PLL based synthesizers in general do not have large enough modulation bandwidths to accommodate high code rates. In order to generate high RF centre frequencies, direct-sequence systems require an additional analog frequency upconversion stage. The timing requirements for code-phase synchronization between the transmitter and receiver in direct-sequence systems are quite stringent, and the received and locally generated codes must be aligned to within a fraction of a code bit period. 


\subsubsection{Frequency-hopping}

Figure (2-13) shows the blocik diagrams of a typical frequency-hopping transmitter and receiver.

Figure 2-13: Frequency-hopping Spread-spectrum System

Transmitter

FM modulated input data

Receiver

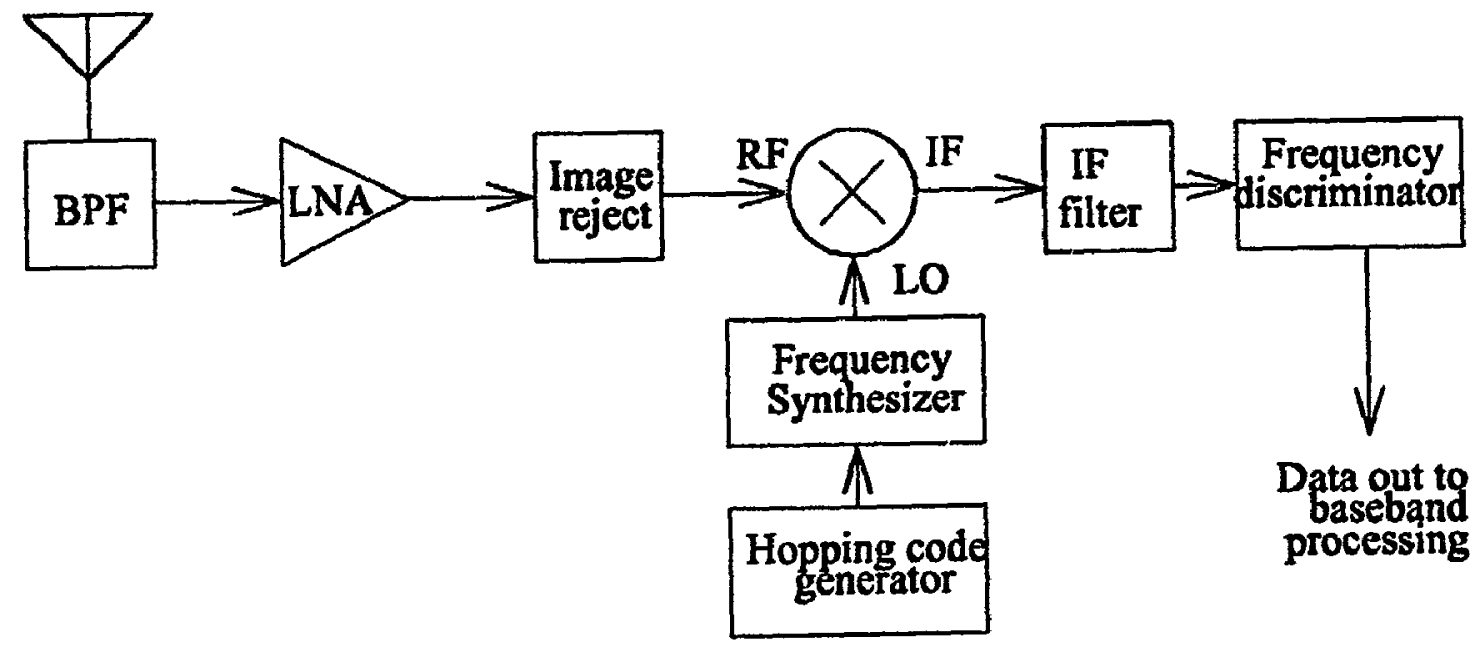

In the transmitter, a pse-do-random hopping code is used to control the output frequency of a phase-locked loop (PLL) based synthesizer. The carrier is then modulated by data and transmitted. An example of a frequency-hopping signal (frequency versus time), is shown in Figure (2-14). 


\section{Figure 2-14: Frequency-hopping Spread-spectrum}

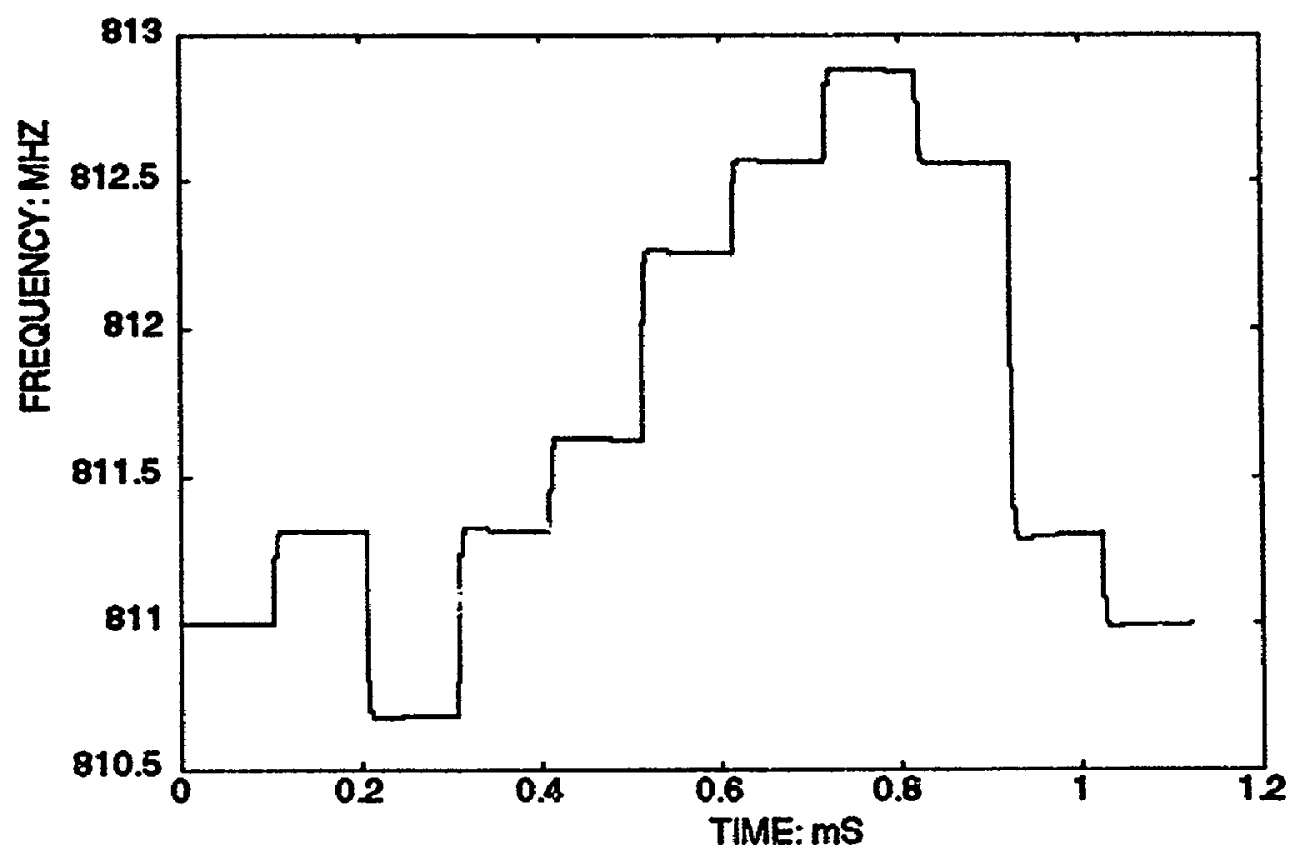

In the receiver, a copy of the frequency-hopping pattern plus an intermediate frequency (IF) offset is subtracted from the incoming signal in the frequency domain (i.e., by mixing). If the received signal is the desired one, and the transmitter and receiver are properly synchronized, the result of the subtraction is the FM carrier with data, centered at the IF frequency.

Since the carrier is regularly being hopped in frequency, frequency-hopping provides resistance to stationary interference and multi-path fading. The degree of effectiveness of a particular frequency-hopping system for a given transmission channel depends on several factors such as the hopping rate, the size of the hops, and the number of data symbols transmitted during each hop interval [Tor85], [RW90], [Gib93]. If the number of data symbols transmitted during a hop interval is less than or equal to unity, the process is called fast frequency-hopping. If the number of data symbols transmitted during a hop interval is greater than one, the process is called slow frequency-hopping. As several symbols are transmitted per hop interval in slow frequency-hopping, an impairment at a given hop frequency will result in a burst error (i.e., several data bits are lost). This means that some 
form of error correction coding is required for the transmitted data. In fast frequencyhopping, a high level of data redundancy is present because there can be several hops per transmitted symbol. If some hops are not properly received due to interference or fading at a given frequency, there is still enough information available to make use of diversity combining techniques to make a correct symbol decision. The number of hops taken per symbol interval, L, is known as the "order" of diversity [BF86] and is given by;

$$
L=\frac{T_{\text {symbol }}}{T_{\text {hop }}}
$$

Where $\mathrm{T}_{\text {symb: }}$ is the data symbol duration, and $\mathrm{T}_{\text {hop }}$ is the hop duration. Note that the above equation is only valid under the assumption that the hops are sufficiently separated in frequency to provide the receiver with $\mathrm{L}$ resolvable transmission paths per data symbol.

Rather than reducing the effective energy of interfering signals as in direct-sequence spread-spectrum systems, frequency-hopping systems achieve their real advantage through interference avoidance.

Two main problems encountered in PLL based frequency-hopping are spectral-splatter, and transient mismatch between the transmit and receive synthesizers [AG89]. During a hop transient, shown in Figure (2-15), a simple frequency-hroping system has no control over the transmitted frequency spectrum, and many undesired frequency components are present in the output of the transmitter. 
Figure 2-15: PLL Transient Response to Frequency hop

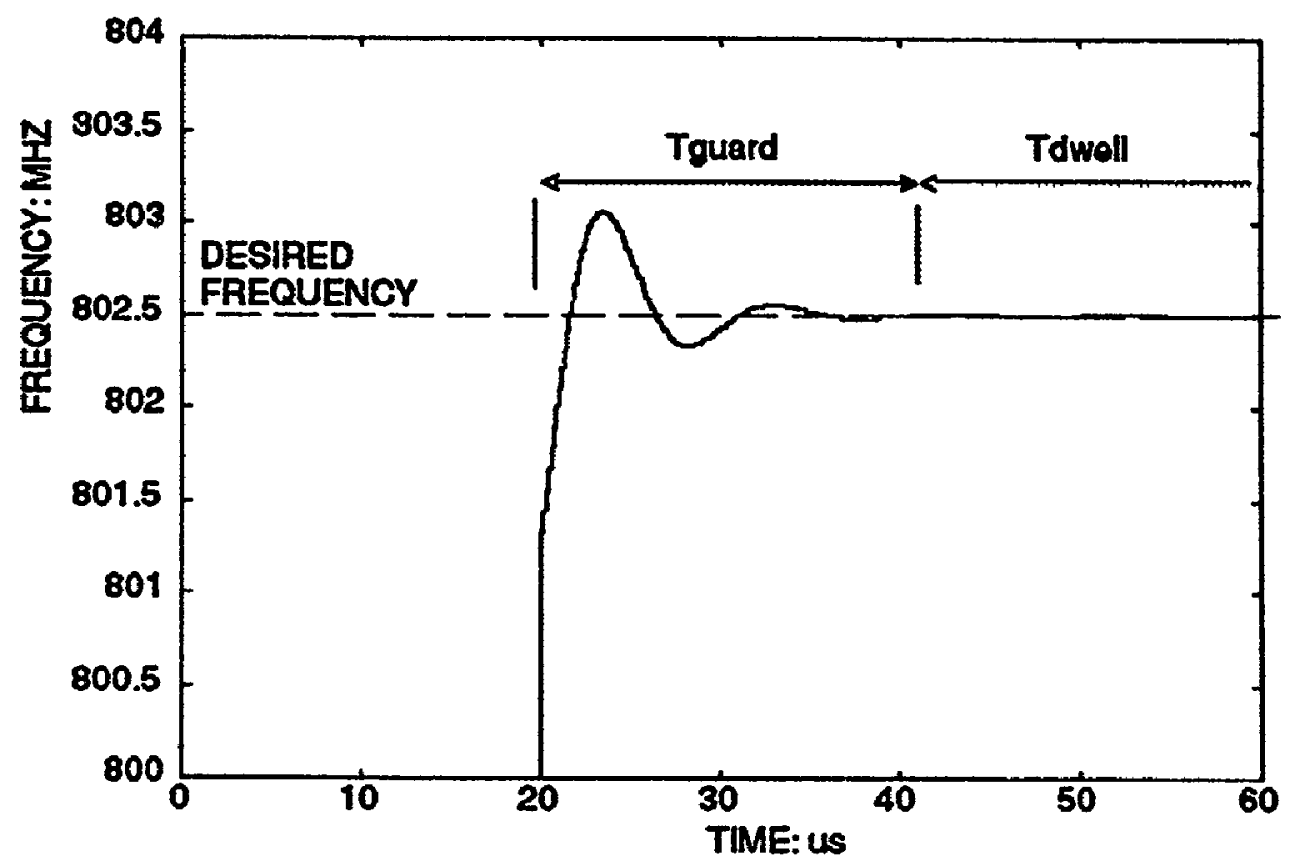

This phenomena is known as spectral-splatter and results in transmitter energy loss during each hop, as well as adjacent channel interference. Additionally, if there is a mismatch in the hopping transient of the receive synthesizer from that of the transmit synthesizer, bursts of frequency-error occur in the receiver IF at the hopping rate, producing an overall degradation in the receiver SNR. Some common solutions used to reduce spectral-splatter and the effects of synthesizer mismatch are voltage-controlled oscillator (VCO) pre-tuning [AG89], ping-ponging multiple synthesizers [AG89], and employing hop interval dwell and guard times [MLT91]. These methods are briefly reviewed in the following paragraphs.

VCO pre-tuning makes use of a digital-to-analog (D/A) converter and a summing block placed in both the transmitter and the receiver synthesizer PLL between the loop filter and the VCO. When a hop occurs, the VCO control voltage is changed directly, as well as the normal loop frequency control, in a coordinated manner, reducing the effects of transient mismatch in the system. As the D/A and summing blocks are outside of the loop, the output frequency of the synthesizer can be changed very rapidly, and the loop catches up 
afterwards, removing any residual error.

A frequency-hopping system that ping-pongs two synthesizers in both the transmitter and the receiver can be designed. When one frequency is being transmitted, the second synthesizer is pre-set to the next frequency. To transmit the next frequency in the hopping sequence the two synthesizers are exchanged (ping-ponged), eliminating the effects of PLL transient error and increasing the potential hopping rate, but not allowing phase coherence.

Transient guard and dwell times are often employed in frequency-hopping systems to avoid the effects of IF error and were shown in Figure (2-15). During a frequency hop, the IF signal is not sampled at the receiver until the loop transients have settled out. Since the IF contains no frequency-error during the sample (dwell) time, the received data is not corrunted. In some systesis, the transmitter output power is turned off during the guard times in order to reduce the spectral-splatter produced by the hop transients. For fast frequency-hopping, transient guard and dwell times are no longer useful as there is more than one transient step present per transmitted symbol.

The above sclutions, though effective, lead to higher system complexity and cost, or a reduction in effective data sampling time that increases the receiver bit-error rate (BER).

An alternative method of generating a frequency-hopped carrier is to use direct-digital frequency-synthesis as outlined in the frequency-synthesis section of this chapter. The only transient response present during a frequency change is that of the filter placed after the output of the D/A converter. The designer has a large degree of flexibility in the selection of this filter. The maximum output frequency for direct digital-synthesis is limited to less than one half the clock frequency, making it necessary to add a frequency upconversion stage for most RF applications. This makes DDS less attractive for frequency-hopping systems.

In frequency-hopping CDMA, each user in the system follows a different hopping pattern. If the patterns used are orthogonal, each user will only receive the transmission meant for 
them. However, in practice it is impossible to find a large number of perfectly orthogonal codes, and collisions between users do occur. The effect of such collisions can be greatiy reduced through the use of error correction codes [VMS84]. If perfectly orthogonal hopping codes are employed, frequency-hopping CDMA can be viewed as a form of timevarying frequency-division multiple-access. That is, for a given instant in time, each user is assigned a particular frequency slo! in which to transmit their data.

Timing specifications for hopping code synchronization between transmitters and receivers in frequency-hopping systems are usually not as stringent as those required for directsequence spread-spectrum. Code phase synchronization between the transmitter and receiver is required within a fraction of the hopping period, which is much larger than the inverse of the spreading bandwidth. In a direct-sequence system with the same bandwidth, synchronization is required within a fraction of the code bit period, which is equal to the inverse of the spreading bandwidth.

\subsubsection{Broadband-coded FM}

In conventional FM systems, a carrie is frequency modulated by a time-varying signal that can be eithe: analog or digital in nature (the latter is known as frequency shift-keying). The two basic categories of FM transmission are narrowband FM, and broadband FM. For the ease of this discussion, the modulating signal is chosen to be sinusoidal with a modulation rate and frequency deviation as defined in Figure (2-16). 
Figure 2-16: Modulating Signal for Sinusoidal FM

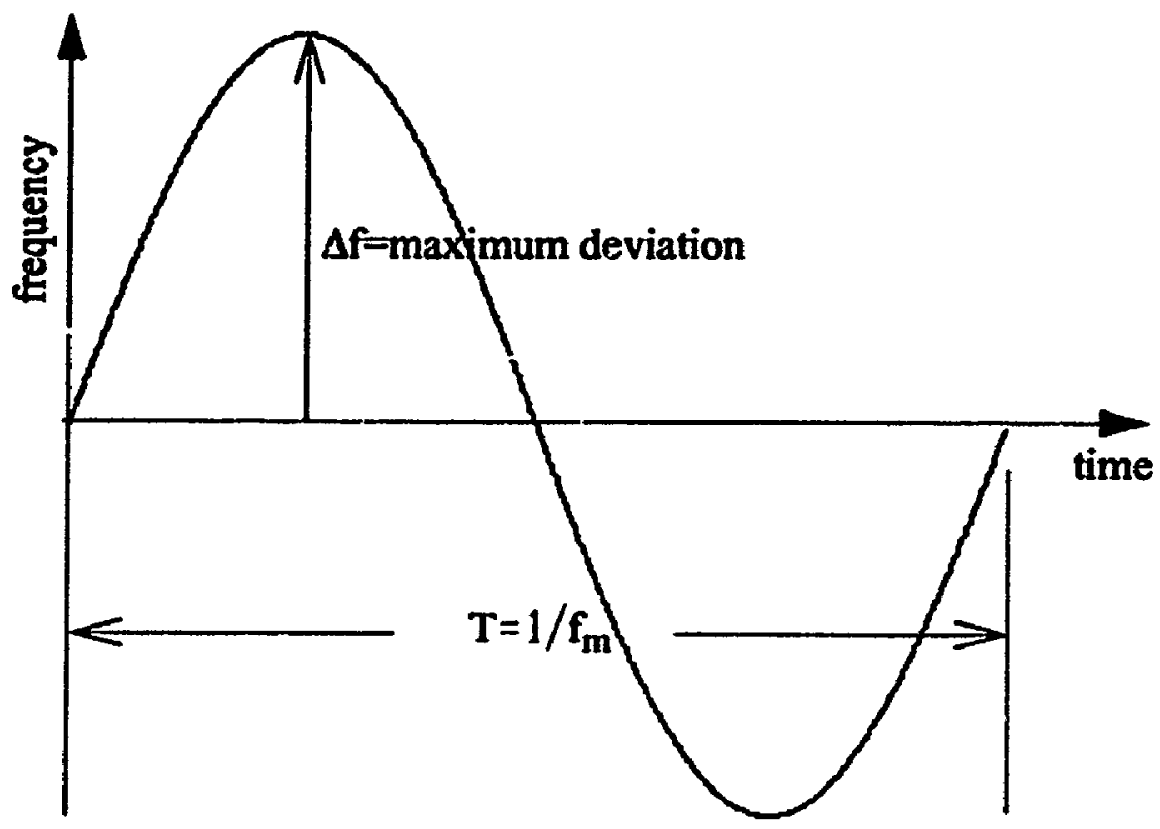

The resulting spectrum for the FM modulated signal is composed of two components, the frequency content of the modulating signal as described by the modulation rate, and the maximum instantaneous frequency deviation as described by the amplitude of the modulating signal. The modulation index of the signal, $\beta$, is defined as;

$$
\beta=\frac{\Delta \omega}{\omega_{m}}
$$

Where $\Lambda .0$ is the maximum instantaneous frequency deviation of the modulating signal, and $\omega_{\mathfrak{m}}$ is the modulation rate. If the modulation index is much less than one, the transmitted signal is called narrowband FM and the transmission bandwidth is approximately equal to $2 \omega_{m}$ [Rod91]. If the modulation index is much greater than one, the transmitted signal is called broadband FM and the narrowband approximation can no longer be used. In this case, the transmission bandwidth is approximately equal to $2 \Delta \omega$. The frequency spectrum of a broadband FM modulated carrier is actually described by a series of Bessel functions 
occurring at the modulation rate and that span the full frequency deviation of the signal. For a complete mathematical analysis of both narrowband and broadband FM refer to [Rod91]. The above rest as are summarized by Carson's rule for the bandwidth occupancy of an FM modulated signal, $\mathrm{W}$, given by;

$$
W \cong 2\left(\Delta \omega+\omega_{m}\right)
$$

Broadband FM is not commonly viewed as being a form of spread-spectrum transmission because the modulating signal is the transmitted data. However, if broadband modulation of the carrier is performed separately from the data modulation using a pseudo-random waveform known by both the transmitter and the receiver, and fiequency-coherent detection is used to despread the signal in the receiver, broadband-coded FM fits into the category of spread-spectrum. In recent work performed in our laboratory, broadband-coded FM was used as the transmission method for a demonstration radio system used in a performance study of various novel radio components and architectures [Ken94]. 


\section{Chapter 3}

\section{Interpolated Frequency-hopping}

\subsection{Introduction}

Interpolated frequency-hopping, a subset of broadband-coded FM introduced in this chapter, provides an alternative solution to the problems encountered in simple frequencyhopping, replacing analog complexity with digital complexity, which is a good trade-off for VLSI. A block diagram of a possible implementation of an interpolated frequency-hopping system is shown in Figure (3-!).

The differences between the system presented and the standard frequency-hopping system that was shown in Figure (2-13) are the inclusion of a digital filter placed between the hepping code generator and the synthesizer control word input, differences in the signalprocessing is he performed on the output of the discriminator, and in the assumption that the frequency synthesizer can be accurately controlled using a digital input.

In this system, the hopping code generator generates a series of numbers that represent the desired output frequencies, which are interpolated by the digital filter and sent to the synthesizer control input. An example of the output signal (fiequency versus time) for an interpolated frequency-hopping transmitter is shown in Figure (3-2). The interpolation filter for this example uses full response signalling techriques and a Gaussian pulse shape. 
Figure 3-1: An Interpolated Frequency-hopping Spread-spectrum System

\section{Transmitter}

FM modulated input data

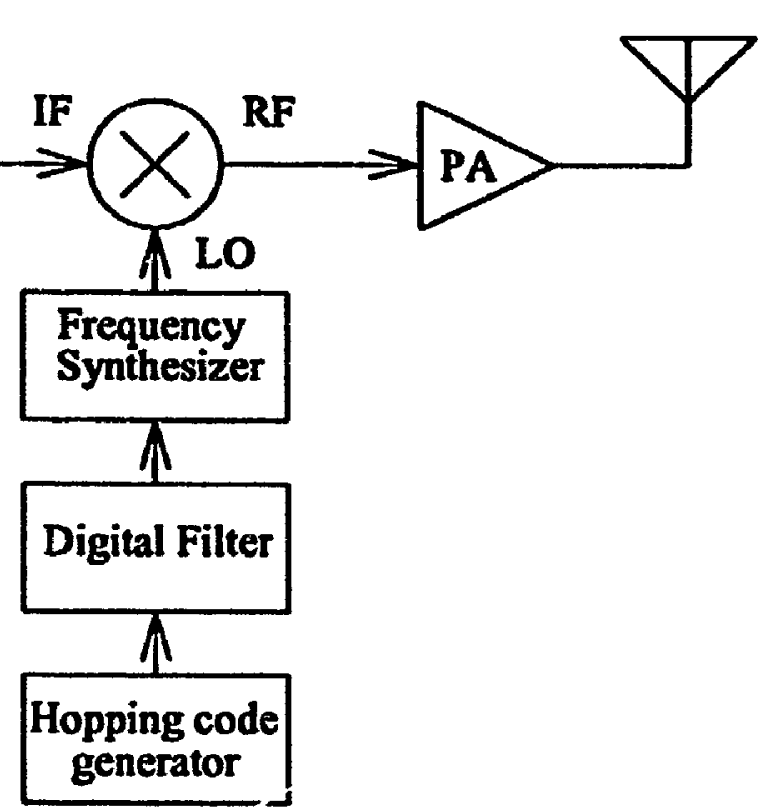

Receiver

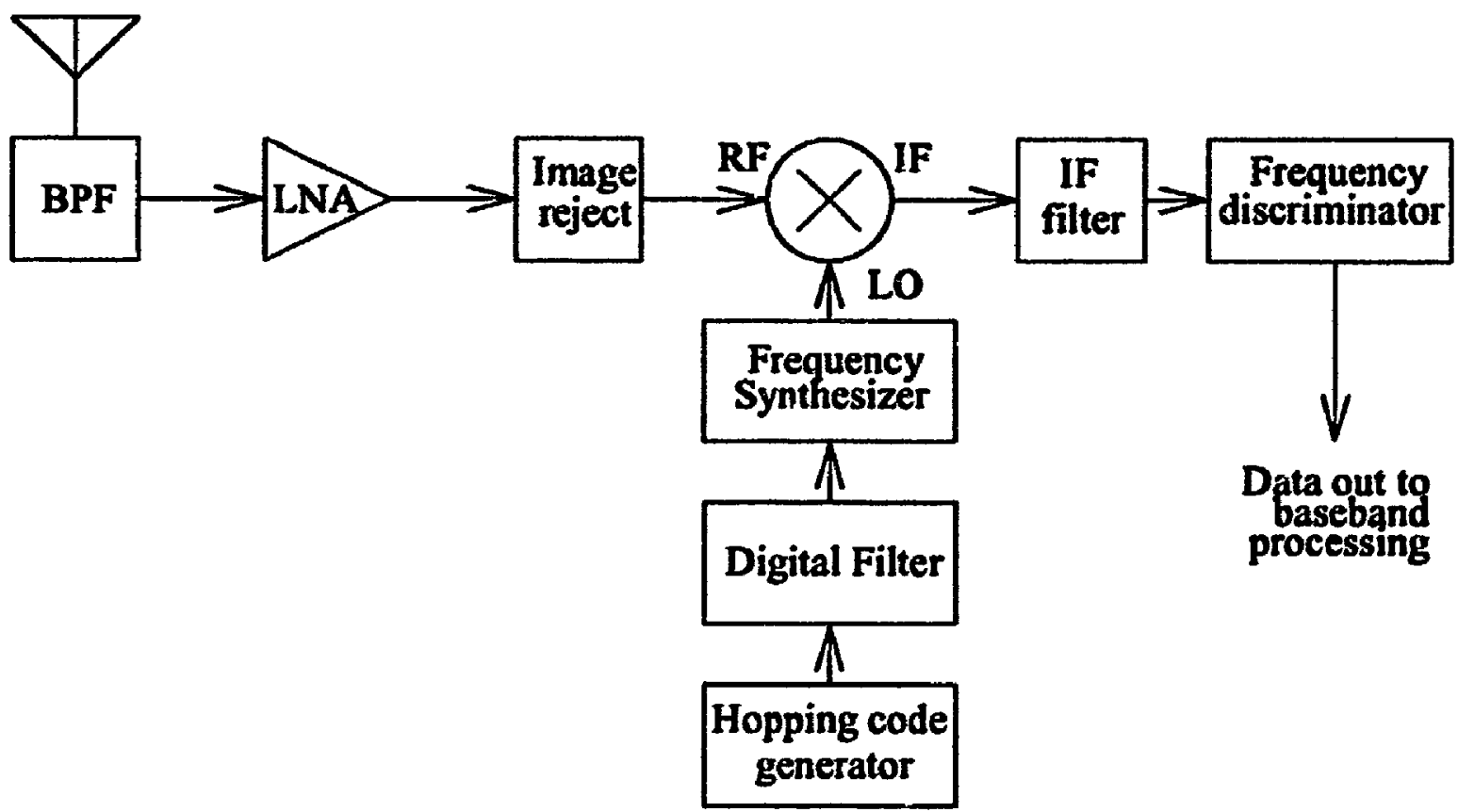


Figure 3-2: An Interpolated Frequency-hopping Spread-spectrum Signal

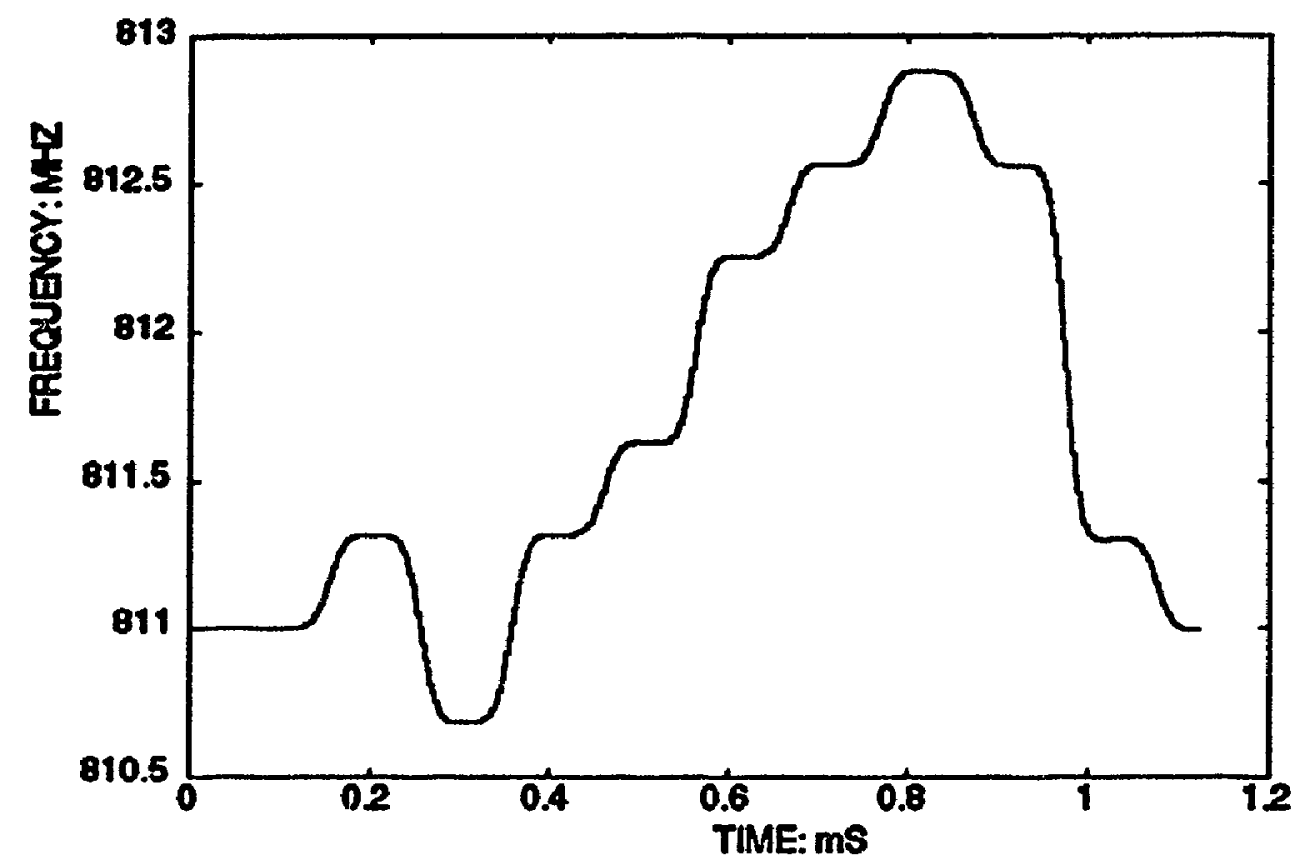

\subsection{IF Distortion Due to PLL Mismatch}

A closer look at the transient-error mechanism in a PLL based frequency synthesizer will help to define interpolated frequency-hopping. Figure (2-5) shows the block diagram of a fractional-N PLL frequency synthesizer. The output frequency of the loop is controlled by changing $N$, the average division ratio of the divider, which consists of both an integer and a fractional part [MC90]. Although the divider control input is digital, for the purposes of simplicity and convention, the dynamics of the PLL are modelled as a continuous time system. This simplification is reasonable as long as the PLL closed-loop bandwidth is much smaller than the sampling rate of the underlying discrete process [Craw94]. Assuming that $f_{\text {in }}(s)$ is the normalized instantaneous frequency input to the synthesizer, the instantaneous frequency output (i.e., the Laplace transform of instantaneous frequency), $f_{\text {out }}(s)$, is;

$$
f_{o u t}(s)=H_{c l}(s) f_{i n}(s)=f_{r e f} H_{c l}(s)(n+\delta(s))
$$


Where $\mathrm{H}_{\mathrm{cl}}(\mathrm{s})$ is the closed-loop transfer function of the PLL from the digital control input to the VCO output, $f_{\text {ref }}$ is the reference frequency, $n$ is the integer portion of the average divider division ratio, and $\delta(s)$ is the continuous time equivalent of the digital fiequency control word applied to the synthesizer input.

For the frequency-hopping transceiver previously shown in Figure (2-13), if $f_{\text {in }}(s)$ is the hopping code, and no data is applied to the modulation input of the system, then for the transmitter;

$$
f_{R F}(s)=H_{c l T x}(s) f_{i n}(s)+f_{I F}
$$

and for the receiver,

$$
f_{L O}(s)=H_{c l R x}(s) f_{i n}(s)
$$

Where $H_{c l T x}(s)$ and $H_{c l R x}(s)$ are the transmitter and receiver PLL closed-loop transfer functions respectively, and $f_{\mathrm{IF}}$ is the IF frequency.

In the receiver, assuming perfect code phase synchronization, the IF output in the absence of data modulation is given by;

$$
I F(s)=f_{R F}(s)-f_{L O}(s)=f_{i n}(s)\left[H_{c I T x}(s)-H_{c I R x}(s)\right]+f_{I F} 3-4
$$

Where the closed-loop transfer functions of the synthesizers, $\mathrm{H}_{c}(\mathrm{~s})$, are related to their open-loop transfer functions, $\mathrm{H}_{\mathrm{ol}}(\mathrm{s})$, by; 


$$
H_{c l}(s)=\frac{H_{o l}(s)}{1+H_{o l}(s)}
$$

Then, substituting (3-5) into (3-4), the IF output becomes;

$$
I F(s)=f_{i n}(s)\left[\frac{H_{o l T_{x}}(s)}{1+H_{o l T x}(s)}-\frac{H_{o l R x}(s)}{1+H_{o l R x}(s)}\right]+f_{I F}
$$

Where IF(s) is the instantaneous IF output signal, and $H_{\text {olTx }}(s)$ and $H_{o I R x}(s)$ are the transmitter and receiver PLL open-loov transfer functions respectively. The instantaneous frequency-error present in the IF output is given by;

$$
I F_{\text {error }}(s)=f_{\text {in }}(s)\left[\frac{H_{o l T x}(s)}{1+H_{o l T x}(s)}-\frac{H_{o l R x}(s)}{1+H_{o l R x}(s)}\right]
$$

The above equation shows that if the open-loop transfer functions of the two synthesizers are identical, or if the open-loop gains are infinite, the IF frequency error will be zero. The effects of PLL mismatch are smaller for input frequencies that are much lower than the PLL corner-frequency and increase as the input appiouaches the corner frequency (this will be shown more clearly in the design section of this thesis). We will now assume that modulated data has been linearly added (in freouency) to the transmitted signal, as shown in Figure (3-2), and comes through linearly in the absence of channel degradations and other sources of noise and distortion, to the input of the frequency discriminator of Figure (3-2). Assuming the discriminator characteristic is perfectly linear, Equation (3-7) represents a degradation of the received baseband signal introduced by incomplete despreading of the pseudo-random hopping sequence, $f_{\text {in }}(s)$, due to synthesizer mismatch. The best case SNR of the received baseband signal is then given by; 


$$
S N R=\frac{k_{d}^{2} \int_{0^{+}}^{2 \pi B H}\left[\left|f_{d a t a}(j \omega)\right|\right]^{2} d \omega}{k_{d}^{2} \int_{0^{+}}^{2 \pi B W}\left[\left(f_{i n}(j \omega) \mid\right)^{2}\left(\left|\frac{H_{o I T x}(j \omega)}{1+H_{o l T x}(j \omega)}-\frac{H_{o l R x}(j \omega)}{1+H_{o l R x}(j \omega)}\right|\right)^{2}\right] d \omega}
$$

Where $f_{\text {data }}(j \omega)$ is the transmitted data, $K_{d}$ is the discriminator characteristic in Volts/Hz, and $\mathrm{BW}$ is the frequency bandwicth of interest. By filtering the hopping code prior to the synthesizer input, it is possible to reduse the high frequency components of the transmitted signal and thus to minimize the PLL mismatch error.

Assuming that the interpolation filter, being digital, is identical in both the transmitter and receiver, and following the same derivation as before, the IF frequency error for interpolated frequency-hopping is given by;

$$
I F_{\text {error }}(s)=f_{i n}(s) F(s)\left[\frac{H_{o l T x}(s)}{1+H_{o l T x}(s)}-\frac{H_{o l R x}(s)}{1+H_{o l R x}(s)}\right]
$$

Where $F(s)$ is the continuous time equivalent transfer function of the digital interpolation filter. Using the same assumptions as for frequency-hopping and including the interpolation filter, the SNR is given by;

$$
\begin{aligned}
& \left.k_{d}^{2} \int^{2 \pi B W} \because{ }_{d a t a}(j \omega) \mid\right]^{2} d \omega \\
& S N R=\frac{0_{d}^{2 \pi} \int_{0^{+}}^{2 \pi B W}\left[\left(\left|f_{i n}(j \omega)\right|\right)^{2}(|F(j \omega)|)^{2}\left(\left|\frac{H_{o l T x}(j \omega)}{1+H_{o l T x}(j \omega)}-\frac{H_{o l R x}(j \omega)}{1+H_{o l R x}(j \omega)}\right|\right)^{2}\right] d \omega}{3-10}
\end{aligned}
$$

The end result of filtering the hopping code prior to the synthesizer control input is the 
effective lowpass filtering of baseband receiver distortion generated by PLL mismatch, increasing the received SNR. If a perfectly linear system is assumed, the order of placement of the different filters used in the system should have no effect on the final result. However, it is important to note that if the interpolation filter were placed in the receiver rather than the transmitter, the reduction in spectral-splatter could no longer be achieved, and unwanted filtering of the received data would occur. The degenerate case of interpolated frequency-hopping is standard frequency-hopping where $F(j \omega)=1$, reducing Equation (310) to Equation (3-8) given previously. In interpolated frequency-hopping the carrier signal moves continuously and smoothly in frequency, and there are no bursts of frequency-error present in the IF output at the code rate. The PLL synthesizer must continuously track the input frequency, resulting in low levels of frequency-error being translated to the IF output. Provided that the frequency-error is small enough to maintain an adequate SNR in the receiver, valid data is available at all times, improving performance if the receiver makes use of the whole symbol period in its processing. The smooth carrier movement may also help to reduce the complexity and improve the performance of the hardware required to synchronize the received and locally generated codes. This potential simplification exists because code phase information is available to the receiver at all times rather than only during the hop intervals as in standard frequency-hopping.

As indicated in Equation (3-10), the frequency-error present during interpolated frequencyhopping transmission can be minimized by using greater levels of code interpolation, by increasing the loop bandwidth of the synthesizers, and by carefully matching the loop filters. Using large frequency deviations for the FM data modulation will also help to increase the baseband SNR.

\subsection{Frequency Spectrum}

It is useful to derive an analytical expression for the power spectral density of an interpolated frequency-hopping signal. This will be done using the auto-correlation method outlined in [AS83] and [LW90]. The following derivation is performed assuming that no data modulation is present on the carrier in order to simplify the resulting equations. In the 
time domain, an interpolated frequency-hopping signal, $S(t)$, can he expressed as;

$$
S(t, \beta)=\sqrt{2 \mathrm{P}} \cos \left[2 \pi f_{c} t+\theta_{c}(t, \beta)\right]
$$

Where $P$ is the transmitted power, $f_{c}$ is the carrier centre frequency, and $\theta_{c}$ is the instantaneous carrier phase which is a function of both time and the hopping code $\beta$. The hopping code consists of a series of numbers that represent the magnitudes of the desired frequency steps and is denoted by;

$$
\beta=\left\{\beta_{0}, \beta_{1}, \beta_{2}, \beta_{3} \ldots \beta_{N-1}\right\}
$$

Where $\mathbf{N}$ is the number of steps in the hopping sequence. The code phase is given by;

$$
\theta_{c}(t, \beta)=2 \pi \sum_{n=0}^{N-1} \beta_{n} T_{h} C\left(t-n T_{h}\right)
$$

Where $T_{h}$ is the hop period, and $C(t)$ is the phase response and is given by;

$$
C(t)=\int_{-\infty}^{i} g(\tau) d \tau
$$

Where $g(t)$ is the frequency pulse given by;

$$
g(t)=\frac{1}{c} \sum_{i=0}^{m} k_{i} b\left(t-i T_{h}\right)
$$


Where $k_{i}$ are the coefficients of the system polynomial for the partial response filter (see background section), $c$ is a normalization factor, and $b(t)$ is the shape chosen for the baseband pulse. The spectral properties of the transmitted interpolated frequency-hopping signal are controlled through the choice of the PRS system polynomial, and the baseband pulse shape, $b(t)$. Interpolated frequency-hopping is broadband frequency modulation of a carrier, and therefore, auto-correlation methods can be used to obtain the transmitted power spectral density function. The normalized complex lowpass equivalent signal, $u(t, \beta)$, is given by;

$$
u(t, \beta)=\exp \left(j\left[\theta_{c}(t, \beta)\right]\right)
$$

The complex lowpass auto-correlation function is given by;

$$
R_{u}(t, t+\tau)=E\left[u^{*}(t, \beta) u(t+\tau, \beta)\right]
$$

Where * denotes the complex conjugate. This equation can be re-written as;

$$
R_{u}(t, t+\tau)=E\left\{\exp \left[j\left(\theta_{c}(t+\tau, \beta)-\theta_{c}(t, \beta)\right)\right]\right\}
$$

$R_{u}(t, t+\tau)$ is periodic in time with a period of $\mathrm{NT}_{\mathrm{b}}$. As there is no specific time origin, we will assume that the origin is uniformly distributed over $\left[0, \mathrm{NT}_{\mathrm{h}}\right]$. Invoking cyclostationary methods [LW90], the average auto-correlation is given by; 


$$
R_{u}(\tau)=\frac{1}{N T_{h}} \int_{0}^{N T_{h}} R_{u}(t, t+\tau) d t
$$

The power spectral density of the equivalent lowpass interpolated frequency-hopping signal is then given by the Fourier transform of the average auto-correlation given by;

$$
S_{u}()=\int_{0}^{N T_{k}} R_{u}(\tau) \exp (-j 2 \pi f \tau) d \tau
$$

Numerical methods can be used along with Equations (3-11) to (3-20) to obtain plots of the theoretical power spectral density for the interpolated frequency-hopping signal.

\subsection{Diversity}

Interpolated frequency-hopping should have a diversity order which is at least as high as that obtained through the use of a comparable frequency-hopping system. Therefore, Equation (2-17) can be used to obtain a rough estimate of the diversity order that can be achieved for a given interpolated frequency-hopping system. It should be noted that for interpolated frequency-hopping, because the carrier is continuously moving in frequency, it is difficult to define a specific order of diversity as the transmission can no longer be discretely separated into resolvable paths. 


\section{Chapter 4}

\section{Proposed System}

\subsection{An Interpolated Frequency-hopping Transceiver}

A proposed architecture for a radio frequency (RF) transceiver that employs interpolated frequency-hopping transmission is shown in Figure (4-1). The block diagram is similar to a typical frequency modulation (FM) system with some additional components included for code generation and filtering, as well as phase synchronization of the received and locally generated codes. The transmitted carrier signal is FSK modulated. The data is filtered prior to addition to the interpolated frequency-hopping carrier in order to improve its spectral characteristics. It has been shown that the use of bandwidth efficient data modulation improves the performance of a frequency-hopping system [LW90].

On the receiver side, the received signal is down-converted to an intermediate frequency by mixing it with an exact copy of the transmitted code produced by the Rx synthesizer. In order to obtain the full benefit of interpolated frequency-hopping, it is necessary to process the IF signal over the whole symbol period, implying a high sampling rate or continuous time integration. Á frequency discriminator has recently been demonstrated [Ken94], [BC94], [BC93], which can accept an FM signal at a relatively high IF frequency and produce a one-bit frequency to digital conversion at high oversampling rates through the use of $\Delta-\Sigma$ techniques [CT91]. The discriminator is well suited for data signals that contain large frequency deviations such as might be used in interpolated frequency-hopping, and is indicated as a block in Figure (4-1). However, if the transmitter were used in a CDMA system, it would be necessary to keep the data deviations relatively small in order to prevent adjacent channel interference. This means that the data deviations should be chosen carefully in order to balance these two requirements. 
Figure 4-1: An Interpolated Frequency-hopping Spread-spectrum Transceiver System

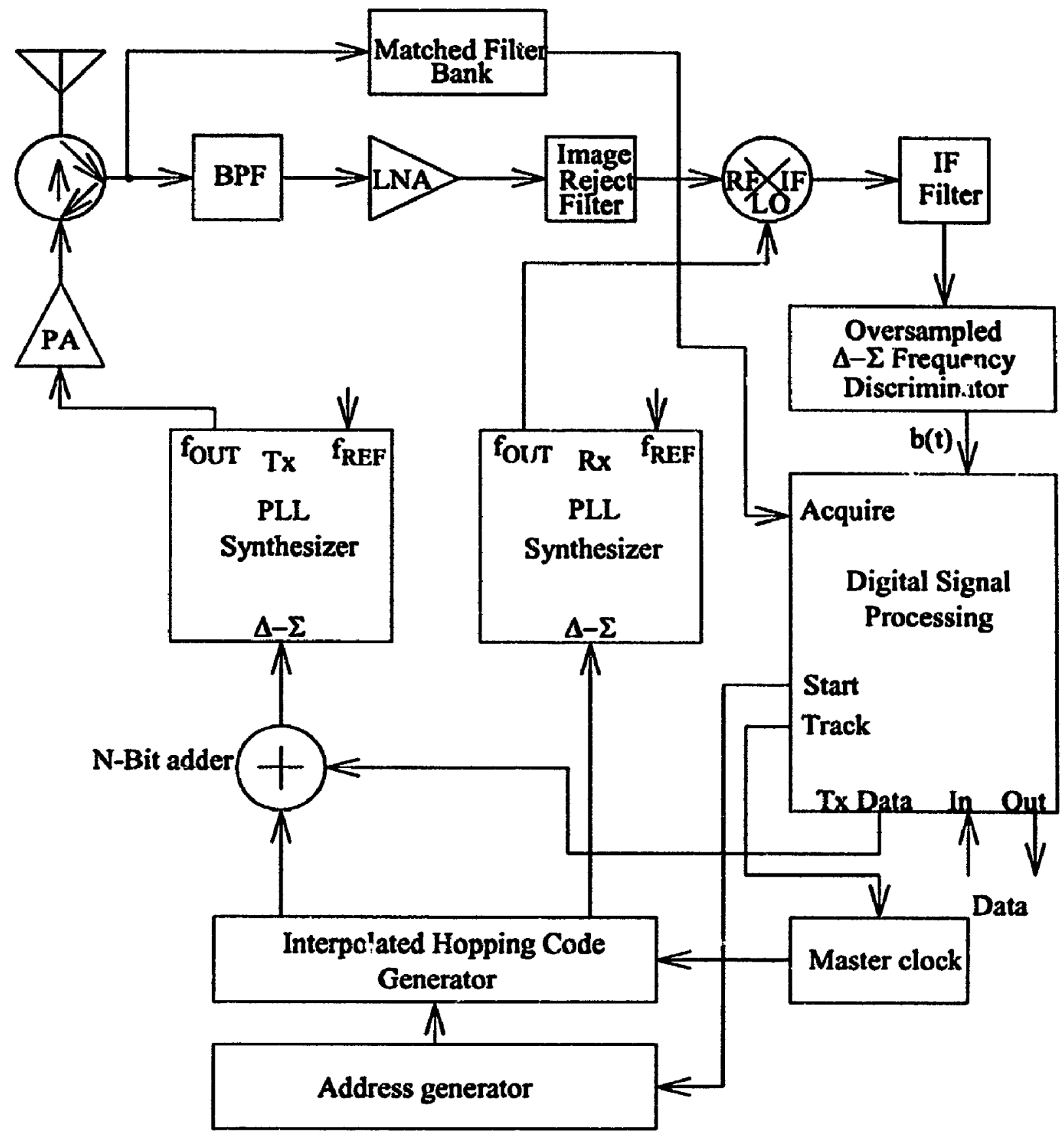

A fully integrated $\Delta-\Sigma$ frequency discriminator has been tested [BC93], and printed circuit board implementations exist [Ken94]. The output one-bit digital bitstream, $b(t)$, has an average density of ones which represents the 'irequency deviations of the input signal. This represents the baseband data as well as an error signal which is directly proporiional to the 
frequency difference between the received and local hopping codes. The digital-signalprocessing block of the transceiver is used to extract the error signal and obtain a measure of the phase difference between the received and local hopping codes, and to decimate the output of the discriminator. The error signal is then used as feedback to control the phase of the hopping code generator clock for fine-time code tracking. One possible method for initial acquisition of the interpolated frequency-hopping signal is to place a short bank of matched filters in paraliel with the input signal path, as shown in Figure (4-1). Each filter is matched to a particular hop frequency. The combined outputs of the filters produce a correlation peak when a short sequence of hops, part of the larger sequence transmitted, is detected. (Note that due to practical issues, in an actual implementation of the transceiver, the received signal would have to be downconverted before passing it through the matched filters). The resulting signal is used by the DSP block to set the local code generator to the starting ROM address. This method of coarse synchronization provides the system with the fast acquisition times that are required in mobile radio applications. Since in the transmitter the data clock is derived from the interpolation filter clock, once code synchronization is achieved in the receiver, data timing recovery is not required.

The frequency synthesizer used in both the transmitter and receiver side of Figure (4-1) is a fractional-N PLL synthesizer similar in construction to the one shown in Figure (2-5), with the addition of a digital $\Delta-\Sigma$ modulator. The input to the modulator is a K-bit wide digital control word which represents the desired instantaneous frequency output of the synthesizer. The modulator converts this into a binary bitstream whose average density of ones represents the required division ratio. High frequency resolution, and low phase noise levels are obtained through oversampling, the noise shaping properties of the $\Delta-\Sigma$ modulator, [CT91], and additional filtering by the PLL.

The receive synthesizer in Figure (4-1) would be modulated with the interpolated frequency-hopping code, at the LO frequency. The transmit synthesizer in Figure (4-1) would be modulated with the interpolated frequency-hopping code as well as the transmitted data, at the RF frequency. It would be possible in an alternate realization of the transceiver to share a single synthesizer for both the $\mathrm{Tx}$ and $\mathrm{Rx}$ function, modulated only 
with the interpolated frequency-hopping code. A mixer would then be required in the Tx path. An experimental demonstration of the transmitter path has recently been presented [RC94], for Gaussian minimum-shift-keying (GMSK) signals, which gives an efficient embodiment of the digital interpolation filter assumed for the hopping code generator and is now described. This implernentation of the filter makes use of $\Delta-\Sigma$ quantized tap coefficients and is shown in Figure(4-2).

Figure 4-2: Digital Interpolation Filter

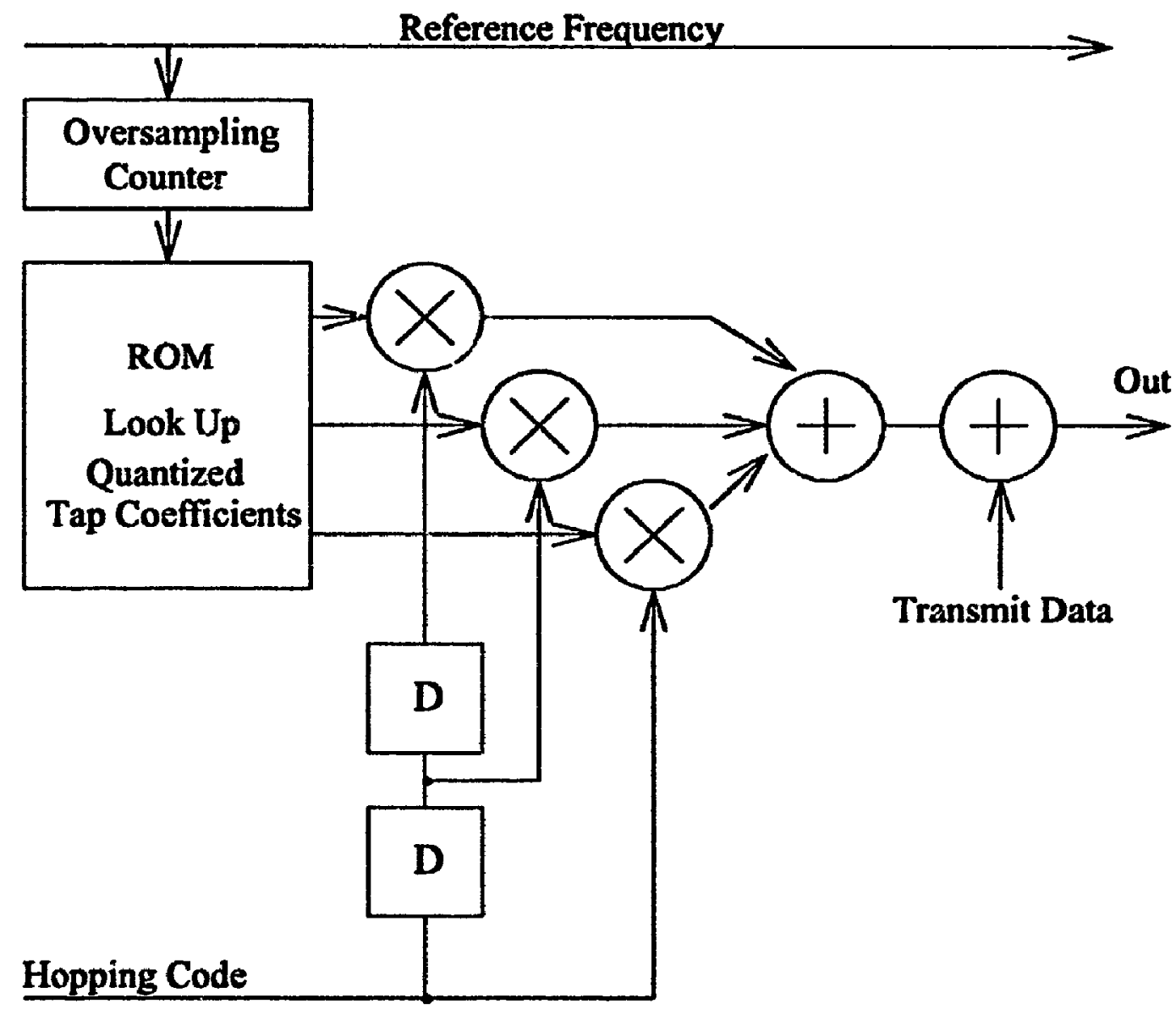

In this example, the desired filter has a Gaussian lowpass response, and makes use of partial response signaling techniques in order to combine the effects of three hops in its processing. By quantizing the tap coefficients using software $\Delta-\Sigma$ methods prior to storing their values in a ROM, the required multiplications are reduced to single bit operations, greatly simplifying the required hardware. If the hops are all kept identical in size, the 
hopping code generator needs only to store "1s" and " $0 \mathrm{~s}$ ", indicating the polarity of the required frequency step. The output of the digital filter is fiist scaled, and then fed into a nbit digital accumulator that keeps track of the hops taken by the carrier. The output of the accumulator is passed through an $n$-bit digital $\Delta-\Sigma$ modulator which produces an oversampled serial bitstream that is used to control the fractional- $\mathrm{N}$ frequency synthesizer.

If the transceiver were employed in a system with multiple users, it might be possible to use phase-division multiple-access (PDMA) rather than CDMA. In PDMA, each user in the system follows the same interpolated hopping code, but is assigned a different phase offset within the sequence. The base station would keep transmitting all of the possible carrier phases whether data were being transmitted or not. Initial acquisition by a handset would be achieved by locking on to an arbitrary base station signal. The handset would then identify itself to the base station, which would return the code-phase information required for that particular user. A PDMA system would require only a single hopping code, and would allow several users to simultaneously transmit over the same frequency bandwidth. 


\section{Chapter 5}

\section{System Design}

\subsection{Introduction}

This chapter outlines the design of the interpolated frequency-hopping system that was tested during the course of this thesis. A description of the overall transmission testbed is given, followed by design details for the hopping-code generator and interpolation filter, the white noise source and bit-error counter, and the FSK discriminator.

\subsection{Transmission Testbed}

The experimental laboratory setup used to test interpolated frequency-hopping transmission is shown in Figure (5-1). In order to make the test system versatile, and to avoid as many hardware implementation issues as possible, hopping code generation, code interpolation, data generation, and $\Delta-\Sigma$ quantization are performed in non-real time using software running on a personal computer. The resulting data files are then down-loaded to the bit generator board shown, and fed to the system in real time. The bit generator board has two independent channels each of which is capable of storing 1 million bits and sending them out to the system repeatedly at a rate of $10 \mathrm{MHz}$. The fractional-N synthesizers (Tx and $R x$ ) shown are controlled by the single bit bitstreams that are noise shaped using $\Delta-\Sigma$ techniques and are stored in the bit generator board [Ken94], [RCK93]. Note that $f_{\text {in }}(s)$ as defined in Equation (3-1) now contains quantization noise as well as the hopping sequence, and data if required. Synchronization of the transmitter and receiver is achieved through the use of interconnecting cable links, and the transmission channel is modelled by the signal path shown within the dashed lines of Figure (5-1). 
Figure 5-1: Experimental Setup

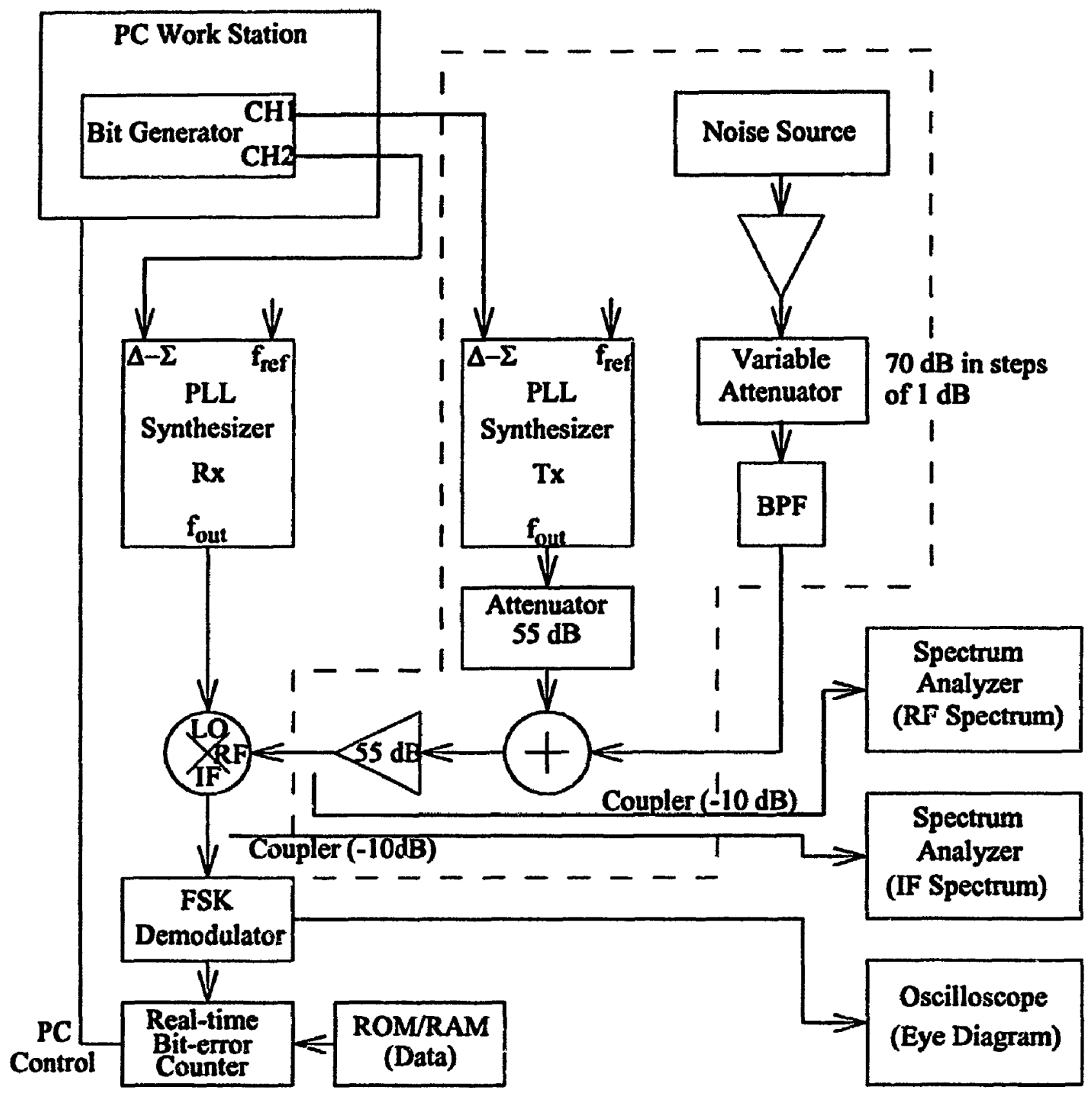

The fractional-N synthesizers were implemented at the printed circuit board level and incorporated with a modulus extension circuit allowing division ratios between 80 and 85 to be used, for a reference frequency of $10 \mathrm{MHz}$ [Ken94]. The centre frequencies of the 
transmitter and receiver synthesizers were chosen to be $811 \mathrm{MHz}$ and $809 \mathrm{MHz}$ respectively, with a spreading bandwidth of $5 \mathrm{MHz}$. This allowed an IF frequency of 2 MHz to be chosen in order to take advantage of the narrow resolution bandwidth of the $\mathbf{4 0}$ MHz bandwidth spectrum analyzer (HP3585A), although the implemented transceiver would have a much higher IF frequency in order to resolve problems such as image rejection.

The output signal of the transmitter synthesizer is attenuated by approximately $55 \mathrm{~dB}$, at which point white Gaussian noise is added through the power combiner shown. In order to prevent the subsequent saturation of the various other components in the system by noise, the noise produced by the generator is passed through a bandpass filter (BPF) that limits its bandwidth to $20 \mathrm{MHz}$ centered around the RF frequency. In order to facilitate bit-error rate testing, a variable attenuator is placed between the amplifier and BPF in order to allow the noise level to be attenuated by up to $70 \mathrm{~dB}$ in steps of $1 \mathrm{~dB}$. The signal, along with the added noise, is then passed through an amplifier which provides approximately $55 \mathrm{~dB}$ of gain, amplifying both the signal and the noise. The signal is then mixed with the local oscillator output in order to despread it and downconvert it to the $2 \mathrm{MHz}$ IF frequency employed by the receiver. The output of the mixer is fed into a FSK demodulator that consists of an IF filter followed by a phase-shift type frequency discriminator. The discriminator output is integrated over the data symbol period using an integrate and dump filter, the output of which is fed into a latched comparator that decides whether a one or a zero was transmitted. The received data bits are then compared to a stored version of the transmitted cata in realtime using the bit-error rate (BER) counter board shown in Figure (5-1). By varying the level of the noise generated by the source shown in Figure (5-1) using the step attenuator, it is possible to obtain the bit-error rate of the transmission system for a given noise level. The transmitted $\mathrm{Eb} / \mathrm{No}$ at an attenuator setting of zero was then measured using a spectrum analyzer, and used as an absolute reference point in subsequent measurements. In this way, plots of bit-error rate vs. Eb/No were obtained for the system. 
Although the experimental setup does have certain limitations due to the limited storage capability and maximum clock rate of the bit generator card, it allows different forms of transmission to be tested easily and quickly by changing data files, rather than reconfiguring the hardware.

\subsection{Hopping Code and Interpolation Filter}

For the purposes of the testing performed in this thesis the hopping code used did not have to be optimal, and CDMA issues were not considered. The code was created by generating an array of desired output frequencies using MATLAB [MAT92]. The array is a superposition of two different waveforms. The first waveform is a fixed array of uniform, randomly distributed frequency points with maximum and minimum frequency deviations as shown in Figure (5-2 a). The maximum frequency deviation between any two points in this array defines the maximum hop size. The second array defines a triangular-wave with the same number of points as the uniform array, and maximum and minimum frequency deviations as shown in Figure (5-2 b). The two arrays were added together and the resulting waveform is shown in Figure (5-2 c).

Figure 5-2: Hopping Code (Frequency vs. Time)

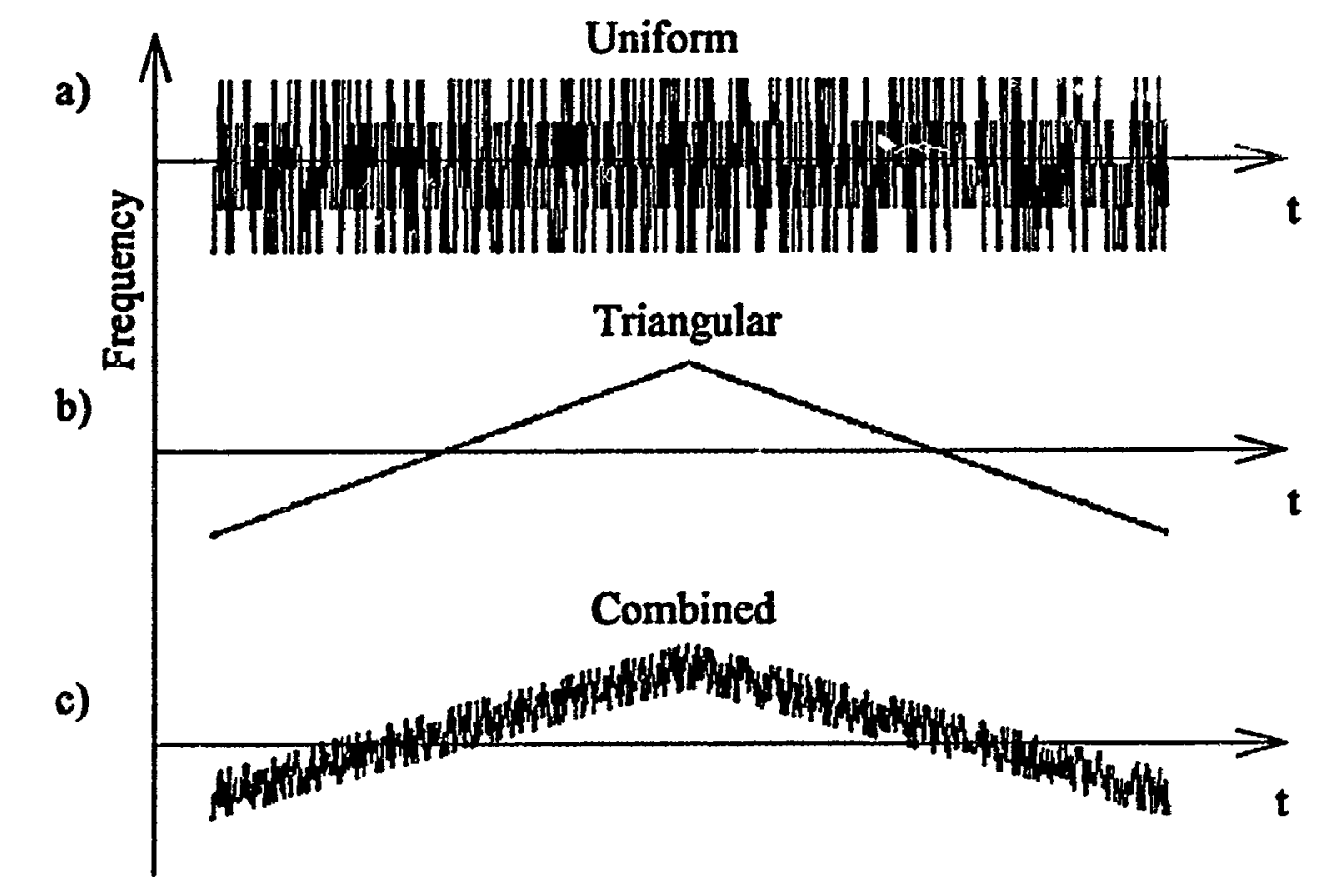


As the hop sizes defined in the first array become larger, the frequency deviation of the triangular waveform in the second array must be made smaller, as the total available hopping bandwidth is limited to a certain size.

This hopping cide provides the system with the random nature and quick frequency changes required for spread-spectrum transmission, while providing an underlying uniform frequency distribution that slowly moves the carrier over the entire spreading bandwidth.

The purpose of the code interpolation filter is to reduce the bandwidth of the pseudorandom code in order to minimize the transient phase-error generated in the PLL during frequency transitions. The hopping code used in this thesis has a uniform frequency distribution over time, and the resulting frequency spectrum takes on a sinx/x form, with nulls occurring at integer multiples of the fundamental hopping rate as shown by the simulation results presented in Figure (5-3) for a code rate of $10 \mathrm{KHz}$. There are also discrete spectral components occurring at regular frequency spacings equal to the inverse of the code period, although they are not visible on the plot. The purpose of the filter is to limit the energy of the code to the main lobe, and to minimize energy at frequencies greater than the fundamental hopping rate. This results in smoother frequency transitions and a reduction in spectral-splatter. 
Figure 5-3: Simulated Frequency Spectrum of Hopping Code

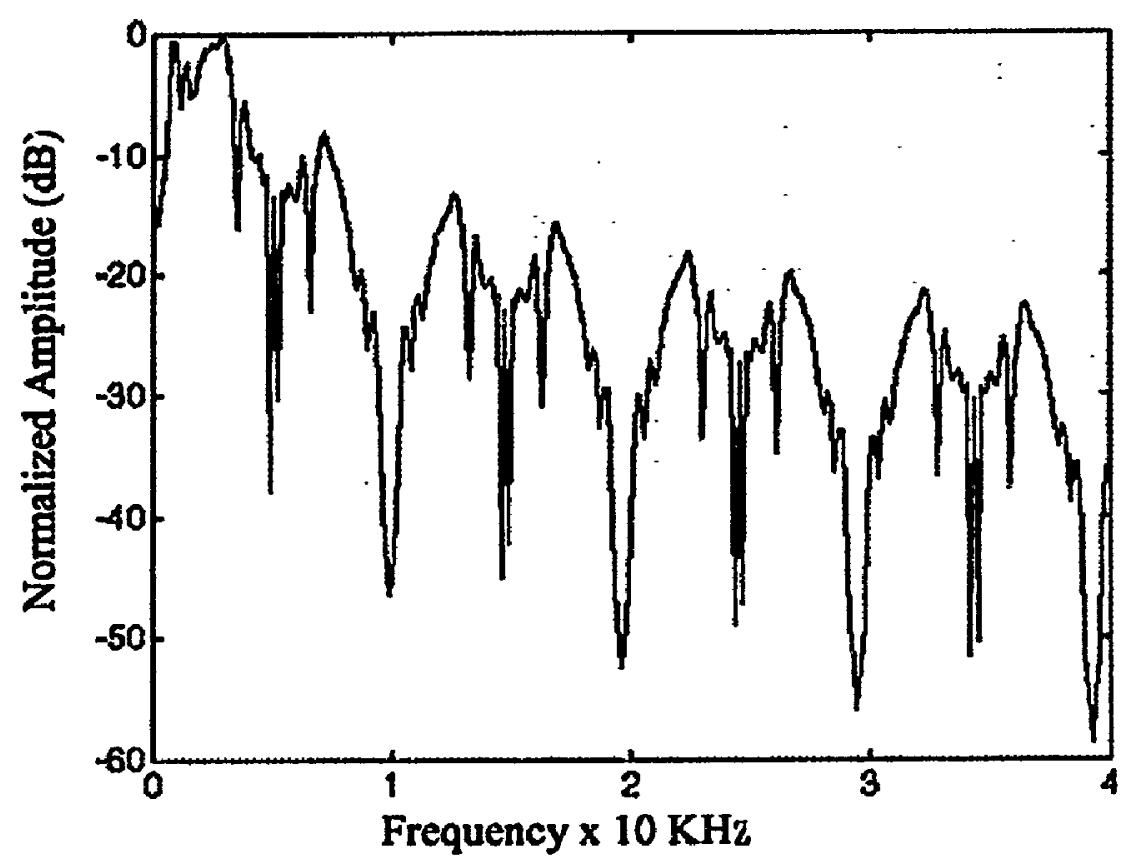

The digital filter chosen for the interpolated frequency-hopping system designed in this thesis is a PRS filter as described in the background section, with a system polynomial, $F(D)$, equal to;

$$
F(D)=1+D+D^{2}
$$

and a Gaussian baseband pulse truncated symmetrically to three symbol periods. The bitstream generator can only store $2^{20}$ bits and therefore there is a fixed relationship between the hopping rate and the number of taps used in the interpolation filter. The number of taps, $L$, is equal to;

$$
L=3\left(\frac{2^{20}}{2^{N}}\right)
$$


Where $2^{N}$ is the number of hops taken over the entire code repetition period. The $3 \mathrm{~dB}$ bandwidth of the filter, $B$, is given by;

$$
B=\frac{f_{s}}{L}
$$

Where $f_{s}$ is the sampling rate. The hopping rate is given by;

$$
H=\frac{f_{s}\left(2^{N}\right)}{2^{20}}
$$

If Equations (5-2), (5-3), and (5-4) are combined, we find that the $3 \mathrm{~dB}$ bandwidth of the Gaussian lowpass filter is always equal to one-third of the hopping rate. Figure (5-4) shows the simulated magnitude transfer function for the interpolation filter.

Figure 5-4: Magnitude Response of Digital Interpolation Filter

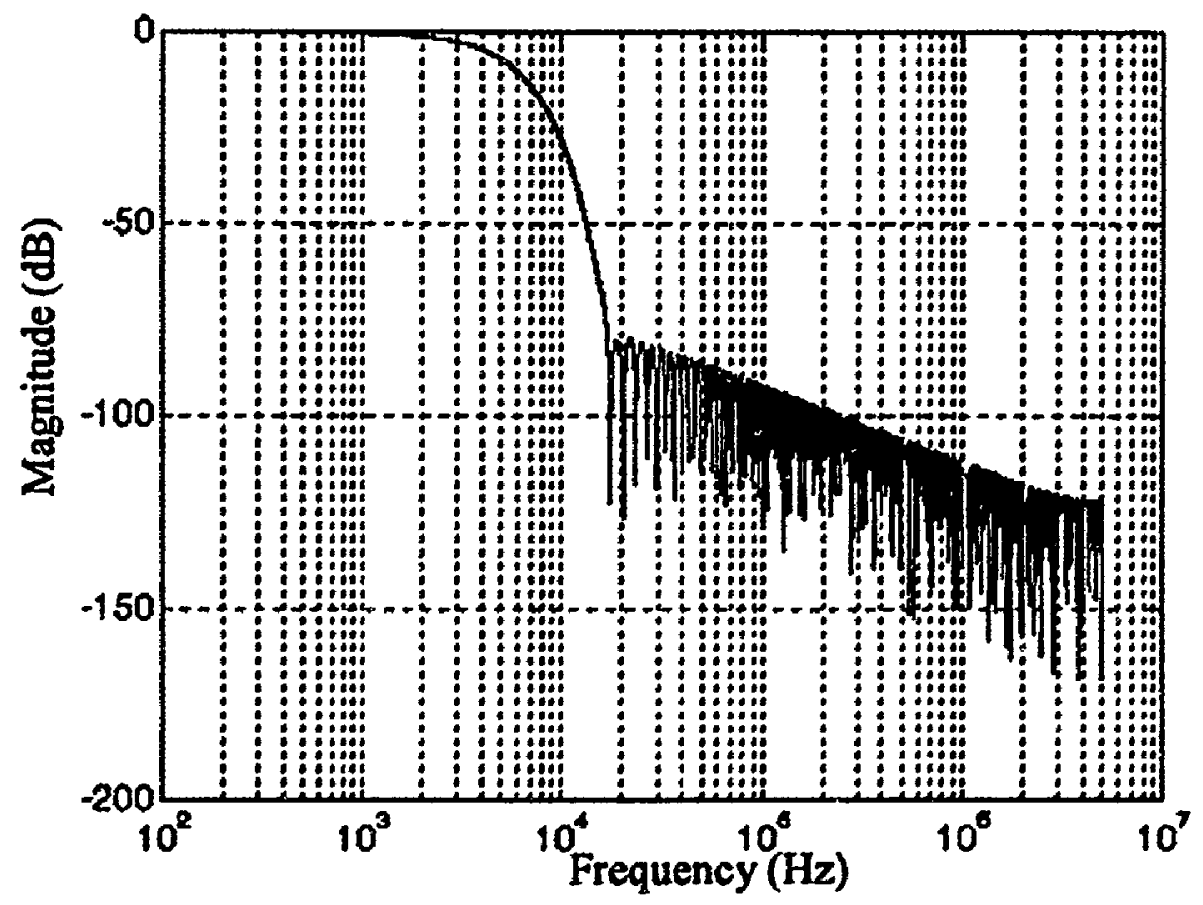


It can be seen that at the position of the second lobe of the code spectrum shown in Figure (5-3), the lowpass filter shown in Figure (5-4) provides approximately $75 \mathrm{~dB}$ of attenuation. Figure (5-5) shows the simulated spectrum of the hopping code after it has been filtered, and by comparison to Figure (5-3) it can be seen that the sidelobe energy has been greatly reduced. For hopping rates that fall well within the closed-loop bandwidth of the PLL, the improvement obtained through the filtering of the hopping code is not very high. However, as the fundamental hopping rate approaches, or exceeds the loop bandwidth, the code sidelobes extend well past the corner-frequency of the loop and a great improvement in system performance can be obtained through interpolation of the code. This is true because the degree of mismatch between the two synthesizers increases with frequency, as the loop gain decreases with frequency. The approximate mismatch transfer function for the synthesizers used in the laboratory was obtained assuming a 10\% component mismatch between the loop filters. A plot of the simulated mismatch magnitude transfer function is presented in Figure (5-6).

Figure 5-5: Frequency Spectrum of Filtered Hopping Code

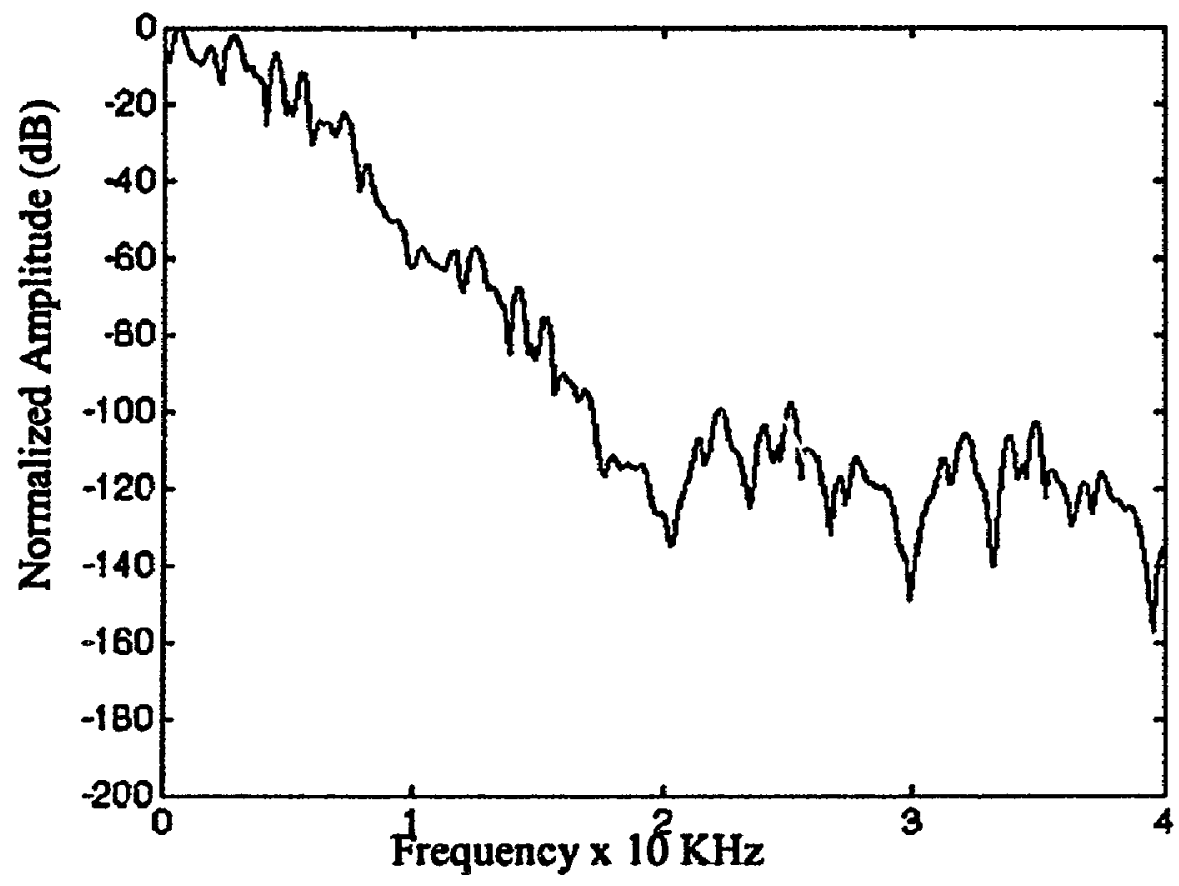


Figure 5-6: Magnitude Response of Synthesizer Mismatch Function

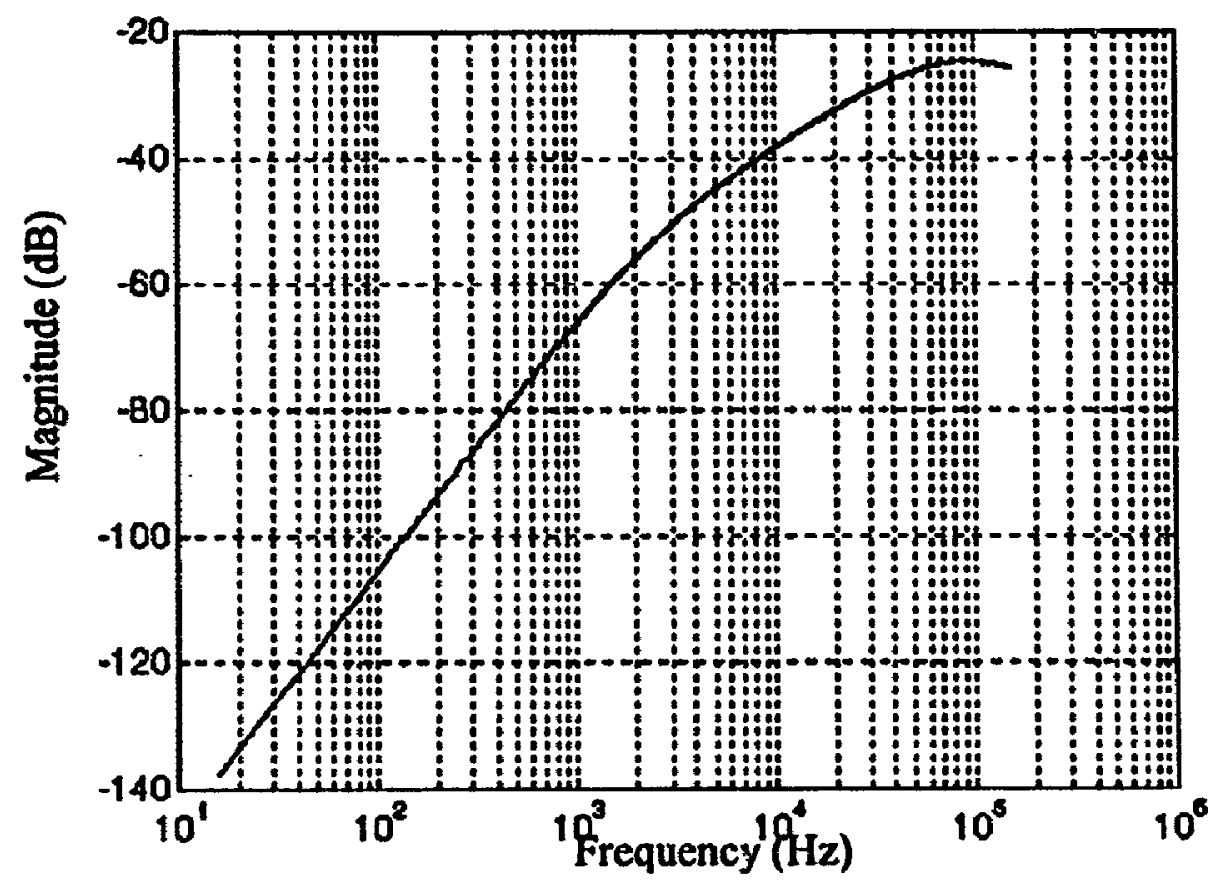

The above statements are supported by both simulation and laboratory measurements performed in this thesis. The values required for the filter tap coefficients were generated using MATLAB, and stored in a disk-file as double-precision floating point numbers.

The files containing the hopping code array and the filter tap coefficient values were used by a function written in $\mathrm{C}$ to perform the code filtering and accumulation of the hopping frequencies. This function is called by a larger $C$ program called ROSAD, which generates the $\Delta-\Sigma$ quantized control files that are downloaded to the bit generator board [Ril91].

\subsection{White Noise Source and BER Board}

In order to perform bit-error rate testing on the radio system designed for this thesis, it was necessary to build both a white noise source and a real-time bit-error counter.

Figure (5-7) shows the circuit diagram for the white noise generator that was built for the test system. The noise source used in the circuit is a Noise/Com NC513 drop in noise module. The NC513 generates flat noise, $(+/-2.0 \mathrm{~dB}$ from $0.2-2000 \mathrm{MHz})$, with a Gaussian distribution and an excess-noise-ratio (ENR) of $51 \mathrm{~dB}$. ENR is a measure of the excess 
noise generated by a source with respect to the thermal noise generated by a $50 \mathrm{ohm}$ load at $290 \mathrm{~K}$. The output power spectral density for the noise source is given by;

$$
P=-174 \mathrm{dBm} / \mathrm{Hz}+\mathrm{ENR}=-174+51=-123 \mathrm{dBm} / \mathrm{Hz}
$$

The amplifiers shown in Figure (5-7) are Mini-circuits MAR-8 monolithic RF amplifiers, and provide approximately $38 \mathrm{~dB}$ of gain to the output of the noise generator circuit.

\section{Figure 5-7: White Noise Source}

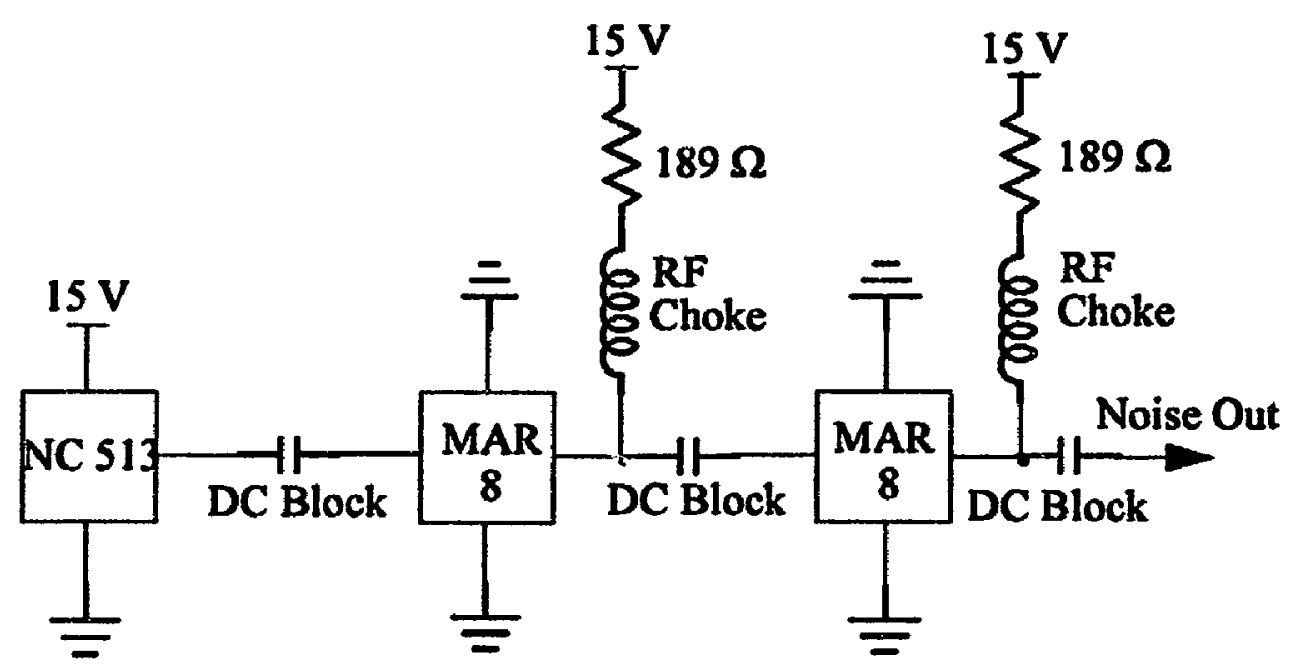

Figure (5-8) shows a block diagram of the real-time bit-error counter ${ }^{1}$ that was designed and built for this thesis. Full schematics of the BER board are provided in Appendix A. The board is a fully digital design, and is controlled via a parallel port by a program running on a PC type computer. The board is designed to function from a $10 \mathrm{MHz}$ clock, and accepts an input data rate of $78 \mathrm{~Kb} / \mathrm{s}$. The board operates as follov/s. An exclusive OR gate is used 
to compare the demodulated data from the FSK board to a copy of the transmitted data bits stored either in a RAM or a ROM on the BER board. If a received bit is different from the stored version (i.e., a bit-error has occurred), the error counter is incremented by one. The board is capable of counting up to 65,536 bit errors, at which point a count overflow indicator is triggered. The board also keeps track of the number of bits compared in order to allow the bit-error rate to be calculated. The different counter settings possible are summarized in Table (5-1). The board allows independent phase adjustment of the stored data and the sampling clock, with a time resolution of $100 \mathrm{~ns}$. This allows the received data to be properly aligned with the stored data. The BER board also generates a pulse which is used by the FSK demodulator board to control the transmission gate that dumps the contents of the integrate and dump filter at the end of each symbol period, and to latch the output of the comparator that follows the integrator. 
Figure 5-8: Real-time Bit-error Counter

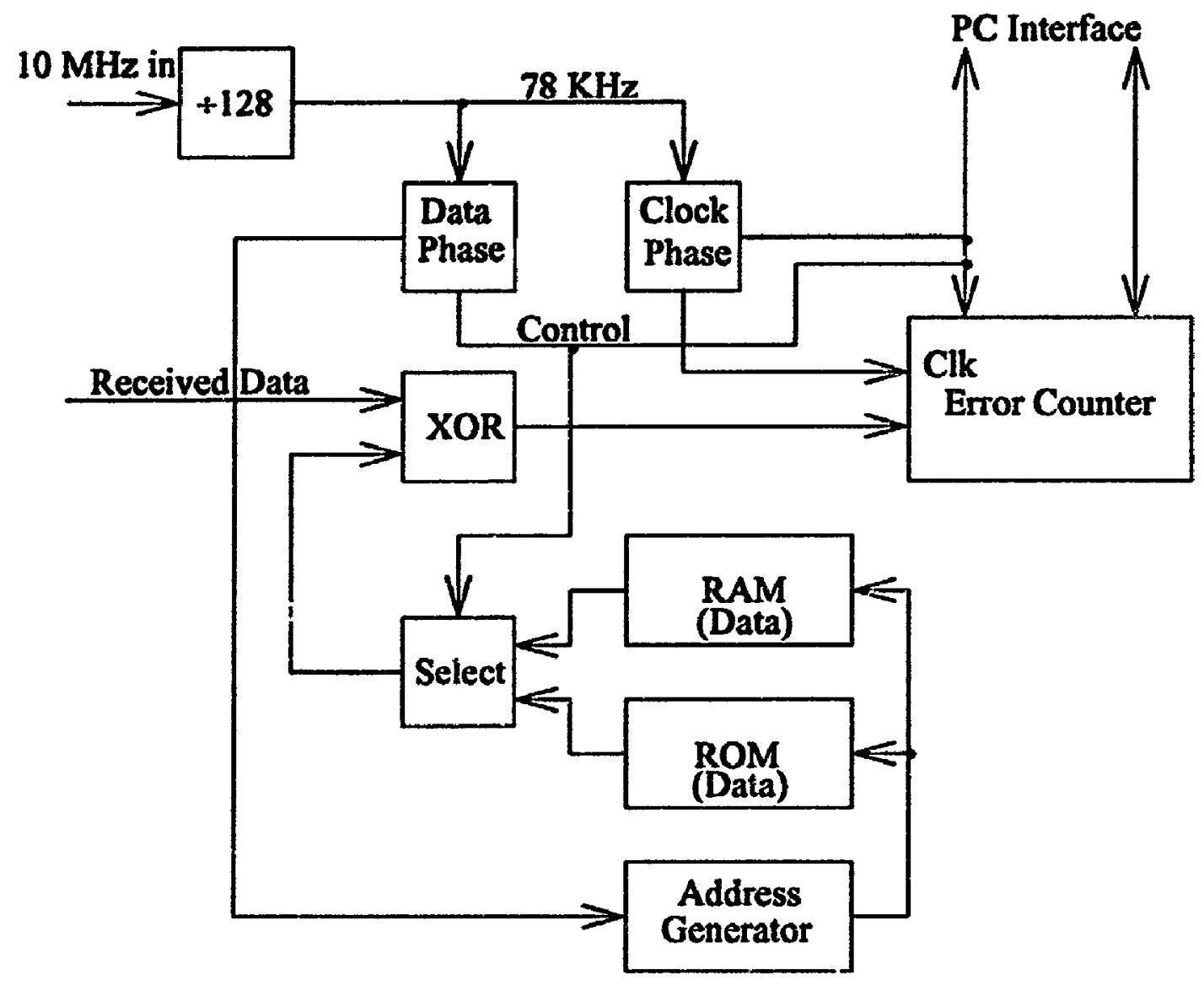

Table 5-1: BER Board Settings

\begin{tabular}{|l||l|}
\hline BIT COUNT & \multicolumn{1}{c|}{ TIME } \\
\hline \hline 1000 & $12.8 \mathrm{~ms}$ \\
\hline 10,000 & $128 \mathrm{~ms}$ \\
\hline \hline 100,000 & $1.28 \mathrm{~s}$ \\
\hline \hline 1 Million & $12.8 \mathrm{~s}$ \\
\hline 10 Million & $2.1 \mathrm{~min}$ \\
\hline 100 Million & $21.3 \mathrm{~min}$ \\
\hline \hline 1 Billion & $3.6 \mathrm{hrs}$ \\
\hline
\end{tabular}




\subsection{Analog Frequency Discriminator}

In order to obtain the best performance possible for symbol detection in the receiver for the purposes of bit-error rate testing, the FM discriminator output must be followed by a filter that is matched as closely as possible to the pulse shape used for the transmitted data symbols [Stre79]. For the purpose of simplifying the testing performed in this thesis, the only puise shaping applied to the transmitted symbols was that imposed by the transient response of the PLL frequency synthesizers used. It is assumed that the transient response of the PLL results in transmitted symbols that are approximately boxcar shaped, making the matched filter required in the receiver a simple integrate and dump over the symbol period. This assumption provided significant hardware simplifications in the design of the FSK demodulator board. A block diagram of the ideal receiver is shown in Figure (5-9), along with a diagram of the receiver structure used in this thesis. The matched filter is followed by a decision circuit.

A block diagram of the FSK demodulator board is shown in Figure (5-10). The $2 \mathrm{MHz}$ IF signal is first passed through an active filter in order to remove unwanted high frequency components from the input spectrum. The lowpass filter is followed by an amplitude limiter whose purpose is to remove residual AM from the received signal. The signal is then passed through an LC resonant tank circuit which shifts it in phase. The phase shifted version of the signal is then phase compared to the unshifted version using an exclusive OR (XOR) logic gate. The output of the XOR gate is a pulsewidth modulated bitstream whose average value is a representation of the original data. The datastream is then lowpass filtered in order to reduce carrier feedthrough, and passed through an integrate and dump filter whose output is fed to a latched comparator which provides TTL level data bits that can be sent to the BER board for real-time bit-error counting. The following paragraphs describe the different sections of the demodulator board in greater detail, and provide circuit schematics. Full schematics of the demodulator board are provided in Appendix B. 
Figure 5-9: Ideal and Non-ideal FSK Receiver Structure

Ideal Receiver

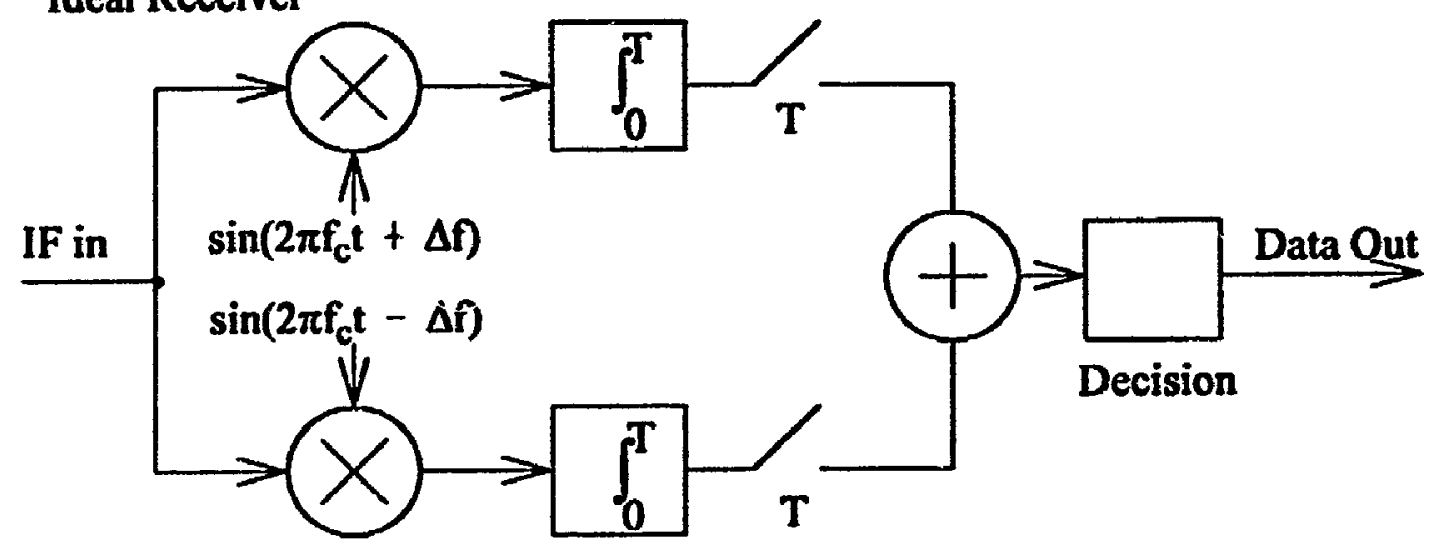

Non-ideal Receiver

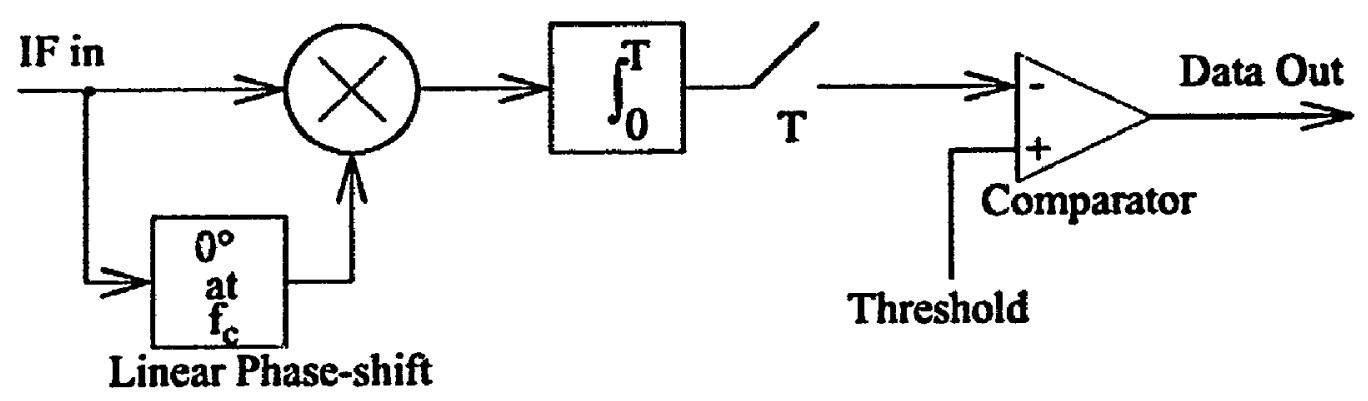

Figure 5-10: Block Diagram of FSK Demodulator Board

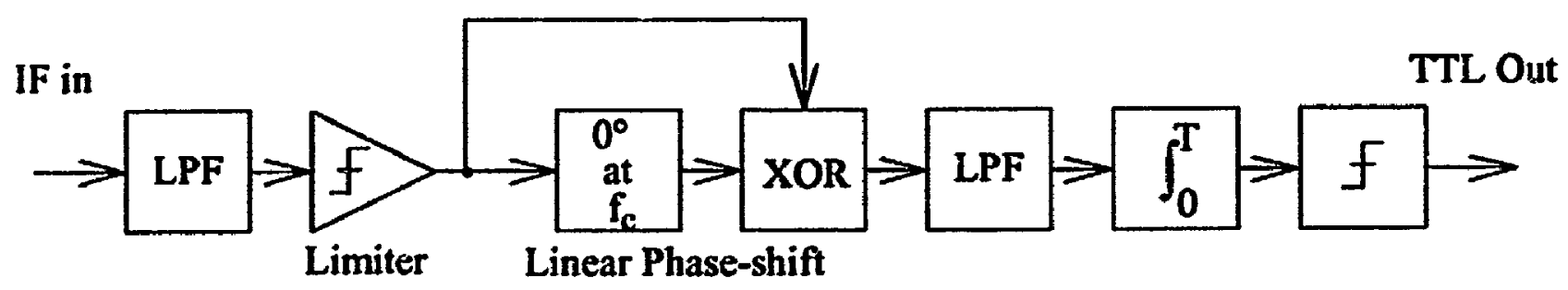




\subsubsection{IF Filter}

The active If filter used on the demodulator board is a Tow-Thomas biquad and is shown in Figure (5-11).

Figure 5-11: Tow-Thomas Biquad

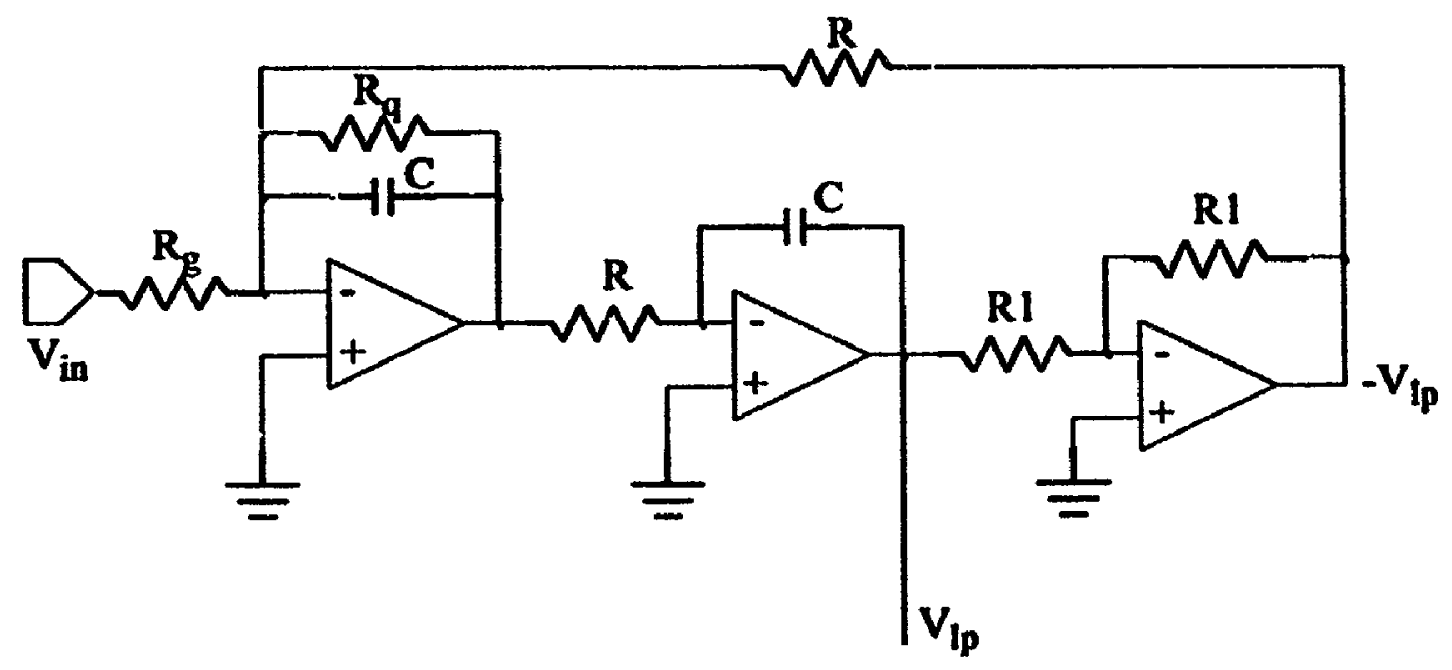

This filter structure implements a second-order transfer function that can either be a lowpass, bandpass, or highpass response depending on which output point is selected by the designer.

The transfer function for the lowpass response is given by;

$$
\frac{V_{L P}(s)}{V_{i}(s)}=\frac{\left(\frac{R}{R_{g}}\right) \frac{1}{R^{2} C^{2}}}{s^{2}+s \frac{1}{R_{q} C}+\frac{1}{R^{2} C^{2}}}
$$

Where the corner frequency, $\omega_{0}$, is given by; 


$$
\omega_{o}=\frac{1}{R C}
$$

and the $\mathrm{DC}$ gain, $\mathrm{H}_{0}$, is given by;

$$
H_{0}=\frac{R}{R_{g}}
$$

and the $Q$ of the filter is given by;

$$
Q=\frac{R_{q}}{R}
$$

The Tow-Thomas structure allows the corner frequency and the $\mathbf{Q}$ of the filter to be adjusted independently. The filter used for the FSK demodulator board was designed to have $\mathrm{Q}$ and $\mathrm{DC}$ gain of one, with a comer frequency of $4 \mathrm{MHz}$. 


\subsubsection{Phase-shift Frequency T̃iscriminator}

Figure (5-12) shows a simplified circuit diagram of the ECL based phase-shift frequency discriminator.

Figure 5-12: Phase-shift Frequency Discriminator

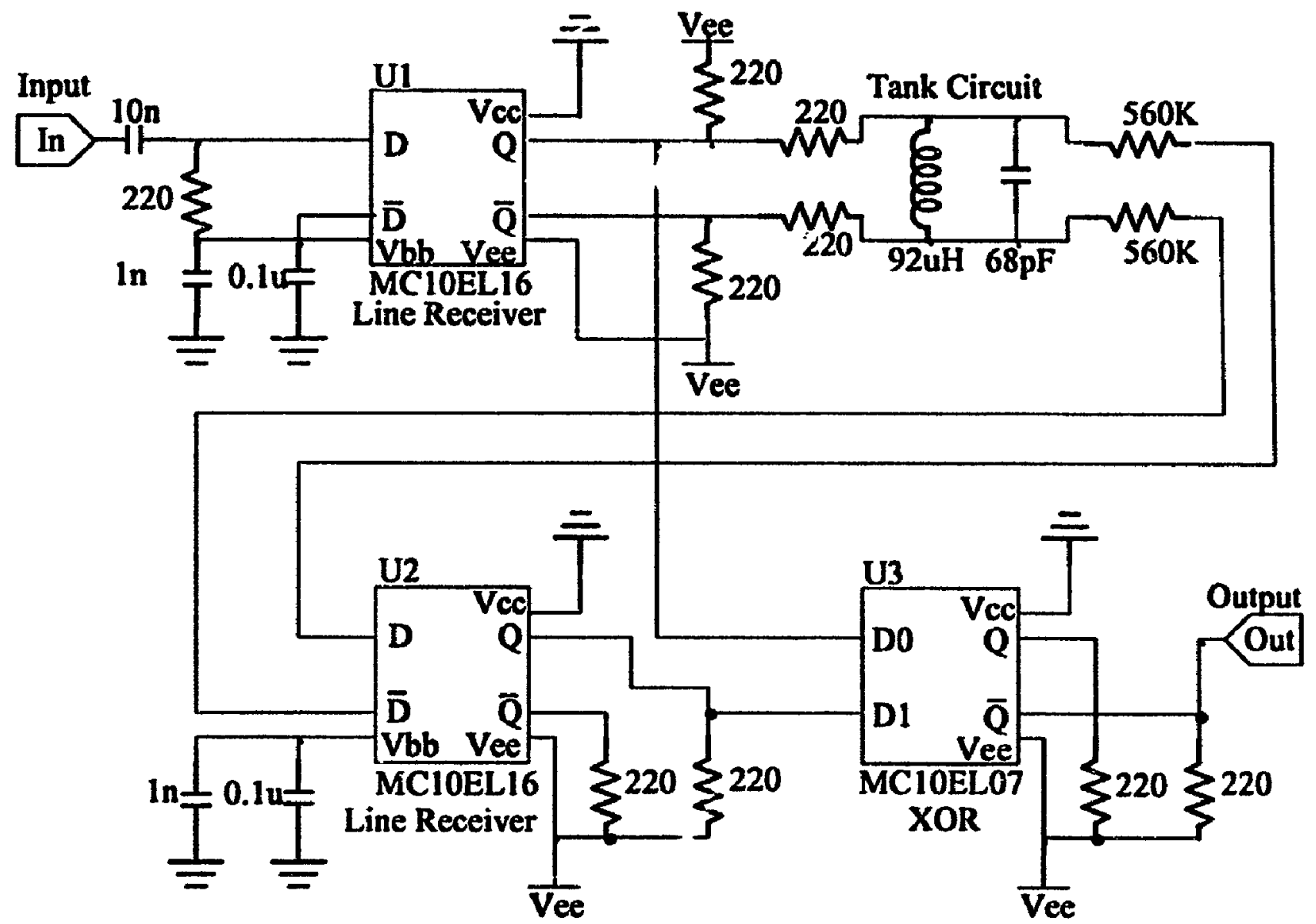

The first ECL line receiver is used to limit the amplitude of the received signal, eliminating residual AM. The output of the first line receiver is split into two separate paths. The first path consists of a fully differential LC tank circuit that provides a linear phase shift which is equal to zero degrees at the IF carrier frequency. The differential circuit structure is used in order to reduce the effects of common-mode noise in the circuit. The signal from the LC tank is then passed through another ECL line receiver in order to restore it to maximum amplitude. The output of the line receiver is then phase compared to the second non-phase 
shifted path using the ECL XOR gate shown. The family of ECL logic used did not have a fully differential two input XOR gate available, making the use of the single-ended version necessary. The resistors shown at the input and output to the LC circuit in Figure (5-12) are used to set the $Q$ of the tank circuit. It is useful to derive an approximate expression for the discriminator characteristic of the FSK demodulator board. This can be done by finding an expression that describes the phase shift of the LC tank circuit for a change in input frequency anc has units of radians $/ \mathrm{Hz}$. This can be combined with the theoretical phase cieiector chaaracteristic for the XOR gate, which has units of Volts/radians, in order to obtain ine overall discriminator characteristic which has units of Volts/Hz. The tank circuit was simulated using SPICE, and the amplitude and phase response plots obtained are shown in Figures (5-13) and (5-14) respectively.

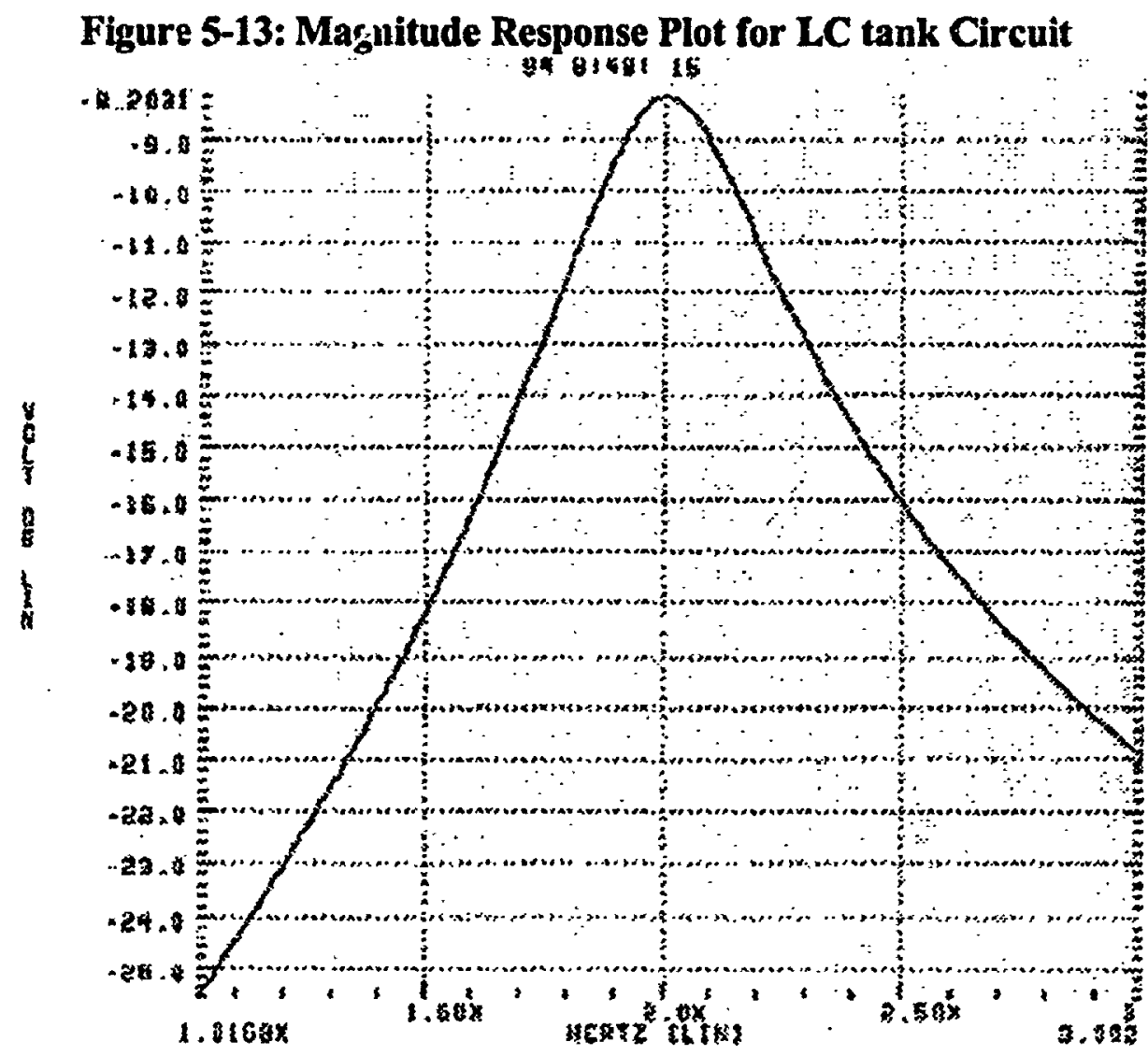




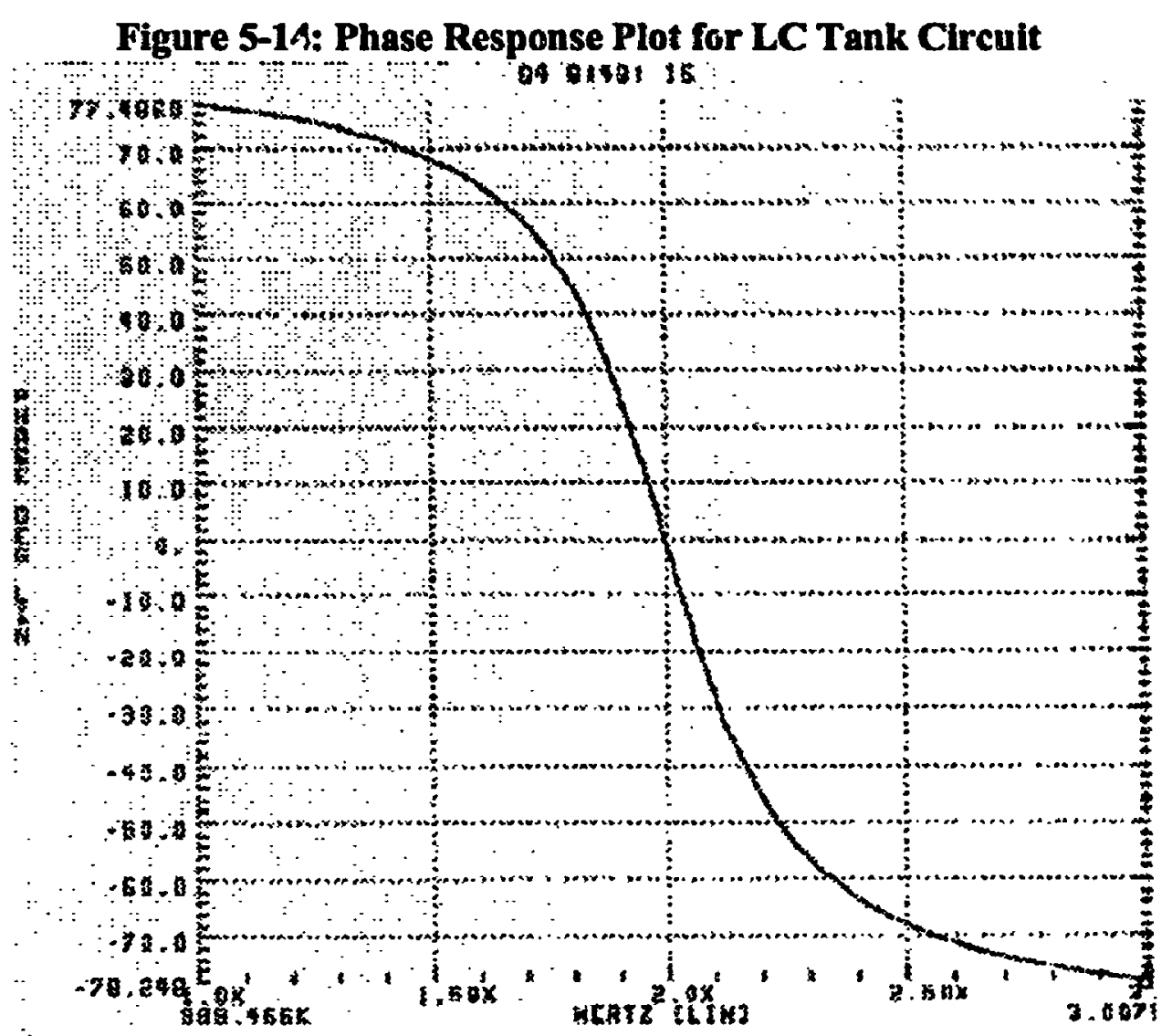

The phase shift per unit frequency is given by the slope of the phase response plot. Estimating the slope of the phase plot by a straight line extending from $+45^{\circ}$ to $-45^{\circ}$ over the $3 \mathrm{~dB}$ bandwidth of the filter, the phase characteristic, $\mathrm{K}_{\mathrm{p}}$, is approximately equal to;

$$
K_{p} \cong\left(\frac{\pi}{2}\right)\left(\frac{1}{2 \pi f_{b}}\right)=\frac{1}{4 f_{b}}(\text { radians } / \mathrm{Hz})
$$

Where $\mathrm{f}_{\mathrm{b}}$ is the 3-dB bandwidth of the filter in Hertz. The phase detector characteristic for an XOR gate, $K_{X}$, is given by [Bla92]; 


$$
K_{x}=2 \frac{A}{\pi} \text { (Volts/radians) }
$$

Where $A$ is the amplitude of the output signal from the decision circuit. If we multiply $K_{p}$ by $K_{x}$ we obtain the frequency discriminator characteristic, $K_{p d}$, which is equal to;

$$
K_{p d} \cong \frac{1}{4 f_{b}} \frac{(2 A)}{\pi}=\frac{A}{2 \pi f_{b}} \text { (Volts/radians) }
$$

Using the above equations, and the simulation results for the tank circuit, the approximate discriminator characteristic for the circuit designed can be calculated. Using the phase plot in Figure (5-14) we can calculate the slope of the phase characteristic for the simulated tank circuit using Equation (5-10). The slope is;

$$
K_{p} \cong \frac{1}{4\left(500 \times 10^{3}\right)}=5 \times 10^{-7}(\text { radians } / \mathrm{Hz})
$$

The output of the XOR gate has an amplitude of $500 \mathrm{mv}$ peak, and therefore the phase detector characteristic can be calculated using Equation (5-11) and is approximately equal to;

$$
K_{x}=\left(2 \frac{\left(500 \times 10^{-3}\right)}{\pi}\right)=0.32(\text { Volts/radians })
$$

The overall discriminator characteristic for the demodulator is calculated using Equation (5-12), and is approximately equal to; 


$$
K_{p d}=5.0 \times 10^{-7}(0.32)=160 \mathrm{nV} / \mathrm{Hz}
$$

The discriminator characteristic of the circuit was measured in the laboratory and is equal to $200 \mathrm{nV} / \mathrm{Hz}$, measured at the output of the carrier suppression filter. This is very similar to the predicted value. Note that the carrier suppression filter is passive, and therefore has insertion loss which reduces the amplitude of the output of the decision circuit.

\subsubsection{Passive Carrier Suppression Filter}

Figure (5-15) shows the circuit diagram for the passive lowpass filter that follows the output of the frequency discriminator. The required filter component values were obtained using a filter design software package developed by RF Design magazine. The filter has a 4th order Butterworth lowpass response with a corner frequency of $500 \mathrm{KHz}$. The normalized filter transfer function is;

$$
H(s)=\frac{1}{s^{4}+2.61 s^{3}+3.41 s^{2}+2.61 s+1}
$$


Figure 5-15: Passive Carrier Suppression Filter

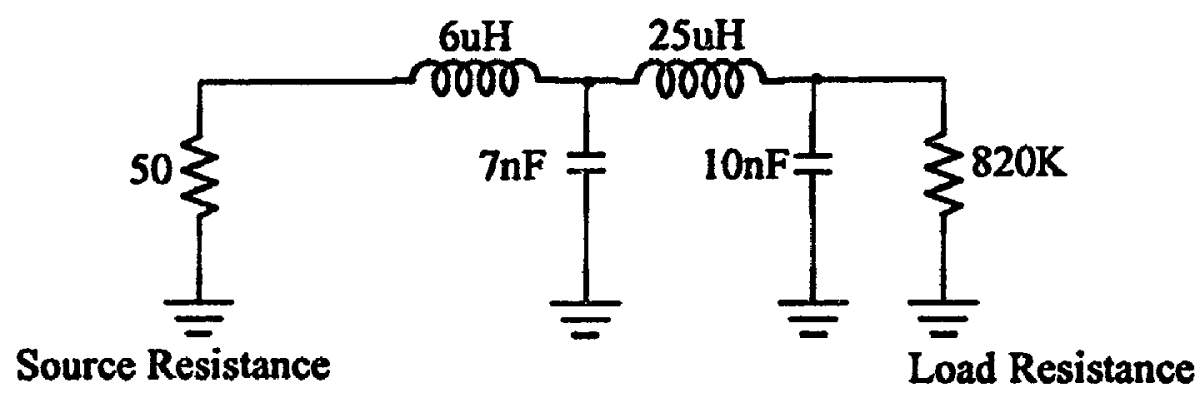

\subsubsection{Matched Filter and Decision Circuit}

The circuit diagram for the integrate and dump filter that follows the passive carrier suppression filter is shown in Figure (5-16). The filter integrates the input signal over the entire symbol period. The dump pulse generated by the BER board at the end of each symbol interval is used to turn on the transmission gate shown in Figure (5-16), dumping the charge stored on the integrating capacitor. The output of the comparator that follows the integrator output is latched when the rising edge of the dump pulse is detected. Figure (517) shows a stream of data bits from the discriminator and the resulting output from the integrate and dump filter, obtained from a SPICE simulation. Note that the component values used in the simulation are slightly different from those used in the circuit and therefore, no axis values are provided in Figure (5-17). Because the integrator responds to the average value of the input signal, it is very insensitive to white Gaussian noise which theoretically has an average value of zero. This form of symbol detection provides a high level of robustness in the demodulator when the system operates in a white noise environment. 
Figure 5-16: Matched Filter and Decision Circuit

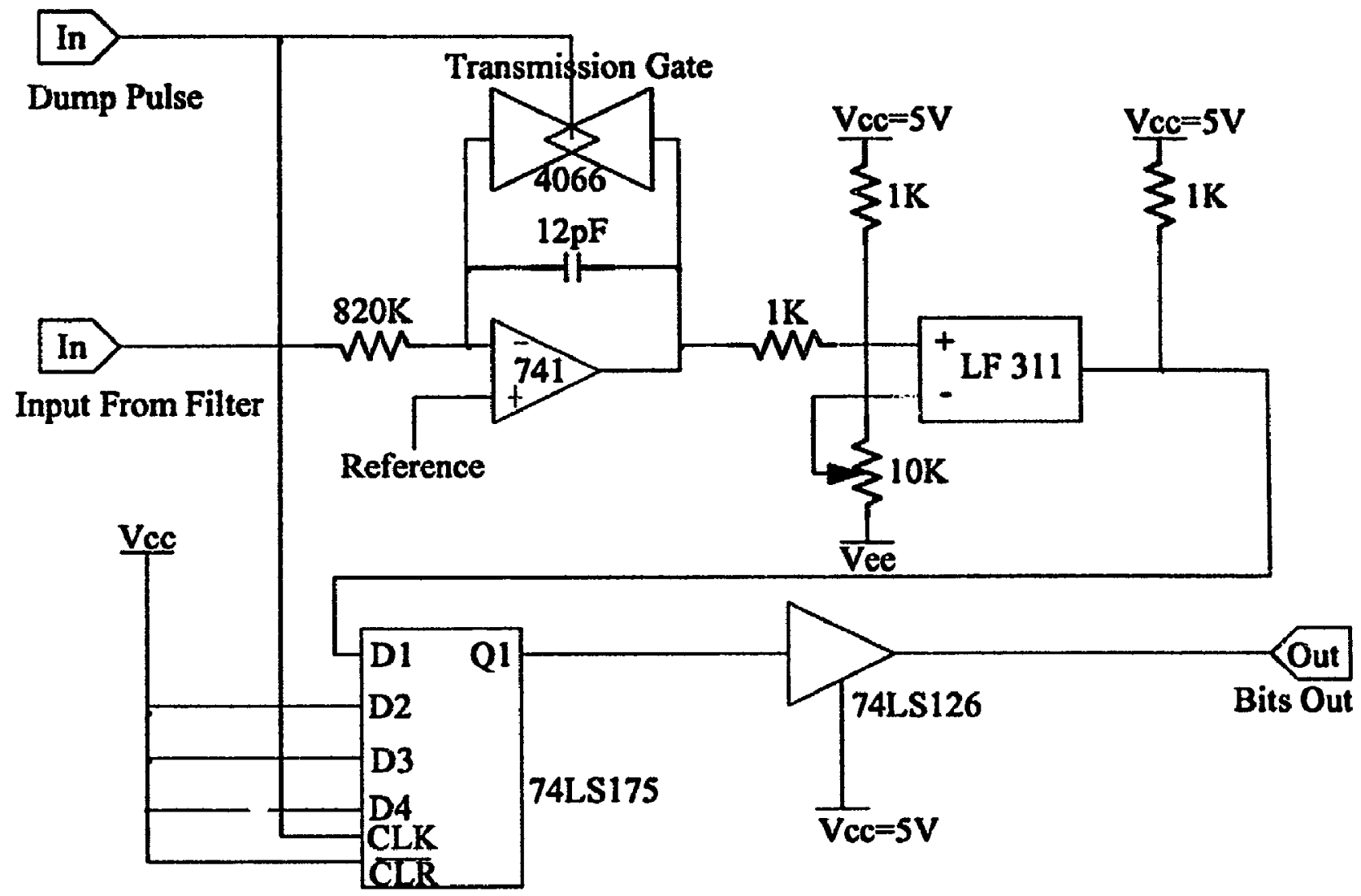

Figure 5-17: Output of Integrate and Dump Fiter

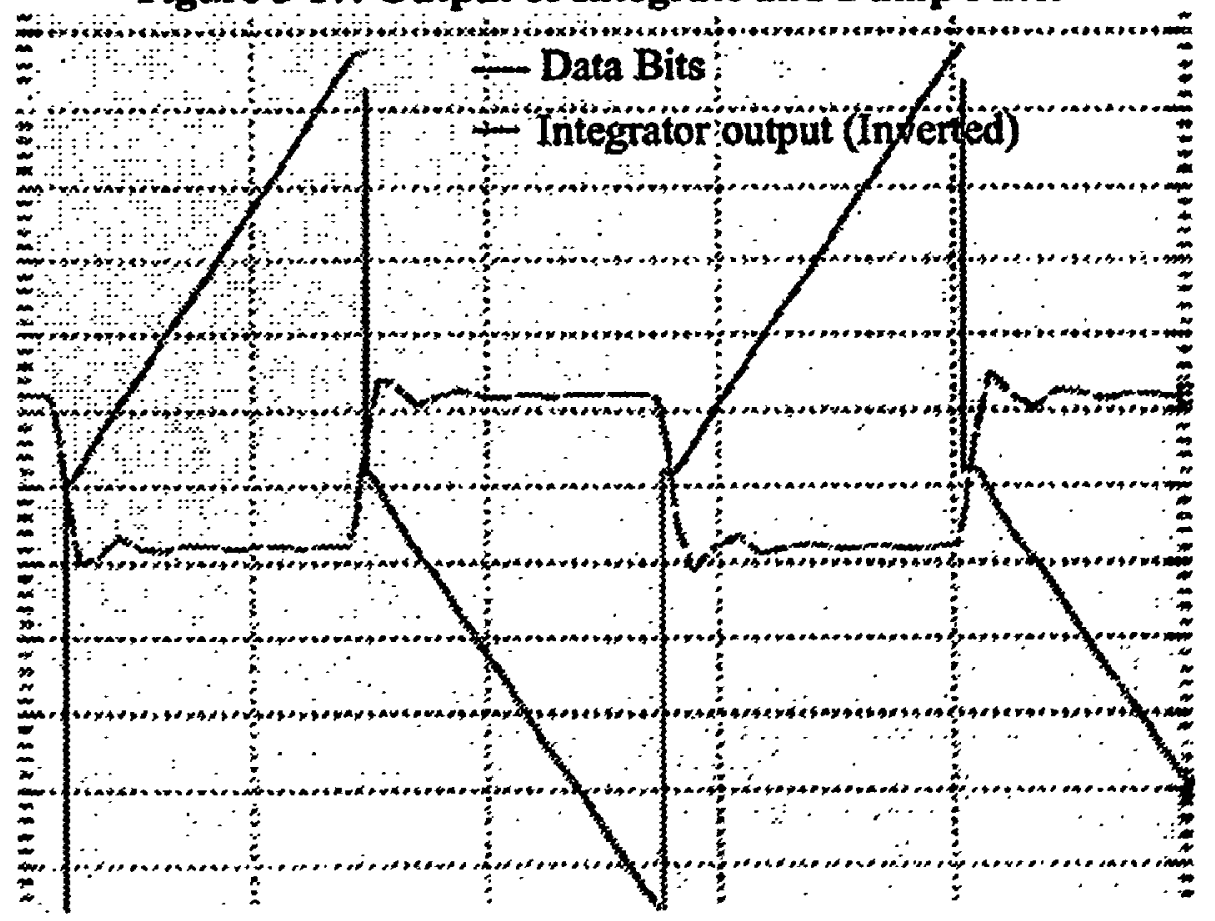




\section{Chapter 6 \\ Results}

\subsection{Simulation Results}

The following sections present the results from the simulations of simple frequencyhopping and interpolated frequency-hopping that were performed using MATLAB, a mathematical analysis program which runs on UNIX and DOS based computer systems.

\subsubsection{Simulation Methods}

MATLAB has a large number of analysis functions that are grouped together under a single software package called "The signal-processing toolbox". This toolbox has powerful functions that allow a user to specify system transfer functions, and to filter data using these functions. Operations can be performed on signals in both the frequency domain and the time domain, and graphical plots of the results can be obtained.

An approximate closed-loop transfer function for the PLL used in the test system shown in Figure (6-1) was derived. A second transfer function was then specified which is almost identical to the first transfer function, with a certain degree of mismatch introduced. The first transfer function represents the transmitter of the spread-spectrum system, and the second almost identical transfer function represents the local oscillator in the receiver of the spread-spectrum system. Two arrays are created. The first array consists of the desired hopping sequence for the carrier (frequency vs. time), along with data if desired. The second array stores the hopping sequence along with an IF offset. The first array is then filtered by the transmitter transfer function, and the second array is filtered by the local oscillator transfer function. The arrays that are obtained from the filtering operations are then subtracted from one another, simulating the despreading process that takes place in the receiver of the spread-spectrum system. The array which is obtained from the subtraction operation contains the IF frequency information, including transmitted data and frequency error due to the mismatch between the two transfer functions. Fourier transform techniques are then used to obtain the frequency spectrum of the despread IF. The demodulated 
baseband spectrum is also obtained, assuming a discriminator characteristic of $1 \mathrm{~V} / \mathrm{Hz}$. In order to obtain accurate frequency spectrums from the information in the IF array, multiple FFTs and ensemble averages were performed. It should be noted that the hopping rates, the hop sizes, and the data rates used in the simulations were all integer sub-multiples of the 10 MHz sampling frequency used in the system, and that they have been rounded to integer values for easier presentation in the thesis.

\title{
6.1.2 PLL Model
}

Figure (6-1) shows a block diagram/schematic which is an approximate model of the frequency synthesizer used in the test system.

\section{Figure 6-1: Synthesizer Model}

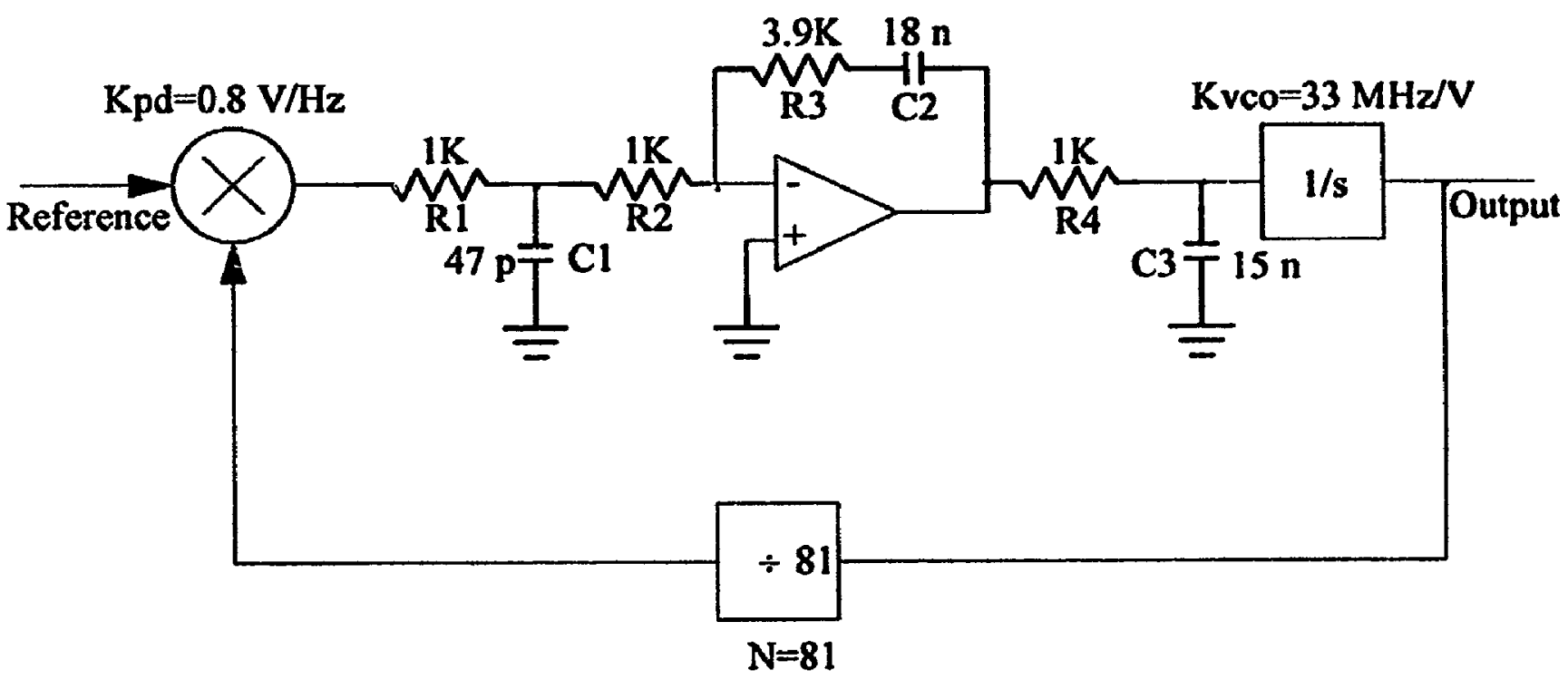

\begin{abstract}
Although the synthesizers used in the test system were controlled digitally using a multiple modulus divider, the input signal for the model shown in Figure (6-1) is assumed to be the reference frequency, with a fixed frequency divider present in the feedback path of the loop. This assumption simplifies the analysis of the loop. The closed-loop PLL transfer function, from the reference frequency input to the output of the VCO, is given by;
\end{abstract}




$$
H_{c l}(s)=\frac{K F(s)}{s+K F(s)}
$$

Where $\mathrm{K}$ is given by;

$$
K=\frac{K_{P D} K_{v C O}}{N}
$$

Where $\mathrm{K}_{\mathrm{PD}}$ is the phase detector constant, and $\mathrm{K}_{\mathrm{VCO}}$ is the VCO tuning c nnstant. $\mathrm{F}(\mathrm{s})$ is the loop filter transfer function given by;

$$
F(s)=-\frac{1}{s \tau_{2}}\left[\frac{1+s \tau_{3}}{1+s \tau_{1}}\right]\left[\frac{1}{1+s \tau_{4}}\right]
$$

Where $\tau_{1}, \tau_{2}, \tau_{3}$, and $\tau_{4}$ are the filter time constants given by;

$$
\tau_{1}=\frac{\left(R_{1} R_{2} C_{1}\right)}{R_{1}+R_{2}}
$$

$$
\tau_{2}=C_{2}\left(R_{1}+R_{2}\right)
$$




$$
\tau_{3}=C_{2} R_{3}
$$

$$
\tau_{4}=C_{3} R_{4}
$$

The PLL used in the test system is actually a Sth order loop, but for the model used in the simulations, two of the poles were ignored as they are not dominant and have very little effect within the loop bandwidth.

\subsubsection{Simulation of the Carrier}

A MATLAB simulation of simple frequency-hopping and the equivalent interpolated frequency-hopping transmission was performed for ten frequency steps in the frequency range of the experiment, assuming a synthesizer loop filter component mismatch of $10 \%$ and a noiseless system. Here, "simple" means no guard times, ping-ponging, etc. The hopping rate used in the simulation was $10 \mathrm{KHz}$ with maximum hop sizes ranging from 10 $\mathrm{KHz}$ to $600 \mathrm{KHz}$. The simulated frequency versus time plots for the transmitted signals are shown in Figure (6-2) for the two alternatives. 
Figure 6-2: Simple and Interpolated Frequency-hopping (Frequency vs. Time)

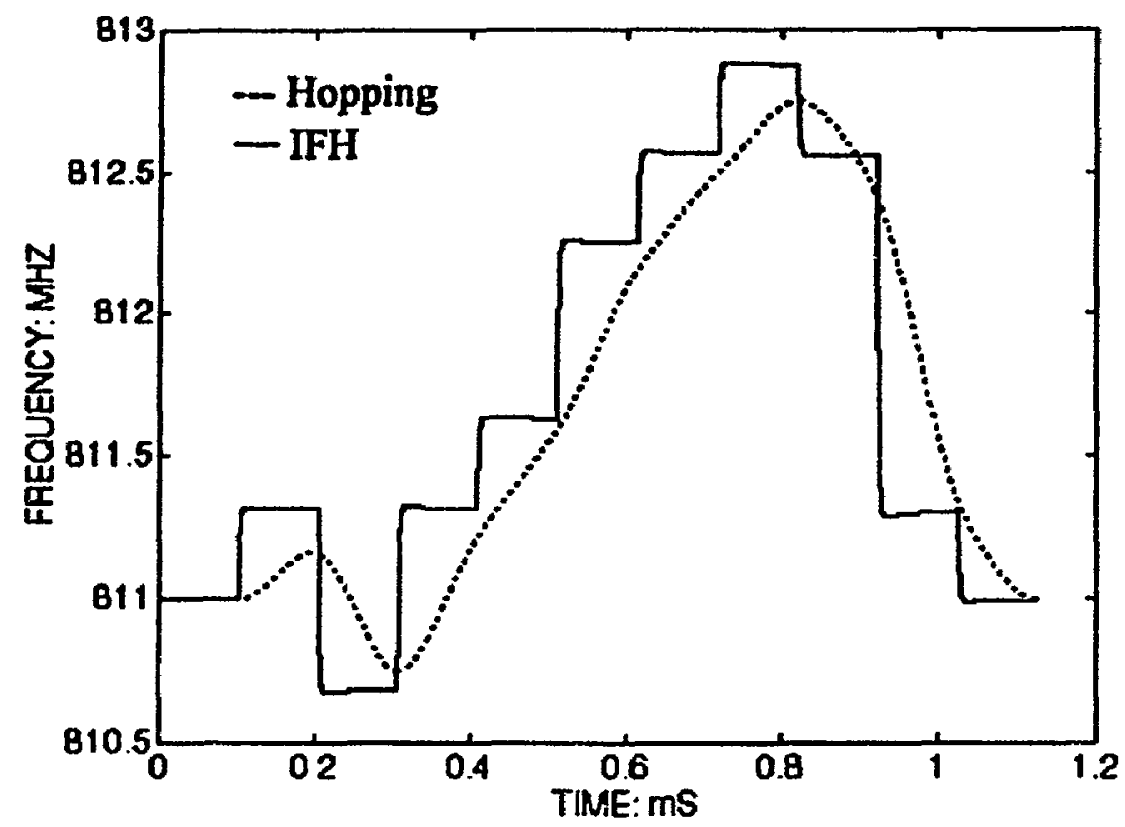

The incretsed smoothness of the carrier movement for interpolated frequency-hopping transmission is clearly seen on the plot. Figure (6-3) shows a plot of the instantaneous phase versus time for both simple frequency-hopping and interpolated frequency-hopping. 
Figure 6-3: Simple and Interpolated Frequency-hopping (Phase vs. Frequency)

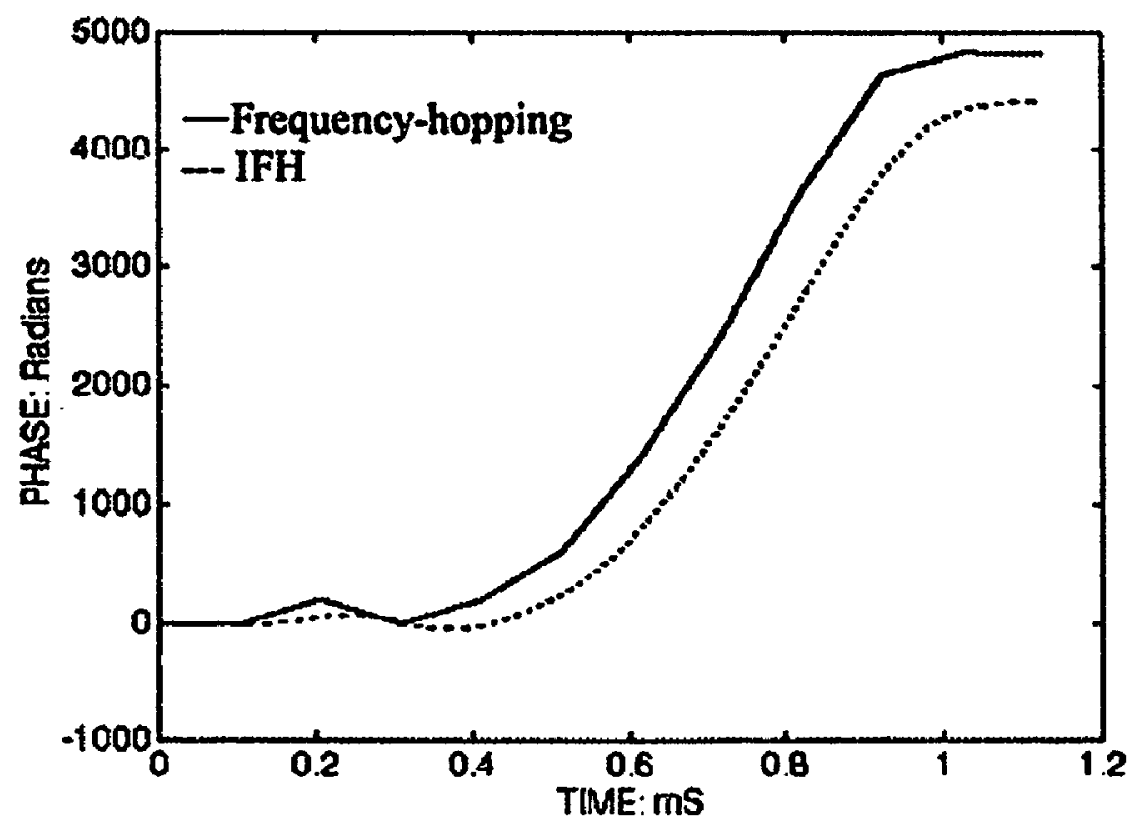

It can be seen from Figure (6-2) and Figure (6-3) that interpolated frequency-hopping is both a continuous frequency and a continuous phase form of carrier modulation. Figure (64) shows a plot of the simulated instantaneous IF frequency error for simple frequencyhopping and interpolated frequency-hopping, assuming ideal mixing, for the frequency steps shown in Figure (6-2). As predicted, the IF frequency-error present in simple frequency-hopping transmission takes the forn oi sharp bursts occurring at the $10 \mathrm{KHz}$ code rate used in the simulation. For interpolated frequency-hopping, these error bursts are replaced by low level, almost constant frequency error, as Figure (6-4) demonstrates.

Figures (6-5), (6-6), and (6-7) show the simulated IF spectrums for both simple frequencyhopping and interpolated frequency-hopping for a $10 \mathrm{KHz}$ code rate, and maximum hop sizes of $1.25 \mathrm{MHz}, 2.5 \mathrm{MHz}$, and $5 \mathrm{MHz}$ respectively. For maximum hop sizes of $5 \mathrm{MHz}$, the underlying triangular frequency distribution is not used. Quantization noise, and a $10 \%$ component mismatch between the two loop filters is considered. 
Figure 6-4: Simple and Interpolated Frequency-hopping

(IF Frequency-error vs. Time)

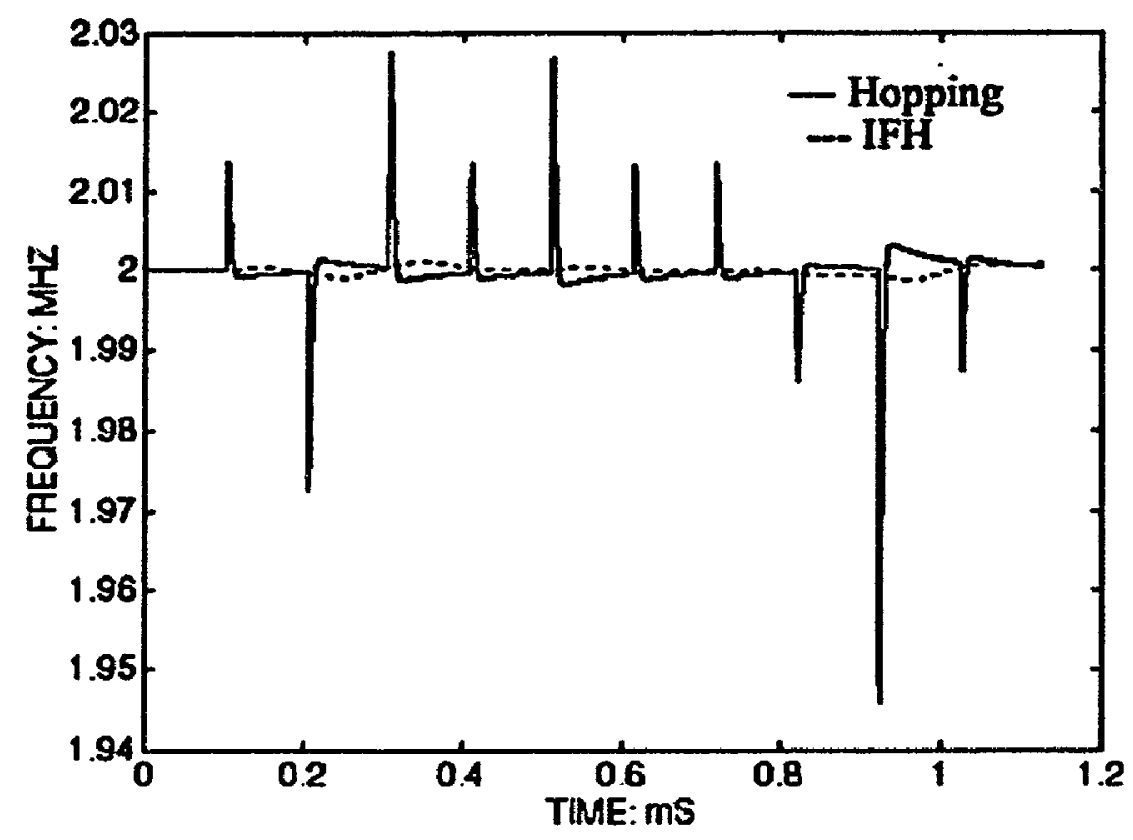

Figure 6-5: IF Spectrum Without Data

(Hop rate=10 KHz, Maximum Hop size $=1.25 \mathrm{MHz}$ )

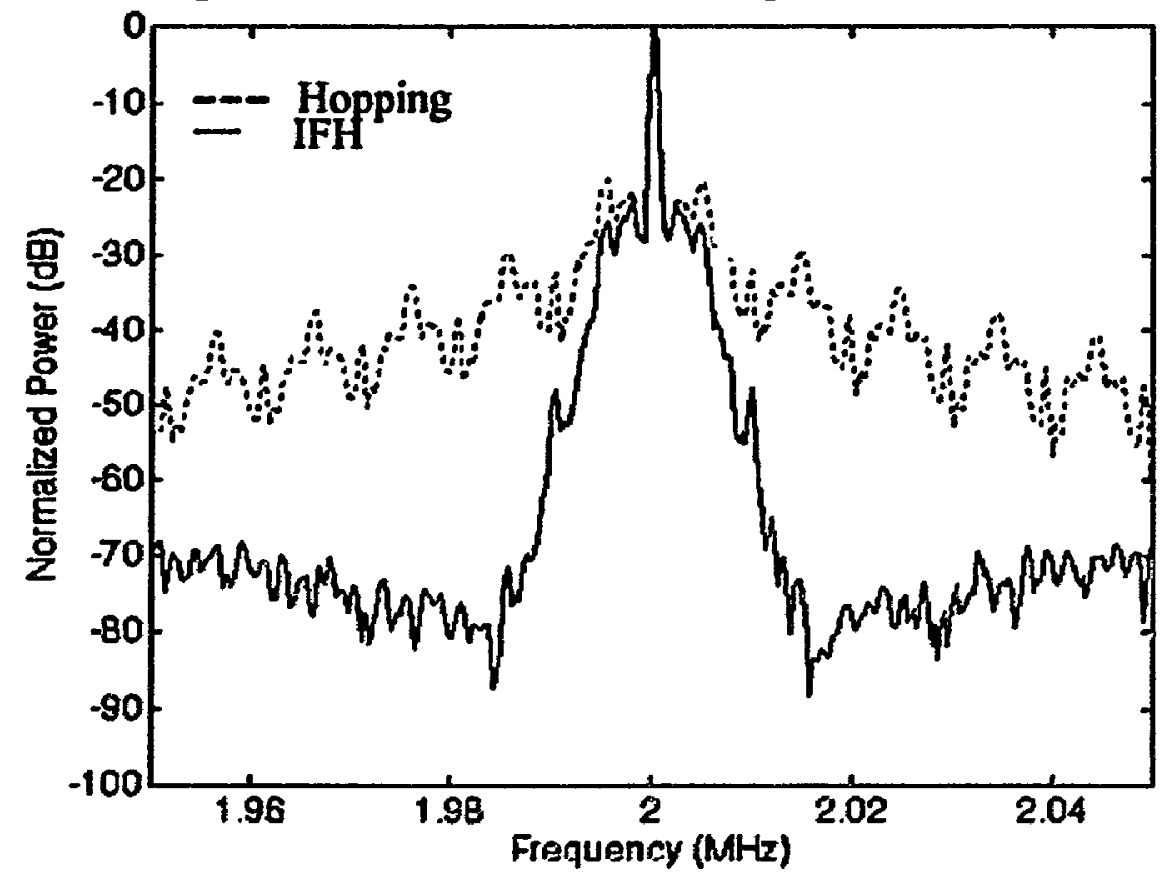


Figure 6-6: IF Spectrum Without Data

(Hop rate=10 KHz, Maximum Hop size=2.5 MHz)

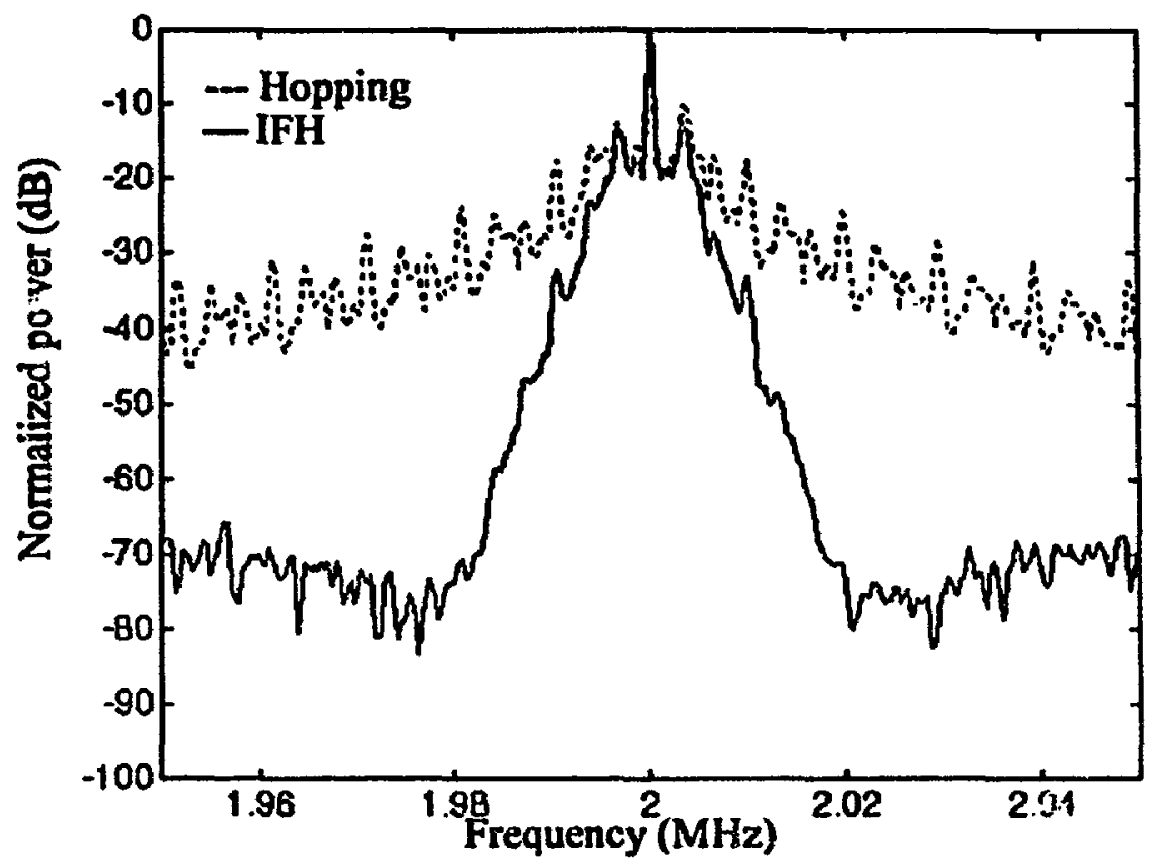

Figure 6-7: IF Spectrum '.ithoua Data

(Hop rate=10 KHz, Maximum Hop size=5.0 MHz)

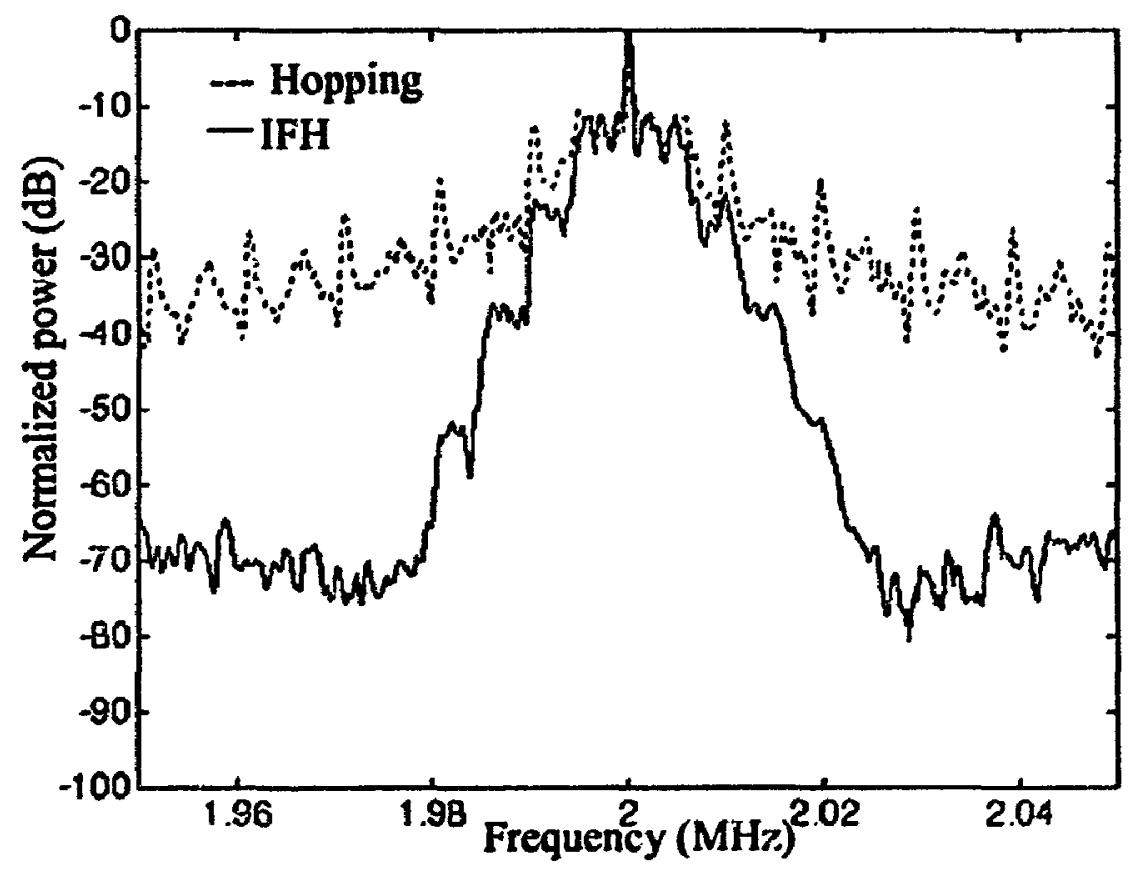



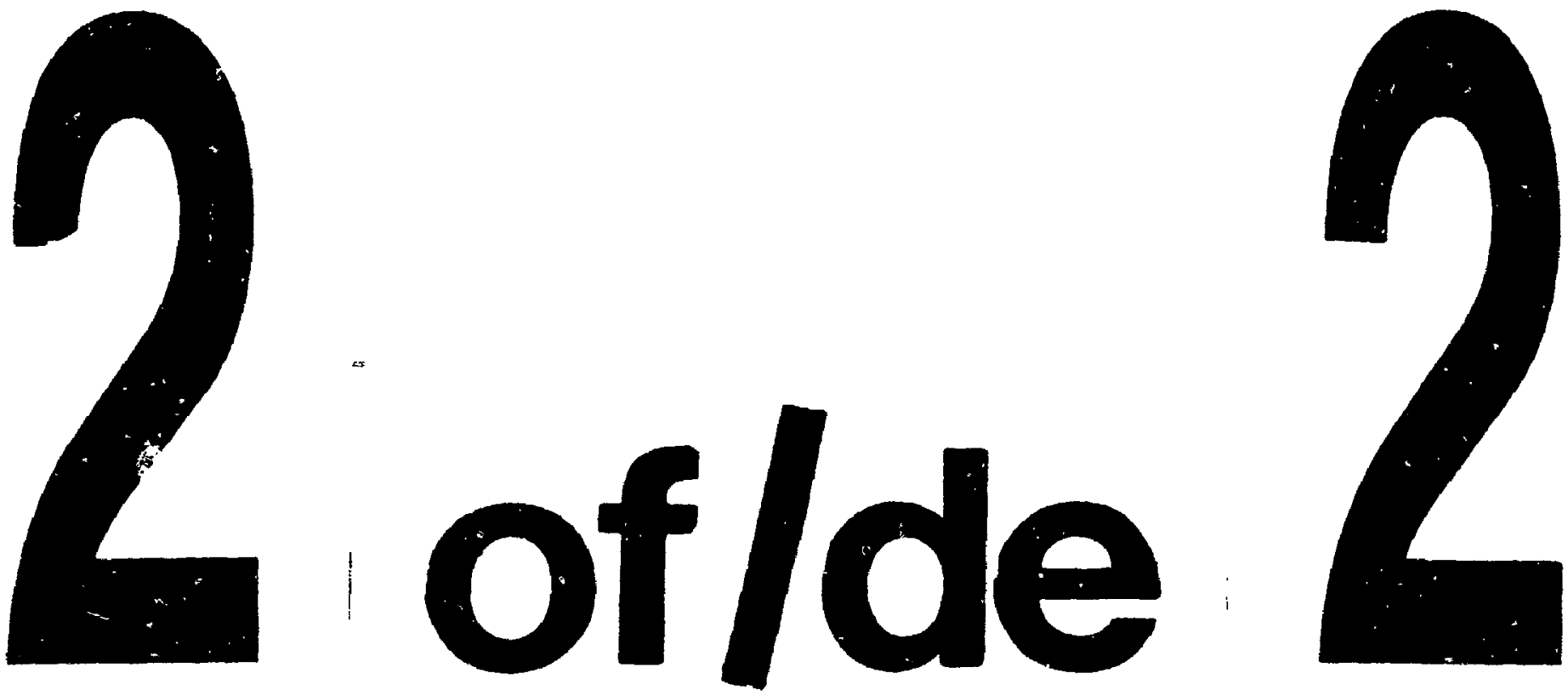

PM-1 31/2"X4" PHOTOGRAPHIC MICAOCOPY TARGET NBS 1010a ANSI/ISO \#2 EOUIVALENT

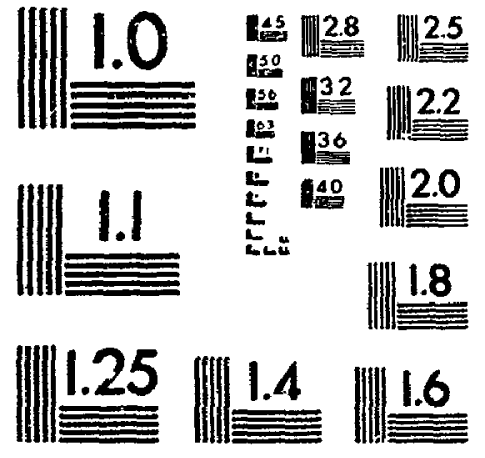

PRECISIONSM RESOLUTION TARGETS 
The simulations clearly slow that interpolatios: of the hopping code reduces the IF distortion caused by synthesizer mismatch. Figure (6-8) shows the simulated IF spectrum for both simple frequency-hopping and interpolated frequency-hopping when the natural frequency of the loop filter in one of the synthesizers is reduced by a factor of $\sqrt{2}$, compared to the other. The maximum hop size for this case is $1.25 \mathrm{MHz}$.

Figure 6-8: Natural Frequency Mismatch

(Rate=10 KHz, Maximum Hop size $=1.25 \mathrm{MHz}$ )

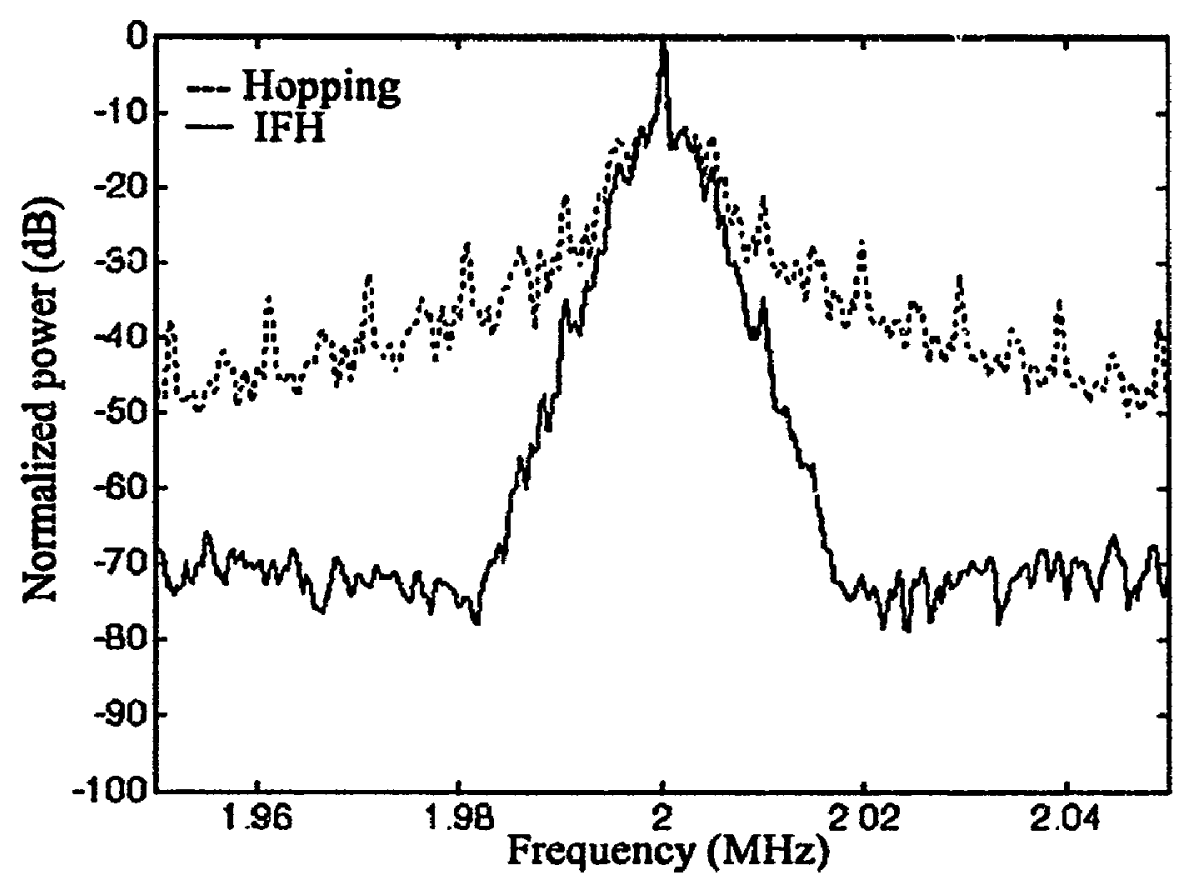

It can be seen from Figure (6-8) that the quality of the IF spectrums for both simple and interpolated frequency-hopping are degraded when compared to the ones shown in Figure (6-5), particularly for close-in phase noise (i.e., within $5 \mathrm{KHz}$ of the IF). This shows that in order for interpolated frequency-hopping to work well, the synthesizers used should have identical loop filter designs, and be carefully matched. This constraint is more important for interpolated frequency-hopping than for simple frequen:jy hopping, because in interpolated frequency-hopping the data is continuously sampled, whereas in simple frequency-hopping the data is only sampled when the transient phase-error has settled out to steady-state. 
If the transmitter and receiver synthesizers were identical, there would be no distortion present in the receiver IF. However, in a real system, identical transient response between two synthesizers is impossible to achieve. The result of the mismatch between the two synthesizers is an unwanted, attenuated version of the hopping code present in the receiver IF. If the code length was infinite, the hopping would be non-deterministic in nature and the IF spectrum would have a continuous $\sin x / x$ form.

As a reality check, a simulation was performed for both simple frequency-hopping and interpolated frequency-hopping using identical loop transfer functions for both the transmitter and recsiver, and no quantization noise. The resulting frequency spectrum consisted only of the IF centre frequency with no degradation present due to incomplete despreading.

\subsubsection{Simulation of Carrier With Data}

Simulations were performed for both simple frequency-hopping and interpolated frequency-hopping with a hopping rate of $10 \mathrm{KHz}$, a maximum hop size of $1.25 \mathrm{MHz}$, and random binary-FSK data added to the carrier. The data rate used was $78 \mathrm{~Kb} / \mathrm{s}$ with frequency deviations of $+1-500 \mathrm{KHz}$. The transmitted data was not filtered. Figure (6-9) shows the simulated IF spectrum for the carrier and the data. Figure (6-10) shows the simulated baseband spectrum of the demodulated data, assuming an ideal discriminator characteristic of $1 \mathrm{~V} / \mathrm{Hz}$. 
Figure 6-9: IF Spectrum for Carrier and Binary-FSK Data

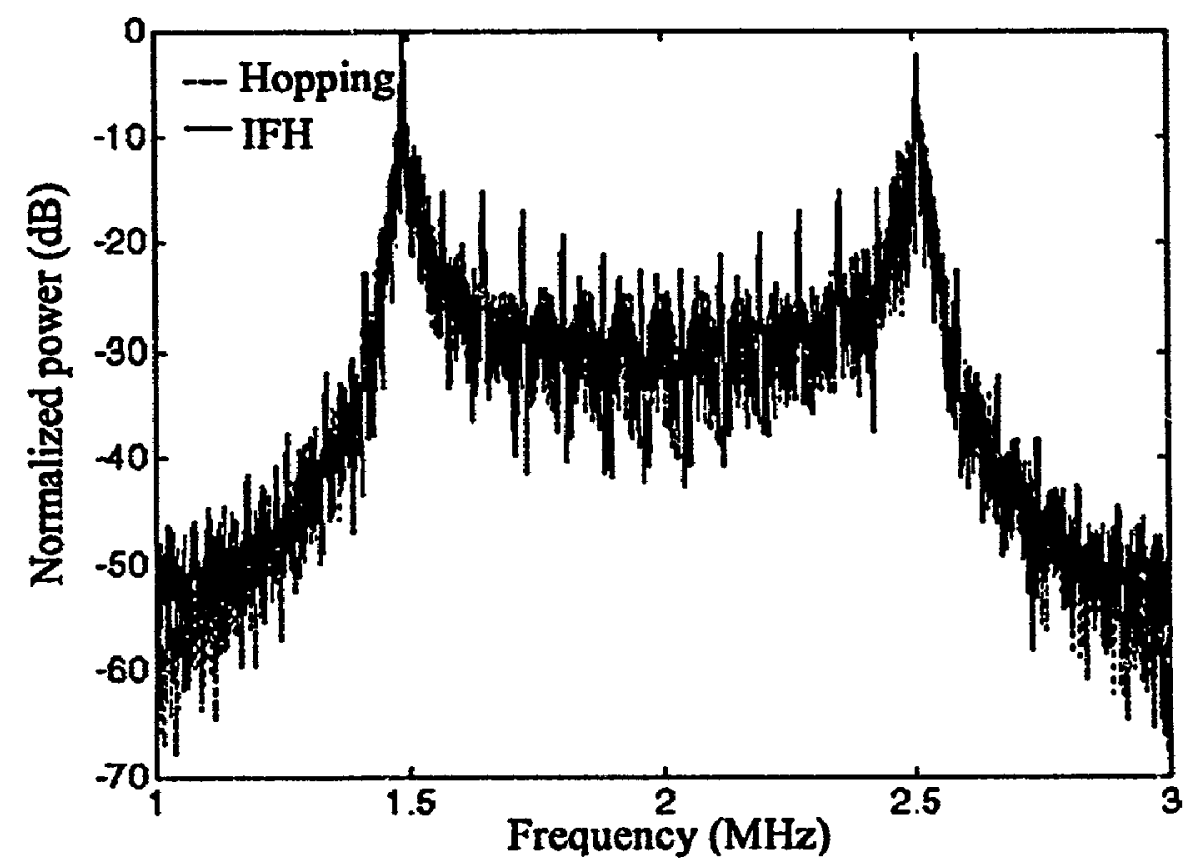

Figure 6-10: Baseband Spectrum for Demodulated Binary-FSK Data

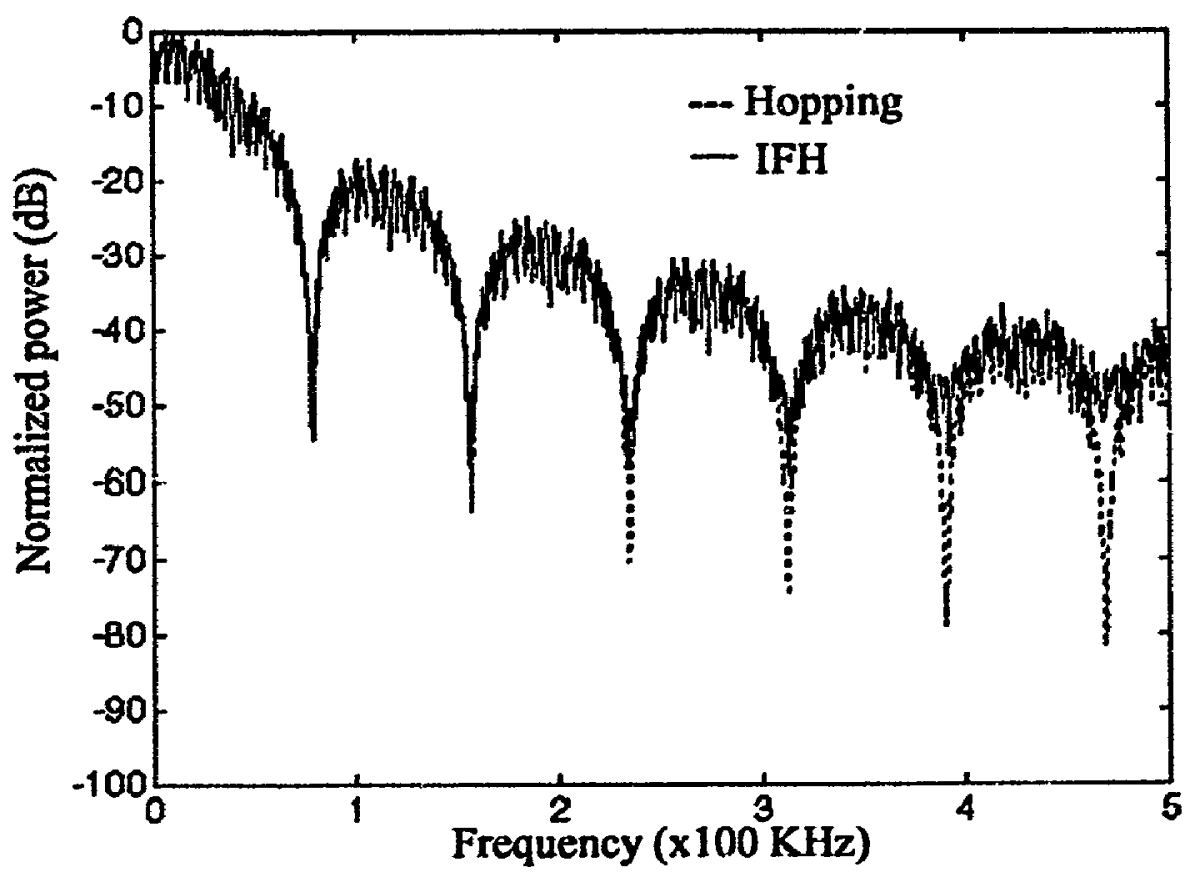

Because of the large data frequency deviations used in the sinulation, the IF distortion caused by synthesizer mismatch has very little noticeshle effect on the IF and baseband spectrums shown in Figures (6-9) and (6-10), respectively. Therefore, only the plots for the 
1.25 MHz hop size are presented.

\subsection{Laboratory Measurements}

Laboratory measurements of the relative transmission quality obtained through the use of simple frequency-hopping and interpolated frequency-hopping transmission were obtained using the setup described in the design scetion and shown in Figure (5-1). Similar measurements were performed using a stationary carrier, in order to establish a common reference point.

An array of frequency points uniformly distributed between upper and lower frequency limits selected in software was created, and added to a triangular waveform with a repetition time of $102 \mathrm{~ms}$. Individual hop sizes varied from $10 \mathrm{KHz}$ to $5.0 \mathrm{MHz}$, and the code rates used were $10 \mathrm{KHz}, 78 \mathrm{KHz}$, and $156 \mathrm{KHz}$. As in the simulations, the hopping rates, the hop sizes, and the data rates used in the laboratory test system are all integer submultiples of the $10 \mathrm{MHz}$ sampling frequency used in the system, and have been rounded to integer values for easier presentation in the thesis. Different values for the hop sizes and codes rates were used in order to determine the limits of the transmission methods and system used. Although the triangular waveform used is a non-standard frequency-hopping code, it provided a uniform frequency distribution and substantial simplification to the interpolation filter, as explained in the design section. As the hop sizes grow larger, because the total spreading bandwidth available to the system is fixed, the size of the underlying triangular waveform becomes smaller and for hop sizes of $5 \mathrm{MHz}$ is no longer present. The interpolation filter used for code smoothing was a Gaussian lowpass filter with a corner frequency equal to one-third of the hopping rate, and double-precision valued tap coefficients.

Two stored sequences of $2^{20}$ bits obtained from the software $\Delta-\Sigma$ modulator were used in real time to control the dual modulus dividers of the transmit and receive synthesizer boards. 


\subsubsection{Measurements Without Data}

In order to determine the spectral quality of the frequency synthesizers used in the test system, single sideband phase-noise measurements were performed for both the transmit and receive synthesizers, using the HP3048A phase-noise measurement system. Figures (611) and (6-12) present plots of the phase-noise measured for the transmit synthesizer in integer-N operation at $800 \mathrm{MHz}$, and fractional-N operation at $811 \mathrm{MHz}$, respectively. The phase-noise measurements performed for the receive synthesizer yielded almost identical results and therefore are not presented here.

Figure 6-11: Phase Noise Measurement for Tx Synthesizer in Integer-N Operation

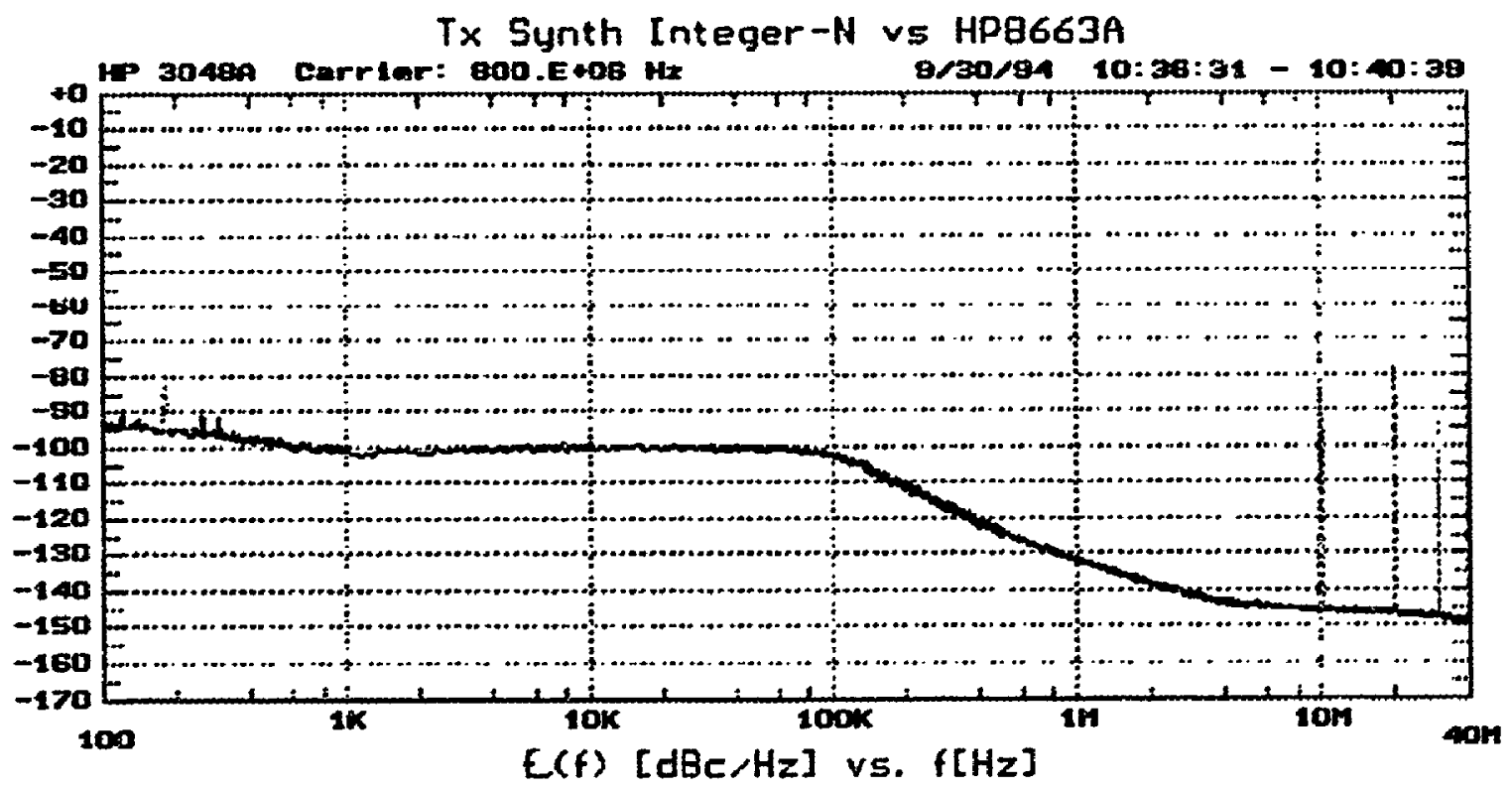


Figure 6-12: Phase Noise Measurement for Tx Synthesizer in Fractional-N Operation

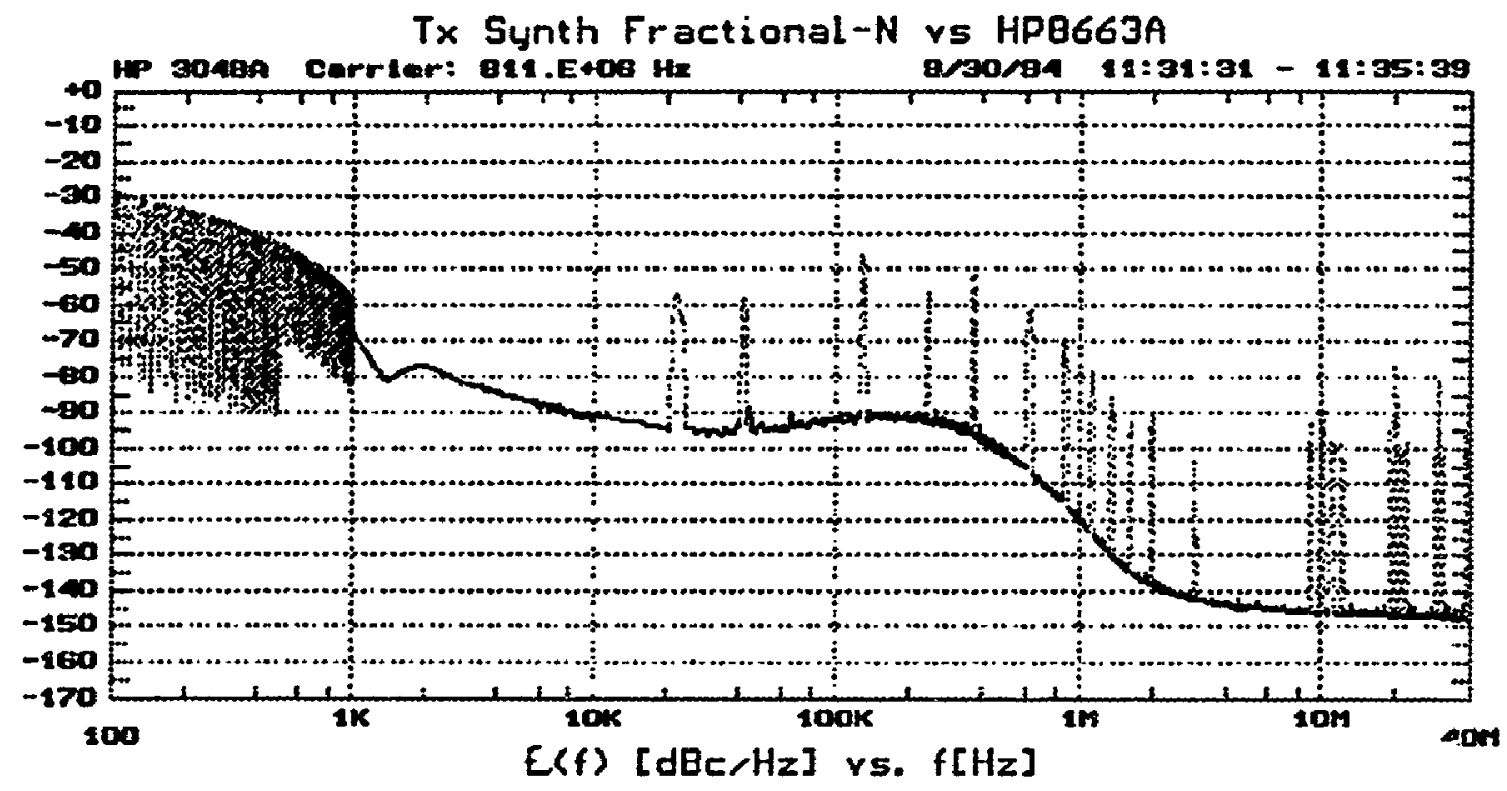

It is important to note that in fractional-N operation, the phase-noise plot for the transmit synthesizer shown in Figure (6-12) has a high level of phase-noise present within about 1 $\mathrm{KHz}$ of the centre frequency. This phase-noise is actually a series of spurs occurring at 10 $\mathrm{Hz}$ spacings, and is caused by the finite length of the synthesizer control bitstream stored in the bit generator board shown in Figure (5-1). In an actual implementation of this radio system, the repetition "glitch" would not be present in the control bitstream, and the phasenoise performance of the synthesizer in fractional- $N$ operation would improve significantly.

A plot of the measured frequency versus time characteristic for interpolated frequencyhopping transmission using the triangular coding waveform, with a hopping rate of $10 \mathrm{KHz}$ and a maximum hop size of $600 \mathrm{KHz}$, was obtained using a time interval analyzer and is shown in Figure (6-13). 
Figure 6-13: Hopping Code (Frequency vs. time)

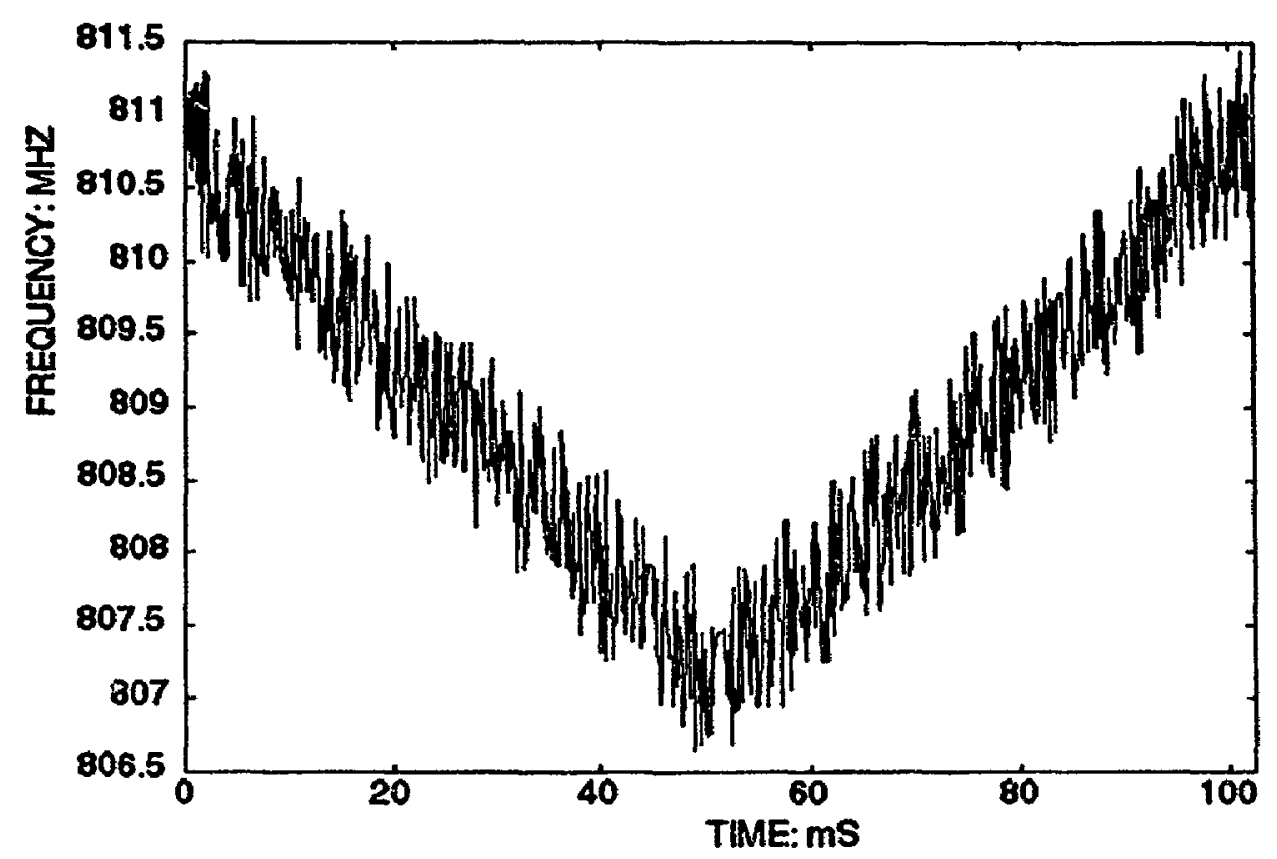

The time interval analyzer does not have the measurement resolution required to view individual frequency hops. Because of this, the plot for interpolated frequency-hopping transmission appears to be identic 1 to the one obtained for simple frequency-hopping, which therefore is not shown here. Plots obtained for higher frequency deviations are not shown because they do not vary significantly from the plot shown in Figur: (6-13) except for a widening of the frequency deviation.

A plot of the measured IF frequency spectrum for transmission using a stationary carrier is shown in Figure (6-14), and is included here for reference purposes in order to give an indication of the spectral purity of the IF obtained when frequency-hopping transmission is not employed. 
Figure 6-14: IF Spectrum for Stationary Carrier

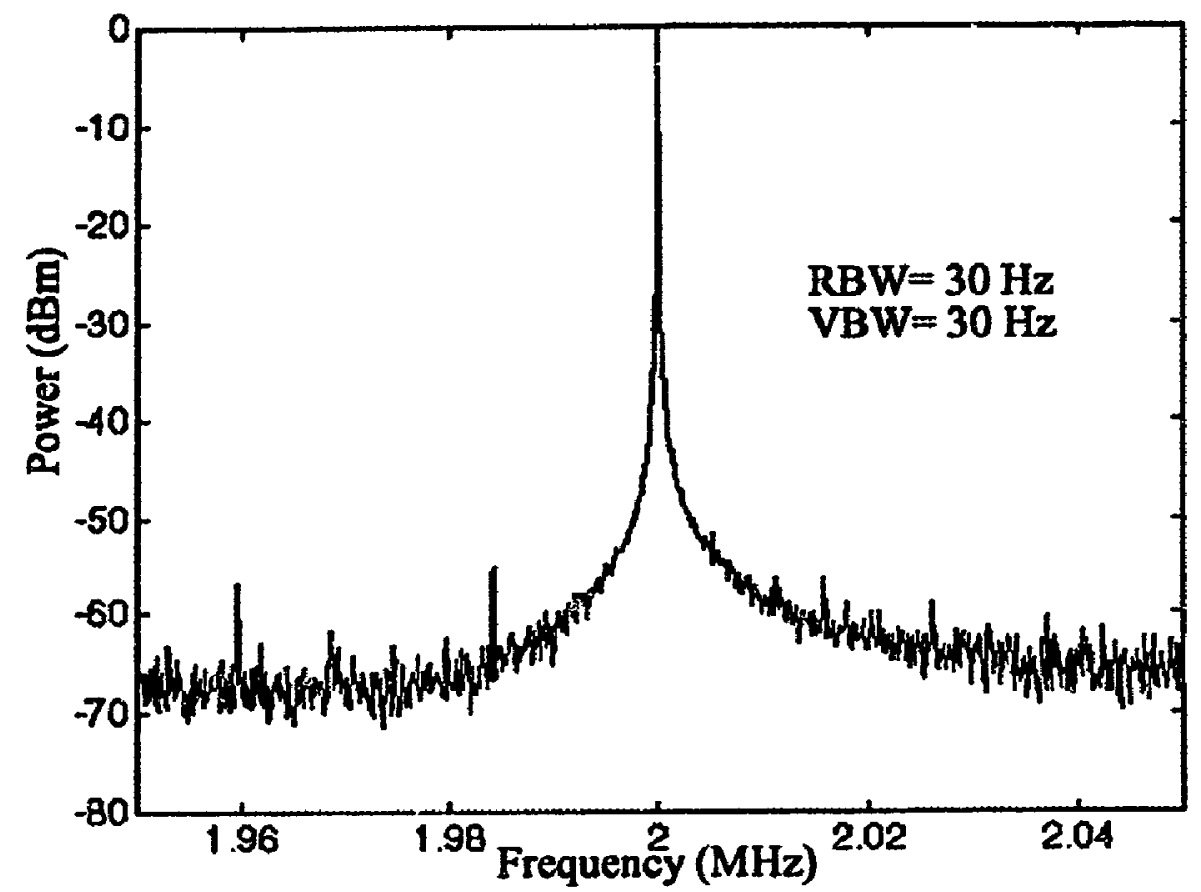

The absolute power level at the IF centre frequency is $-0.6 \mathrm{dBm}$, and the phase-noise is approximately equal to $-75 \mathrm{~dB} / \mathrm{Hz} @ 10 \mathrm{KHz}$.

Plots of the measured IF frequency spectrum including both simple frequency-hopping and interpolated frequency-hopping are shown in Figures (6-15) to (6-25). Plots are given for maximum hop sizes of $1.25 \mathrm{MHz}, 1.75 \mathrm{MHz}, 2.5 \mathrm{MHz}$ and $5.0 \mathrm{MHz}$ for each of the hopping rates of $10 \mathrm{KHz}, 78 \mathrm{KHz}$, and $156 \mathrm{KHz}$. Note that for the $10 \mathrm{KHz}$ hopping rate no measurement was made for a $1.75 \mathrm{MHz}$ hop size. 
Figure 6-15: IF Spectrum (Hopping rate $=10 \mathrm{KHz}$, Maximum Hop size=1.25 MHz)

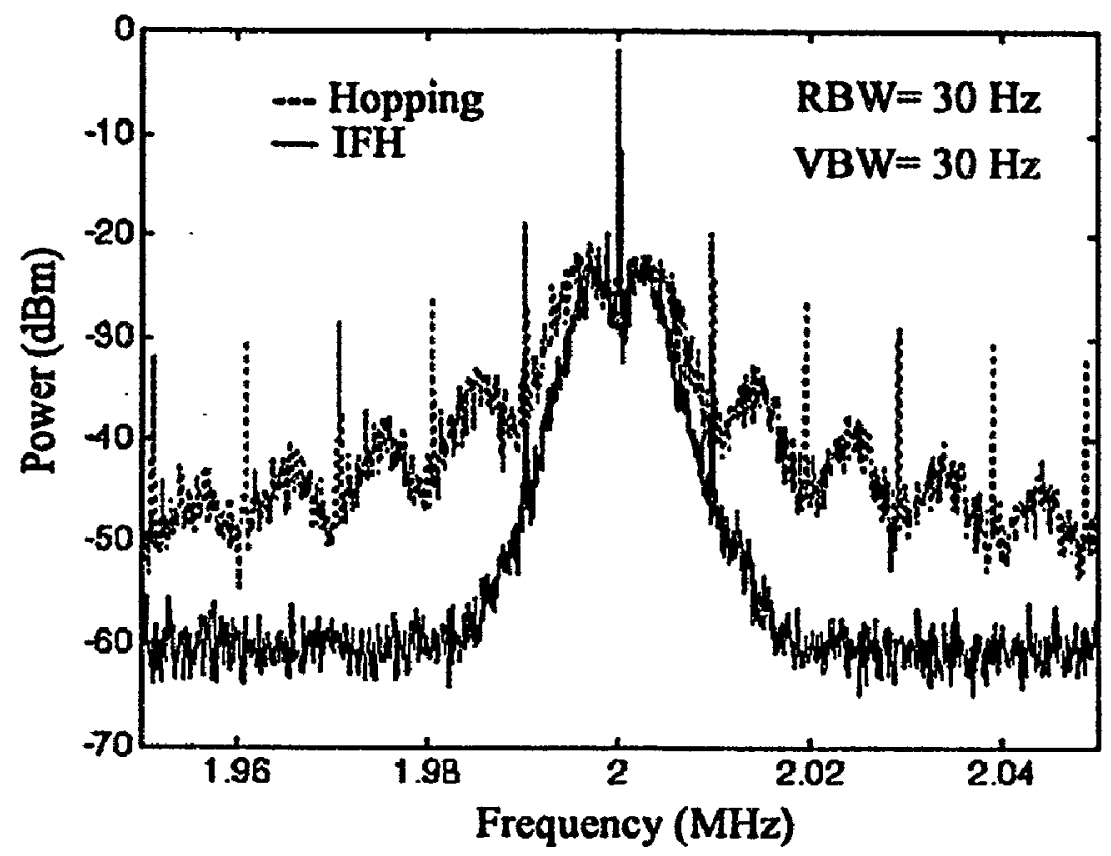

Figure 6-16: IF Spectrum (Hopping rate=10 KHz, Maximum Hop size=2.5 MIIz)

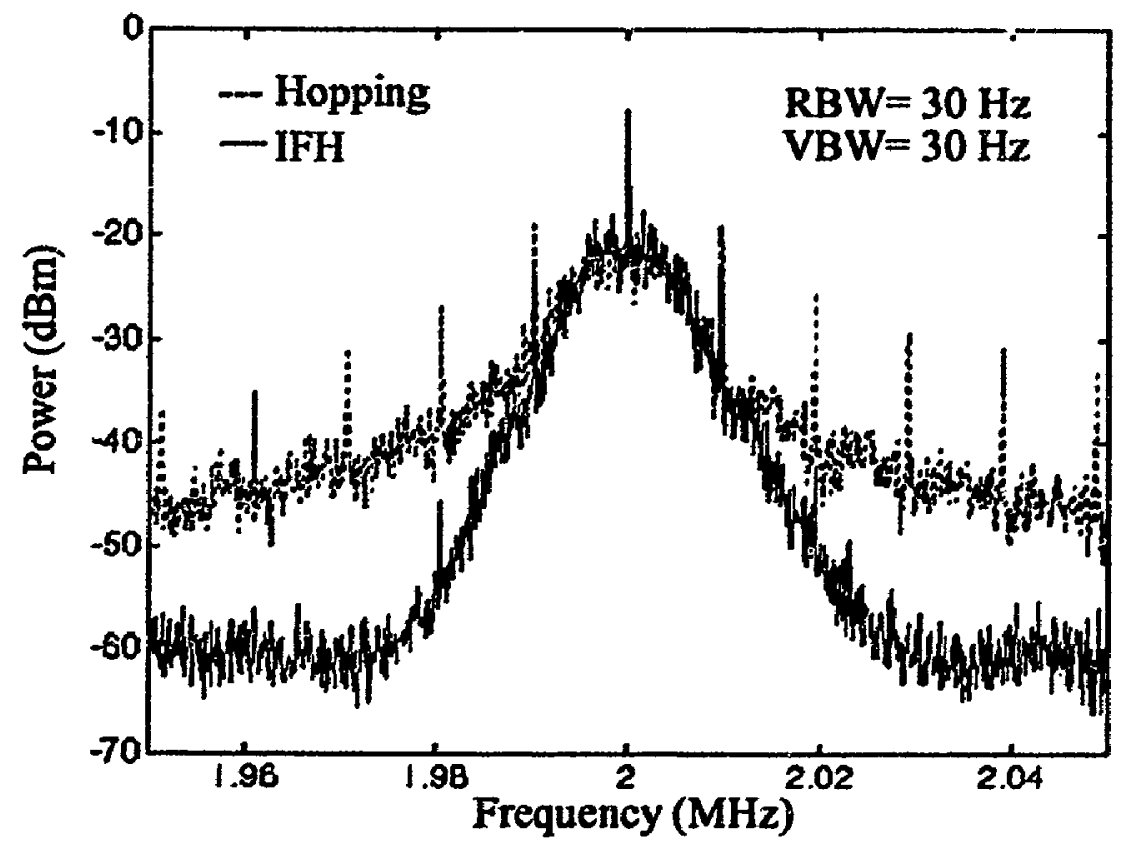


Figure 6-17: IF Spectrum (Hopping rate=10 KHz, Maximum Hop size=5.0 MHz)

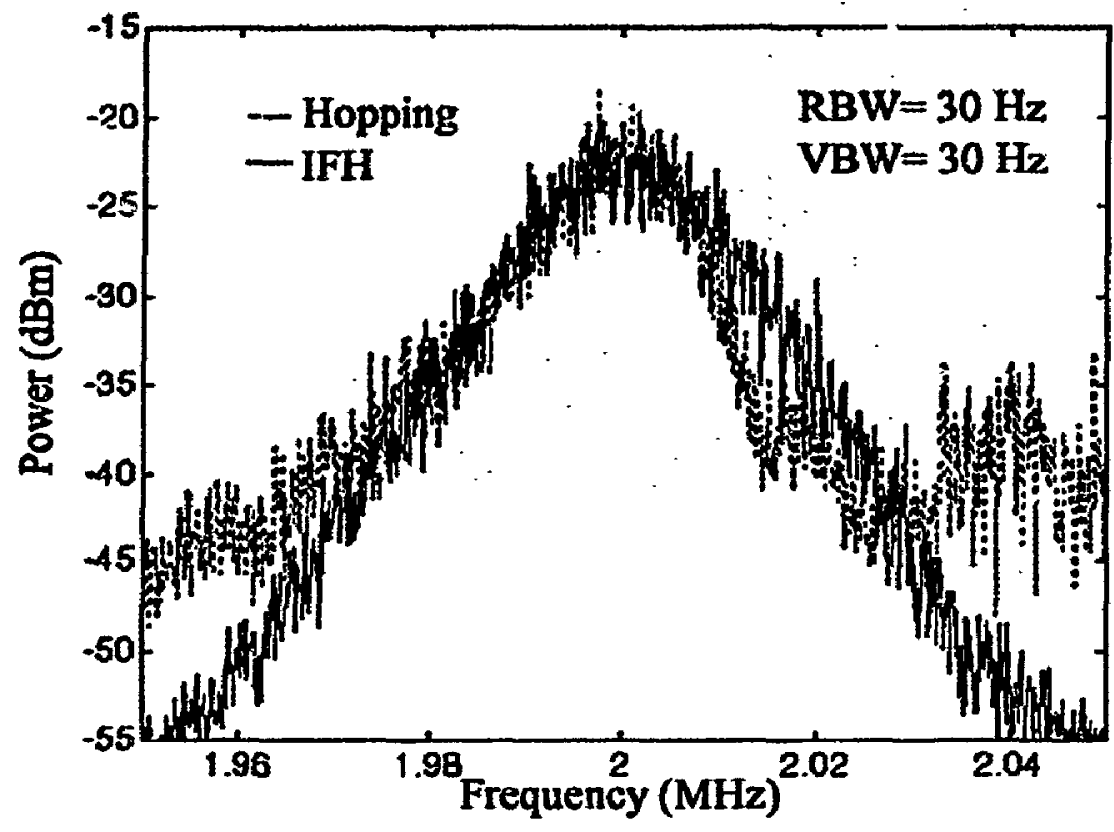

Figure 6-18: IF Spectrum (Hopping rate=78 KHz, Maximum Hop size=1.25 MHz)

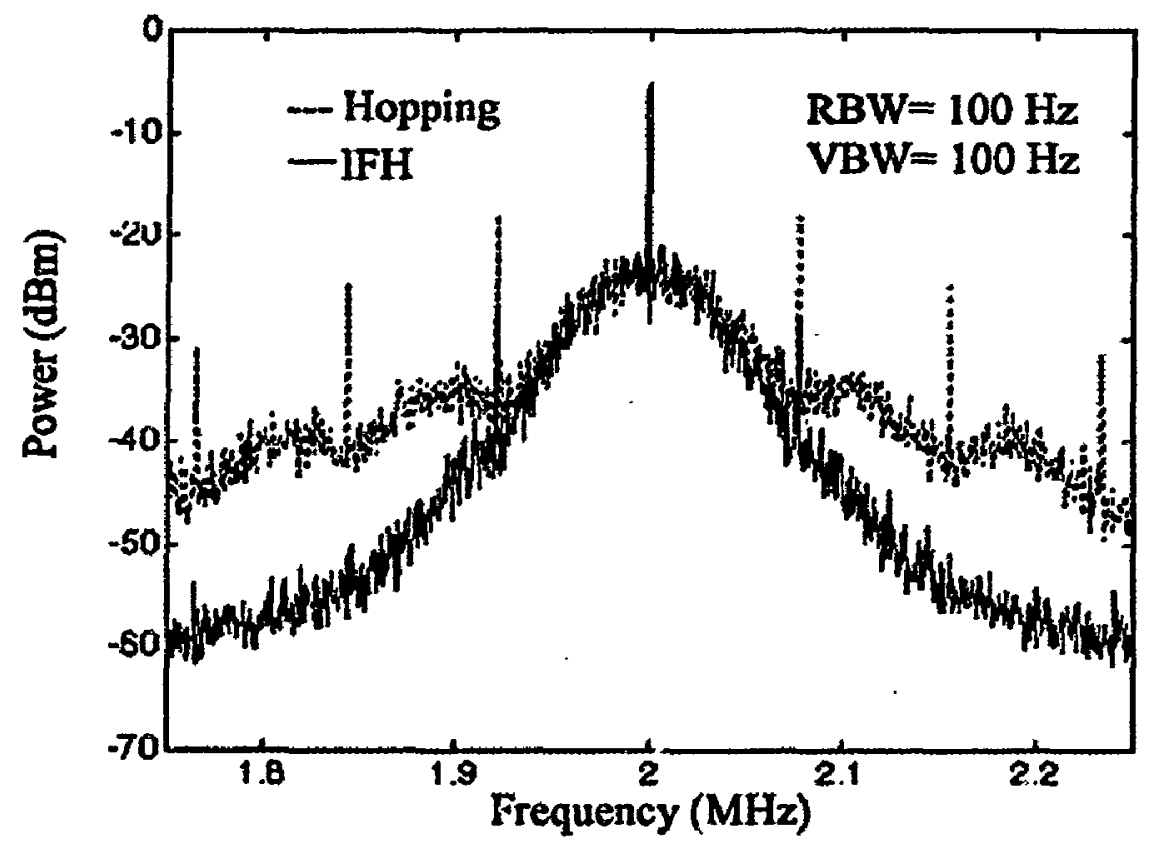


Figure 6-19: IF Spectrum (Hopping rate=78 KHz, Maximum Hop size=1.75 MHz)

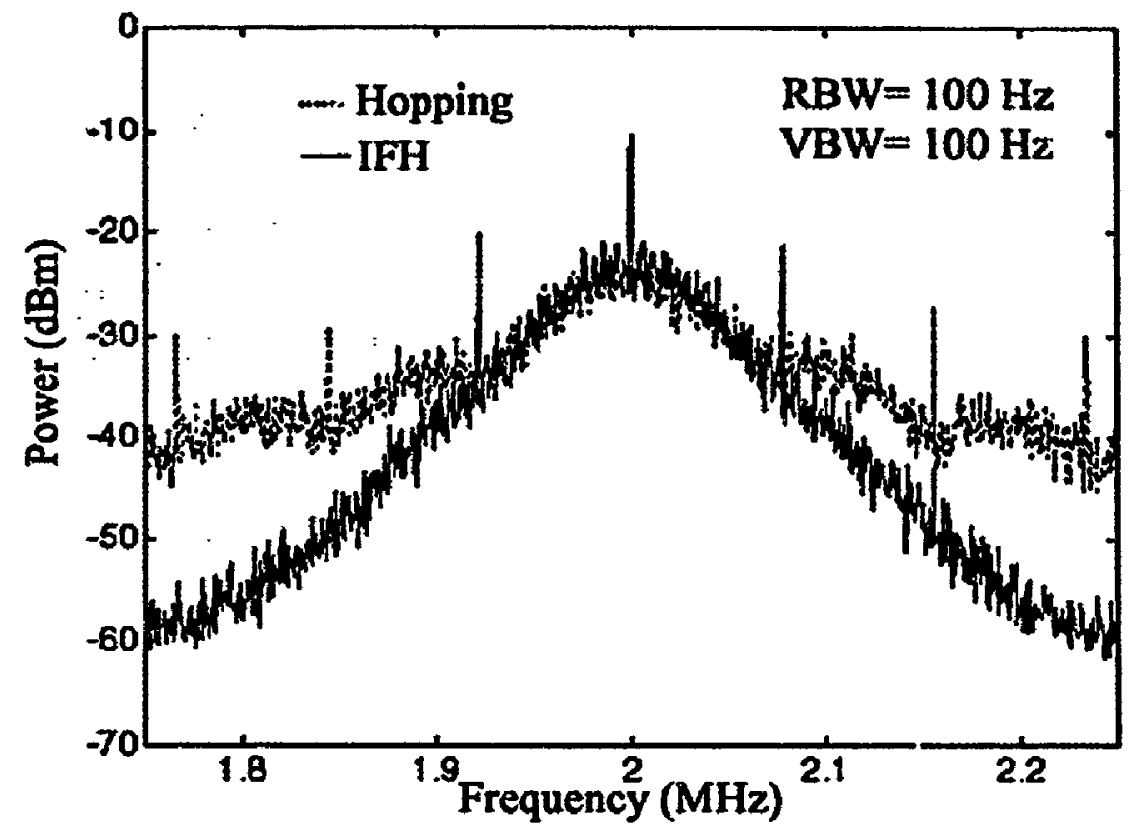

Figure 6-20: IF Spectrum (Hopping rate=7£ KHz, Maximum Hop size=2.5 MHz)

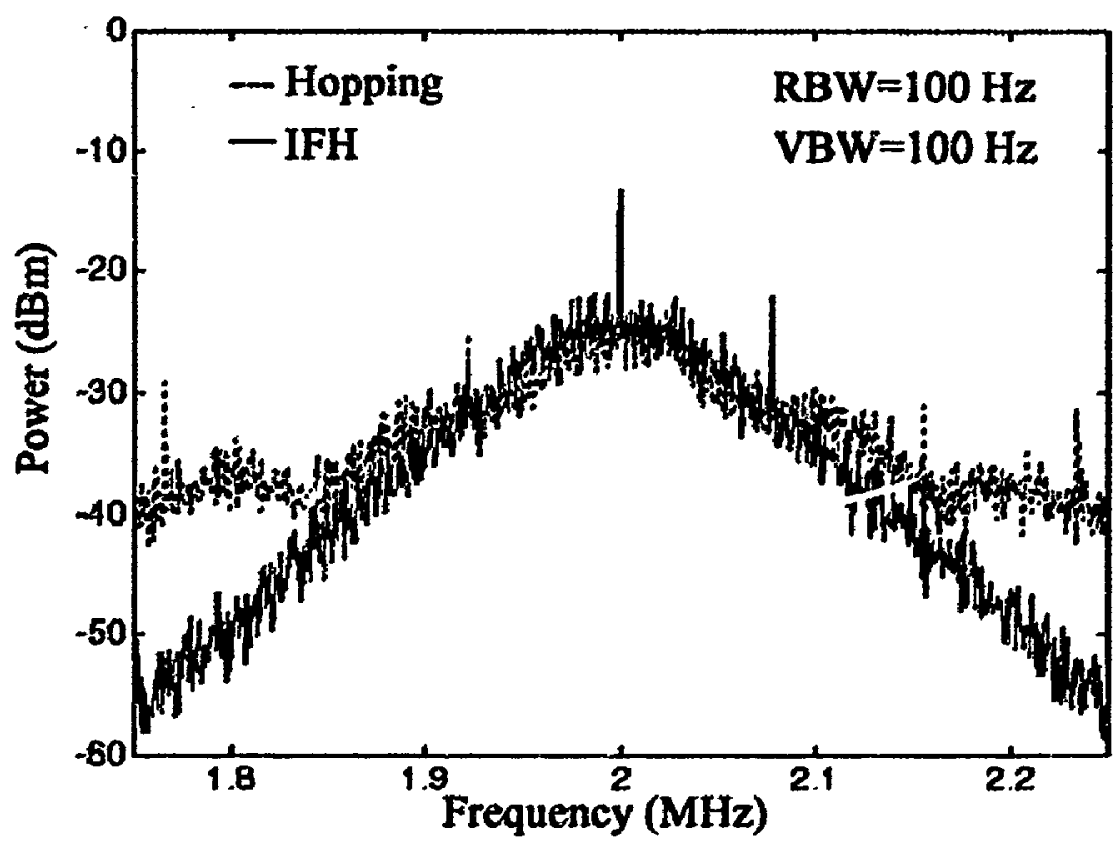


Figure 6-21: IF Spectrum (Hopping rate=78 KHz, Maximum Hop size=5.0 MHz)

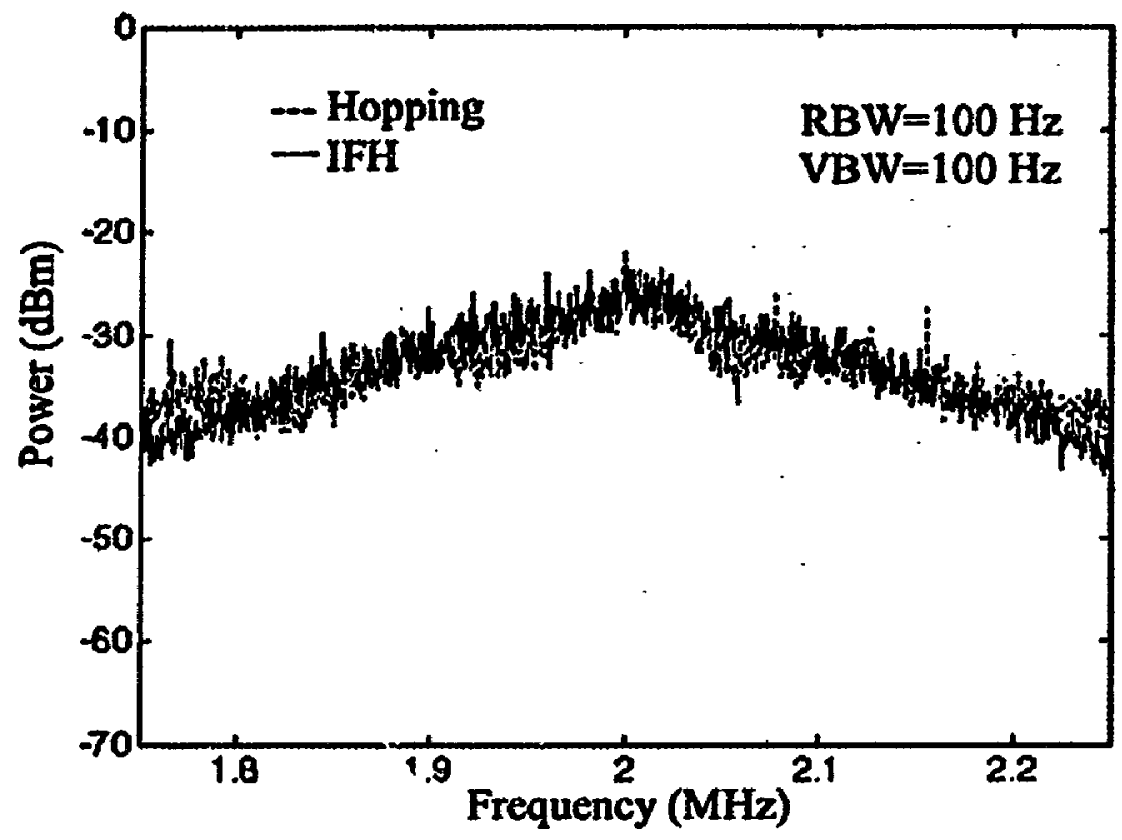

Figure б-22: IF Spectrum (Hopping rate=156 KHz, Maximum Hop size=i.25 Mî̌z)

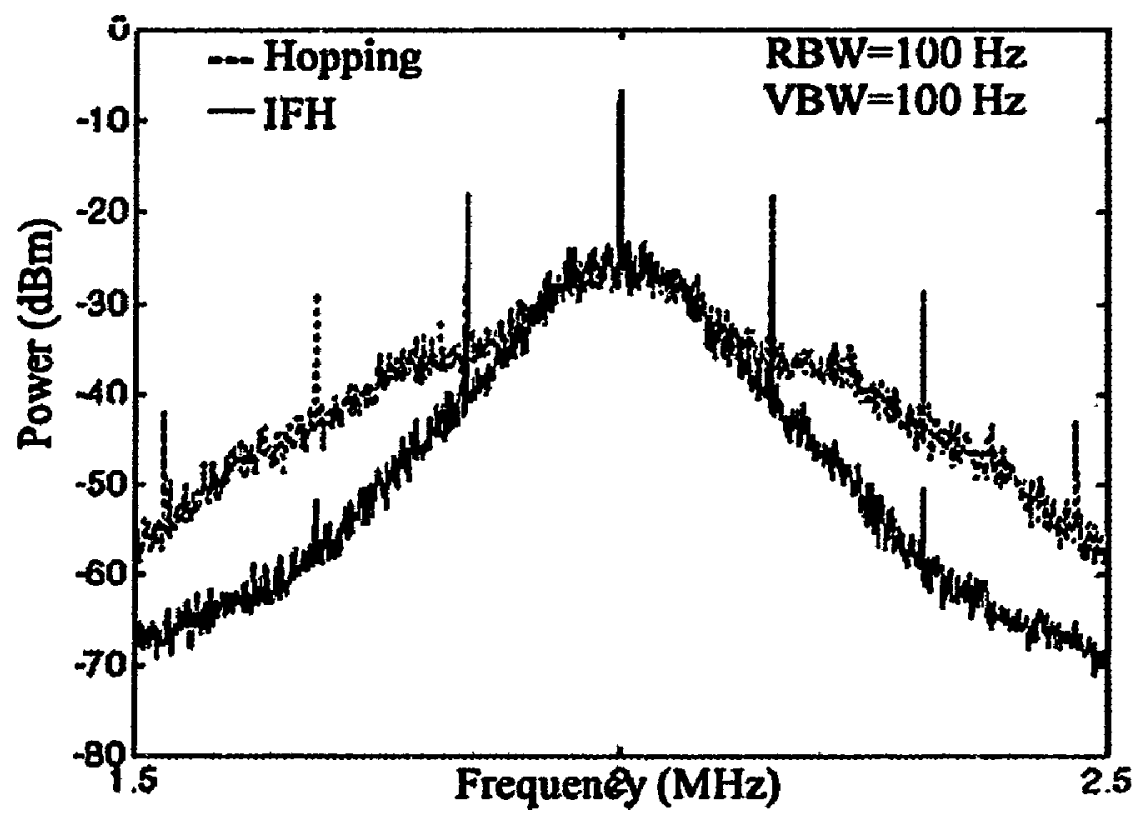


Figure 6-23: IF Spectrum (Hopping rate=156 KHz, Maximum Hop size=1.75 MHz)

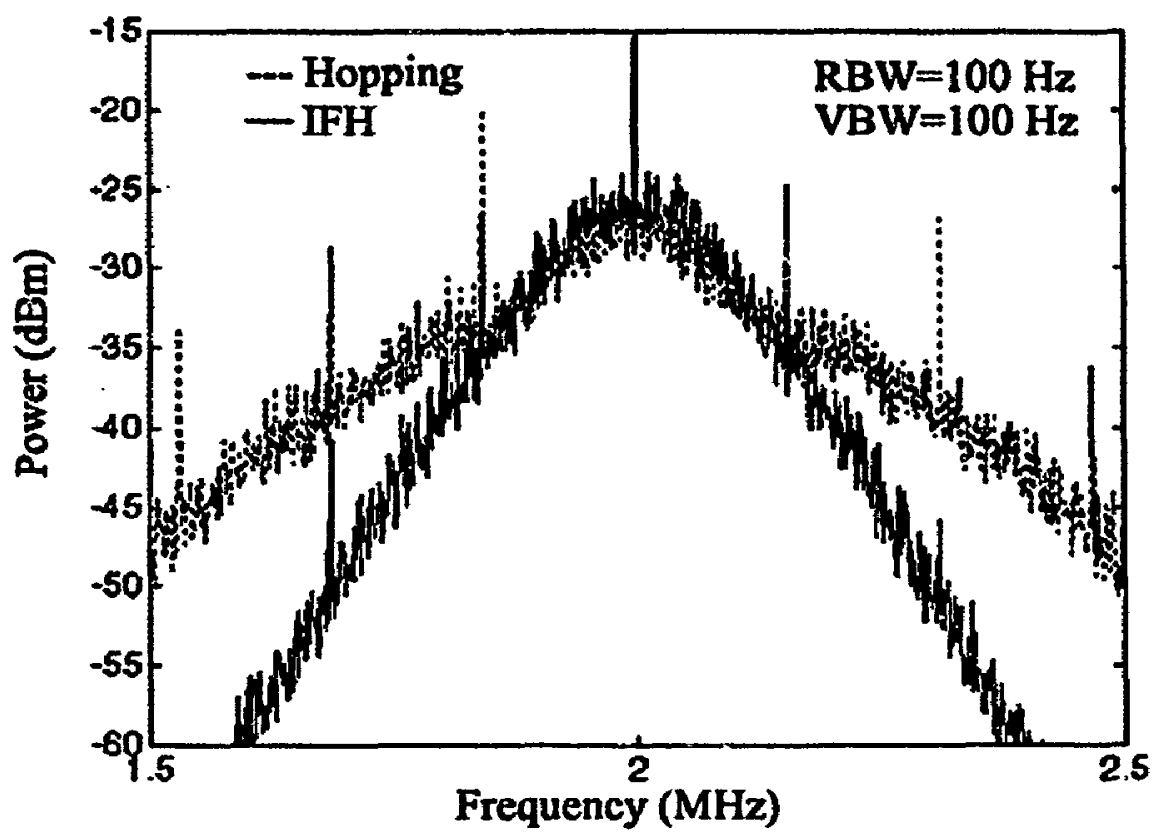

Figure 6-24: IF Spectrum (Hopping rate=156 KHz, Maximum Hop size=2.5 MHz)

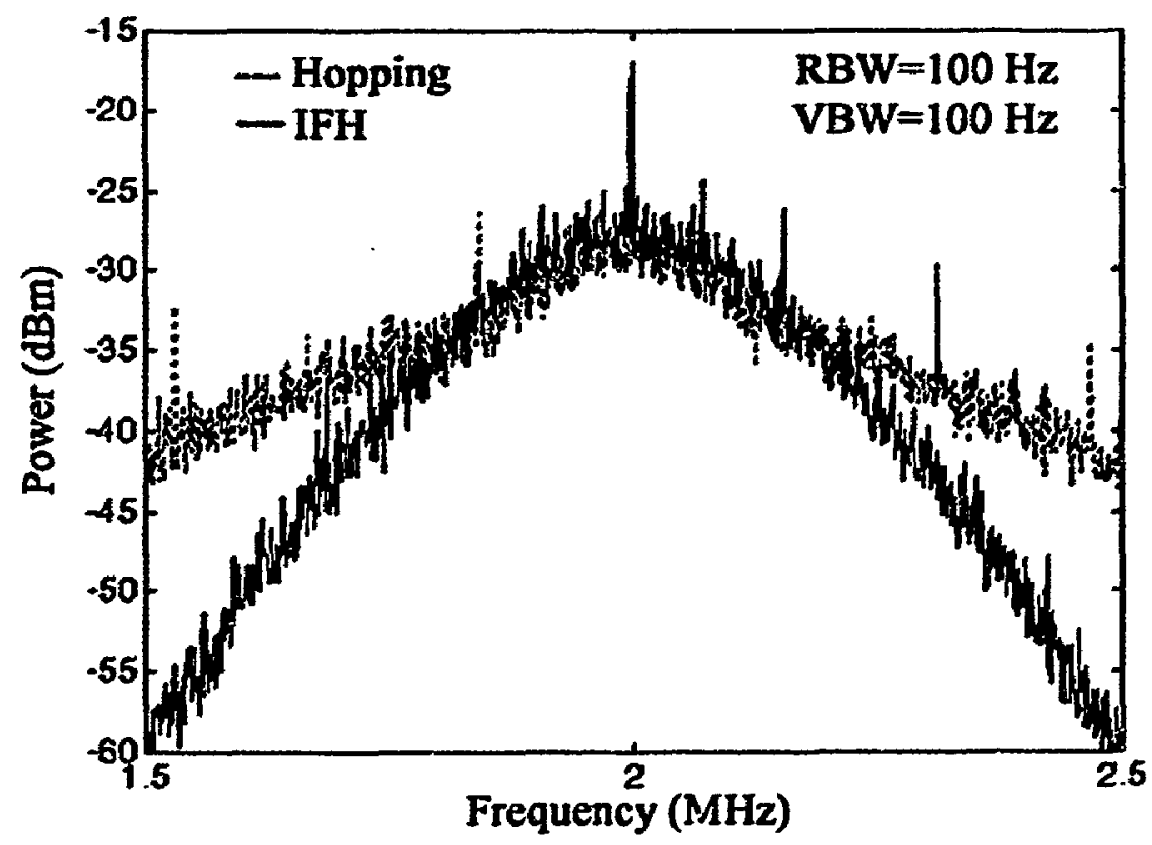


Figure 6-25: IF Spectrum (Hopping rate=156 KHz, Maximum Hop size-5.0 MHz)

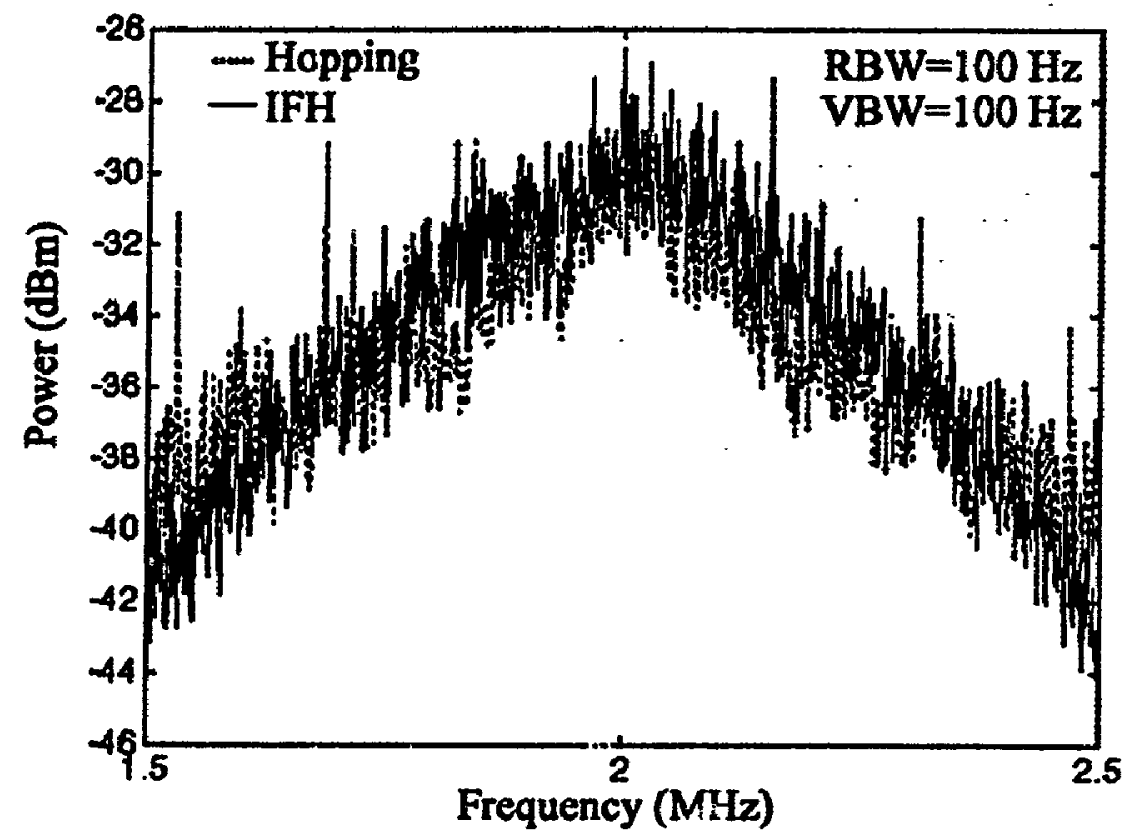

For simple frequency-hopping, it can be seen that the IF spectrums shown in Figures (6-15) to (6-25) contain a high level of phase-noise and a series of nulls and spikes, occurring regularly at spacings equal to the hopping rate, as predicted by the simulation results presented in Figures (6-5) to (6-7). It can be seen from Figures (6-15) to (6-25) that as the hopping rate is increased and/or the hop sizes are increased, the IF distortion for frequencyhopping transmission becomes more severe. The code used in the laboratory repeats every 0.1 seconds and therefore has a deterministic component present at a $10 \mathrm{~Hz}$ rate. The simple frequency-hopping IF spectrum is actually made up of discrete frequency components present at $10 \mathrm{~Hz}$ spacings, the repetition rate of the hopping code. These compoirents are not visible in Figures $(6-15)$ to $(6-25)$ because they are narrower than the resolution bandwidth of the spectrum analyzer used. The hopping codes used are slightly unbalanced (i.e., contain an unequal number of ones and zeros), resulting in the spikes present in the simulated, and measured IF spectrums.

The plots of the IF frequency spectrums measured for interpolated frequency-hopping transmission presented in Figures (6-15) to (6-25) show a sharp reduction in phase-noise when compared to the plots for simple frequency-hopping, and the nulls and spikes seen 
for simple frequency-hopping are almost completely eliminated. Because of the interpolation filter employed in interpolated frequency-hopping, the energy of the hopping code feed-through present in the IF is mostly limited to the first lote of the hopping code. When the hopping rates and hop sizes become too large for the PLLs used, very little improvement in transmission quality can be obtained through the use of interpolated frequency-hopping, as shown in Figures (6-17), (6-21), and (6-25).

Figures (6-15) to (6-17) can be compared to Figures (6-5) to (6-7) which show the simulated IF frequency spectrums for both simple frequency-hopping and interpuiated frequency-hopping obtained using MATLAB, for the same code sequence used in the laboratory measurements, and a hopping rate of $10 \mathrm{KHz}$. The results obtained in the laboratory for the $10 \mathrm{KHz}$ hopping rate are very similar to the simulation results shown in Figures (6-5) to (6-7), except for an increase in the noise floor due to added sources of noise, and otner small discrepancies. Because it is almost impossible to fully characterize the mismatch between the two PLL boards used in the laboratory, the simulation results are not completely identical to those obtained in the laboratory. Simulated performance for a maximum hop size of 5.0 $\mathrm{MHz}$ presented in Figure (6-5) is much better than the measured result presented in Figure (6-17). This is due to non-ideal limitations present in the actual system and that are not accounted for in the simulations.

The absslute received power levels for both simple frequency-hopping transmission and interpolated frequency-hopping transmission at the IF centre frequency for the different hopping rates and deviations used were measured and are summarized in Table (6-1). It is important to note that the power levels presented in Table (6-1) were measured with a spectrum analyzer and are average power measurements as opposed to peak power measurements. 
Table 6-1: Absolute Received Power

\begin{tabular}{|c|c|c|c|}
\hline Hopping Rate and Maximum Hop Size & $\begin{array}{c}\text { Power } \\
\text { (dBm) } \\
\text { Hopping }\end{array}$ & $\begin{array}{c}\text { Power } \\
\text { (dBm) } \\
\text { IFH }\end{array}$ & $\begin{array}{l}\text { Śpectral- } \\
\text { Splatter } \\
\text { Reduction } \\
\text { (dB) }\end{array}$ \\
\hline Rate $=10 \mathrm{KHz}$, Hop Size $=1.25 \mathrm{MHz}$ & -5.4 & -3.3 & 2.1 \\
\hline Rat $=10 \mathrm{KHz}$, Hop Size $=2.5 \mathrm{MHz}$ & -13.9 & -8.7 & 5.2 \\
\hline Rate $=10 \mathrm{KHz}$, Hop Size $=5.0 \mathrm{MHz}$ & -22.5 & -26.8 & -4.3 \\
\hline Rate $=78 \mathrm{KHz}$, Hop Size $=1.25 \mathrm{MHz}$ & -12.4 & -7.5 & 4.9 \\
\hline Rate $=78 \mathrm{KHz}$, Hop Size $=1.75 \mathrm{MHz}$ & -19.7 & -12.6 & 7.1 \\
\hline Rate $=78 \mathrm{KHz}$, Hop Size $=2.5 \mathrm{MHz}$ & -23.4 & -17.9 & 5.5 \\
\hline Rate $=78 \mathrm{KHz}$, Hop Size $=5.0 \mathrm{MHz}$ & -26.9 & -27.3 & -0.4 \\
\hline Rate $=156 \mathrm{KHz}$, Hop Size $=1.25 \mathrm{MHz}$ & -14.9 & -9.2 & 5.7 \\
\hline Rate $=156 \mathrm{KHz}$, Hop Size $=1.75 \mathrm{MHz}$ & -23.3 & -15.9 & 7.4 \\
\hline Rate $=156 \mathrm{KHz}$, Hop Size $=2.5 \mathrm{MHz}$ & -28.2 & -20.4 & 7.85 \\
\hline Rate $=156 \mathrm{KHz}$, Hop Size $=5.0 \mathrm{MHz}$ & -41.5 & -29.0 & 12.5 \\
\hline
\end{tabular}

The results presented in Table (6-1) show that significant amounts of transmitter output power are lost due to spectral-splatter when using standard frequency-hopping, when compared with the stationary carrier case shown in Figure (6-12). These results show a significant average reduction in spectral-splatter for interpolated frequency-hopping over simple frequency-hopping, especially for higher hopping rates and frequency deviations, except when the phase-error becomes excessive and the difference in average received power becomes negligible. At the sampling instant, the peak power at the IF centre frequency for simple frequency-hopping should be as high, or higher, than that received for interpolated frequency-hopping. Therefore, the results presented in Table (6-1) are not neccessarily an indication of the achievable bit-error rate for simple-frequency-hopping but rather are an indication of the amount of spectral-splatter generated. For interpolated frequency-hopping, the peak power received is much more constant, and therefore the data can be sampled continuously, improving the bit-error rate performance of the receiver if an 
appropriate demodulator is employed.

The measurement results obtained for the received IF of the system seem to indicate that except for normal component variations, the two synthesizers used in the laboratory are fairly well matched. As predicted, interpolated frequency-hopping significantly reduces the spectral-splatter and IF distortion present in simple frequency-hopping, especially for hopping rates that begin to approach or exceed the PLL synthesizer corner frequency, where the effects of mismatch are greatly increased. There is a point however, where the phase-error becomes excessive for the system used, and neither form of transmission provides a significant advantage.

\subsubsection{Measurements with Data}

The system was tested with binary-FSK data modulation at a symbol rate of $78 \mathrm{~Kb} / \mathrm{s}$, and frequensy deviations of $+/-500 \mathrm{KHz}$. This was done with the same test system shown in Figure (5-1), with random data generated in software. For the purposes of simplifying the Inatched filter required in the receiver for optimal symbol detection, data pulse shaping was not used. This allowed an integrate and dump filter to be used in the FSK discriminator circuit. The eye diagrams obtained for a $10 \mathrm{KHz}$ hopping rate, a $78 \mathrm{KHz}$ hopping rate, and a $156 \mathrm{KHz}$ hopping rate are presented in Figures (6-26) to (6-37) for different maximum hop sizes. Eye diagrams provide a qualitative indication of the quality of the demodulated data bits in the receiver. The larger the eye opening is at the instant or period in which a bit decision is made, the higher the probability that the decision will be correct. 
Figure 6-26: Eye Diagram for Simple Frequency-hopping (Hopping rate=10 KHz, Maximum Hop Size=2.5 MHz)

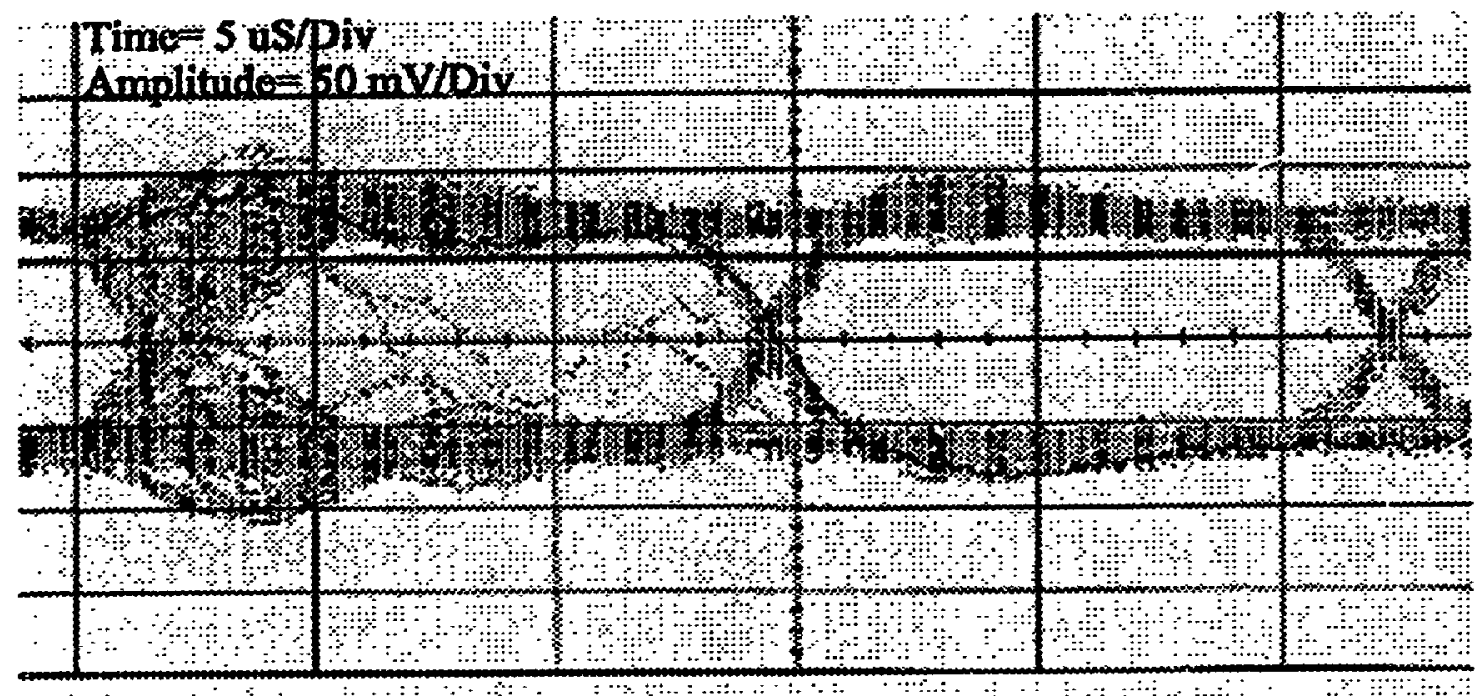

Figure 6-27: Eye Diagram for Interpolated Frequency-hopping (Hopping Rate=10 KHz, Maximum Hop Size=2.5 MHz)

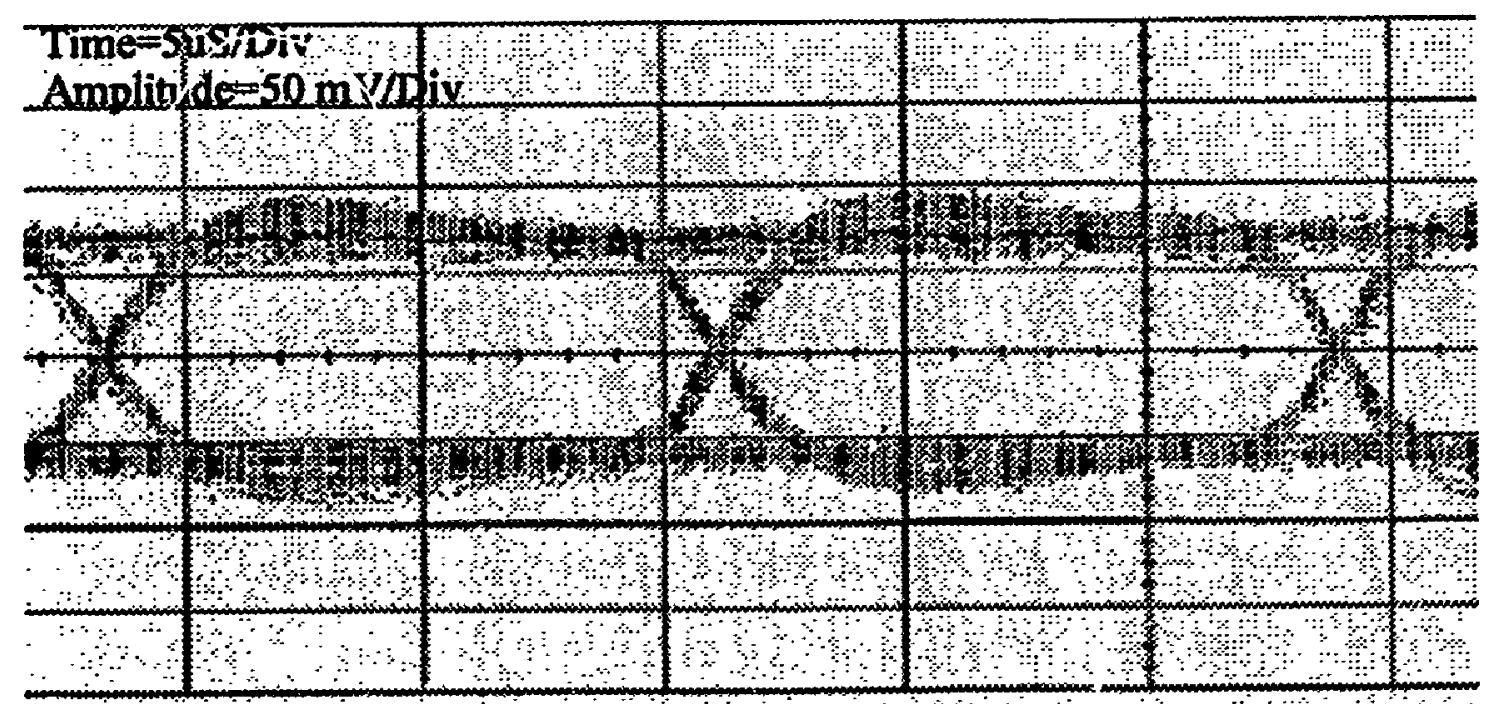


Figure 6-28: Eye Diagram for Simple Frequency-hopping (Hopping Rate $=10 \mathrm{KHz}$, Maximum Hop Sire $=5.0 \mathrm{MHz}$ )

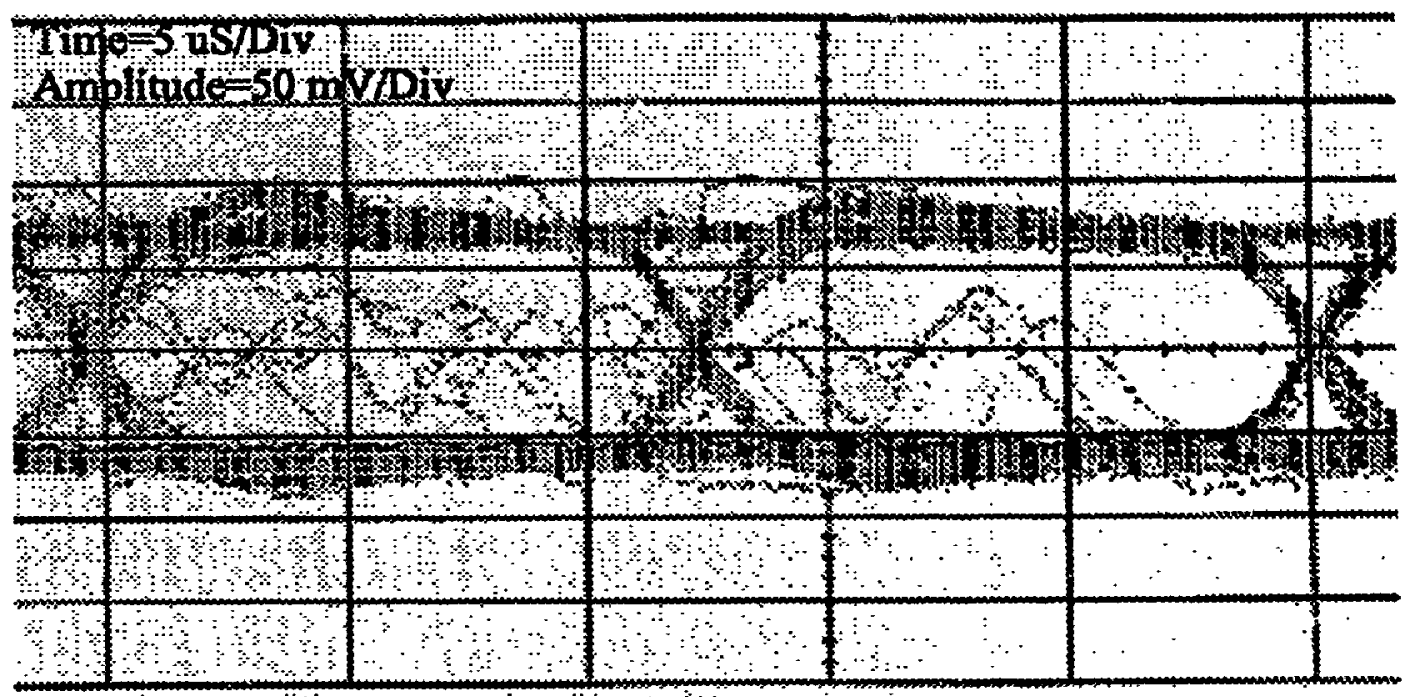

Figure 6-29: Eye Diagram for Interpolated Frequency-hopping (Hopping Rate $=10 \mathrm{KHz}$, Maximum Hop Size=5.0 MHz)

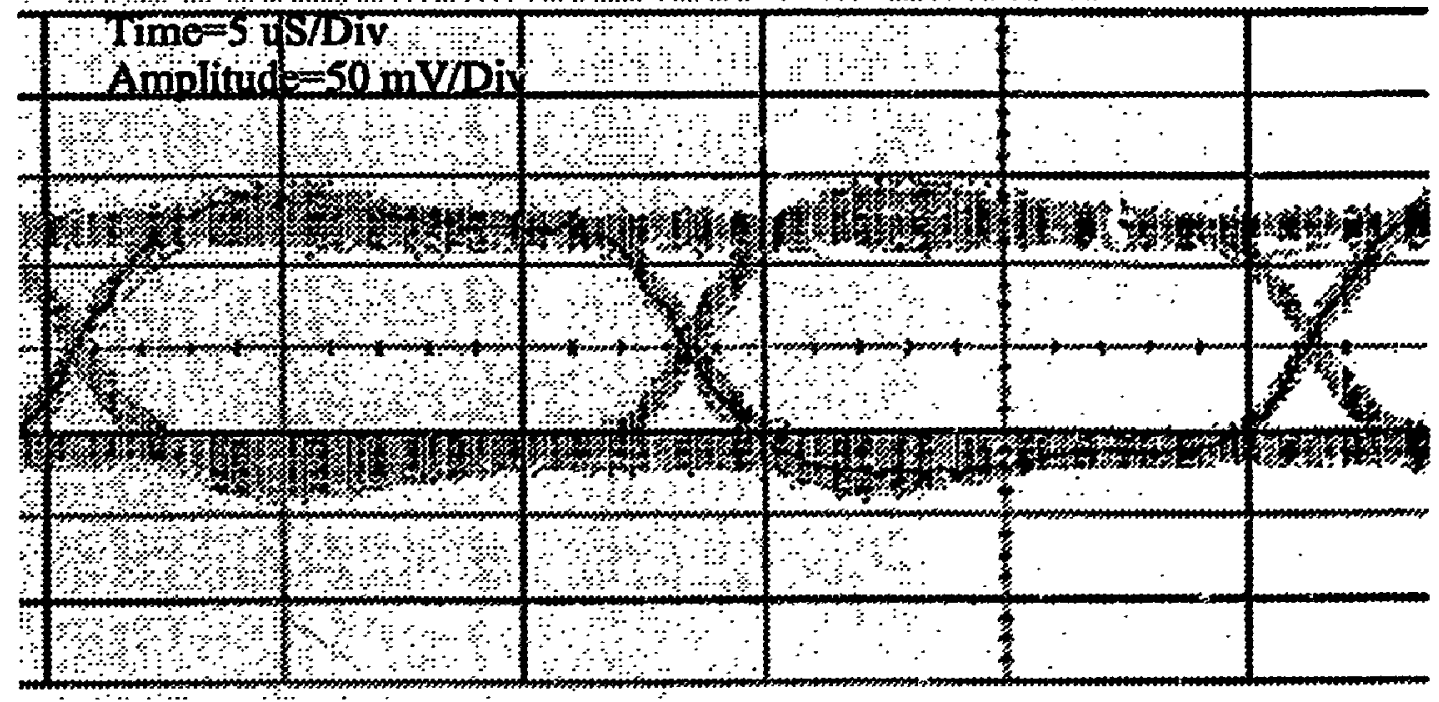


Figure 6-30: Eye Diagram for Simple Frequency-hopping (Hopping Rate=78 KHz, Maximum Hop Size=2.5 MHz)

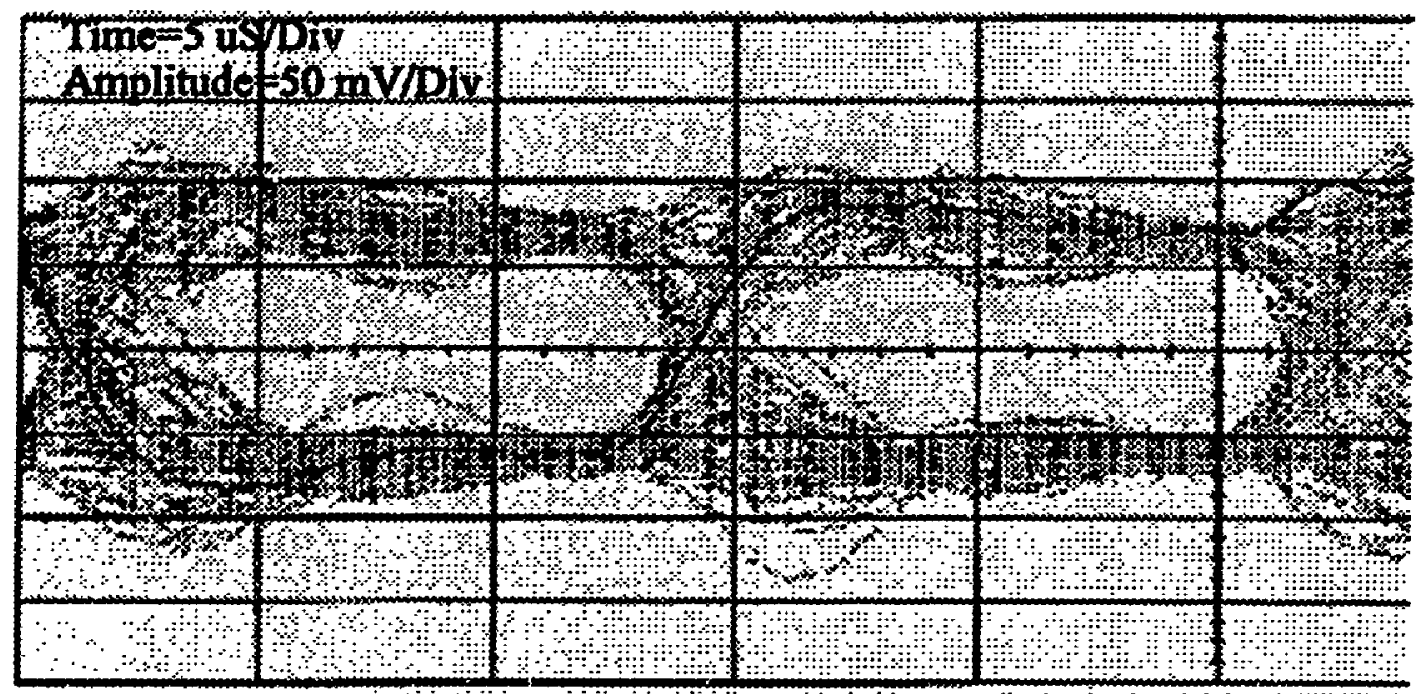

Figure 6-31: Eye Diagram for Interpolated Frequency-hopping (Hopping Rate=78 KHz, Maximum Hop Size=2.5 MHz)

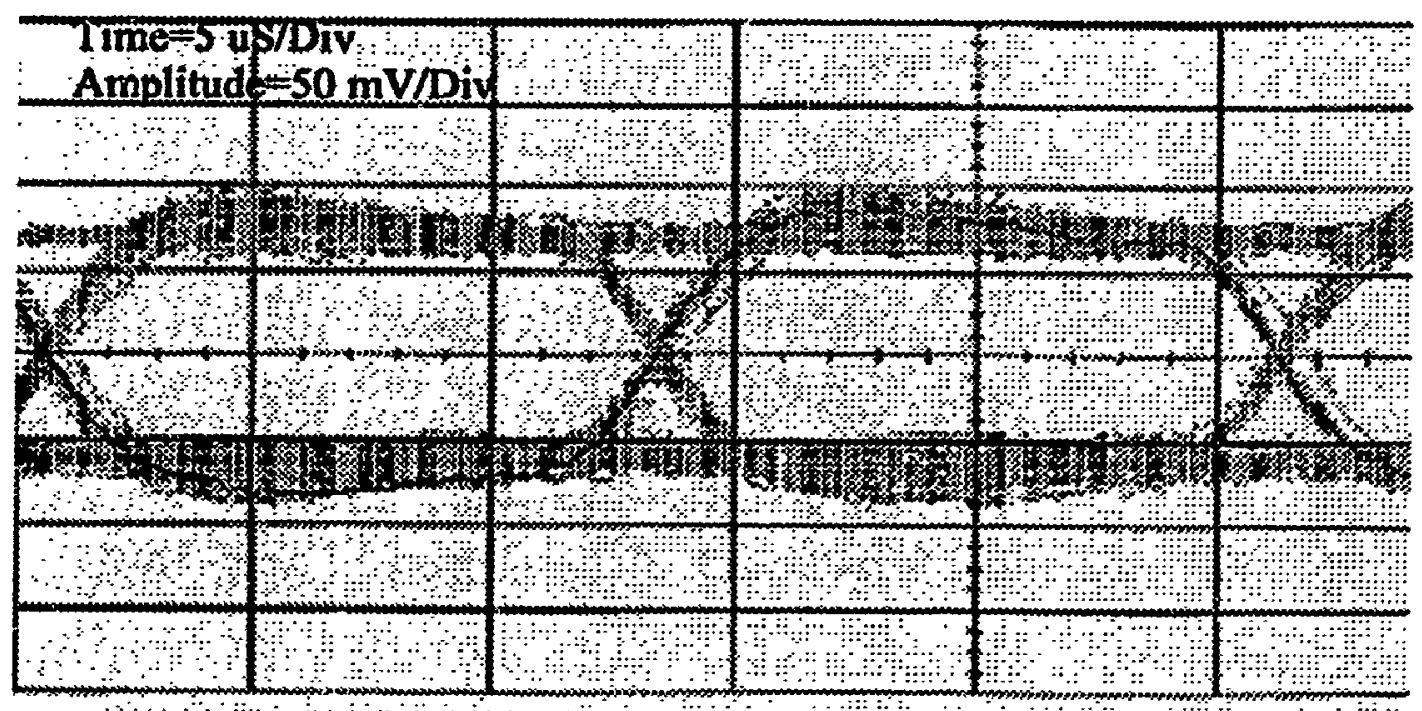




\section{Figure 6-32: Eye Diagram for Simple Frequency-hopping}

\section{(Hopping Rate=78 KHz, Maximum Hop Size $=5.0 \mathrm{MHz}$ )}

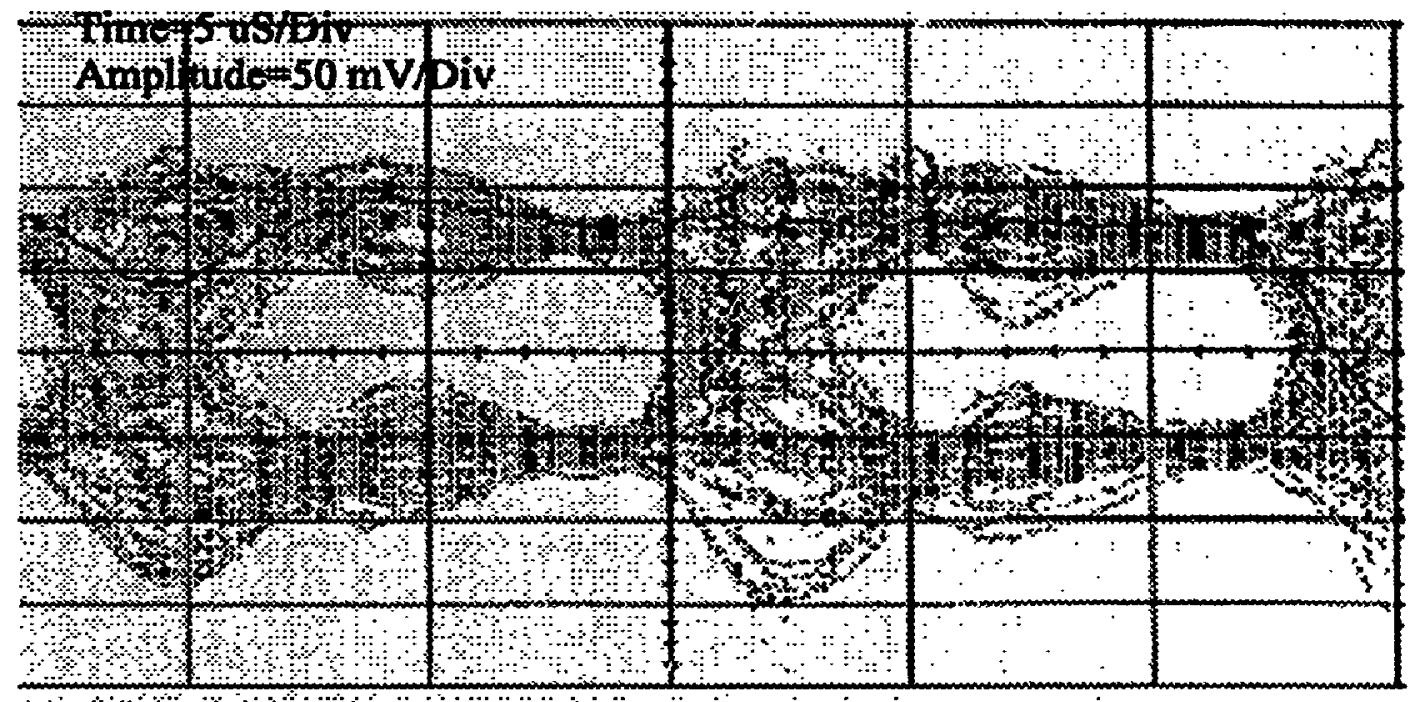

Figure 6-33: Eye Diagram for Interpolated Frequency-hopping

\section{(Hopping Rate $=78 \mathrm{KHz}$, Maximum Hop Size $=5.0 \mathrm{MHz}$ )}

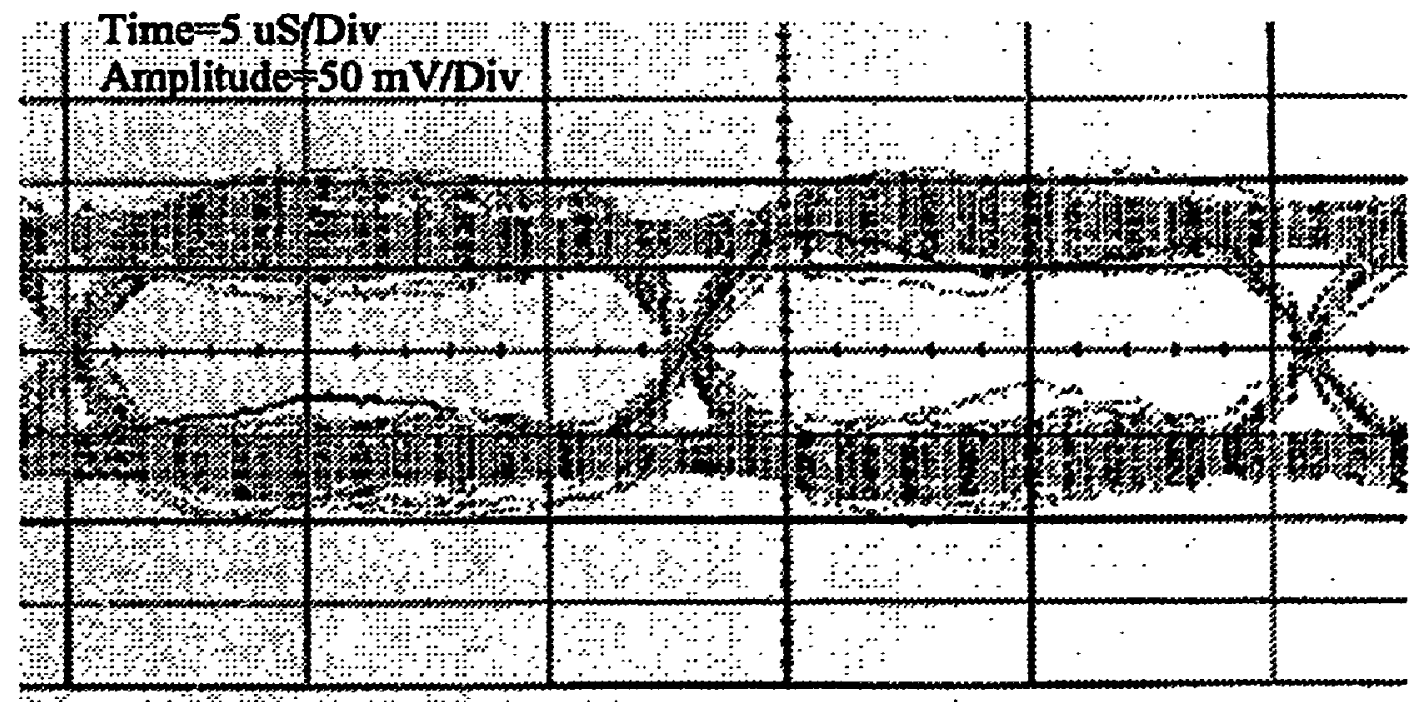




\section{Figure 6-34: Eye Diagram for Simple Frequency-hopping}

(Hopping Rate=156 KHz, Maximum Hop Size=2.5 MHz)

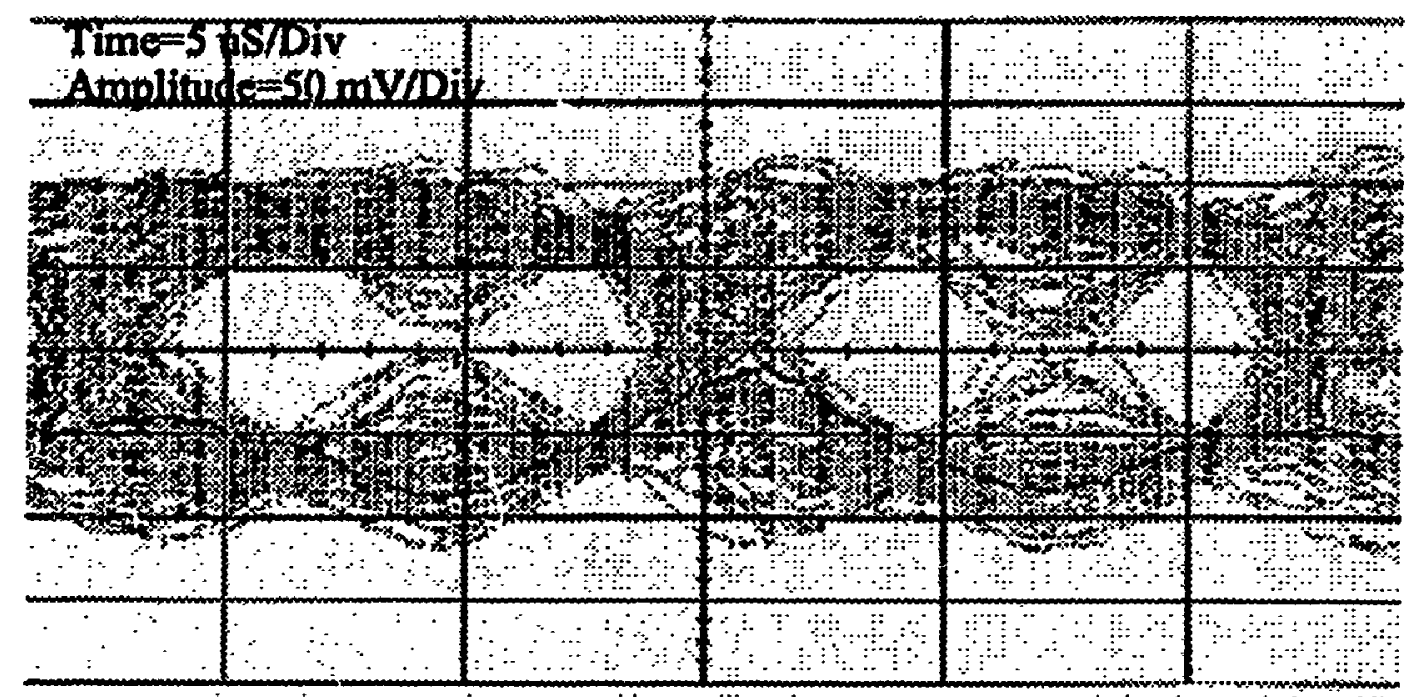

Figure 6-35: Eye Diagram for Interpolated Frequency-hopping (Hopping Rate=156 KHz, Maximum Hop Size=2.5 MHz)

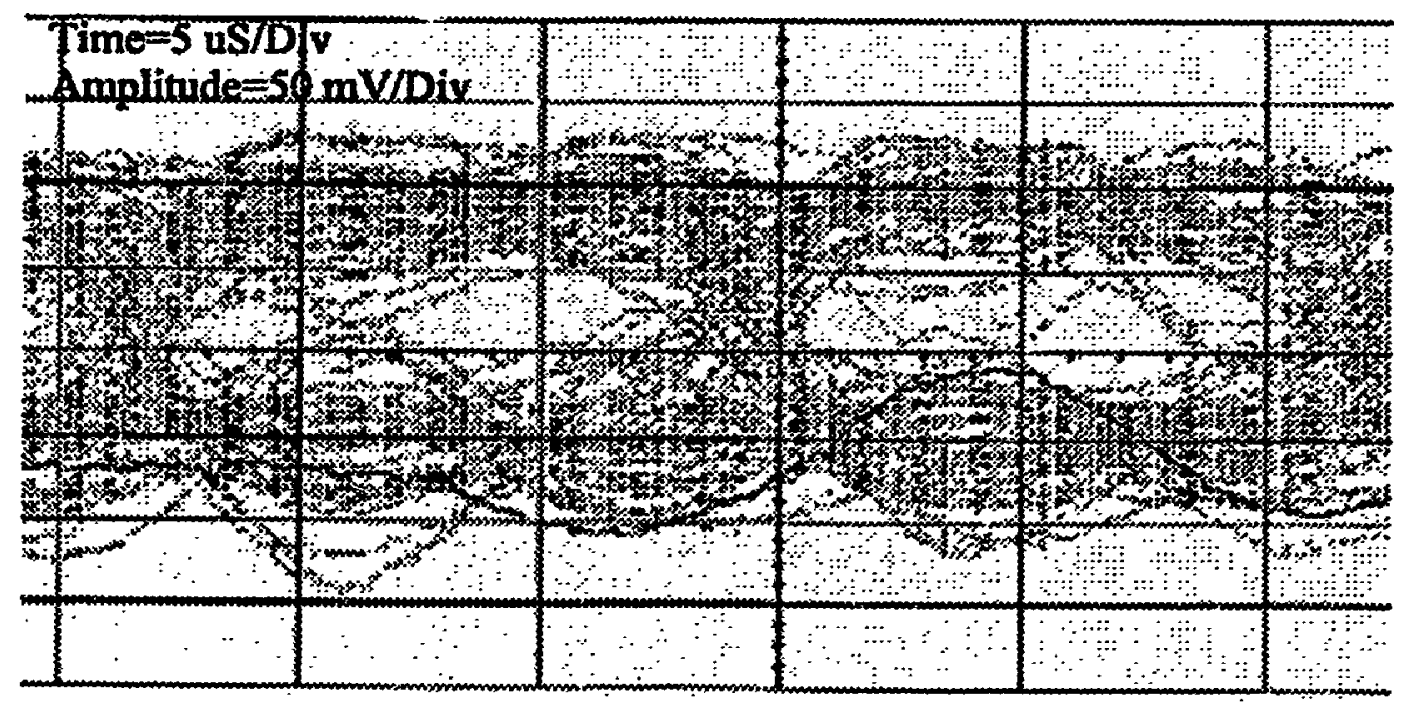


Figure 6-36: Eye Diagram for Simple Frequency-hopping (Hopping Rate=156 KHz, Maximum Hop Size=5.0 MHz)

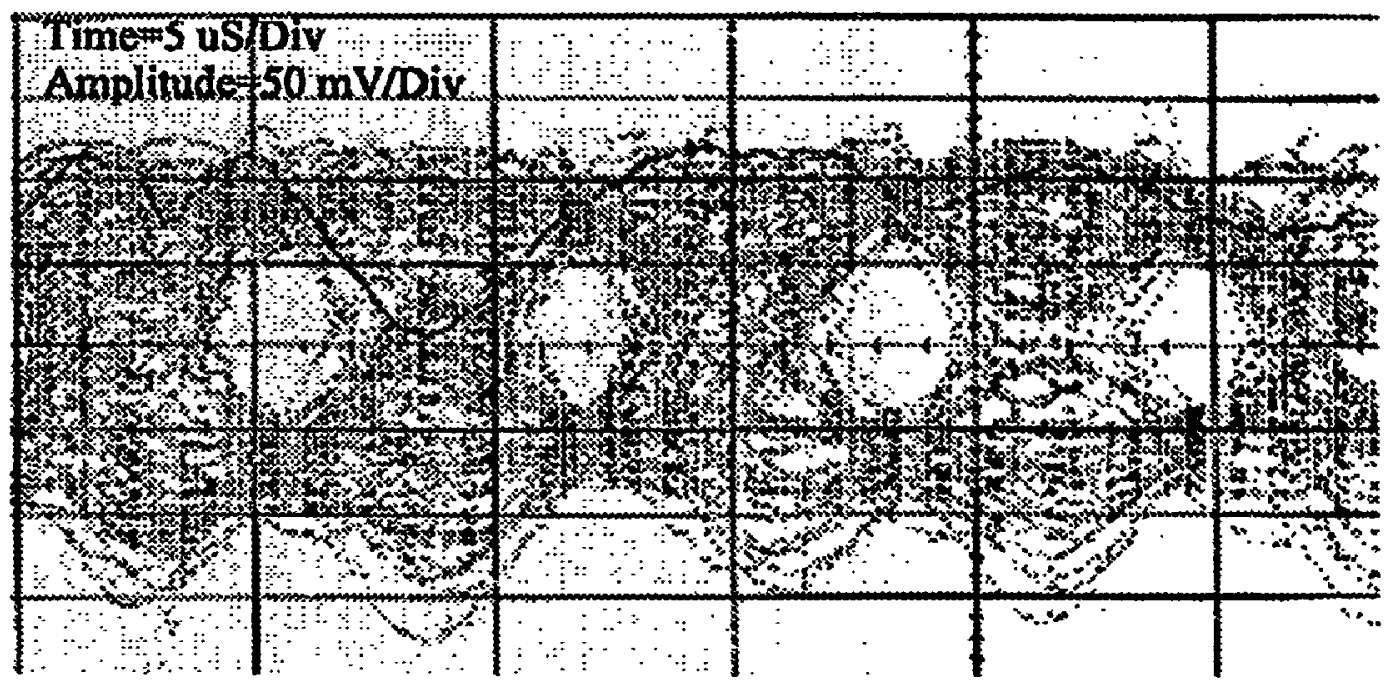

Figure 6-37: Eye Diagram for Interpolated Frequency-hopping (Hopping Rate=156 KHz, Maximum Hop Size=5.0 MHz)

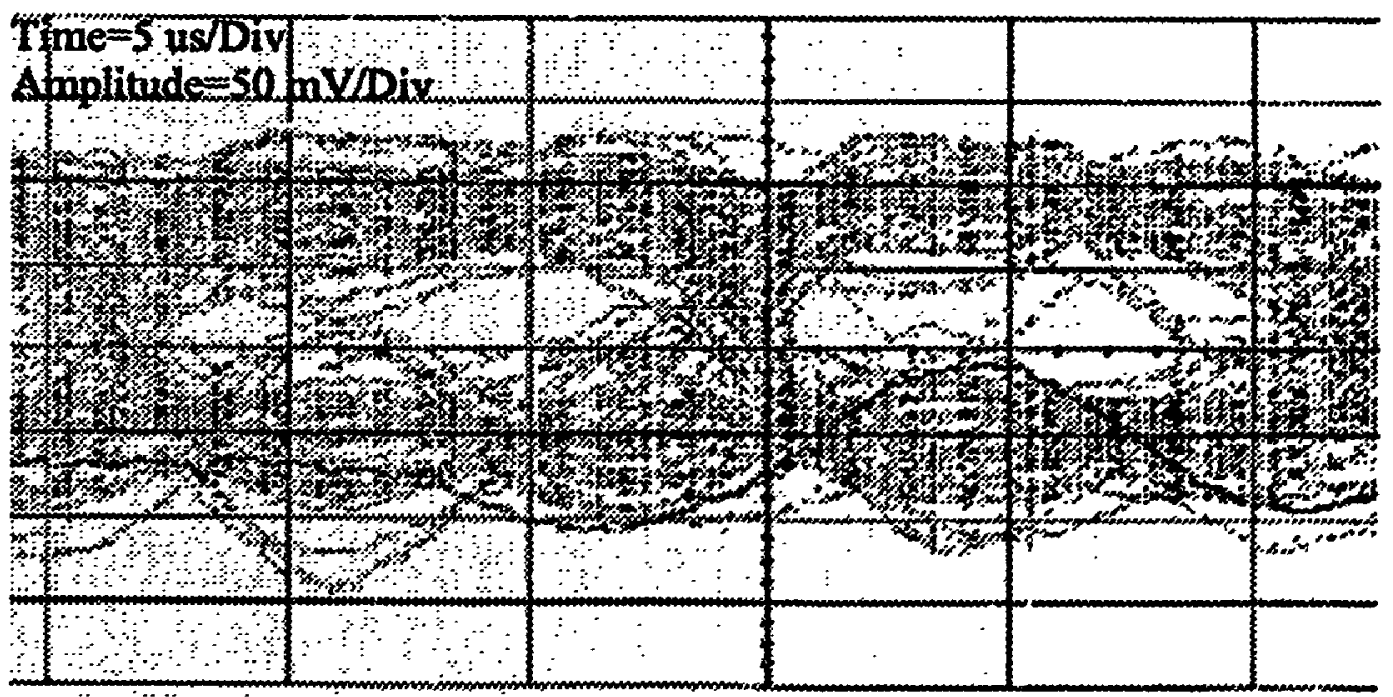

For the $10 \mathrm{KIYz}$ hopping rate, the transmission is slow frequency-hopping and there are eight data symbols transmitted per frequency hop. The hopping transients present in slow, simple frequency-hopping transmission can clearly be seen in Figures (6-26), (6-28), and (6-30). For the $78 \mathrm{KHz}$ and $156 \mathrm{KHz}$ hopping rates, the transmission is fast frequency- 
hopping with one and two hops per transmitted data symbol respectively. It can ve seen from Figures (6-26), (6-28), (6-30), (6-32), (6-34) and (6-36) that as the hopping rate and the maximum hop sizes increase, the eye diagrams for simple frequency-hopping become distorted, especially at the transition edges, with overshoot and ringing clearly seen in the diagrams. For the $156 \mathrm{KHz}$ hopping rate, as there are two frequency hops per transmitted data symbol, the centre of the eye is almost completely closed for the simple frequency hopping cases. The eye diagrams for interpolated frequency-hopping transmission shown in Figures (6-27), (6-29), (6-31), (6-33), (6-35), and (6-37) become more distorted as the hopping rate and the maximum hop sizes are increased. The transition edges show very little distortion, except when the phase-error becomes too excessive for the system used. The distortion present in the eye diagrams is consistent, and does not take the form of transients as in the standard frequency-hopping case. This means that as the hopping rates and the maximum hop sizes increase, the bit-error rate for interpolated frequency-hopping transmission should be lower than that obtained for simple frequency-hopping transmission at the same rate.

Figures (6-38) to (6-43) show plots of bit-error rate vs. Eb/No for both simple frequencyhopping and interpolated frequency-hopping for a $10 \mathrm{KHz}$ hopping rate, a $78 \mathrm{KHz}$ hopping rate, and a $156 \mathrm{KHz}$ hopping rate. Two scales are given for the horizontal axis, the first being noise attenuation in decibels (refer to Figure (5-1)), and the second being transmitted $\mathrm{Eb} /$ No in decibels for the test system used. The step attenuators used have a worst case accuracy of $+/-1 \mathrm{~dB}$, whereas the Eb/No measurements where made using a spectrum analyzer and have higher measurement error. Therefore, an $\mathrm{Eb} / \mathrm{No}$ measurement was made for each form of transmission at an attenuator setting of zero, and used as an absolute reference level. The purpose of the bit-error rate measurements made was not to compare the transmission performance of standard frequency-hopping and interpolated frequencyhopping transmission to the ideal non-coherent FSK case, but rather to compare the various transmission methods used to each other. For this purpose, measurements were also made for the stationary case in order to show that some of the transmission limitations encountered were due to the test system used, rather than the transmission method. 
Figure 6-38: Bit-error Rate vs. Eb/No for Simple Frequency-hopping at $10 \mathrm{KHz}$

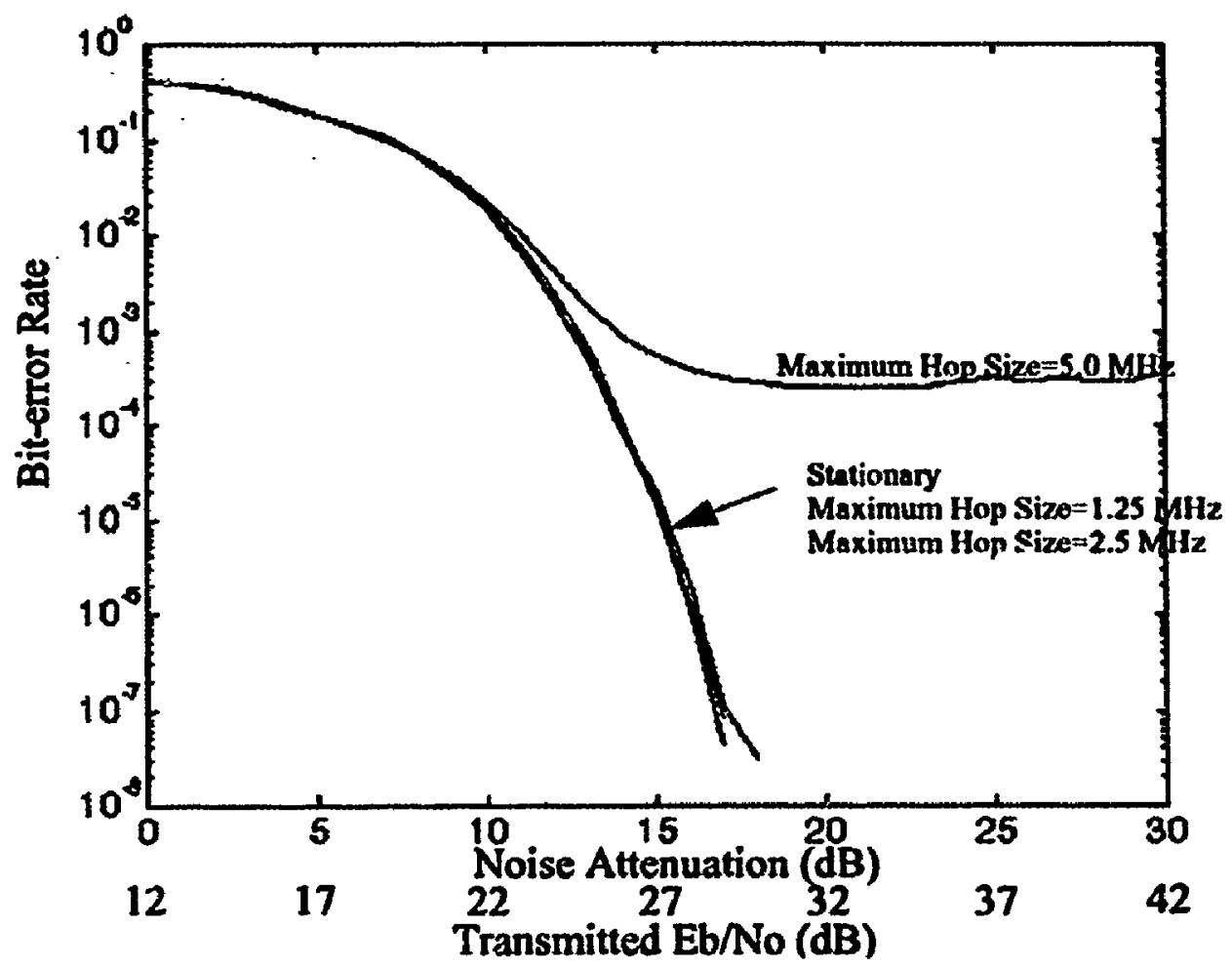

Figure 6-39: Bit-error Rate vs. Eb/No for Interpolated Frequency-hopping at $10 \mathrm{KHz}$

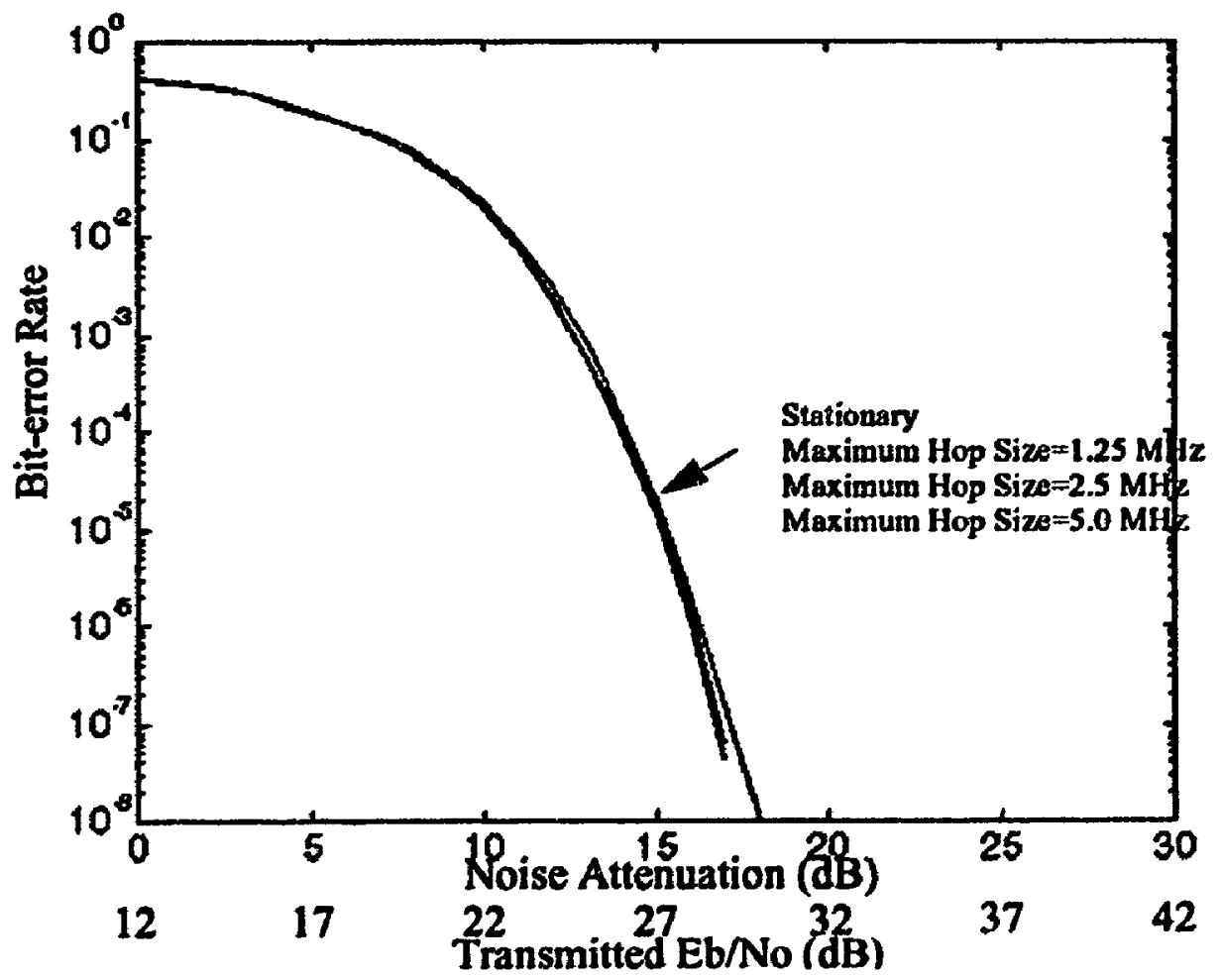


Figure 6-40: Bit-error Rate vs. Eb/No for Simple Frequency-hopping at $78 \mathrm{KHz}$

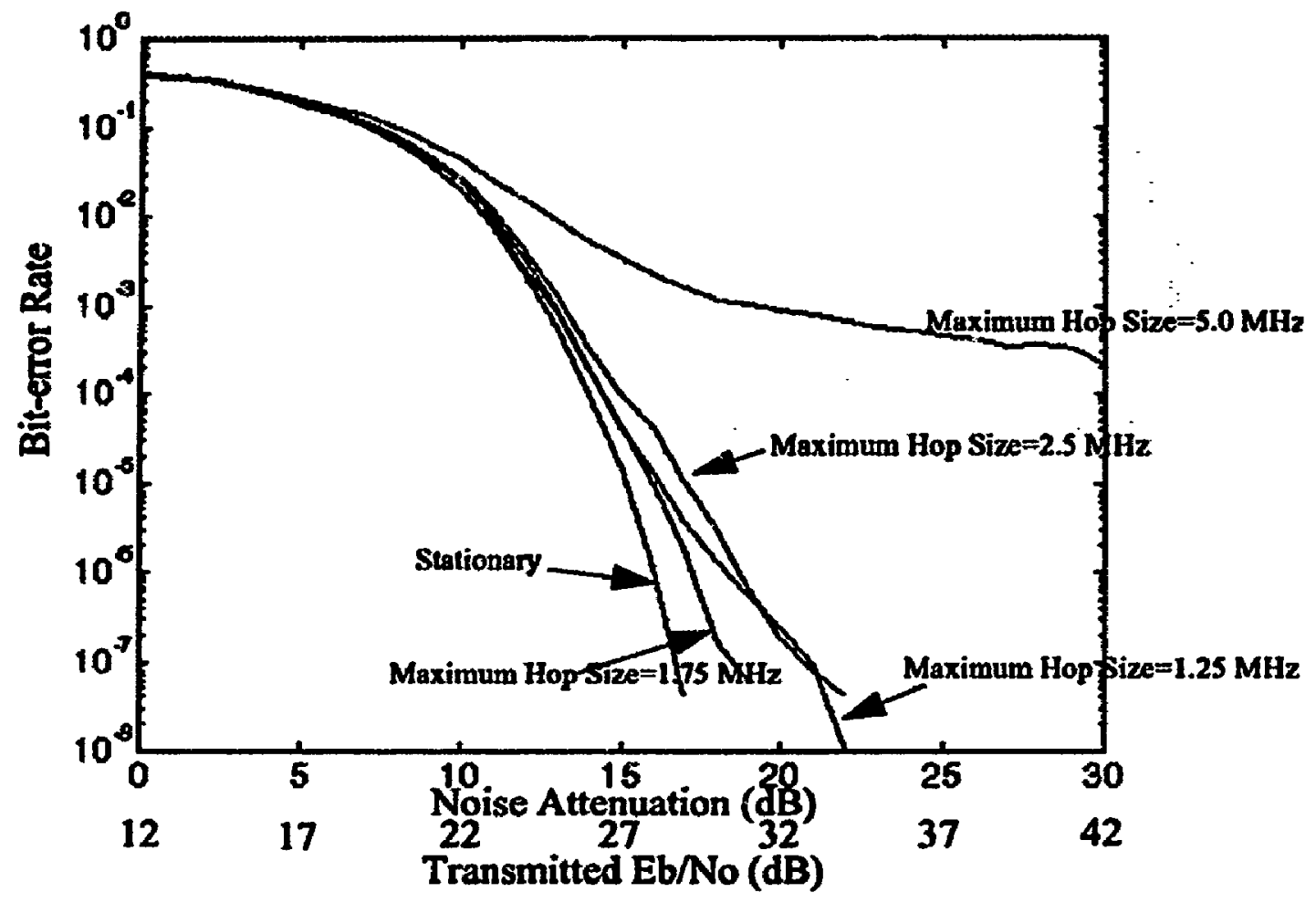

Figure 6-41: Bit-error Rate vs. Eb/No for Interpolated Frequency-hopping at $78 \mathrm{KHz}$

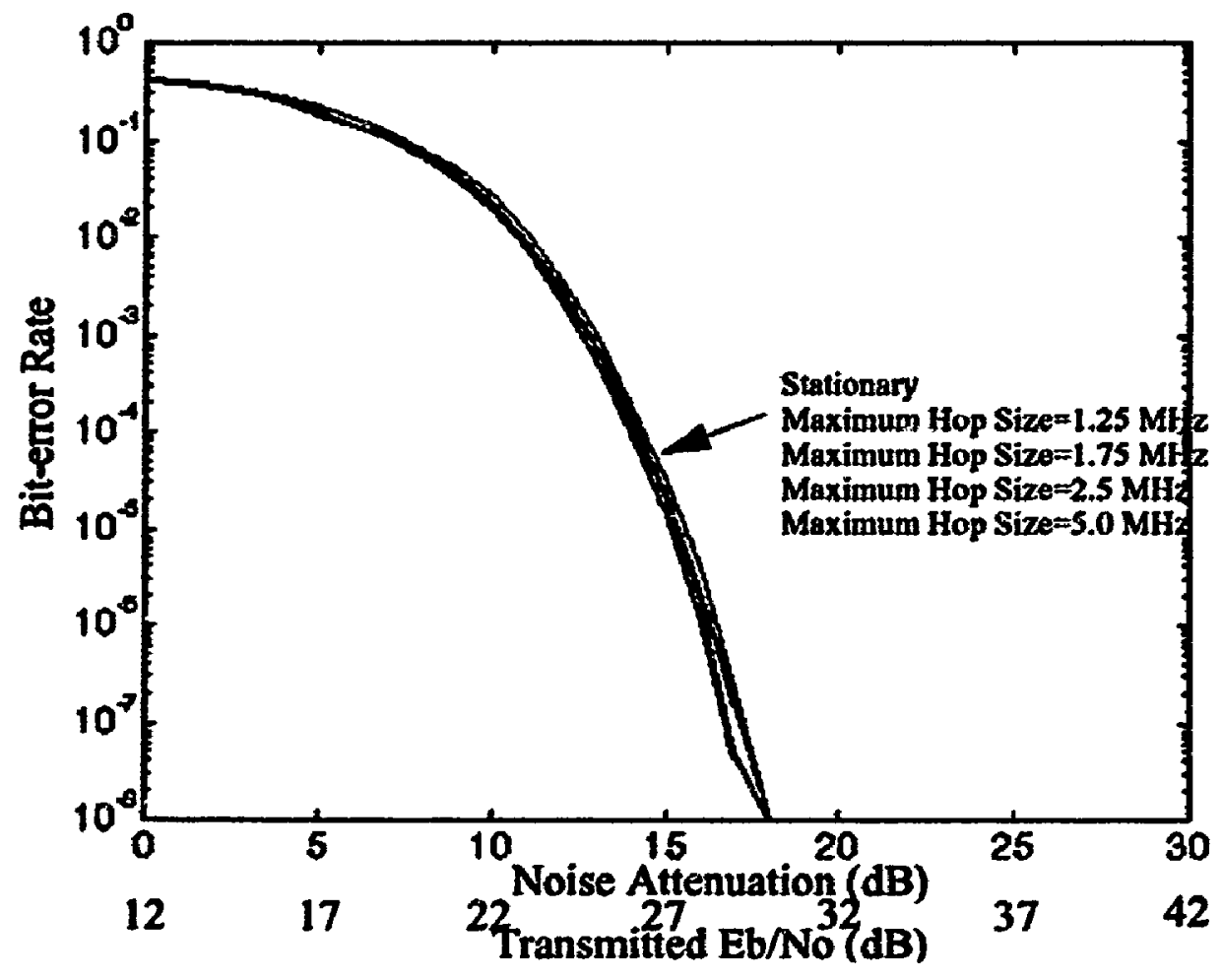


Figure 6-42: Bit Error Rate vs. Eb/No for Simple Frequency-hopping at $156 \mathrm{KHz}$

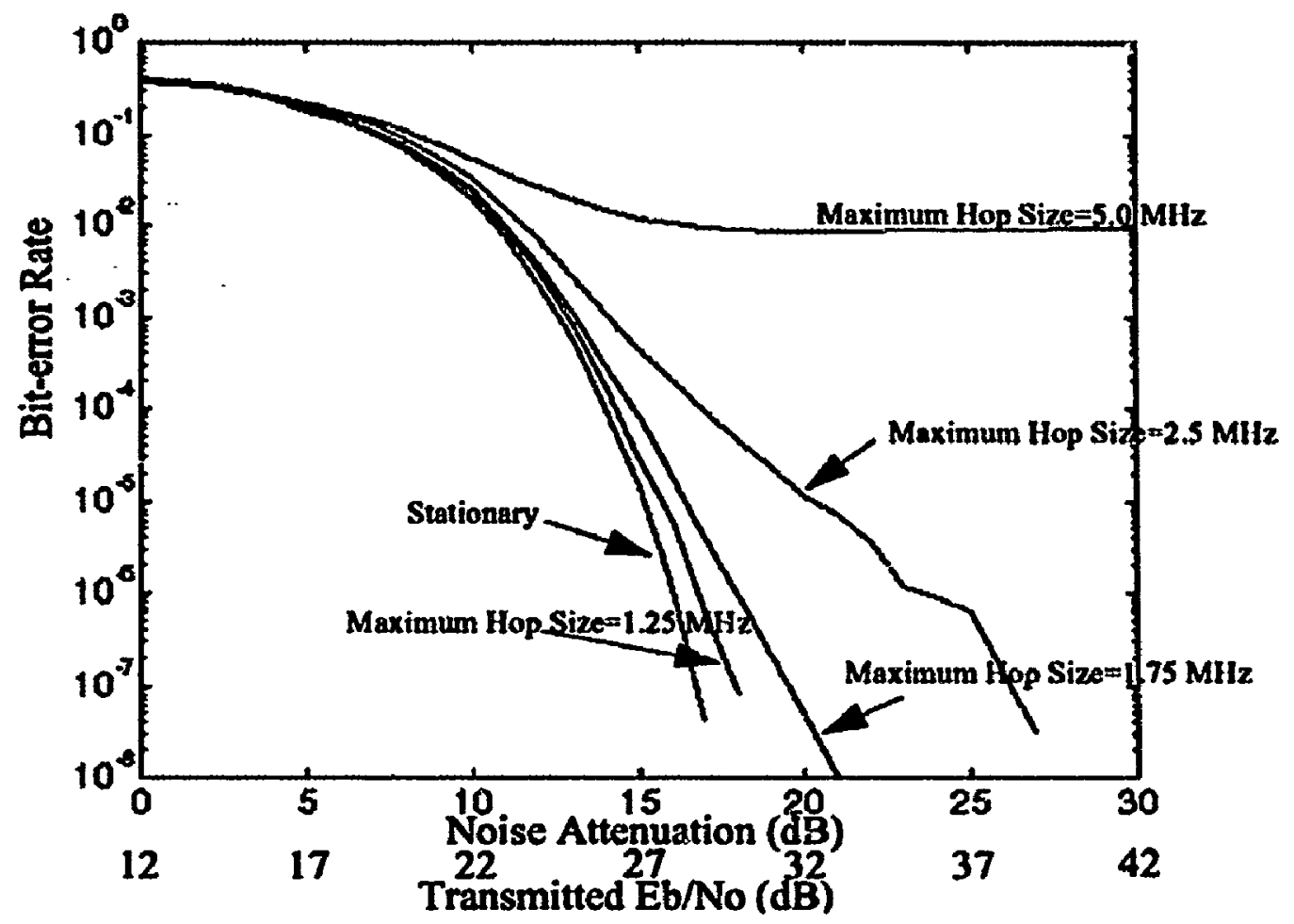

Figure 6-43: Bit-error Rate vs. Eb/No for Interpolated Frequency-hopping at 156KHz

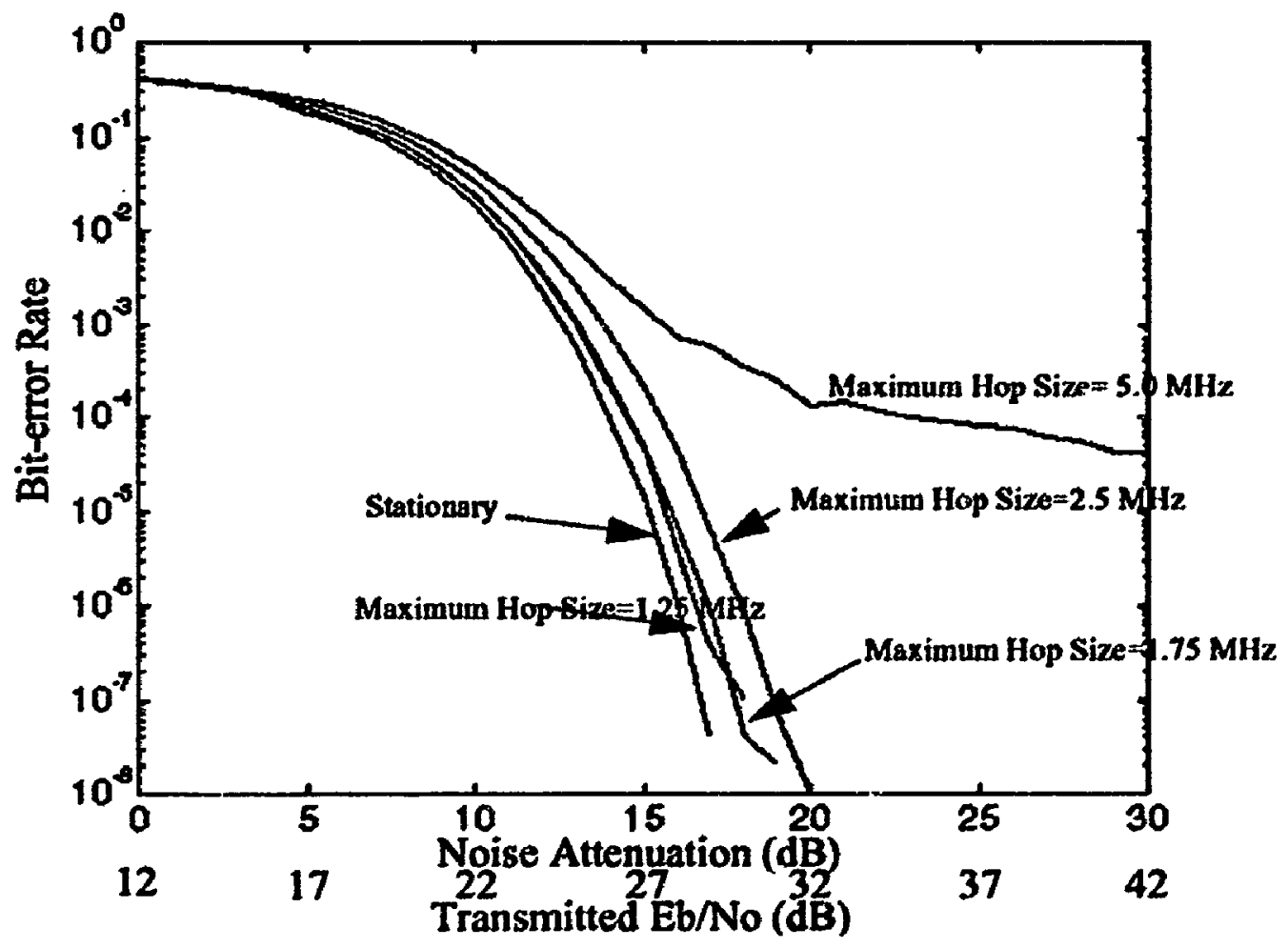


Figures (6-38) and (6-39) show the measured bit-error rate curves for simple frequencyhopping and interpolated frequency-hopping, for a code rate of $10 \mathrm{KHz}$. The bit-error rate curve for a stationary carrier is also included. It can be seen from these curves that for maximum hop sizes of $1.25 \mathrm{MHz}$ and $2.5 \mathrm{MHz}$, there is no noticeable difference between the stationary carrier case and both types of frequency-hopping. However, for a maximum hop size of $5.0 \mathrm{MHz}$, the minimum achievable bit-error rate for simple frequency-hopping transmission is approximately equal to $5 \times 10^{-3}$ regardless of the transmitted $\mathrm{Eb} / \mathrm{No}$. For interpolated frequency-hopping at a rate of $10 \mathrm{KHz}$, there is no noticeable difference between the stationary carrier performance and the interpolated frequency-hopping performance for a maximum hop size of $5.0 \mathrm{MHz}$.

Figures (6-40) and (6-41) show the measured bit-error rate curves for simple frequencyhopping and interpolated frequency-hopping, for a code rate of $78 \mathrm{KHz}$. The bit-error rate curve for a stationary carrier is also included. For simple frequency-hopping it can be seen from the curves that as the maximum hop sizes: are increased, the bit-error rate performance for high $\mathrm{Eb} / \mathrm{No}$ values worsens, and for a maximum hop size of $5.0 \mathrm{MHz}$ the minimum achievable bit-error rate is approximately equal to $9 \times 10^{-3}$, regardless of the transmitted $\mathrm{Eb} /$ No. For the interpolated frequency-hopping cases, a large performance improvement over the simple frequency-hopping cases can be seen from the curves, and there is no noticeable difference between interpolated frequency-hopping transmission and stationary carrier transmission for the maximum hop sizes tested.

Figures (6-42) and (6-43) show the measured bit-error rate virves for simple frequencyhopping and interpolated frequency-hopping, for a code rate of $156 \mathrm{KHz}$. The bit-error rate curve for a stationary carrier is also included. It can be seen from the curves for the simple frequency-hopping cases that the overall bit-error rate performance has degraded due to the increase in the hopping rate, and becomes worse as larger maximum hop sizes are used. For a maximum hop size of $5.0 \mathrm{MHz}$, the minimum achievable bit-error rate for standard frequency-hopping is $1 \times 10^{-2}$, regardless of the transmitted $\mathrm{Eb} / \mathrm{No}$. For the interpolated frequercy-hopping case, the increased hopping rate has also degraded the bit-error rate performance of the system, and as the maximum hop sizes increase, a further degradation 
in performance is seen. For a maximum hop size of $5.0 \mathrm{MHz}$, the minimum achievable biterror rate for interpolated frequency-hopping transmission is $5 \times 10^{-4}$, regardless of the transmitted $\mathrm{Eb} / \mathrm{No}$. However, the performance obtained using interpolated frequencyhopping is still better than that achieved with simple frequency-hopping.

Table (6-2) shows the required transmitted $\mathrm{Eb} / \mathrm{No}$ values needed to achieve bit error rates of $10^{-3}, 10^{-4}$, and $10^{-5}$ using simple frequency-hopping and interpolated frequencyhopping transmission for various hopping rates and maximum hop sizes. Note that for mobile radio applications, bit-error rates of $10^{-3}$ or $10^{-4}$ are probably acceptable, but proulems such as spectral-splatter still need to be addressed. 
Table 6-2: Bit-error Rate for Given Eb/No Values

\begin{tabular}{|l|l|l|l|}
\hline Hopping Rate and Maximum Hop Size & $\begin{array}{c}\text { BER=10-3 } \\
\text { Eb/No (dB) }\end{array}$ & $\begin{array}{l}\text { BER=10-4 } \\
\text { Eb/No(dB) }\end{array}$ & $\begin{array}{l}\text { BER=10-5 } \\
\text { Eb/No(dB) }\end{array}$ \\
\hline \hline Stationary Carrier & 25.1 & 26.4 & 27.7 \\
\hline Hop rate=10 KHz, Hop size=1.25 MHz & $\begin{array}{l}\text { FH=25.2 } \\
\text { IFH=25.2 }\end{array}$ & $\begin{array}{l}\text { FH=26.5 } \\
\text { IFH=26.5 }\end{array}$ & $\begin{array}{l}\text { FH=27.7 } \\
\text { IFH=27.7 }\end{array}$ \\
\hline Hop rate=10 KHz, Hop size=2.5 MHz & $\begin{array}{l}\text { FH=25.1 } \\
\text { IFH=25.2 }\end{array}$ & $\begin{array}{l}\text { FH=26.4 } \\
\text { IFH=26.5 }\end{array}$ & $\begin{array}{l}\text { FH=27.9 } \\
\text { IFH=27.8 }\end{array}$ \\
\hline Hop rate=10 KHz, Hop Size=5.0 MHz & $\begin{array}{l}\text { FH=26.2 } \\
\text { IFH=25.3 }\end{array}$ & $\begin{array}{l}\text { FH=N/A } \\
\text { IFH=26.7 }\end{array}$ & $\begin{array}{l}\text { FH=N/A } \\
\text { IFH=28.0 }\end{array}$ \\
\hline Hop rate=78 KHz, Hop size=1.25 MHz & $\begin{array}{l}\text { FH=25.3 } \\
\text { IFH=25.4 }\end{array}$ & $\begin{array}{l}\text { FH=27.0 } \\
\text { IFH=26.8 }\end{array}$ & $\begin{array}{l}\text { FH=28.4 } \\
\text { IFH=28.0 }\end{array}$ \\
\hline Hop rate=78 KHz, Hop size=1.75 MHz & $\begin{array}{l}\text { FH=25.3 } \\
\text { IFH=25.3 }\end{array}$ & $\begin{array}{l}\text { FH=27.0 } \\
\text { IFH=26.7 }\end{array}$ & $\begin{array}{l}\text { FH=28.4 } \\
\text { IFH=27.9 }\end{array}$ \\
\hline Hop rate=78 KHz, Hop size=2.5 MHz & $\begin{array}{l}\text { FH=25.7 } \\
\text { IFH=25.3 }\end{array}$ & $\begin{array}{l}\text { FH=27.4 } \\
\text { IFH=26.6 }\end{array}$ & $\begin{array}{l}\text { FH=29.4 } \\
\text { IFH=28.0 }\end{array}$ \\
\hline Hop rate=78 KHz, Hop size=5.0 MHz & $\begin{array}{l}\text { FH=31.6 } \\
\text { IFH=25.4 }\end{array}$ & $\begin{array}{l}\text { FH=N/A } \\
\text { IFH=27.0 }\end{array}$ & $\begin{array}{l}\text { FH=N/A } \\
\text { IFH=28.7 }\end{array}$ \\
\hline Hop rate=156 KHz, Hop size=1.25 MHz & $\begin{array}{l}\text { FH=25.2 } \\
\text { IFH=25.3 }\end{array}$ & $\begin{array}{l}\text { FH=26.6 } \\
\text { IFH=26.9 }\end{array}$ & $\begin{array}{l}\text { FH=28.0 } \\
\text { IFH=28.1 }\end{array}$ \\
\hline Hop rate=156 KHz, Hop size=1.75 MHz & $\begin{array}{l}\text { FH=25.5 } \\
\text { IFH=25.5 }\end{array}$ & $\begin{array}{l}\text { FH=27.3 } \\
\text { IFH=27.2 }\end{array}$ & $\begin{array}{l}\text { FH=29.0 } \\
\text { IFH=28.3 }\end{array}$ \\
\hline Hop rate=156 KHz, Hop size=2.5 MHz & $\begin{array}{l}\text { FH=26.5 } \\
\text { IFH=26.3 }\end{array}$ & $\begin{array}{l}\text { FH=29.4 } \\
\text { IFH=28.1 }\end{array}$ & $\begin{array}{l}\text { FH=32.7 } \\
\text { IFH=29.3 }\end{array}$ \\
\hline Hop rate=156 KHz, Hop Size=5.0 MHz & $\begin{array}{l}\text { FH=N/A } \\
\text { IFH=28.0 }\end{array}$ & $\begin{array}{l}\text { FH=N/A } \\
\text { IFH=35.3 }\end{array}$ & $\begin{array}{l}\text { FH=N/A } \\
\text { IFH=50.5 }\end{array}$ \\
\hline
\end{tabular}

It can be seen from the results summarized in Table (6-2) that for lower frequency deviations and hopping-rates, there is very little difference in the transmitted $\mathrm{Eb} / \mathrm{No}$ required to achieve a certain bit-error rate for either simple frequency-hopping or interpolated frequency-hopping. However, as the maximum hop sizes and hopping rates become larger, it is possible to achieve the same bit-error rate using a smaller transmitted 
$\mathrm{Eb} / \mathrm{No}$ for interpolated frequency-hopping than is possible using simple frequencyhopping. There are also some cases where it is possible to transmit at a given bit-error rate with interpolated frequency-hopping where it would not be possible to use simple frequency-hopping.

Figire (6-44) shows a plot of the bit-error rate curve obtained for stationary carrier transmission measured using the system tested in this thesis, along with a plot of the theoretical bit-error rate curve for non-coherent FSK transmission.

Figure 6-44: Bit-error Rate vs. Eb/No for both the Stationary Carrier Case and The Ideal Non-coherent FSK Case

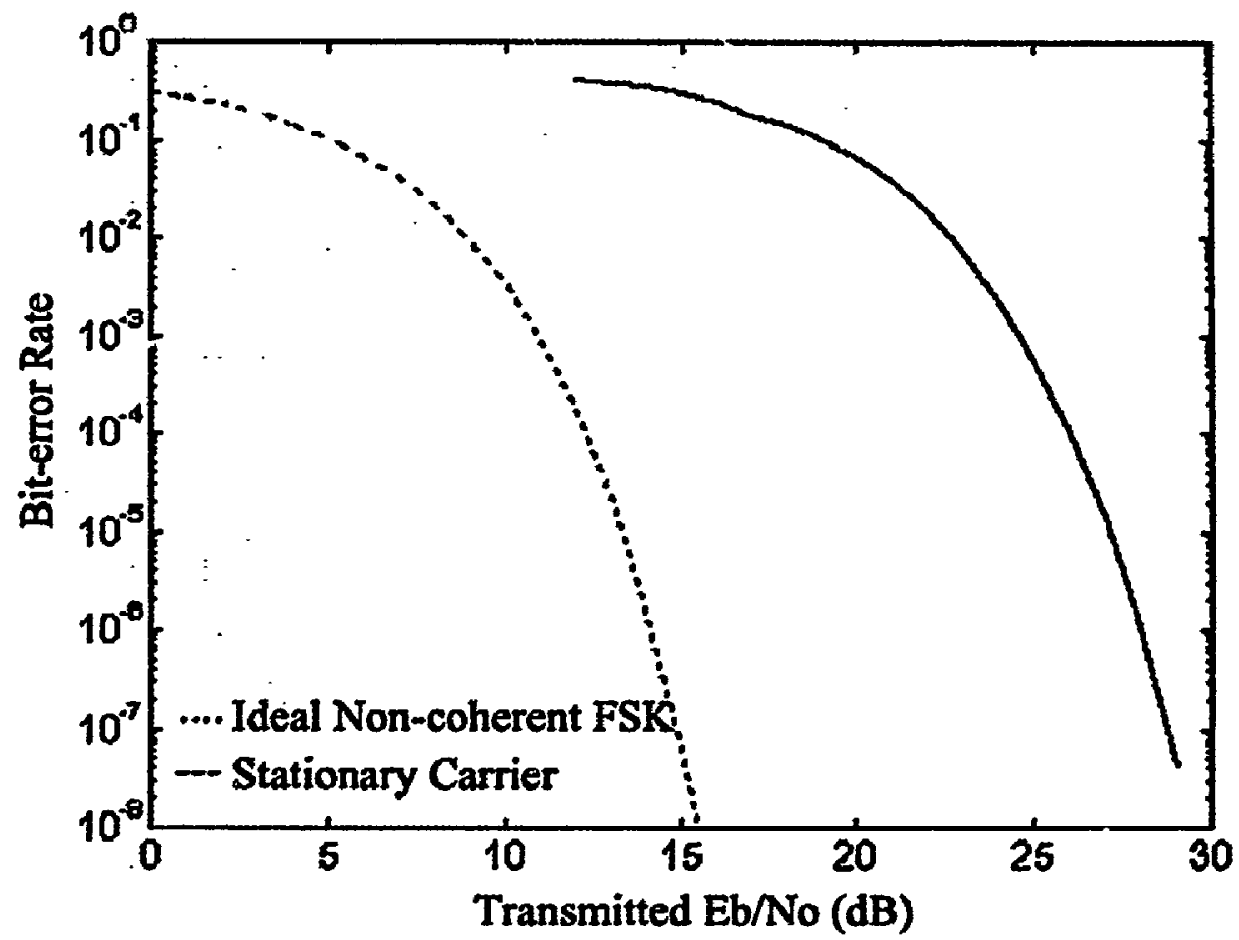

Although the two bit-error rate curves shown in Figure (6-44) have the same shape, there is approximately a $12 \mathrm{~dB}$ offset between the $\mathrm{Eb} / \mathrm{No}$ values required to achieve a given biterror rate in the stationary case when compared to the ideal non-coherent case. The cause of this offset can be explained. The theoretical bit-error rate curve for the noncoherent case is calculated assuming that the minimum bandwidth possible for the FSK data deviations 
is employed, and that the added channel noise is limited to this bandwidth as well. For the stationary carrier case tested in this thesis, data deviations of $+1-500 \mathrm{KHz}$ were employed for a 78 $\mathrm{Kb} / \mathrm{s}$ data rate. Assuming that the added noise is perfectly limited to a $1 \mathrm{MHz}$ bandwidth around the carrier, the measured transmitted SNR for a transmitted Eb/No value of $12 \mathrm{~dB}$ is approximately $1 \mathrm{~dB}$. This low transmitted SNR is the source of the offset between the two bit-error rate curves. If the data were transmitted using the minimum frequency deviations possible, the measured SNR for a transmitted Eb/No of $12 \mathrm{~dB}$ would be approximately $12 \mathrm{~dB}$. The ratio of the bandwidth employed and the ideal bandwidth is approximately $11 \mathrm{~dB}$. Other sources of offset between the two curves, are phase-noise in the transmit and receive frequency synthesizers, non-ideal filtering, nonidealities in the FSK demodulator, and the fact that the matched filter in the FSK demodulator is matched to a boxcar shaped pulse rather than the step response of the PLL. 


\section{Chapter 7}

\section{Conclusions and Future Work}

\subsection{Conclusions}

In order to meet the increasing demands on system designers imposed by mobile radio environments, the use of spread-spectrum transmission methods for commercial applications is being considered. One of the common forms of spread-spectrum, PLL based frequency-hopping, performs well in such environments, but suffers from problems such as spectral-splatter and IF frequency-error produced by PLL synthesizer mismatch, which reduce system efficiency and performance. These problems are usually controlled using VCO pre-tuning, multiple synthesizers, or transient guard and dwell times. In this thesis, interpolated frequency-hopping has been presented as another solution to these problems, reducing spectral-splatter and IF error and reducing the bit-error rate, as well as providing the receiver with a continuous stream of data that can be sampled over the entire symbol interval using oversampling techniques such as the $\Delta-\Sigma$ frequency discriminator discussed in Chapter 4. This provides an opportunity for improvements in the received IF power.

Interpolation of the hopping code is used to reduce the high frequency content of the hopping steps, thereby reducing the PLL transient phase error. The result of this filtering is a carrier that moves smoothly and continuously in frequency. Laboratory tests show that interpolated frequency-hopping reduces spectral-splatter and phase-noise in the receiver IF when compared to simple frequency hopping, especially for rates approaching or exceeding the PLL corner frequency. It has been shown that higher hopping rates can be used for interpolated frequency-hopping than could be used with simple frequencyhopping for a given synthesizer, while maintaining the same bit-error rate in the receiver. This is because interpolated frequency-hopping generates constant phase error in the PLL which increases when the code rate increases. As the code rate increases, the SNR of the receiver decreases, and eventually, reception becomes impossible. In simple frequencyhopping, as code rates begin to approach the loop settling time, dwell times become unusable and reception also becomes impossible. This transition is more gradual with 
interpolated frequency-hopping than with simple frequency-hopping. The results of the biterror rate tests performed in the laboratory show that the bit-error rates achievable through the use of interpolated frequency-hopping transmission would be suitable for mobile radio applications, and would allow designers to take fuller advantage of the system used.

The laboratory results obtained also show that fractional- $\mathrm{N}$ synthesis using $\Delta-\Sigma$ modulated control bitstreams provides the spread-spectrum system with the high spectral quality, high frequency resolution, and agility required to accurately control the transmitter output signal digitally.

By shaping the frequency-hopping through the use of a digital interpolation filter we have increased the digital complexity of the system, but have reduced the analog problems. As VLSI technologies continue to progress, this kind of trade off becomes increasingly favorable to the approach presented here, particularly for applications requiring low power consumption and small size.

\subsection{Future Work}

Although the introduction of this thesis describes multi-path fading and diversity, no experimental results were obtained in this area due to equipment and time constraints. However, the section is included because the ultimate purpose for using spread-spectrum transmission in commercial applications is to obtain some degree of immunity to multipath effects. Future work would include the testing of interpolated frequency-hopping transmission in a multi-path environment. The issue of code phase synchronization between the transmitter and receiver would be investigated, as well as the possibility of using PDMA in a multi-user environment. A full mathematical analysis of the properties of interpolated frequency-hopping would also be required.

Work is currently underway to implement hardware versions of the hopping code generator and interpolation filter, as well as the digital $\Delta-\Sigma$ modulator used to control the frequency synthesizers. These circuits will allow easier testing of the interpolated frequency-hopping 
radio system, and move the design one step closer to a full on chip implementation. 


\section{References}

[AG89] J. R. Alexovich and R. M. Gagliardi, "Effect of PLL Synthesizer in FSK Frequency-Hopped Communications," IEEE Trans. Commun., Vol.37, No. 3, pp. 268-275, March 1989.

[AS83] Tor Aulin and Carl-Erik Sundberg, "An Easy Way to Calculate Power Spectra for Digital FM," IEE Proceedings, Vol. 130, Part F, No.6, pp. 519-526, October 1983.

[Bax93] Walt Bax, "Frequency Synthesis for Telecommunications," internal document, Carleton University, 1992.

[BC93] R. D. Beards and M. A. Copeland, "An Oversampled Delta-Sigma Discriminator for Digital Radio:" 1993 CCVLSI, Banff, Alberta, November 1993.

[BC94] R. D. Beards and M. A. Copeland, "An Oversampling Delta-Sigma Frequency Discriminator," IEEE Transactions on Circuits and Systems, Vol. 41, No. 1, pp. 26-32, January 1994.

[BF86] J. S. Bird and E. B. Felstead, “Antijam Performance of Fast Frequency-hopped M-ary NCFSK-An Overview," IEEE Journal on Selected Areas in Communications, Vol. SAC-4, No.2, pp. 216-232, March 1986.

[Bla92] Alain Blanchard, Phase-Locked Loops: Application to Coherent Receiver Design, Malabar, Florida: Krieger Publishing Company., 1192.

[Craw94] James A. Crawford, Frequency Synthesizer Design Handbook, Norwood, MA: Artech House, Inc., 1994. 
[CT91] J. C. Candy and G. C. Temes, "Oversampling Methods for AVD and D/A Conversion," Oversampling Dilta-Sigma Converters, New York: IEEE Press, 1991.

[Don92] Adam Dong, "Incorporation of Digital Angle-Modulation into a Fractional Division Synthesizer," Masters Thesis, Carleton University, Ottawa, Ontario, April 1992.

[Fis91] Stewart Fist, “'Spread Spectrum' Cellular Phones," Electronics Australia, pp. 22-26, March 1991.

[Gib93] Jerry D. Gibson, Digital and Analog Communications, USA: Macmillan Publishing Company., 1993.

[Ken94] T. P. Kenny, "Components and Architectures for an Integrated Wideband FM Transceiver," Masters Thesis, Carleton University, Ottawa, Ontario, 1994.

[Ker92] Richard Kerr, "The CDMA Digital Cellular System An ASIC Overview," IEEE 1992.

[KM87] M. Kavehrad and P.J. McLane, "Spread Spectrum for Indonr Digital Radio," IEEE Communications Magazine, Vol. 25, No. 6, pp. 32-40, June 1987.

[KS75] Peter Kabal and Subbarayan Pasupathy, "Partial-Response Signalling," IEEE Trans. Commun., Vol. COM-23, No.9, pp. 921-934, September 1975.

[Lef94? Barry J. Leff, "Making Sense of Wireless Standards and System Designs," Microwaves \& RF, pp. 113-118, February 1994. 
[LW90] Y. M. Lam and P. H. Wittke, "Frequency-Hopped Spread-Spectrum Transmission With Band-Efficient Modulations and Simplified Noncoherent Sequence Estimation," IEEE Trans. Commun., Vol. 38, No. 12 pp. 2184-2195, December 1990.

[MAT92] The Math Works Inc., MATLAB Reference Guide, Natick Mass., 1992.

[MC90] Brian Miller and Bob Conley, "A Multiple Modulator Fractional Divider," IEEE Symposium on Frequency Control, pp. 559-567, May 1990.

[Mic91] News Brief, "ICU accepts Qualcomm's CDMA," Microwaves \& RF, pg. 25, May 1994.

[MLT91] L. E. Miller, J.S. Lee and D. J. Torrieri, "Analysis of Transients Affecting The Selection of Hopping Rate For FH/CPFSK Systems," IEEE, Milcom '91, pp. 0590-0600.

[NTR94] Northem Telecom Ltd. and National Research Council (NRC), Ottawa Citizen, May 20, 1994.

[Pro83] John G. Proakis, Digital Communications, USA: McGraw Hill Inc., 1983.

[PSM82] R. L. Pickholtz, D. L. Schilling and L. B. Milstein, "Theory of Spread-Spectrum Communications - A Tutorial," IEEE Trans. Commun., Vol. COM-30, pp. 855-884, May 1982.

[RC94] T. A. D. Riley and M. A. Copeland, "A Simplified Continuous Phase Modulator Technique," IEEE Trans, on Circuits \& Systems, Vol.41, No.5, May 1994. 
[RCK93] T. A. Riley, M. A. Copeland and T. A. Kwasniewski, "Delta-sigma Modulation in Fractional-N Frequency Synthesis," IEEE Joumal of Solid-State Circuits, Vol.28, No.5, pp. 553-559, May 1993.

[RD77] M. P. Ristenbatt and J. L. Daws, Jr., "Performance Criteria for Spread-Spectrum Communications," IEEE Trans. Commun., Vol. COM-25, pp. 756-762, August 1977.

[Ril91] T. A. Riley, Oversampled Analog to Digital Conversion Evaluation Software ROSAD, 2nd ed., Carleton University, Dept. of Electronics, 1991.

[Rod91] Martin S. Roden, Analog and Digital Communication Systems, Englewood Cliffs, New Jersey: Prentice Hall., 1991.

[RW90] D. N. Romalo and Q. Wang, "Design and Implementation of A FrequencyHopping Modem," Canadian Conference on Electrical and Computer Engineering, Ottawa, Ontario, Canada, September 1990.

[Sch82] R. A. Scholtz, "The Origins of Spread-Spectrum Communications," IEEE Trans. Commun., Vol. COM-30, pp. 822-854, May 1982.

[SPI] L. G. Meares and C. E. Hymowitz, Simulating with SPICE, Intusoft, 1988.

[Stre79] Ferrel G. Stremler, Introduction to Communication Systems, Addison-Wesley Publishing Company., 1979.

[Sun86] Carl-Erik Sundberg, "Continuous Phase Modulation," IEEE Cornmunications Magazine, Vol. 24, No.4, pp. 25-38, April 1986. 
References

120

[SV87] Adel A. M. Sale and Reinaldo A. Valenzuela, "A Statistical Model for Indoor Multipath Propagation," IEEE Journal on Selected Areas in Communecations, Vol. SAC-5, No.2, pp. 128-137, February 1987.

[TA85] Y. Tsividis and P. Antognetti, Editors, Design of MOS VLSI Circuits for Pelecommunications, USA: Prentice-Hall, Inc., 1985.

[Tor85] D. J. Torrieri, Principles of Secure Communication Systems, Dedham MA: Artech House, 1985

[VMS84] Didier Verhulst, Michel Mouly and Jacques Szpirglas, "Slow Frequency Hopping Multiple Access for Digital Cellular Radiotelephone," IEEE Journal on Selected Areas in Communications, Vol. SAC-2, No.4, pp. 563-574, July 1984. 


\section{Appendix A}

\section{BER Board Schematics}

This section contains the schematic diagrams for the real-time bit-error rate board.

A.1 


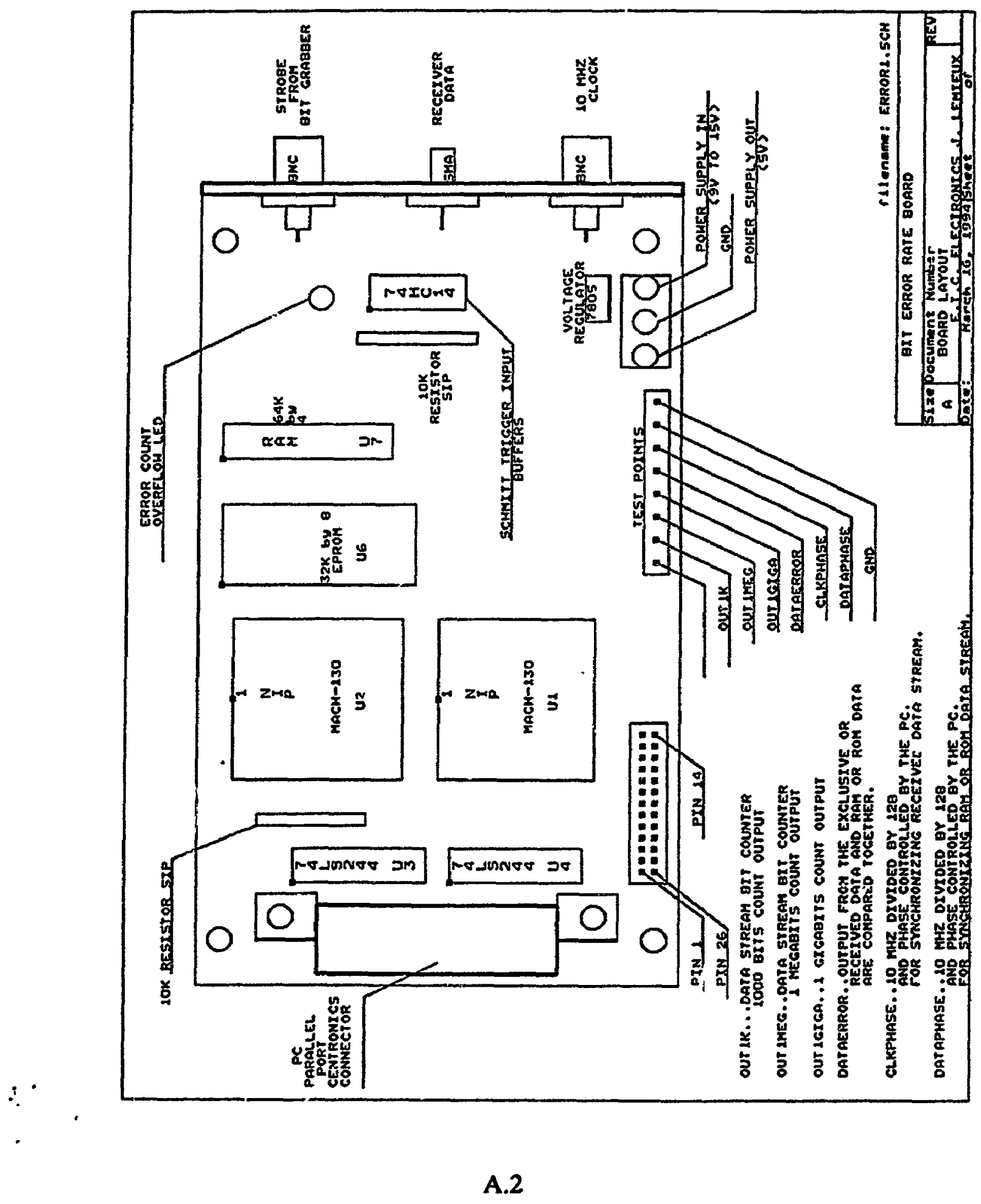




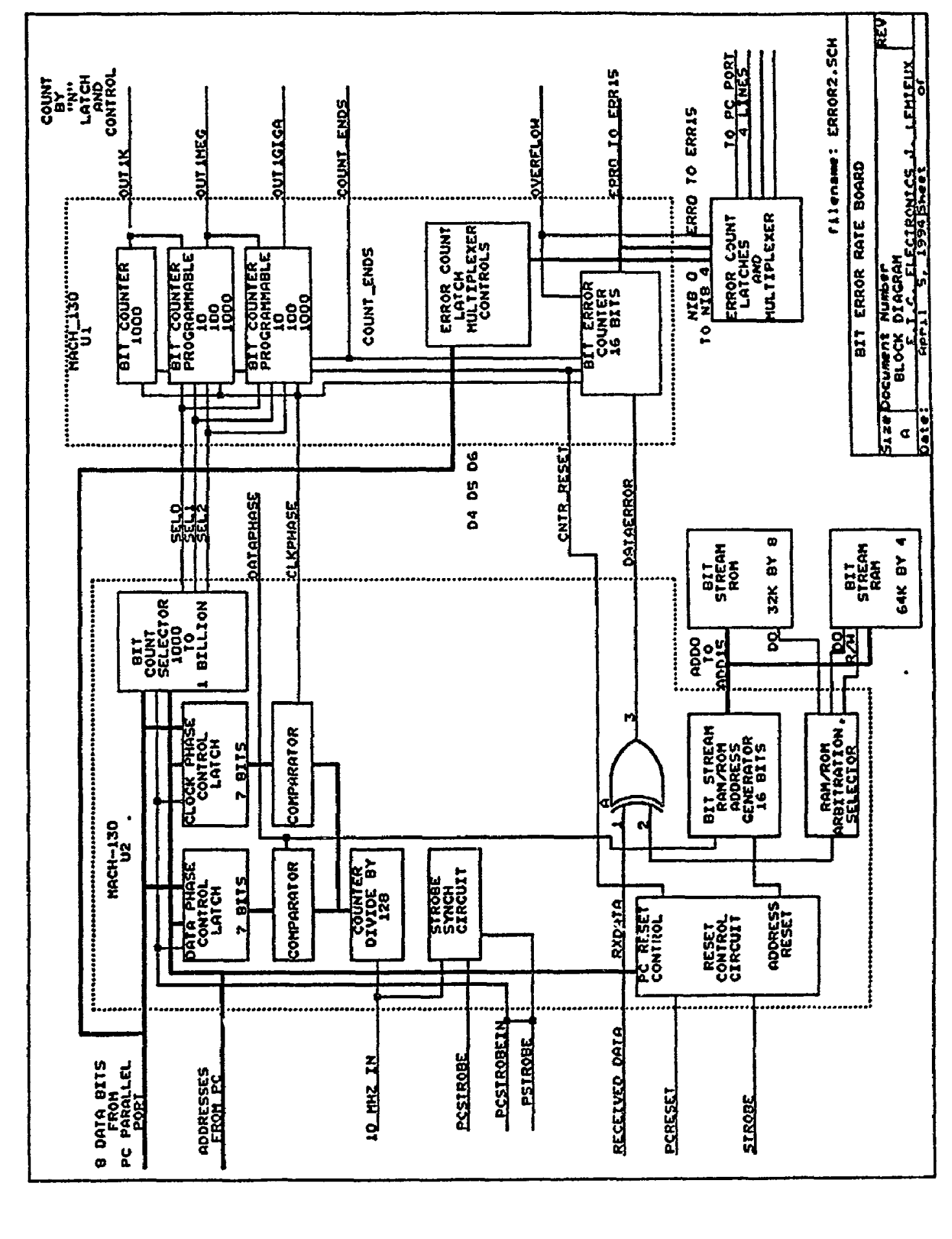

A. 3 


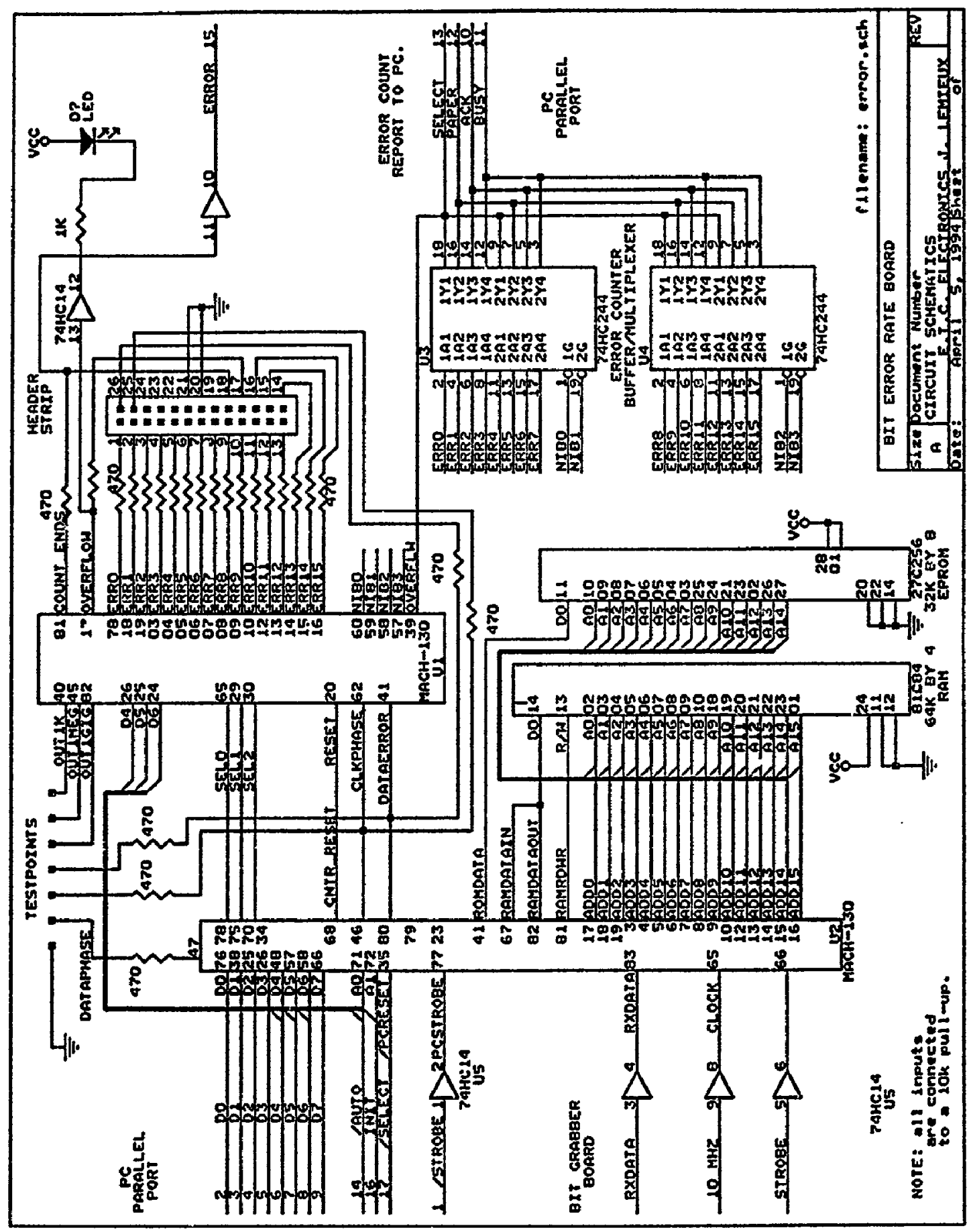

A. 4 


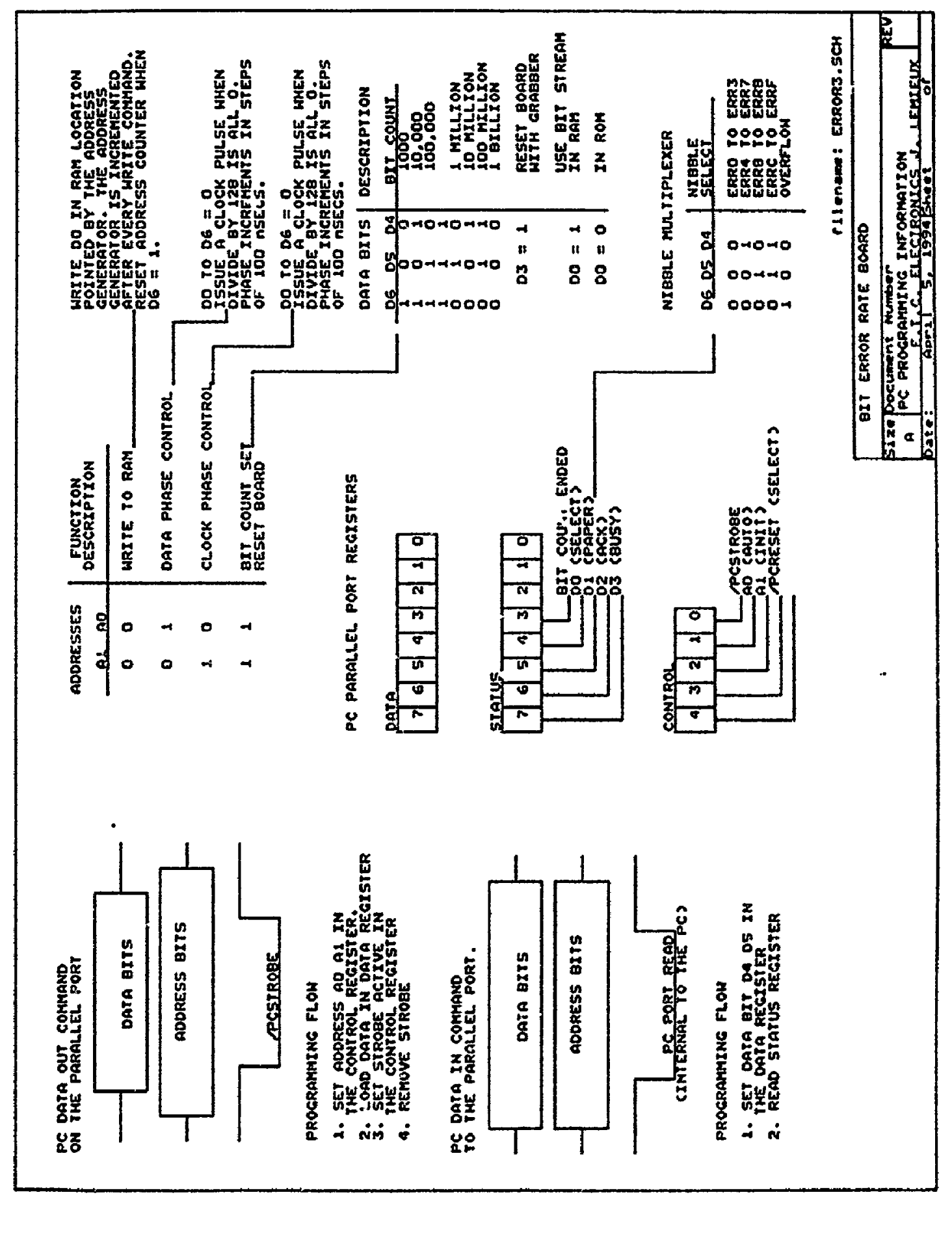




\section{Appendix B}

\section{FSK Demodulator Board Circuit Schematics}

This section contains circuit diagrams for the FSK demodulator board. 


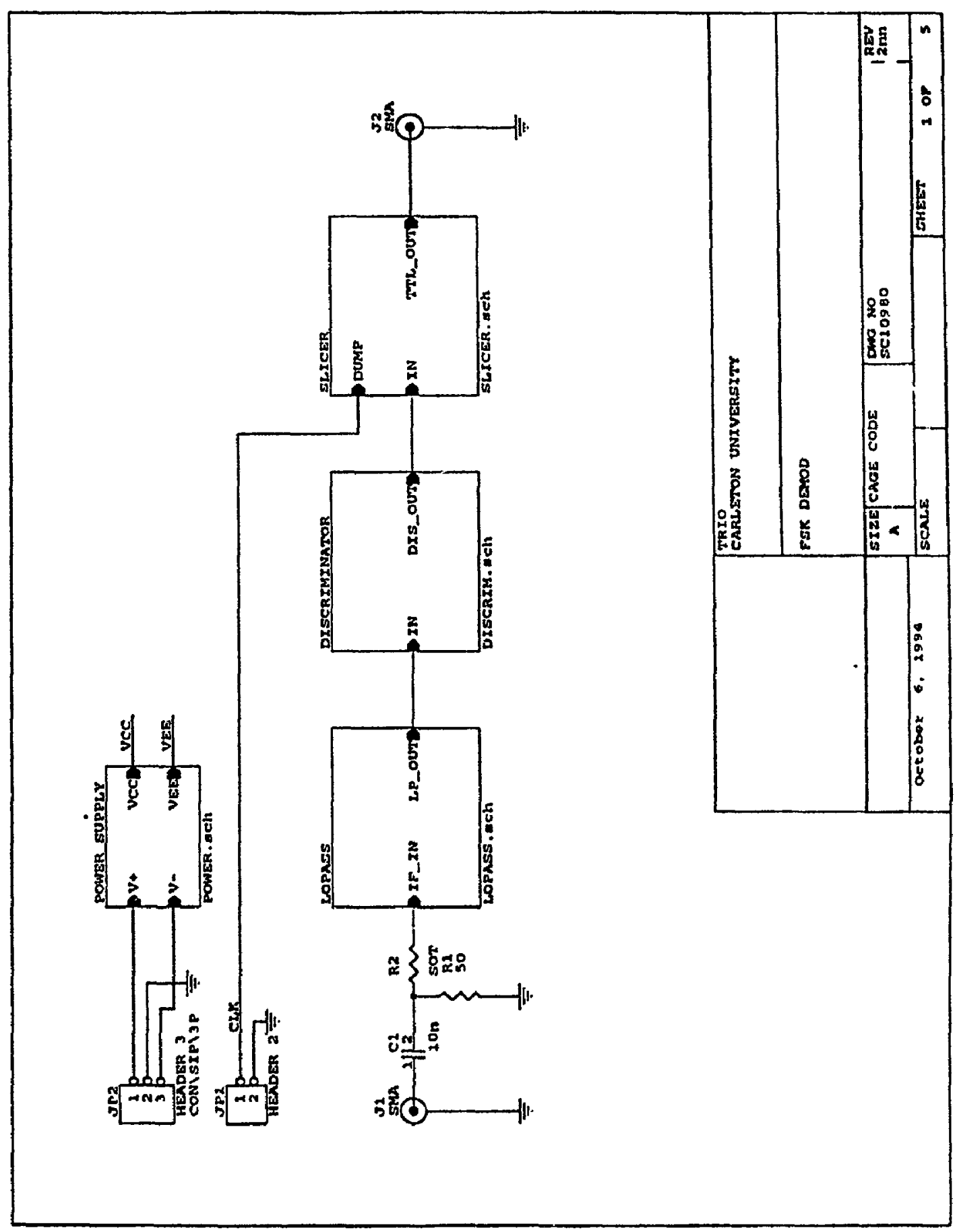

B. 2 


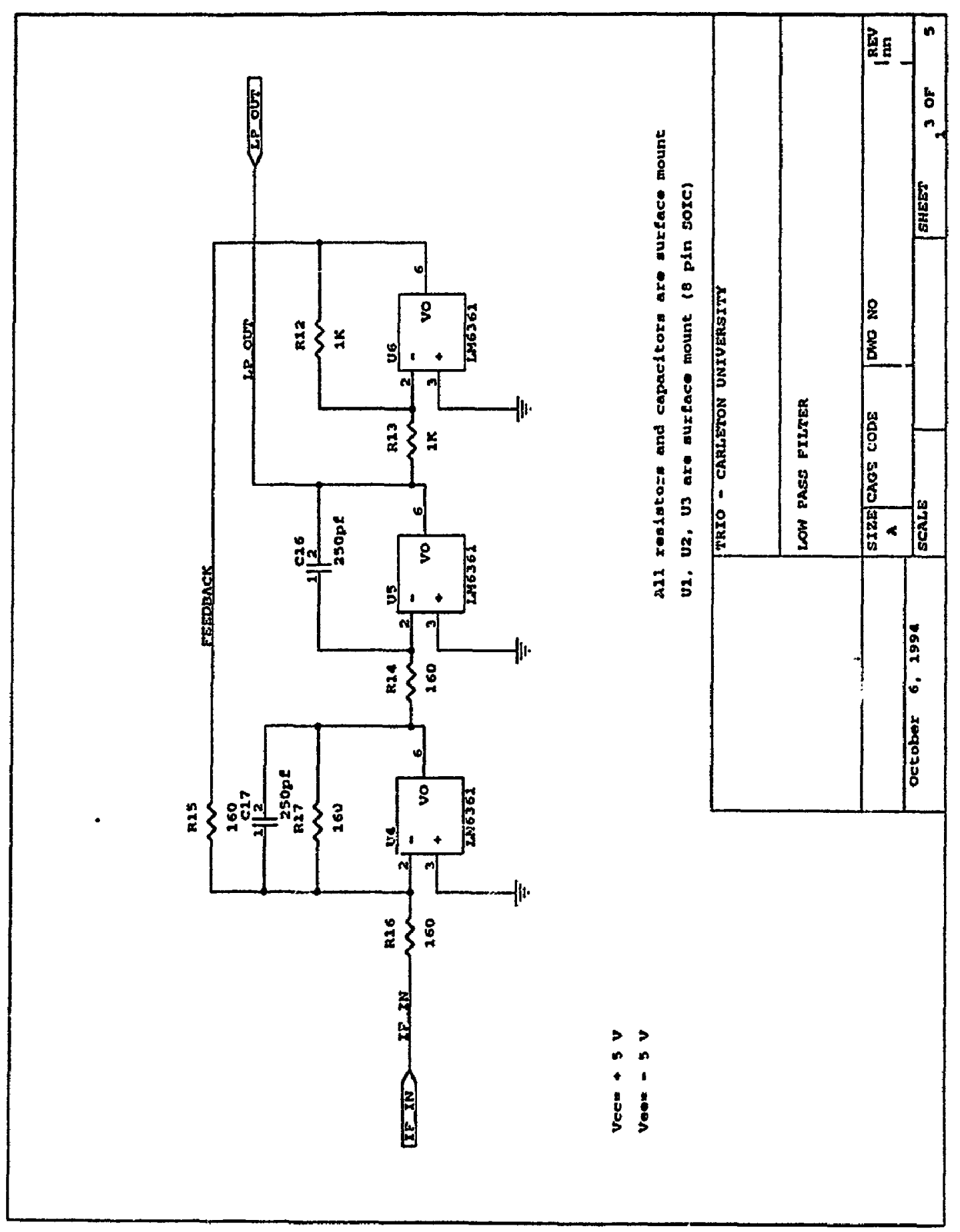

B.3 


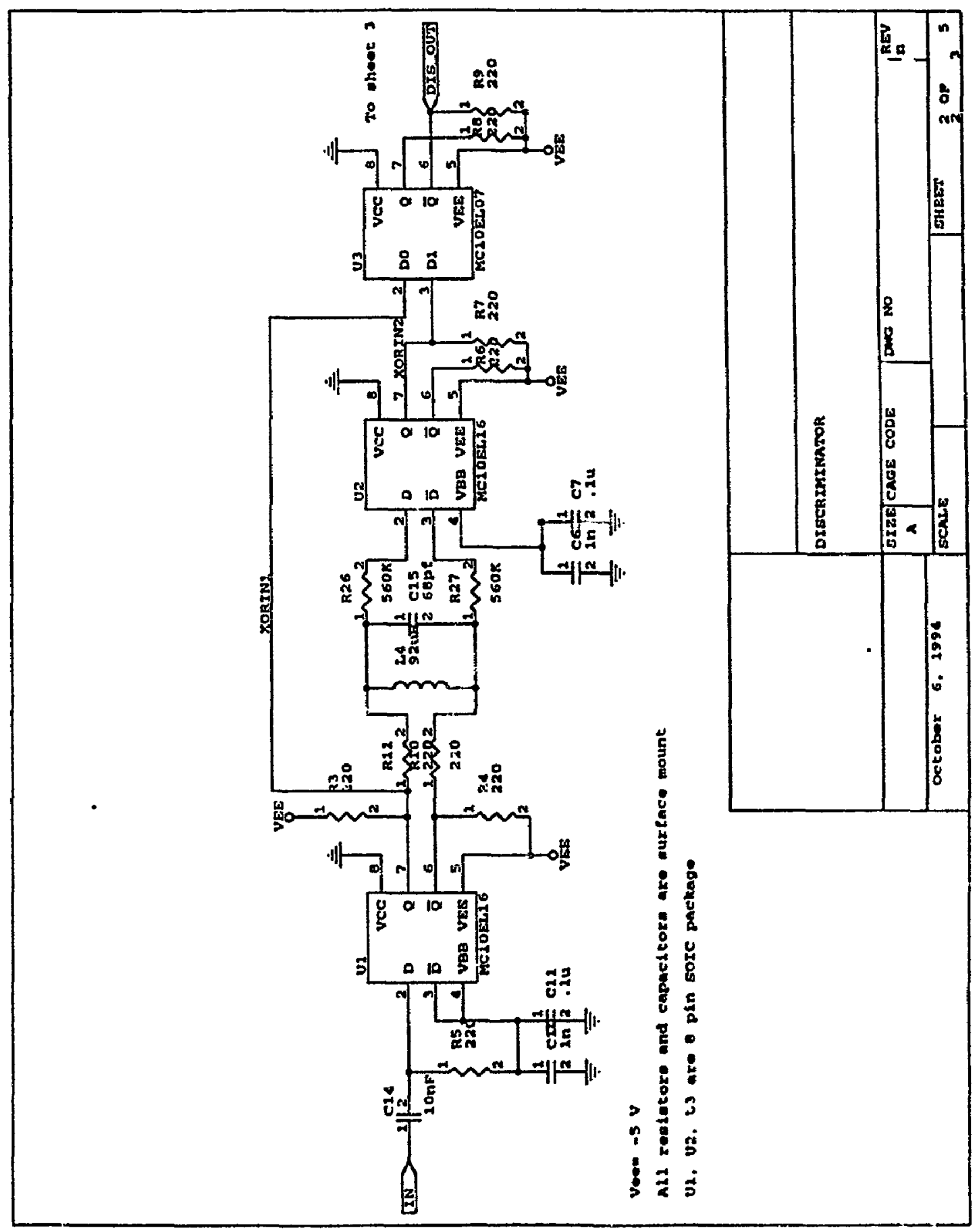

B.4 


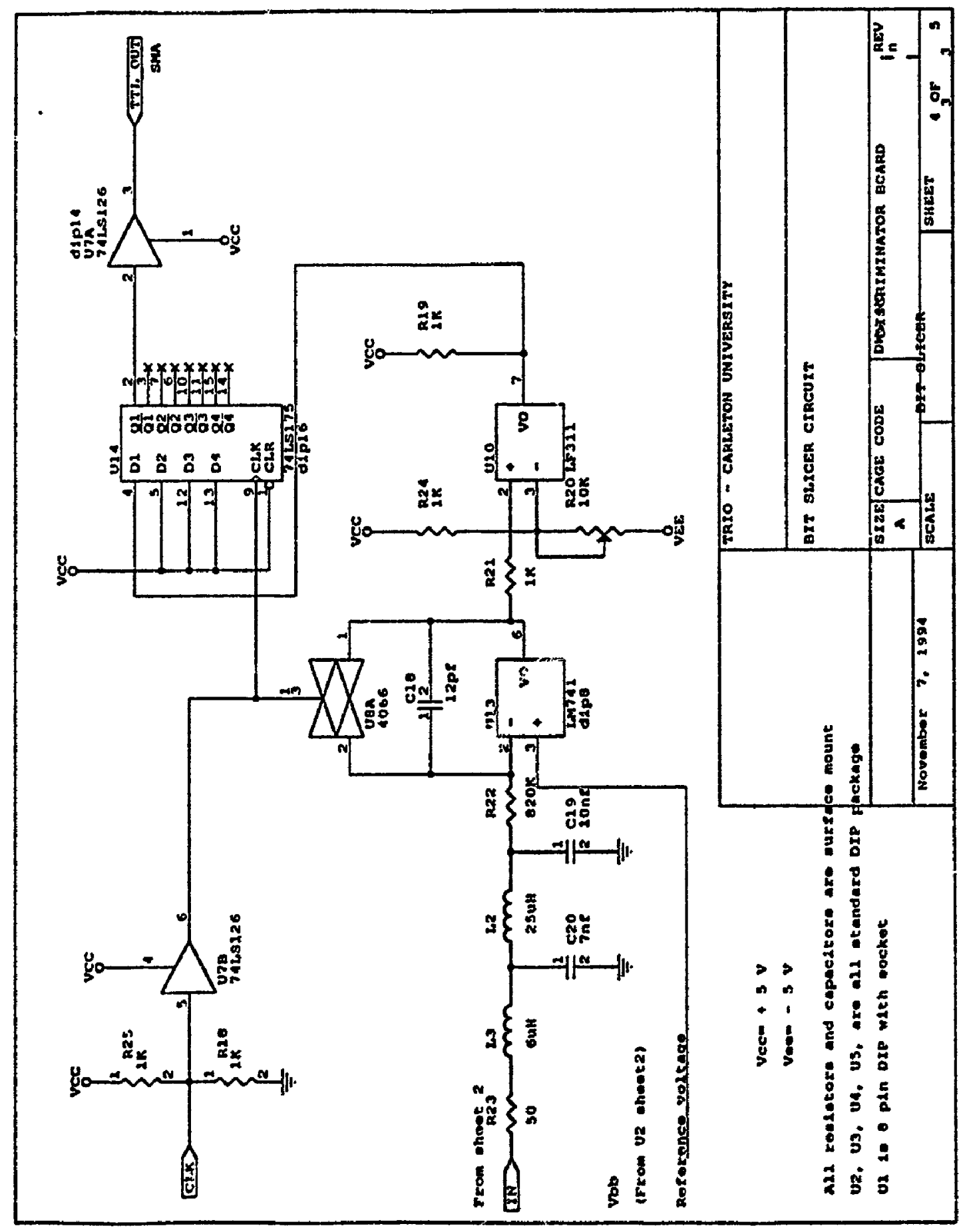

B.5 


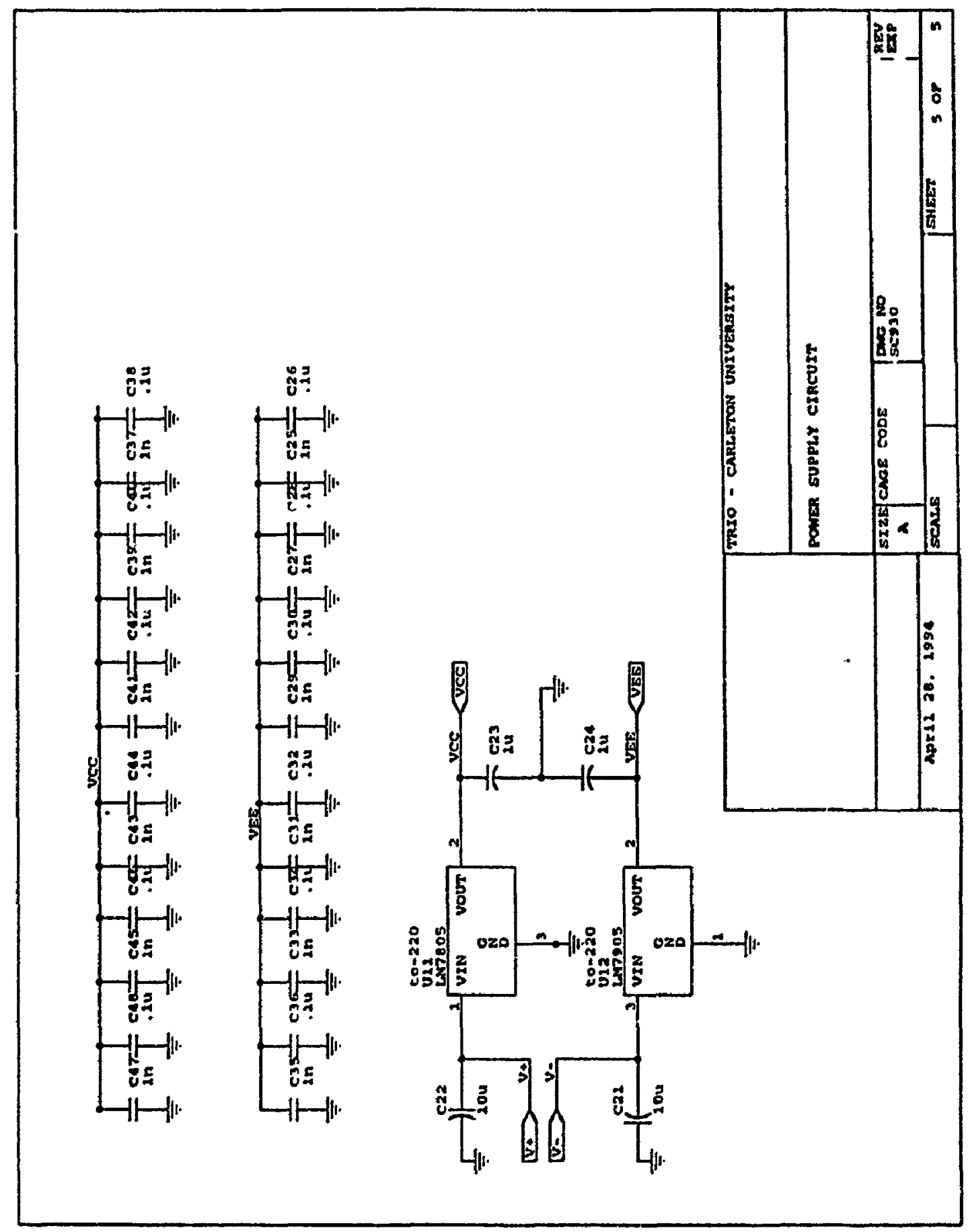

B.6 

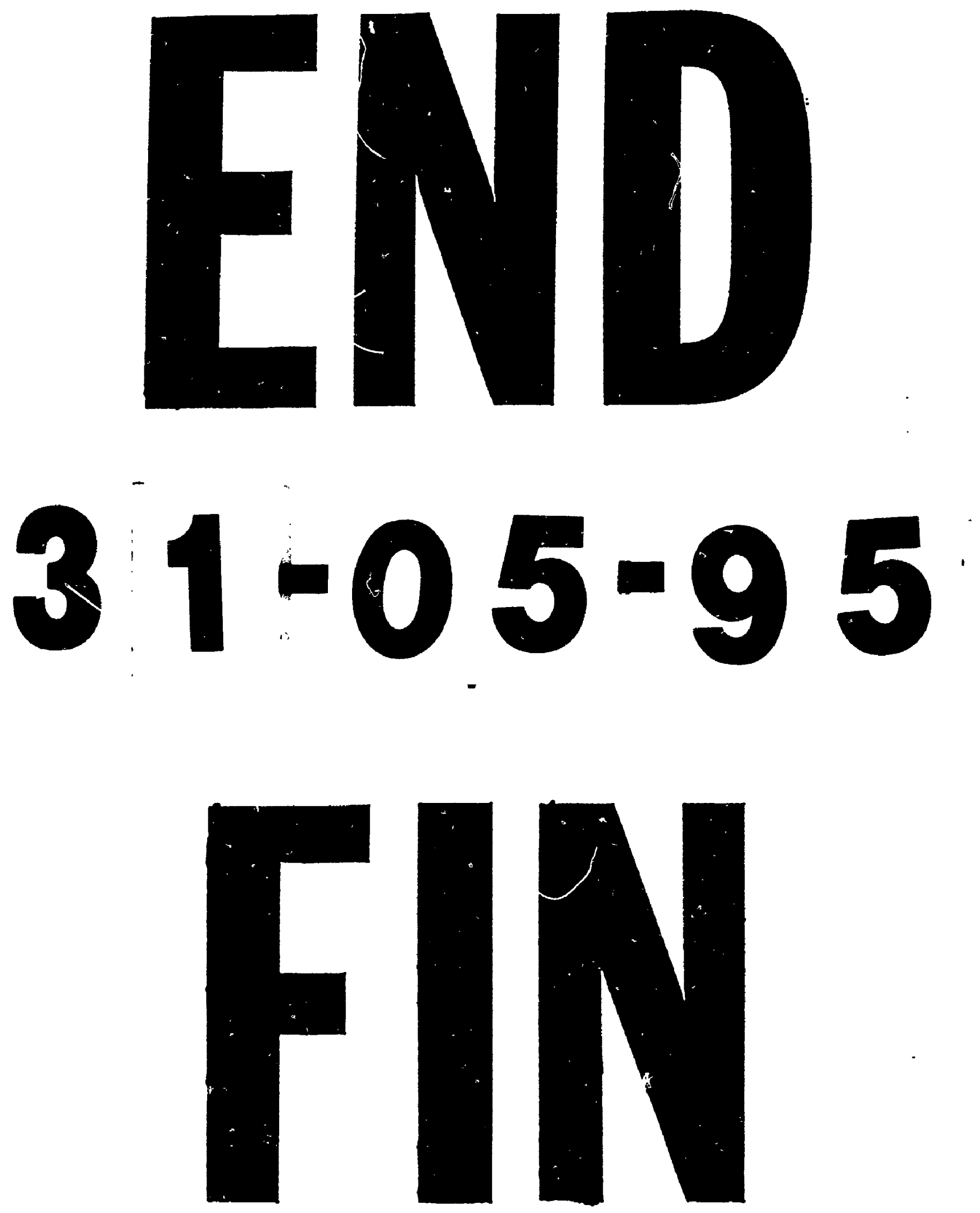